NTP RR 1

JULY 2016 


\title{
NTP Research Report on Systematic Literature Review on the Effects of Fluoride on Learning and Memory in Animal Studies
}

Research Report 1

July 2016

\author{
National Toxicology Program \\ Public Health Service \\ U.S. Department of Health and Human Services \\ ISSN: 2473-4756 \\ Research Triangle Park, North Carolina, USA
}




\section{Table of Contents}

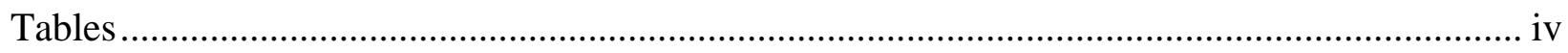

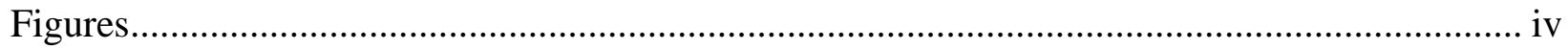

About This Report..............................................................................................................

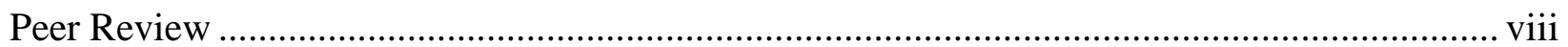

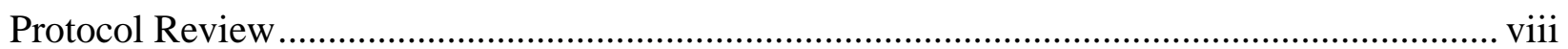

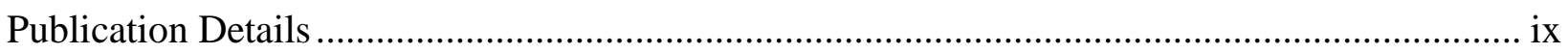

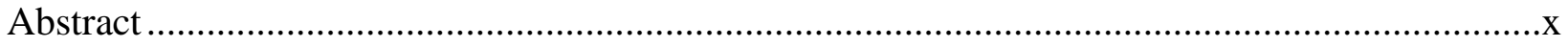

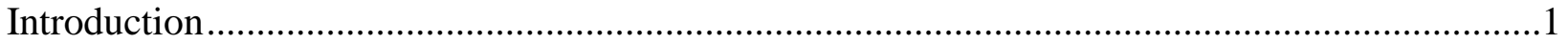

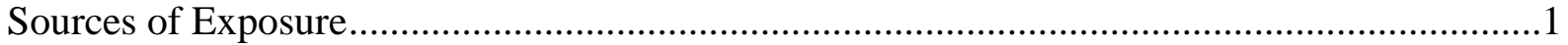

Use of Fluoride to Prevent Tooth Decay..............................................................................

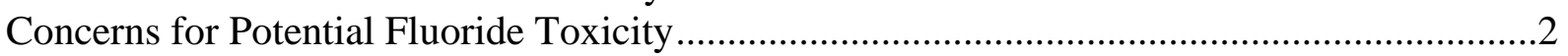

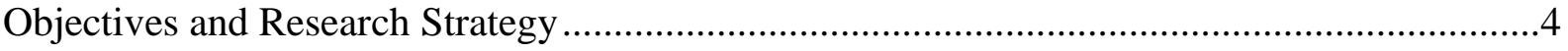

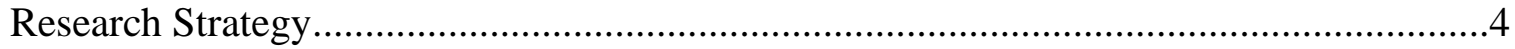

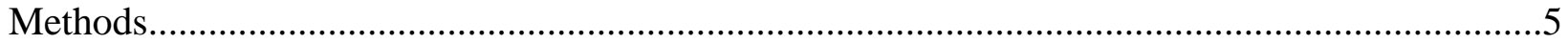

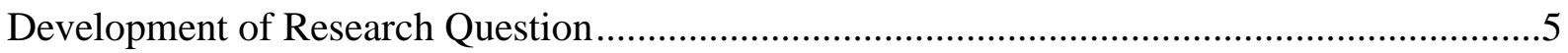

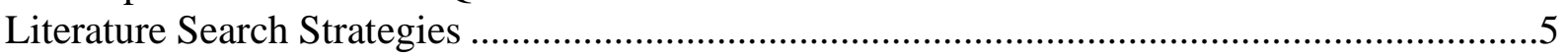

Searching Electronic Databases .................................................................................

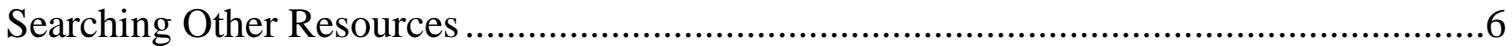

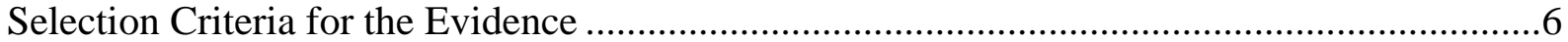

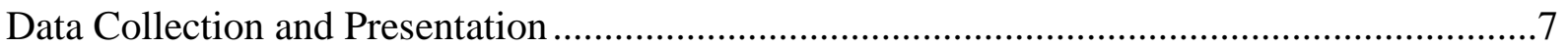

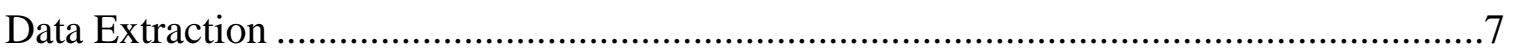

Quality Assessment of Individual Studies ...................................................................

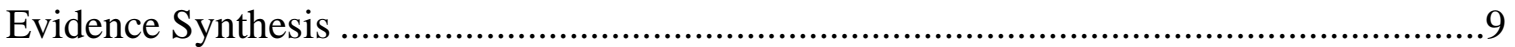

Assessment of Confidence in the Body of Evidence ......................................................10

Preparation of Level-of-evidence Conclusions................................................................13

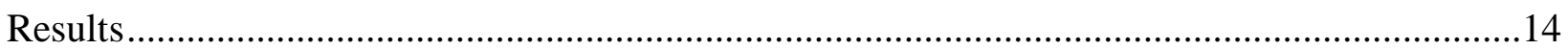

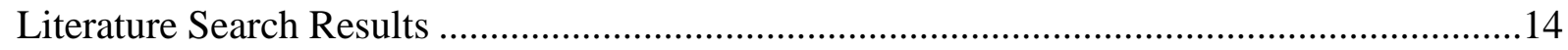

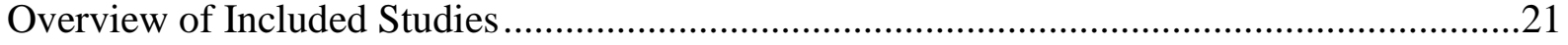

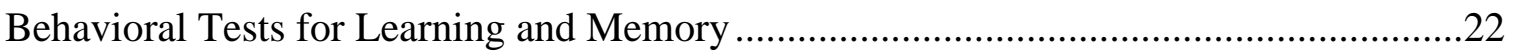

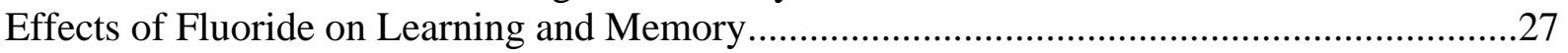

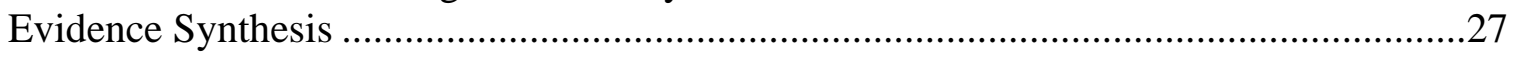

Exposure during Development ………………………………………………...27

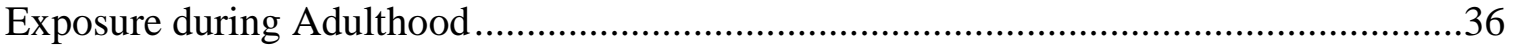

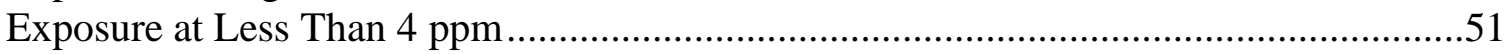

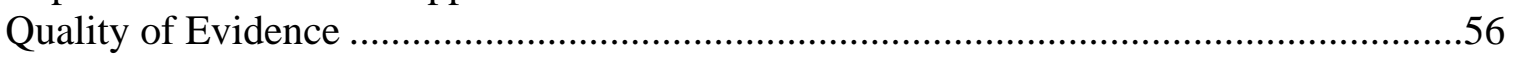

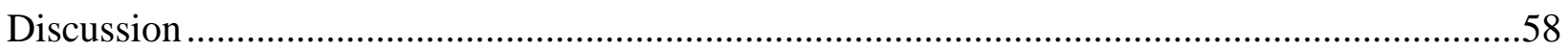

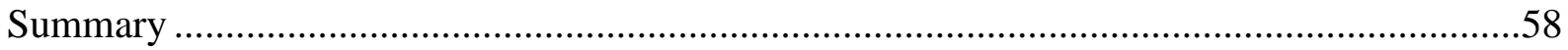

Relevance to Human Exposure Levels ............................................................................5

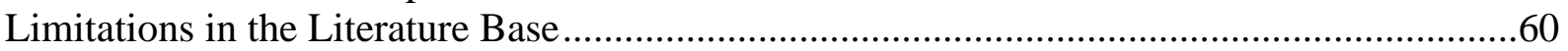

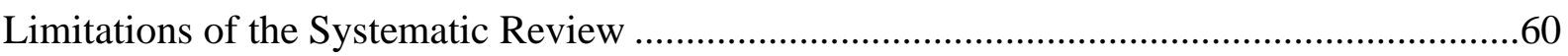




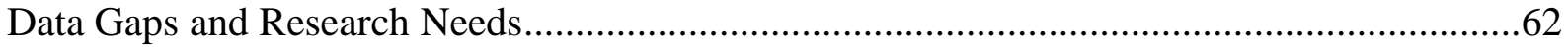

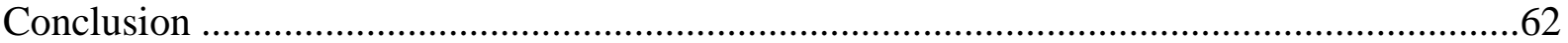

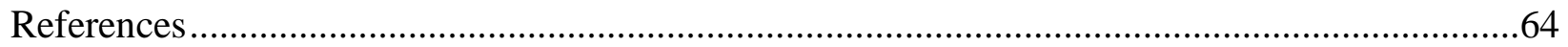

Appendix A. Electronic Database Search Strategies ...................................................... A-1

Appendix B. Data Extraction Elements for Animal Studies..................................................1

Appendix C. Studies Excluded from the Analysis for Having "Probably High" or "Definitely High" Risk of Bias in Multiple Critical Domains .............................. -1

Appendix D. Learning and Memory: Morris Water Maze (All Studies)................................ D-1

Appendix E. Learning and Memory: T-Maze...................................................................

Appendix F. Learning and Memory: Y-Maze .................................................................

Appendix G. Learning and Memory: Other Maze Tests ................................................ G-1

Appendix H. Learning and Memory: Exploratory Behavior ........................................... H-1

Appendix I. Learning and Memory: Passive Avoidance ................................................... I-1

Appendix J. Learning and Memory: Active Avoidance ........................................................

Appendix K. Motor and Sensory Function: Locomotor Activity .......................................... K-1

Appendix L. Motor and Sensory Function: Movement Coordination ......................................

Appendix M. Motor and Sensory Function: Reflex and Motor Sensory Development ...............1

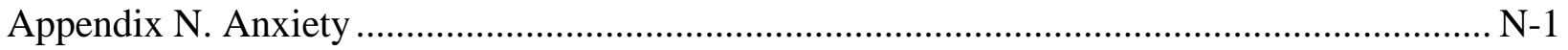

Appendix O. Grooming, Defecation, Urination............................................................... O-1

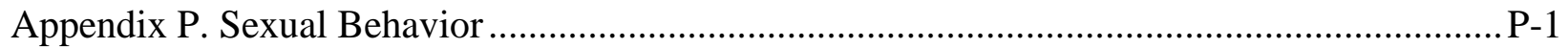

Appendix Q. Territorial Aggression ........................................................................ Q-1

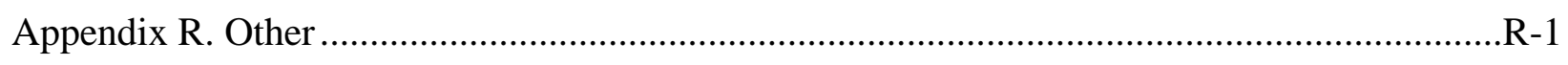

Appendix S. Fluoride Administered and Converted Doses (Individual Studies) ..................... S-1

Appendix T. Fluoride Administered and Converted Doses (Range across Studies for a

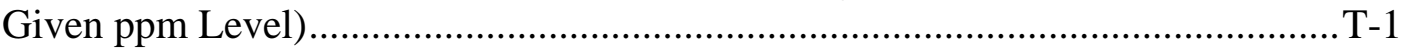

Appendix U. Pharmacokinetics and Concentrations of Fluoride Measured in Tissue, Blood, Urine, or Bone ................................................................................ U-1

Appendix V. Background Fluoride Levels in Drinking Water and Diet ............................... V-1 


\section{Tables}

Table 1. Representative Values for Fluoride Intakes Used in Calculation of the Relative Source Contribution from Drinking Water

Table 2. PECO (Populations, Exposures, Comparators, Outcomes) Statement ............................5

Table 3. OHAT Risk of Bias Questions for Evaluating Experimental Animal Studies ..................7

Table 4. Framework for Neurobehavioral Groupings............................................................9

Table 5. Key Factors When Considering Whether to Downgrade or Upgrade ...........................12

Table 6. Description of Relevant Studies ............................................................................21

Table 7. Learning and Memory Findings from Developmental Exposure Studies Excluded Based on Concern for High Overall Risk of Bias ........................................31

Table 8. Learning and Memory Findings from Adult Exposure Studies Excluded Based on Concern for High Overall Risk of Bias ...............................................................51

Table 9. Findings from Studies Testing <5 ppm Fluoride Excluded Based on Concern for

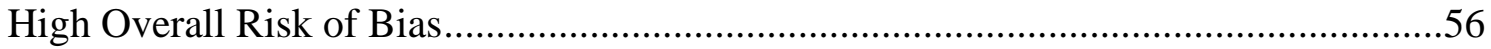

Table 10. Learning and Memory (Quality of Evidence and Summary of Findings) ....................57

\section{Figures}

Figure 1. Assessing Confidence in the Body of Evidence ("Quality of Evidence") ....................11

Figure 2. Translate Confidence Ratings into Evidence of Health Effect Conclusions from

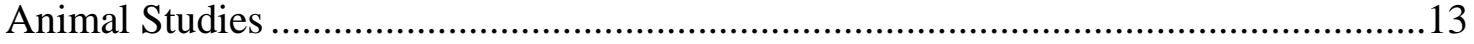

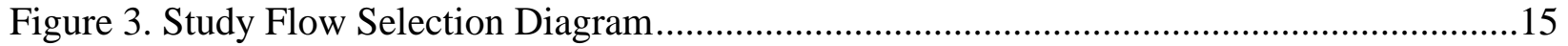

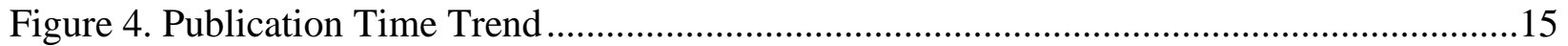

Figure 5. Results from Developmental Exposure Studies Using Morris Water Maze ..................33

Figure 6. Results from Developmental Exposure Studies in Other Maze, Exploration, and

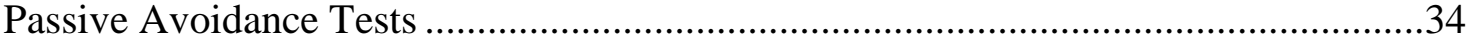

Figure 7. Risk of Bias in Studies in Studies Assessing Developmental Exposure to

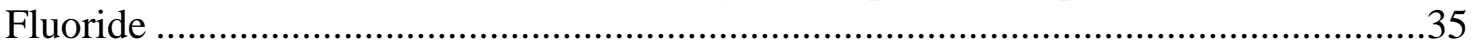

Figure 8. Results from Adult Exposure Studies Using Morris Water Maze ...............................39

Figure 9. Risk of Bias in Studies Assessing Adult Exposure to Fluoride Using the Morris

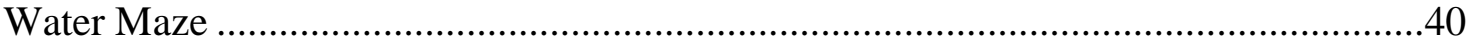

Figure 10. Results from Adult Exposure Studies Using Y- Maze and T-Maze ..........................43

Figure 11. Risk of Bias in Studies Assessing Adult Exposure to Fluoride Using the T- or Y-Maze.

Figure 12. Results from Adult Exposure Studies Using Active and Passive Avoidance Tests.

Figure 13. Risk of Bias in Adult Exposure Studies Using Active and Passive Avoidance Tests.

Figure 14. Results from Adult Exposure Studies Using Exploration and Operant Behavior Tests

Figure 15. Risk of Bias in Adult Exposure Studies Using Exploration and Operant

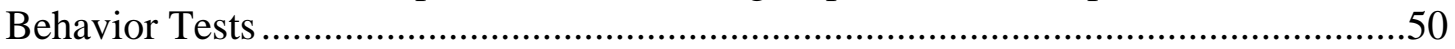

Figure 16. Results from All Studies Assessing Effects at $\leq 4$ ppm ........................................54

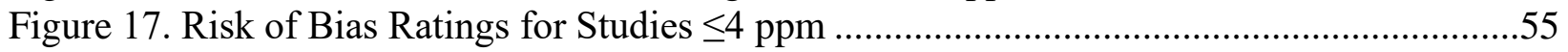

This report has been reformatted to meet new NTP publishing requirements; its content has not changed. 


\section{About This Report}

National Toxicology Program ${ }^{1}$

${ }^{1}$ Division of the National Toxicology Program, National Institute of Environmental Health

Sciences, Research Triangle Park, North Carolina, USA

\section{Collaborators}

Kristina Thayer, Jean Harry, Andrew Shapiro, Stephanie Holmgren, Mamta Behl, John Bucher, Greg Carter, Pamela Hartman, Cara Henning

Division of the National Toxicology Program, National Institute of Environmental Health Sciences, Research Triangle Park, North Carolina, USA

Contributed to conception or design and contributed to drafting of protocol

Jean Harry, Ph.D., Project Co-lead

Kristina Thayer, Ph.D., Project Co-lead

Mamta Behl, Ph.D.

John Bucher, Ph.D.

Andrew Shapiro, M.S.

Contributed to drafting of report

Jean Harry, Ph.D., Project Co-lead

Kristina Thayer, Ph.D., Project Co-lead

Critically reviewed draft report and figures

Mamta Behl, Ph.D.

John Bucher, Ph.D.

Stephanie Holmgren, M.S.

Andrew Shapiro, M.S.

Retrieved and managed references

Stephanie Holmgren, M.S.

Assessed risk of bias

Jean Harry, Ph.D.

Kristina Thayer, Ph.D.

ICF, Durham, North Carolina, USA

Critically reviewed draft report and figures

Pamela Hartman, M.E.M., Technical Lead

Greg Carter, M.E.M., Co-Lead Work Assignment Manager

Cara Henning, Ph.D., Co-Lead Work Assignment Manager

Extracted data

Pamela Hartman, M.E.M. 
Developed visualizations

Pamela Hartman, M.E.M.

\section{Contributors}

Division of the National Toxicology Program, National Institute of Environmental Health Sciences, Research Triangle Park, North Carolina, USA

Managed peer review of draft document and critically reviewed draft report and figures

Mary S. Wolfe, Ph.D.

ICF, Durham, North Carolina, USA

Coordinated peer review of draft document

Canden N. Byrd, B.S.

Assisted in document production

Penny Kellar, M.S.

Whitney Mitchell, B.S.

Managed article acquisition

Nicole Vetter, M.L.I.S.

Screened studies

Autumn Bordner, B.S.

Susan Goldhaber, M.P.H.

Maureen Malloy, B.A.

Extracted data

Susan Goldhaber, M.P.H.

Tao Hong, Ph.D.

Bryan Luukinen, M.S.P.H.

Johanna Rochester, Ph.D.

Pam Ross, M.P.H.

Assessed risk of bias

Robyn Blain, Ph.D.

Tao Hong, Ph.D.

University of Edinburgh, Department of Neurology and Translational Neuroscience, Coordinator of the Collaborative Approach to Meta-Analysis and Review of Animal Data in Experimental Studies, Edinburgh, Scotland

Technical advisor

Malcolm Macleod, Ph.D. 
Radboud University Medical Centre, Departments of SYstematic Review Centre for Laboratory Animal Experimentation (SYRCLE) and Anesthesiology, Nijmegen, Netherlands

Technical advisor

Carlijn Hooijmans, Ph.D. 


\section{Peer Review}

The research report, Systematic Literature Review on the Effects of Fluoride on Learning and Memory in Animal Studies, was evaluated by the reviewers listed below. These reviewers served as independent scientists, not as representatives of any institution, company, or governmental agency. In this capacity, reviewers determined if the design and conditions of these NTP studies were appropriate and ensured that this NTP Research Report presented the experimental results and conclusions fully and clearly.

\section{Peer Reviewers}

\section{Charles Vorhees, Ph.D.}

Division of Neurology

Cincinnati Children's Hospital Medical Center

Cincinnati, Ohio, USA

\section{Russell Carr, Ph.D.}

Center for Environmental Health Sciences

Department of Basic Sciences College of Veterinary Medicine

Mississippi State University

Starkville, Mississippi, USA

\section{Protocol Review}

The protocol and design were evaluated by the reviewers listed below.

\section{Emily Sena, Ph.D.}

Coordinator of the Collaborative Approach to Meta-Analysis and Review of Animal Data in Experimental Studies

Department of Neurology and Translational Neuroscience

University of Edinburgh

Edinburgh, Scotland

\section{Staff}

National Industrial Chemicals Notification and Assessment Scheme

Department of Health

Australian Government

Canberra, Australia 


\section{Publication Details}

Publisher: National Toxicology Program

Publishing Location: Research Triangle Park, North Carolina

ISSN: 2473-4756

DOI: https://doi.org/10.22427/NTP-RR-1

Report Series: NTP Research Report Series

Report Series Number: 1

Official citation: National Toxicology Program (NTP). 2016. NTP research report on systematic literature review on the effects of fluoride on learning and memory in animal studies. Research Triangle Park, NC: National Toxicology Program. Research Report 1. 


\section{Abstract}

Background: Previous systematic reviews of epidemiology studies have found support for a geographical association between high levels of naturally occurring fluoride in water $(>1.5 \mathrm{ppm})$ and lower IQ in children. Most of the evidence from humans is from fluoride-endemic regions having higher background levels of fluoride compared to the fluoride concentrations historically used in community water fluoridation programs (0.7-1.2 ppm). Confidence in this body of evidence is limited, primarily due to poor reporting quality, lack of consideration of confounding (e.g., nutritional status, socioeconomic status, iodine deficiency), and concern for co-exposures to relatively high levels of other known neurotoxicants such as lead or arsenic. A systematic review of experimental animal studies could help in interpreting the human evidence.

Objective: To investigate whether fluoride exposure has detrimental impacts on neurobehavior in laboratory animal studies, prioritizing assessment of learning and memory outcomes. Confidence in the body of evidence was assessed according to one of four statements: (1) High, (2) Moderate, (3) Low, or (4) Very Low/No Evidence Available.

Methods: We included experimental animal studies that used mammalian species (whole organism) exposed during development or adulthood, which compared the effects of oral exposure to various fluoride concentrations to vehicle controls on neurobehavioral responses. The principal outcomes were learning and memory, but other neurobehavioral studies were included (e.g., anxiety, motor activity, aggression, sexual behavior). Studies assessing brainrelated cellular, morphometric or histological endpoints were considered beyond the scope of this analysis. A literature search was performed up to January 14, 2016, using PubMed, BIOSIS, EMBASE, Scopus, Web of Science, PsycINFO, and several specialized databases. There were no date or language restrictions, and unpublished data and abstracts were excluded. Risk of bias was assessed regarding randomization, allocation concealment, blinding, exposure characterization, health outcome assessment, incomplete outcome data, selective outcome reporting, and other biases.

Results: The database searches yielded 4,643 unique records and 13 records were identified from other sources. Of the 4,656 studies, we identified 68 studies using mice or rats and testing drinking water or dietary concentrations of 0.45 to $272 \mathrm{ppm}$ fluoride $(0.12$ to $40 \mathrm{mg} / \mathrm{kg}-\mathrm{d})$. Most included studies were published after 2000. Forty-eight studies addressed learning and memory, 16 of which assessed exposure during development.

Synthesis of results: Meta-analysis was not conducted due to the small number of studies that measured endpoints similarly based on study design, that is, dose levels, duration of treatment, lifestage at exposure, species, or differences in measurement of behavioral responses. Relatively few studies provided information on other sources of fluoride (e.g., diet, water source). Most studies were statistically underpowered to detect a $<20 \%$ change from control groups for behavioral tests. Approximately $30 \%$ of the learning and memory studies were considered to have a very serious risk of bias and were excluded from the narrative analysis. Conclusions were reached based on an analysis of 32 studies. Results show low-to-moderate confidence for a pattern of findings suggestive of an effect on learning and memory based on developmental and adult exposure studies. The evidence is strongest (moderate level-of-evidence) in animals exposed as adults and weaker (low level-of-evidence) in animals exposed during development. Level-of-evidence conclusions were rated down due to concern for indirectness and risk of bias. The evidence was strongest and most abundant for adult exposure studies using the Morris water 
maze. In many cases, across the entire dose range tested, whether the effects were specifically related to learning and memory - versus a possible impact on motor or sensory function that could have impaired the ability of the animal to perform the learning and memory tests as measured-was not possible to discern. This was considered a form of indirectness. Additional studies are required to have higher confidence in the specificity of the responses as learning or memory impairments and in quantitative measures such as the effect sizes, point of departure, identification of no observed effect level or lowest observed effect level doses, or parameters for benchmark dose analysis. Based on control values (means and standard deviations/standard errors) and the number of animals per group, the studies appear statistically underpowered to detect a $<10 \%$ or $<20 \%$ change from controls for most behavioral endpoints.

Conclusion: Very few studies assessed learning and memory effects in experimental animals (rats and mice) at exposure levels near 0.7 parts per million, the recommended level for community water fluoridation in the United States. At concentrations higher than 0.7 parts per million, this systematic review found a low to moderate level-of-evidence that suggests adverse effects on learning and memory in animal exposed to fluoride. The evidence is strongest (moderate level-of-evidence) in animals exposed as adults and weaker (low level-of-evidence) in animals exposed during development. Confidence in these findings was reduced primarily based on potential confounding of the learning and memory assessments by deficits in motor function or fear and risk of bias limitations. Additional research is needed, in particular to address potential effects on learning and memory following exposure during development to fluoride at levels nearer to 0.7 parts per million. NTP is conducting laboratory studies in rodents to fill data gaps identified by this systematic review of the animal studies. The findings from those studies will be included in a future systematic review to evaluate potential neurobehavioral effects from exposure to fluoride during development with consideration of human, experimental animal and mechanistic data. 


\section{Introduction}

\section{Sources of Exposure}

In 2010, the US Environmental Protection Agency (EPA) conducted a relative source contribution analysis of fluoride. Sources include drinking water, foods, beverages, dental products (toothpaste, mouth rinses), supplements, industrial emissions, pharmaceuticals, and pesticides (e.g., cryolite, sulfuryl fluoride). Soil ingestion is another source of exposure in young children (US EPA 2010b). The major contributors to exposure are drinking water, beverages, food, and toothpaste. The relative source contribution from drinking water intake was 40-60\% after the age of 1 year and $70 \%$ in children less than 1 year old.

Table 1. Representative Values for Fluoride Intakes Used in Calculation of the Relative Source Contribution from Drinking Water

\begin{tabular}{ccccccccc}
\hline $\begin{array}{c}\text { Age Group } \\
(\mathbf{y e a r s})\end{array}$ & $\begin{array}{c}\mathbf{D W I}^{\mathbf{a}} \\
(\mathbf{m g} / \mathbf{d})\end{array}$ & $\begin{array}{c}\text { BI } \\
(\mathbf{m g} / \mathbf{d})\end{array}$ & $\begin{array}{c}\text { FI } \\
(\mathbf{m g} / \mathbf{d})\end{array}$ & $\begin{array}{c}\text { TI } \\
(\mathbf{m g} / \mathbf{d})\end{array}$ & $\begin{array}{c}\text { SuF } \\
(\mathbf{m g} / \mathbf{d})\end{array}$ & $\begin{array}{c}\text { SI } \\
(\mathbf{m g} / \mathbf{d})\end{array}$ & Total (mg/d) & RSC (\%) \\
\hline $0.5-<1$ & 0.84 & - & $0.25^{\mathrm{b}}$ & 0.07 & 0.03 & 0.02 & 1.2 & 70 \\
$1-<4$ & 0.63 & 0.36 & 0.16 & 0.34 & 0.05 & 0.04 & 1.58 & 40 \\
$4-<7$ & 0.82 & 0.54 & 0.35 & 0.22 & 0.06 & 0.04 & 2.03 & 40 \\
$7-<11$ & 0.86 & 0.60 & 0.41 & 0.18 & 0.07 & 0.04 & 2.16 & 40 \\
$11-14$ & 1.23 & 0.38 & 0.47 & 0.20 & 0.09 & 0.04 & 2.41 & 51 \\
$>14$ & $1.74^{\mathrm{b}}$ & 0.59 & 0.38 & $0.10^{\mathrm{c}}$ & 0.08 & 0.02 & 2.91 & 60 \\
\hline
\end{tabular}

From Table 7-2 (US EPA 2010b).

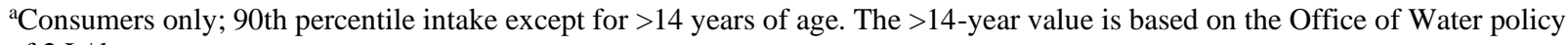
of $2 \mathrm{~L} / \mathrm{d}$.

bincludes foods, fluoride in powdered formula, and fruit juices; no allocation for other beverages.

${ }^{\mathrm{c}}$ Assumed. $50 \%$ of the 11- to 14-year-old age group. DWI = drinking water intake; BI = beverage intake; FI = food intake (solid foods); $\mathrm{TI}=$ toothpaste intake; $\mathrm{SuF}=$ sulfuryl fluoride intake; $\mathrm{SI}=$ soil intake; RSC = relative source contribution.

EPA has proposed a reference dose (RfD) of $0.08 \mathrm{mg} / \mathrm{kg}-\mathrm{d}$ for protection against pitting of tooth enamel (severe dental fluorosis), and this value also is considered protective against fractures and skeletal effects in adults (US EPA 2010a).

\section{Use of Fluoride to Prevent Tooth Decay}

Fluoride from community water fluoridation, mouth rinses, gels, and toothpastes is intended to prevent dental caries primarily through topical remineralization of tooth surfaces. Community water fluoridation and fluoride toothpaste are the most common sources of non-dietary fluoride in the United States (US DHHS 2015). Because fluorine is the 13th most abundant element in Earth's crust, fluoride also naturally occurs in water and is present in many non-fluoridated water systems.

Although other fluoride-containing products and sources are available (e.g., mouth rinses, dietary supplements, professionally applied fluoride compounds), community water fluoridation has been identified as the most cost-effective method for delivering fluoride to all members of the community regardless of age, educational attainment, or income level. Consuming fluoridated water and beverages and foods prepared or processed with fluoridated water throughout the day maintains a low concentration of fluoride in saliva and plaque, which enhances remineralization 
(US DHHS 2015). Community water fluoridation to minimize the occurrence and severity of tooth decay, which began in 1945, had reached $67 \%$ of the US population by 2012 . About 25 countries practice community water fluoridation (Iheozor-Ejiofor et al. 2015), and many more provide fluoride through other means such as salt. In 2012, an estimated 200 million people in the United States were served by 12,341 community water systems that added fluoride to water or purchased water with added fluoride from other systems (US DHHS 2015).

The US Public Health Service (PHS) first recommended communities add fluoride to drinking water in 1962. PHS guidance is advisory, not regulatory, which means that although PHS recommends community water fluoridation as an effective public health intervention, state and local governments decide whether to fluoridate water systems. For community water systems that add fluoride, PHS currently recommends a fluoride concentration of 0.7 milligrams/liter (mg/L, ppm). ${ }^{1}$ This recommended level provides the best balance of protection from dental caries, while limiting the risk of dental fluorosis. Dental fluorosis is a condition marked by changes in the appearance of tooth enamel most commonly appearing as lacy white markings (US DHHS 2015). Dental fluorosis can result when children regularly consume fluoride from birth through 8 years of age - the time when their permanent teeth (with the exception of the third molars) are developing.

Under the Safe Drinking Water Act, EPA sets standards for drinking water quality. Currently, the enforceable fluoride standard is set at $4.0 \mathrm{mg} / \mathrm{L}$ to protect consumers from exposure to drinking water sources with naturally high occurrence of fluoride against severe skeletal fluorosis (a condition caused by excessive fluoride intake over a long period that, in advanced stages, can cause pain, crippling damage to bones and joints, or both). EPA also has a secondary drinking water standard of $2.0 \mathrm{mg} / \mathrm{L}$ to protect against moderate to severe dental fluorosis. This secondary standard is not enforceable but requires water systems to notify the public. EPA is currently reviewing the drinking water standards for fluoride (US EPA 2013).

\section{Concerns for Potential Fluoride Toxicity}

The most commonly cited health concerns about fluoride and water fluoridation focus on bone fractures and skeletal fluorosis, intelligence quotient (IQ) and other neurological effects, cancer, and endocrine disruption. Effects on neurological function, endocrine functions (thyroid, parathyroid, pineal), metabolism (glucose), and carcinogenicity were assessed in the 2006 National Research Council (NRC) report, Fluoride in Drinking Water: A Scientific Review of EPA's Standards (NRC 2006). The review considered adverse effects of water fluoride, focusing on concentrations of $2-4 \mathrm{mg} / \mathrm{L},{ }^{2}$ a range higher than the current recommendation for community water fluoridation $(0.7 \mathrm{mg} / \mathrm{L})$. At levels below $4.0 \mathrm{mg} / \mathrm{L}, \mathrm{NRC}$ found no evidence substantial enough to support negative health effects other than severe dental fluorosis. The conclusions from the NRC review were the primary source of information for the potential hazard summary in a 2015 report by the US Department of Health and Human Services (DHHS), Federal Panel on Community Water Fluoridation. The NRC report noted several challenges to evaluating the

${ }^{1}$ For many years, most fluoridated community water systems used fluoride concentrations ranging from 0.8 to $1.2 \mathrm{mg} / \mathrm{L}$ (US DHHS 2015).

${ }^{2}$ EPA's maximum contaminant level goal (MCLG) for fluoride is $4 \mathrm{mg} / \mathrm{L}$ and secondary maximum contaminant level (SMCL) is $2 \mathrm{mg} / \mathrm{L}$. MCLGs establish an exposure guideline to prevent adverse health effects in the general population, and SMCLs are intended to reduce the occurrence of adverse cosmetic consequences from exposure to fluoride. Both the MCLG and the SMCL are nonenforceable guidelines (NRC 2006). 
literature, including deficiencies in reporting quality; consideration of all sources of fluoride exposure; consideration of potential confounding; selection of appropriate control subject populations in epidemiology studies; demonstrated clinical significance of endocrine effects; and the biological relationship between histological, biochemical, and molecular alterations with behavioral effects. Regarding neurotoxicity and neurobehavioral effects, the main conclusions in the 2006 NRC report are:

"Animal and human studies of fluoride have been published reporting adverse cognitive and behavioral effects. A few epidemiologic studies of Chinese populations have reported IQ deficits in children exposed to fluoride at 2.5 to $4 \mathrm{mg} / \mathrm{L}$ in drinking water. Although the studies lacked sufficient detail for the committee to fully assess their quality and relevance to U.S. populations, the consistency of the results appears significant enough to warrant additional research on the effects of fluoride on intelligence." [p. 8] (NRC 2006)

"A few animal studies have reported alterations in the behavior of rodents after treatment with fluoride, but the committee did not find the changes to be substantial in magnitude. More compelling were studies on molecular, cellular, and anatomical changes in the nervous system found after fluoride exposure, suggesting that functional changes could occur. These changes might be subtle or seen only under certain physiological or environmental conditions. More research is needed to clarify the effect of fluoride on brain chemistry and function." [p. 8] (NRC 2006)

Since release of the 2006 NRC report, approximately 10 epidemiological studies of children's IQ have been published. A 2015 systematic analysis of the human literature conducted for the Republic of Ireland's Department of Health (Sutton et al. 2015) reviewed the new literature and concluded no evidence of an association with lowered IQ was apparent in studies of community water fluoridation. The authors based this conclusion primarily on an analysis of a prospective cohort study conducted in New Zealand (Broadbent et al. 2015). For fluoride-endemic areas, there was a strong suggestion that high levels of naturally occurring fluoride in water $(>1.5 \mathrm{ppm})$ could be associated with negative health effects, including lowering of IQ. Overall, these studies were considered low quality, as they did not fully account for known confounding factors with regard to IQ (e.g., nutritional status, socioeconomic status), nor other potential influencing factors (e.g., iodine deficiency, chemical contaminants in the ground water such as arsenic and lead). The conclusions of Sutton et al. (2015) are consistent with findings of a 2012 metaanalysis of 27 epidemiology studies that supported the possibility of an adverse effect of "high" fluoride exposure ${ }^{3}$ on children's neurodevelopment, specifically for lowered IQ (Choi et al. 2012). The Choi et al. meta-analysis, however, also identified study quality limitations, primarily related to reporting quality, that limited the strength of the conclusions (Choi et al. 2012). More than 35 experimental animal studies evaluating the neurobehavioral effects of fluoride have been published since release of the 2006 NRC report. A systematic review of experimental studies that focuses on the effects of fluoride on learning and memory would help interpret the human literature on IQ and identify key research/data gaps for additional study.

For cancer and endocrine disruption, the National Toxicology Program (NTP) is analyzing the amount of evidence available and the merit of pursuing systematic reviews given factors such as

\footnotetext{
3"High" was defined based on drinking water concentration, evidence of fluorosis, exposure related to coal-burning activities, and urine levels.
} 
the extent of new research published since previous evaluations and whether these new reports address or correct the deficiencies noted in the literature (OEHHA 2011; NRC 2006; SCHER 2011).

\section{Objectives and Research Strategy}

The overall objective of this evaluation is to undertake a systematic review of the existing animal studies to develop NTP level-of-evidence conclusions (NTP 2015a) about whether fluoride exposure is associated with impairments in learning and memory.

\section{Research Strategy}

- Identify literature reporting the effects of exposure to fluoride and neurobehavioral outcomes in experimental animal studies utilizing mammalian species (whole organism).

- Extract data on neurobehavioral outcomes from identified studies. Primary outcomes are learning and memory, but other behavioral measures are summarized (e.g., "anxiety," motor function).

- Assess the internal validity ("risk of bias") of individual studies.

- Summarize the extent of evidence available.

- Synthesize the evidence, and perform quantitative meta-analyses if appropriate, and evaluate sources of heterogeneity.

- Rate confidence in the body of evidence for effects on learning and memory according to one of four statements: (1) High, (2) Moderate, (3) Low, or (4) Very Low/No Evidence Available.

- Translate confidence ratings into level-of-evidence for effects on learning and memory according to one of four statements: (1) High, (2) Moderate, (3) Low, or (4) Inadequate.

- Describe limitations of the evidence base, limitations of the systematic review, and findings in the context of human exposure levels.

- Identify data gaps and key research needs. 
Systematic Literature Review on the Effects of Fluoride on Learning and Memory in Animal Studies

\section{Methods}

The systematic review was conducted based on guidance outlined in the Office of Health Assessment and Translation (OHAT) Handbook for Conducting a Literature-Based Health Assessment (NTP 2015a). Methods are summarized below and presented in more detail in the study protocol available at https://hawcproject.org/assessment/126/.

\section{Development of Research Question}

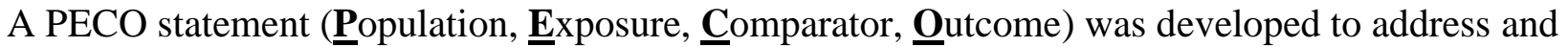
understand potential effects of fluoride on neurobehavioral measures in animal models, especially related to learning and memory (Table 2).

Table 2. PECO (Populations, Exposures, Comparators, Outcomes) Statement

\begin{tabular}{|c|c|}
\hline PECO & Evidence \\
\hline Exposure & $\begin{array}{l}\text { Fluoride at any concentration; relevant forms are those used as additives for water fluoridation: } \\
\text { fluorosilicic acid (also called hydrofluorosilicate; CASRN 16961-83-4) } \\
\text { sodium hexafluorosilicate (also called disodium hexafluorosilicate or sodium fluorosilicate; 16893-85-9) } \\
\text { sodium fluoride (CASRN 7681-49-4) } \\
\text { other forms that readily dissociate into free fluoride ions (e.g., potassium fluoride, calcium fluoride, } \\
\text { ammonium fluoride) }\end{array}$ \\
\hline Comparator & Animals exposed to vehicle-only treatment \\
\hline Outcomes & $\begin{array}{l}\text { Any neurobehavioral outcome } \\
\text { Primary outcomes: learning and memory } \\
\text { Secondary outcomes: motor and sensory function, and other aspects of behavior that do not fall under } \\
\text { learning and memory (e.g., anxiety, territorial aggression) }\end{array}$ \\
\hline
\end{tabular}

\section{Literature Search Strategies}

\section{Searching Electronic Databases}

Systematic search strategies were developed to identify all relevant published evidence on the health effects of water fluoridation by using index terms and text words based on key elements of the research question. Nine electronic databases ${ }^{4}$ initially were searched on January 7, 2015, and the search was updated on January 14, 2016, unless noted otherwise:

- BIOSIS (Thomson Reuters - searched 2/18/2015)

- EMBASE (Elsevier)

- European Chemicals Agency (ECHA) Registration dossiers ("REACH") database (searched 3/24-27/2015)

\footnotetext{
${ }^{4}$ NLM's Toxline database was not included in the search because recent changes have resulted in significant reductions in search functionality that limits running the search strings for this topic. In addition, the other databases are very likely to identify relevant published and peer-reviewed animal studies.
} 
- Organization for Economic Co-operation and Development (OECD) Existing Chemicals Screening Information Data Sets (SIDS) database (searched 2/18/2015)

- PsycINFO (APA [American Psychological Association] PsycNet)

- PubMed (National Library of Medicine [NLM])

- Scopus (Elsevier)

- USEPA HPV Challenge Program Robust Summaries and Test Plans database ([High Production Volume] searched 3/23/2015)

- Web of Science (Thomson Reuters; Web of Science indexes the journal, Fluoride)

No publication date or language restrictions were applied. Full details of the search strategy for each database are presented in Appendix A.

\section{Searching Other Resources}

The reference lists of included studies, records that do not contain original data (i.e., reviews, editorials, or commentaries), and the Fluoride Action Network website ("Fluoride affects learning and memory in animals" at http://fluoridealert.org/studies/brain02/) were searched for additional relevant publications.

\section{Selection Criteria for the Evidence}

Studies were screened for inclusion using a structured form in DistillerSR (Evidence Partners; https://distillercer.com/products/distillersr-systematic-review-software/). Two members of the evaluation design team independently conducted a title and abstract screen of the search results to identify studies that met the eligibility criteria. Studies that were not excluded based on the title and abstract advanced to full-text review. For citations with no abstract, articles were screened based on all or some of the following: title relevance (title should indicate clear relevance), page numbers (articles two pages in length or less were assumed conference reports, editorials, or letters), and PubMed MeSH (Medical Subject Headings).

Full-text copies of potentially relevant articles were independently assessed by two reviewers to identify studies that satisfied the inclusion and exclusion criteria. Discrepant screening results were resolved by discussion. Assessment of eligibility status of non-English studies was facilitated by native-language speakers at ICF International or National Institute of Environmental Health Sciences (NIEHS).

To be eligible for inclusion, studies had to comply with the criteria specified by the PECO statement (Table 2). Studies that did not meet the PECO criteria were excluded. In addition, the following exclusion criteria were applied:

- Studies did not contain original data, such as reviews, editorials, or commentaries.

- Studies were not peer reviewed (e.g., conference abstracts, technical reports, theses/dissertations, working papers from research groups or committees, white papers). 
Systematic Literature Review on the Effects of Fluoride on Learning and Memory in Animal Studies

\section{Data Collection and Presentation}

\section{Data Extraction}

Data were extracted from individual studies by members of the evaluation team using DRAGON (Dose Response Analytical Generator and Organizational Network) and Health Assessment Workspace Collaborative (HAWC) ${ }^{5}$ software. Data extraction elements collected from animal studies are listed in Appendix B Information on concentrations of fluoride measured in brain, blood, urine, or bone also was summarized when available. One member of the evaluation team extracted the data, which a second member checked. Missing data from individual studies were not sought. Extracted data were warehoused using HAWC.

\section{Quality Assessment of Individual Studies}

Risk of bias ratings for individual studies were assessed using the OHAT tool (NTP 2015b) (Table 3). Two raters assessed the studies, answering nine risk of bias questions following guidance outlined in OHAT's risk of bias documentation (NTP 2015b). After one reviewer independently determined risk of bias across all questions, a second person went through and agreed or disagreed with the ratings. Differences in ratings were discussed and resolved. Authors were queried to obtain missing information, and responses received were used to evaluate risk of bias. Information that was not reported was assumed not conducted (e.g., randomization, blinding), resulting in an assessment of "probably high" risk of bias.

Table 3. OHAT Risk of Bias Questions for Evaluating Experimental Animal Studies

OHAT Risk of Bias Questions for Evaluating Experimental Animal Studies

\section{Questions}

\section{Selection Bias}

1. Was administered dose or exposure level adequately randomized?

2. Was allocation to study groups adequately concealed?

\section{Performance Bias}

3. Were experimental conditions identical across study groups?

4. Were the research personnel and human subjects blinded to the study group during the study?

\section{Attrition/Exclusion Bias}

5. Were outcome data complete with respect to attrition or exclusion from analysis?

\section{Detection Bias}

6. Can we be confident in the exposure characterization?

7. Can we be confident in the outcome assessment?

\section{Selective Reporting Bias}

8. Were all measured outcomes reported?

\section{Other Sources of Bias}

9. Were there no other potential threats to internal validity (e.g., statistical methods were appropriate)?

\footnotetext{
${ }^{5}$ ICF International. 2014. From Systematic Review to Assessment Development: Managing Big (and Small) Datasets with DRAGON. http://www.icfi.com/insights/products-and-tools/dragon-online-tool-systematic-review. Health Assessment Workspace Collaborative (HAWC): A Modular Web-based Interface to Facilitate Development of Human Health Assessments of Chemicals. https://hawcproject.org/portal/.
} 
Systematic Literature Review on the Effects of Fluoride on Learning and Memory in Animal Studies

OHAT Risk of Bias Questions for Evaluating Experimental Animal Studies

\section{Response Options}

Definitely Low risk of bias:

There is direct evidence of low risk of bias practices.

Probably Low risk of bias:

There is indirect evidence of low risk of bias practices OR it is deemed that deviations from low risk of bias practices for these criteria during the study would not appreciably bias results.

Probably High risk of bias:

There is indirect evidence of high risk of bias practices OR there is insufficient information (e.g., not reported or "NR") provided about relevant risk of bias practices.

Definitely High risk of bias:

There is direct evidence of high risk of bias practices.

\section{Critical Risk of Bias Domains}

Randomization to treatment group, blinding during neurobehavioral outcome assessment, adequate characterization of the administered chemical, and controlling for litter effects in developmental studies were considered key factors in determining whether a study had an overall very serious risk of bias concern, referred to as a "tier 3" study in the OHAT Handbook (NTP 2015a). Studies considered as having "probably high" or "definitely high" risk of bias in several of these domains were classified as tier 3 studies and excluded from the main analysis, although the impact of excluding the studies was considered. Studies also may be considered tier 3 due to a combination of concern for risk of bias in a critical domain(s) and very poor reporting quality (e.g., not reporting the number of animals treated).

Randomization and blinding during outcome assessment were considered especially critical factors for risk of bias assessment because of empirical support that failure to implement these factors can bias results away from the null toward larger effects (Higgins and Green 2011; Krauth et al. 2013). The extent of empirical support documenting these biases in animal studies is better documented compared to other risk of bias factors, for example, blinding during allocation to study groups or during the course of the study (NTP 2015b). In addition, concern for lack of blinding during allocation or the conduct of the study can be attenuated if blinding was implemented at outcome assessment. For these reasons, blinding at outcome assessment was weighed more heavily during risk of bias assessment than blinding during allocation concealment or during the course of the study. In neurobehavioral studies, concern for lack of blinding at outcome assessment is attenuated if behavioral parameters are measured by an automated, computer-driven system.

Adequate characterization of the test compound is necessary to evaluate the purity and stability of the chemical exposure. Independent verification of purity would be considered best practice because the identity and purity as listed on the bottle can be inaccurate. In NTP's experience, about $3 \%$ of chemicals purchased are the wrong chemical, and the inaccuracy rate of chemical labeling increases to $10 \%$ if inaccurate reporting of purity is included (unpublished, personal communication Brad Collins, NTP chemist). Impurities also might be more toxic than the compound of interest.

Adequate control for litter effects when littermates are used in an experiment is considered essential in developmental studies. In 2000, NTP co-sponsored a workshop with EPA, "Low Dose Endocrine Disruptors Peer Review." As part of the peer review, a group of statisticians 
reanalyzed several "low" dose studies (Haseman et al. 2001). Based on studies that used littermates, they determined that litter or dam effects were generally present such that pups within a litter were found to respond more similarly than pups from different litters. The overall conclusion was "[f]ailure to adjust for litter effects (e.g., to regard littermates as independent observations and thus the individual pup as the experimental unit) can greatly exaggerate the statistical significance of experimental findings."

\section{Evidence Synthesis}

\section{Endpoint Grouping}

Neurobehavioral endpoints were categorized by nature of behavioral domains using the framework below (Table 4). Findings were synthesized at the test/endpoint level to reach conclusions about potential impacts of fluoride at the domain level (e.g., learning and memory, motor, anxiety). The priority domain in this report is learning and memory.

Table 4. Framework for Neurobehavioral Groupings

\begin{tabular}{ll}
\hline \multicolumn{1}{c}{ General Domain } & \multicolumn{1}{c}{ Example Test and Endpoints } \\
\hline Learning and Memory & $\begin{array}{l}\text { Maze tests (Morris water maze, T-maze, Y-maze); exploration (novel } \\
\text { object recognition, mini-holeboard, activity cage); active and passive } \\
\text { avoidance (step-down test, shuttle box); operant behavior }\end{array}$ \\
Motor and Sensory Function & $\begin{array}{l}\text { Locomotor activity (open field, activity cage); movement coordination } \\
\text { (akinesia/catalepsy, plank walking, rotarod, slanted surface, swim } \\
\text { test); reflex (auditory startle, negative geotaxis, pain response: tail }\end{array}$ \\
& $\begin{array}{l}\text { immersion and Von Frey hair test); developmental motor sensory } \\
\text { landmarks (cliff avoidance, surface righting, pivoting/orienting reflex) }\end{array}$ \\
Fepression & Forced swim; tail suspension test \\
Anxiety & Elevated plus maze \\
Other & Grooming; urination/defecation; sexual behavior; territorial behavior \\
\hline
\end{tabular}

\section{Considerations for Pursuing a Narrative or Quantitative Evidence Synthesis}

Heterogeneity within the available evidence was considered when determining whether to calculate an overall estimate of effect (meta-analysis) of fluoride effects on learning and memory. The principal characteristics evaluated for heterogeneity across eligible studies include the following:

- Animal model used (species, strain, sex, genetic background)

- Age of animals (at start of treatment, mating, pregnancy status)

- Dose levels, frequency of treatment, timing, duration, and exposure route

- Behavioral measurements and methodology

- Type of data (e.g., continuous, dichotomous), statistics presented in paper, ability to access raw data

- Concern for risk of bias 


\section{Standardizing Results from Behavioral Tests and Dose Levels}

Results from behavioral tests were transformed, when possible, to a common metric of percent change from control response to help assess dissimilar but related outcomes measured with different scales. In this project, percent control response was used as the common metric because it is recommended for assessing dissimilar but related outcomes measured with different scales (Vesterinen et al. 2014). Percent control group calculations were based on sample size, means, and standard deviation or standard error values presented in the studies.

For studies in which experimental animals were dosed with sodium fluoride (NaF) or other forms of dissociable fluoride, dose levels were converted to fluoride equivalents $(\mathrm{F})$, for example, $100 \mathrm{ppm} \mathrm{NaF}=45.3 \mathrm{ppm}(\mathrm{mg} / \mathrm{L})$ fluoride. In studies where F was administered directly (often reported simply as "fluoride"), no such conversions were conducted. Fluoride dose levels were standardized to $\mathrm{mg} / \mathrm{kg}-\mathrm{d}$ and $\mathrm{ppm}(\mathrm{mg} / \mathrm{L})$. Conversions were made using water consumption rates and body weights for rats and mice reported in the EPA dosimetry (US EPA 1988; 1994). In each case, the "subchronic" values were chosen because this period fit the maternal or singlegeneration dosing periods in most studies. The strain-specific and sex-specific values were used when available; for strains that were not available, the "other" values were used. For the few studies in which dosing was through the feed, conversion was first made from food ppm to $\mathrm{mg} / \mathrm{kg}$-d. Then, the "effective water concentration" was estimated by multiplying the converted dietary dose by body weight and dividing by water consumption rate. The uncertainty in these estimates should be considered higher than in water consumption studies.

Unless otherwise reported by study authors, the fluoride background level was assumed $0 \mathrm{ppm}$ $(0 \mathrm{mg} / \mathrm{kg}-\mathrm{d})$. Dose levels provided in studies are presented here as $\mathrm{mg} / \mathrm{kg}$-d and ppm as available. Dose conversions were made using US EPA (1988; 1994) default food or water consumption rates and body weights (using subchronic age and experiment duration) for the species/strain and sex of the animal of interest. Dose levels in $\mathrm{mg} / \mathrm{kg}$ - $\mathrm{d}$ can vary for a given $\mathrm{ppm}$ across different studies if the studies use different species/strain or sex of animals, or both, that are assumed to have different food or water consumption rates.

\section{Assessment of Confidence in the Body of Evidence}

The quality of evidence for learning and memory ${ }^{6}$ was evaluated using the GRADE system for rating the confidence in the body of evidence (Guyatt et al. 2011a; NTP 2015a). Under the GRADE system, overall confidence in the body of evidence for an outcome is categorized as high, moderate, low, or very low. Like experimental human studies (randomized clinical trials), evidence from animal studies is initially graded as high quality by OHAT. Next, a series of adjustments ("downgrades" or "upgrades") (Table 5) were made to the initial ranking based on the characteristics of the studies constituting the body of evidence after considering factors. The factors included risk of bias across studies, unexplained inconsistency, indirectness, imprecision, publication bias, effect magnitude, dose response, and consistency across different model systems and study designs (Figure 1, see also NTP (2015a) for additional detail). Studies conducted in mammalian model systems are assumed relevant for humans (i.e., not downgraded for indirectness) unless compelling evidence to the contrary exists.

\footnotetext{
${ }^{6}$ Although level-of-evidence conclusions focused on learning and memory, studies evaluating other aspects of behavior were identified, and data were extracted and assessed for risk of bias (Appendix D through Appendix R).
} 


\begin{tabular}{|c|c|c|c|c|}
\hline \multicolumn{2}{|c|}{$\begin{array}{l}\text { Initial Confidence } \\
\text { by Key Features } \\
\text { of Study Design }\end{array}$} & $\begin{array}{l}\text { Factors } \\
\text { Decreasing } \\
\text { Confidence }\end{array}$ & $\begin{array}{l}\text { Factors } \\
\text { Increasing } \\
\text { Confidence }\end{array}$ & $\begin{array}{l}\text { Confidence } \\
\text { in the Body } \\
\text { of Evidence }\end{array}$ \\
\hline $\begin{array}{l}\text { High }(++++) \\
4 \text { Features }\end{array}$ & Features & $\begin{array}{l}\text { High Risk of } \\
\text { Bias }\end{array}$ & $\begin{array}{l}\text { - Large Magnitude of Effect } \\
\text { - Dose Response }\end{array}$ & High (++++) \\
\hline $\begin{array}{l}\text { Moderate }(+++) \\
3 \text { Features }\end{array}$ & $\begin{array}{l}\text { - Controlled } \\
\text { exposure } \\
\text { - Exposure } \\
\text { prior to } \\
\text { outcome }\end{array}$ & $\begin{array}{l}\text { Unexplained } \\
\text { Inconsistency }\end{array}$ & $\begin{array}{l}\text { Residual Confounding } \\
\text { - Studies report an effect and residual } \\
\text { confounding is toward null }\end{array}$ & Moderate (+++) \\
\hline $\begin{array}{l}\text { Low }(++) \\
2 \text { Features }\end{array}$ & $\begin{array}{l}\text { - Individual } \\
\text { outcome } \\
\text { data } \\
\text { - Comparison } \\
\text { group used }\end{array}$ & $\begin{array}{l}\text { - Indirectness } \\
\text { - Imprecision }\end{array}$ & $\begin{array}{l}\text { Consistency } \\
\text { - Across dissimilar populations }\end{array}$ & Low (++) \\
\hline $\begin{array}{l}\text { Very Low (+) } \\
\leq 1 \text { Features }\end{array}$ & & $\begin{array}{l}\text { Publication } \\
\text { Bias }\end{array}$ & $\begin{array}{l}\text { - Across study design types } \\
\text { Other } \\
\text { - e.g., particularly rare outcomes }\end{array}$ & Very Low (+) \\
\hline
\end{tabular}

\section{NTP descriptions of confidence in the body of evidence categories:}

High: The true effect is highly likely to be reflected in the apparent relationship; further research is very unlikely to change confidence in the apparent relationship. That is, the available evidence usually includes consistent results from well-conducted studies.

Moderate: The true effect may be reflected in the apparent relationship. That is, the available evidence is sufficient to determine an effect is apparent, but our confidence in the estimate is constrained by such factors as:

- The number, size, or quality of individual studies.

- Inconsistency of findings across individual studies.

- $\quad$ Limited generalizability of findings.

As more information becomes available, the magnitude or direction of the observed effect could change, and this change may be large enough to alter the conclusion.

Low: The true effect may be different from the apparent relationship. That is, our confidence is limited and the true effect may be substantially different.

Very low: The true effect is highly likely to be different from the apparent relationship; further research is very likely to have an impact on confidence in the apparent relationship. That is, our confidence is very limited and the available evidence is insufficient to assess effects.

Figure 1. Assessing Confidence in the Body of Evidence ("Quality of Evidence") 
Systematic Literature Review on the Effects of Fluoride on Learning and Memory in Animal Studies

Table 5. Key Factors When Considering Whether to Downgrade or Upgrade

\begin{tabular}{|c|c|}
\hline \multicolumn{2}{|r|}{ Downgrade Factors } \\
\hline Risk of Bias & $\begin{array}{l}\text { Risk of bias across all domains but with priority consideration for these critical } \\
\text { factors: Randomization, blinding at outcome assessment, exposure characterization } \\
\text { (reporting source, purity, internal dose level), control for litter effects in } \\
\text { developmental exposure studies. }\end{array}$ \\
\hline Inconsistency & $\begin{array}{l}\text { Presence of unexplained inconsistency in studies of similar design. Compared to } \\
\text { clinical human data, differences between experimental animal studies could be larger } \\
\text { due to factors such as use of different strains (which are often inbred to minimize } \\
\text { variability), different exposure or treatment paradigms, and differences in handling or } \\
\text { laboratory conditions (Vesterinen et al. 2014). }\end{array}$ \\
\hline \multirow[t]{2}{*}{ Indirectness } & $\begin{array}{l}\text { Within the animal model: Directness of the measure as an indicator of learning and } \\
\text { memory, for example, ability to rule out impaired motor or sensory functions that } \\
\text { might impact ability of animals to perform the learning and memory tests. Also } \\
\text { consider route of administration; oral is considered most relevant for fluoride. }\end{array}$ \\
\hline & $\begin{array}{l}\text { Animal (rodent) to human extrapolation: In vivo mammalian model systems have } \\
\text { demonstrated utility for examining autonomic, sensory, and motor system } \\
\text { functioning as they relate to human health. Although human cognitive function is not } \\
\text { easily assessed in such systems, aspects of learning and memory can be evaluated as } \\
\text { based on learning theory that translates across species (Crawley 2007). Some } \\
\text { neurobehavioral measures, however, have not been demonstrated to translate readily } \\
\text { across species (social behaviors, aggression, risky behaviors) and others (e.g., verbal } \\
\text { learning/performance, gender preferences) cannot be evaluated adequately in a } \\
\text { nonhuman mammalian model system. }\end{array}$ \\
\hline Imprecision & $\begin{array}{l}\text { Confidence in quantitative measures such as effect sizes, identification of no } \\
\text { observed effect level (NOEL) or lowest observed effect level (LOEL) doses, or } \\
\text { parameters for benchmark dose analysis. Typically, } 95 \% \text { confidence intervals are } \\
\text { used as the primary method to assess imprecision (Guyatt et al. 2011b). OHAT also } \\
\text { considers whether studies are adequately powered when considering whether to } \\
\text { downgrade. }\end{array}$ \\
\hline Publication Bias & $\begin{array}{l}\text { Downgrade if "strongly detected." Publication bias is difficult to assess (Guyatt et al. } \\
\text { 2011c), especially when multiple endpoints related to the primary outcome are } \\
\text { reported in the same study, few studies are available, and papers lack reporting on } \\
\text { funding and conflict of interest. Analytical tools, such as funnel plots or trim-and-fill } \\
\text { approaches, can be useful to assess publication bias but also have substantial } \\
\text { limitations and should be interpreted with caution (Guyatt et al. 2011c). }\end{array}$ \\
\hline
\end{tabular}

\section{Upgrade Factors}

Large Magnitude of Effect Determining whether the magnitude of the effect is large includes consideration of factors such as the effect being measured and the dose range used. No upgrade considered if serious concern for risk of bias is present.

Dose Response

Patterns of dose response are evaluated within and across studies when considering whether to upgrade for evidence of dose response. No upgrade considered if serious concern for risk of bias.

Consistency across Animal An upgrade may be considered for consistent results reported in multiple Models or Species experimental animal models or species. 


\section{Preparation of Level-of-evidence Conclusions}

Confidence levels in the body of evidence conclusions from Figure 1 were translated into statements of health effects from animal studies according to one of four statements: (1) High, (2) Moderate, (3) Low, or (4) Inadequate (Figure 2). The descriptor "evidence of no health effect" is used to indicate confidence that the substance is not associated with a health effect as detected by neurobehavioral outcomes. Because of the inherent difficulty in proving a negative, the conclusion "evidence of no health effect" is reached only when confidence in the body of evidence is high.

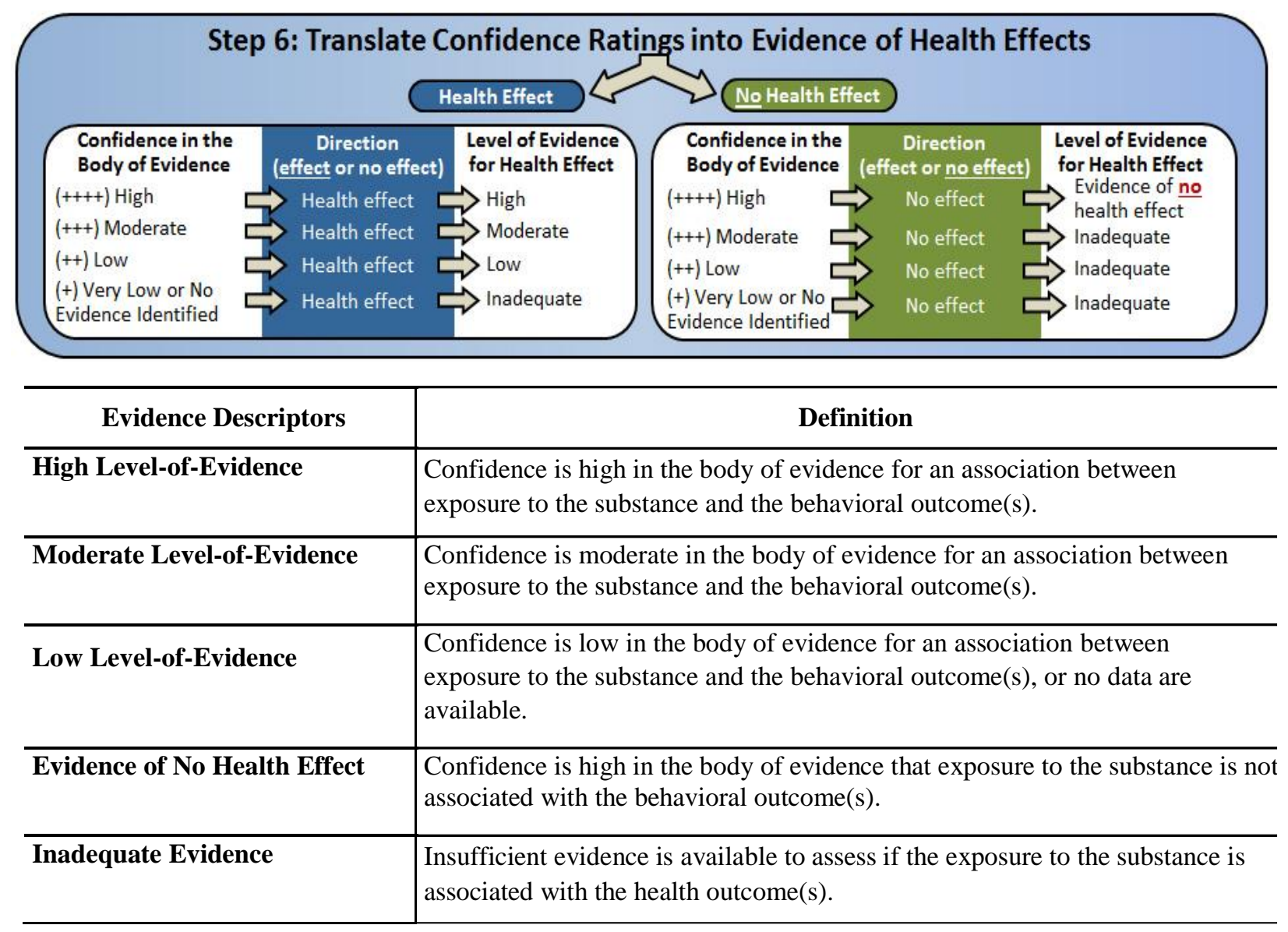

Figure 2. Translate Confidence Ratings into Evidence of Health Effect Conclusions from Animal Studies 


\section{Results}

\section{Literature Search Results}

The database searches yielded 4,643 unique records, and 13 records were identified from reviewing the reference list of included studies or the Fluoride Action Network. ${ }^{7,8}$ Of the 4,656 studies identified, 4,552 were excluded during title and abstract screening, 104 were reviewed at the full-text level, and 68 studies were considered relevant to the PECO statement and were included (Figure 3). Most included studies were published after 2000 (Figure 4). A list of excluded studies is available on the HAWC fluoride project site.

Nineteen of the 68 studies (see Included Studies) were considered to pose a very serious overall risk of bias (Appendix C), primarily based on concern for at least 3 of the following factors: lack of randomization, lack of blinding at outcome assessment in conjunction with not using automated tools to collect information, lack of reporting on what was administered to animals (source, purity, chemical form of fluoride), lack of control for litter effects, lack of expected response in control animals, and lack of reporting of key study information such as the number of animals treated or sex. Across all the studies, information often was not reported and assumed not conducted, resulting in an assessment of "probably high" risk of bias. Authors were queried for missing information, and responses received were used to update risk of bias assessments. Sixteen of the 48 studies that reported findings related to learning and memory were excluded from the evidence synthesis due to concerns for serious risk of bias, which left 32 studies for use in the primary analysis.

\footnotetext{
${ }^{7}$ Thirteen studies were identified as potentially relevant during the reference search of included studies and the Fluoride Action Network website. Two of these were not considered relevant based on full-text review (Bai et al. 2010; Vishnevskii and EL Nichnykh 1969), and we were unable to obtain a full-text record for one (Zhang et al. 2015). Thus, 10 of the 13 records identified from other sources were included.

${ }^{8}$ No relevant records were identified from the chemical toxicity databases after review of dossiers identified using the search term "fluoride." USEPA HPV Challenge Program Robust Summaries and Test Plans: The submission package for "Fatty Nitrogen Derived Amines Category" was reviewed for relevance. European Chemicals Agency (ECHA) Registration dossiers ("REACH"): Study summaries for repeated dose toxicity, reproduction, and developmental toxicity/teratogenicity were reviewed for sodium fluoride, potassium fluoride, calcium fluoride, ammonium fluoride, and magnesium sodium fluoride silicate.
} 
Systematic Literature Review on the Effects of Fluoride on Learning and Memory in Animal Studies

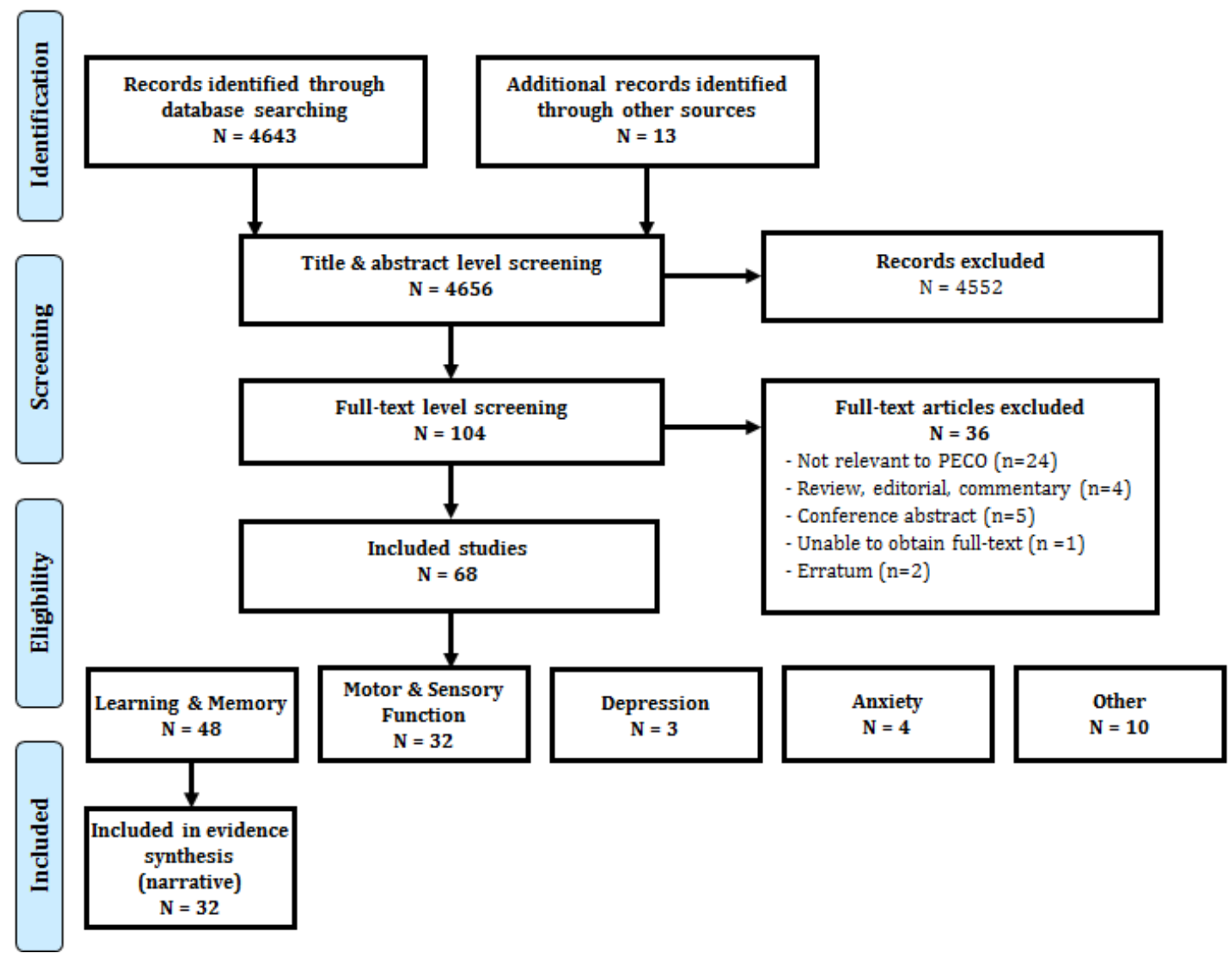

Figure 3. Study Flow Selection Diagram

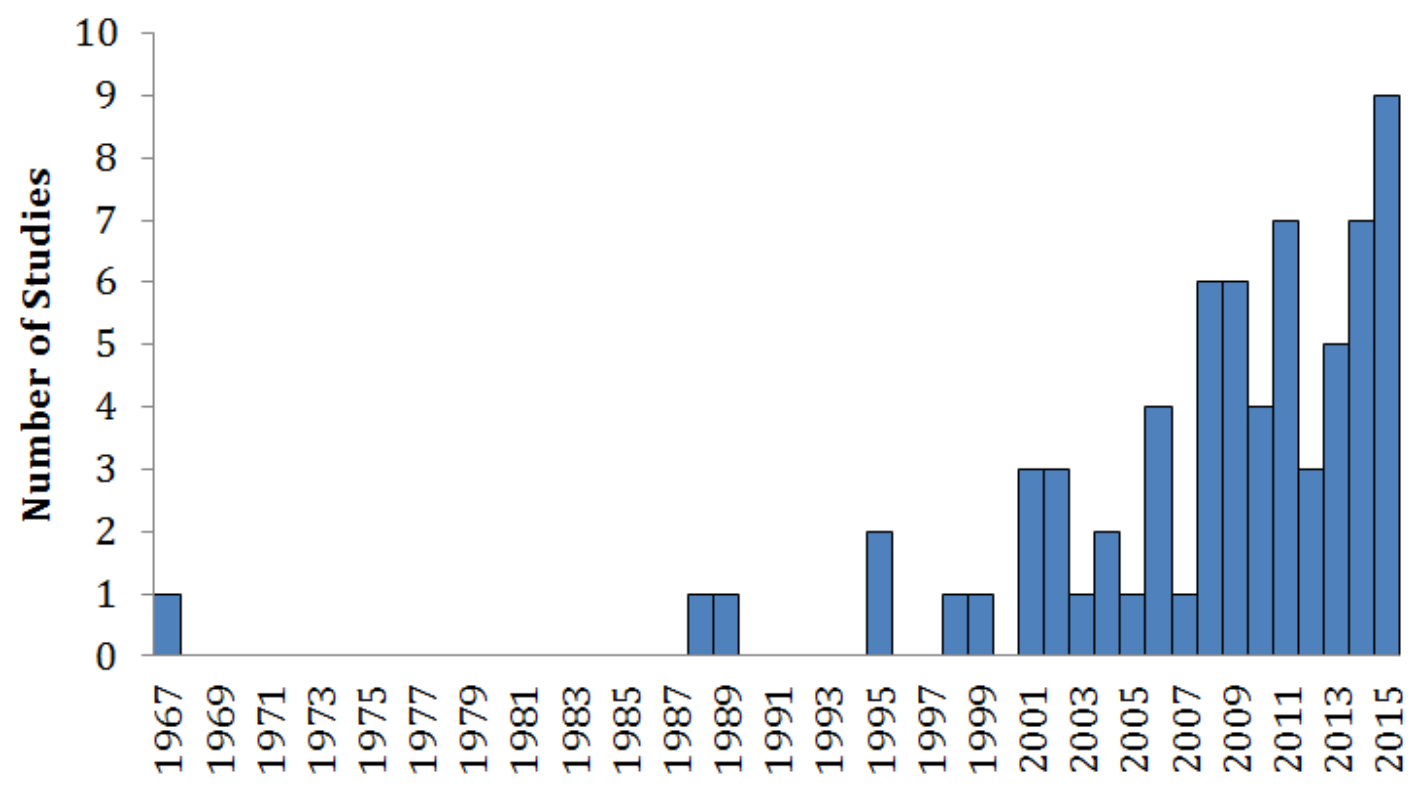

Publication Year

Figure 4. Publication Time Trend 
Systematic Literature Review on the Effects of Fluoride on Learning and Memory in Animal Studies

\section{Included Studies}

1. Balaji B, Kumar EP, Kumar A. 2015. Evaluation of standardized Bacopa monniera extract in sodium fluoride-induced behavioural, biochemical, and histopathological alterations in mice. Toxicol Ind Health. 31(1):18-30.

http://dx.doi.org/10.1177/0748233712468018

2. Balayssac D, Richard D, Authier N, Nicolay A, Jourdan D, Eschalier A, Coudoré F. 2002. Absence of painful neuropathy after chronic oral fluoride intake in SpragueDawley and Lou/C rats. Neurosci Lett. 327(3):169-172. http://dx.doi.org/10.1016/S03043940(02)00421-4

3. Banji D, Banji OJ, Pratusha NG, Annamalai A. 2013. Investigation on the role of Spirulina platensis in ameliorating behavioural changes, thyroid dysfunction and oxidative stress in offspring of pregnant rats exposed to fluoride. Food Chem. 140(12):321-331. http://dx.doi.org/10.1016/j.foodchem.2013.02.076

4. Baran-Poesina V, Negres S, Dobrescu D, Dimcevici-Poesina N, Dimcevici-Poesina A, Feghiu A, Soare T, Militaru M. 2013. Experimental pharmacological researches regarding the influence of sodium fluoride in allopathic and homeopathic doses on central nervous system's performances. A correlation between behavioral response in classic maze test and morphological aspects of cerebral cortex. Farmacia. 61(4):781-799.

5. Bartos M, Gumilar F, Bras C, Gallegos CE, Giannuzzi L, Cancela LM, Minetti A. 2015. Neurobehavioural effects of exposure to fluoride in the earliest stages of rat development. Physiol Behav. 147:205-212. http://dx.doi.org/10.1016/j.physbeh.2015.04.044

6. Basha PM, Rai P, Begum S. 2011. Fluoride toxicity and status of serum thyroid hormones, brain histopathology, and learning memory in rats: a multigenerational assessment. Biol Trace Elem Res. 144(1-3):1083-1094. http://dx.doi.org/10.1007/s12011011-9137-3

7. Basha PM, Sujitha N. 2012. Combined impact of exercise and temperature in learning and memory performance of fluoride toxicated rats. Biol Trace Elem Res. 150(1-3):306313. http://dx.doi.org/10.1007/s12011-012-9489-3

8. Bataineh HN, Nusier MK. 2006. Impact of 12-week ingestion of sodium fluoride on aggression, sexual behavior, and fertility in adult male rats. Fluoride. 39(4):293-301.

9. Bera I, Sabatini R, Auteri P, Flace P, Sisto G, Montagnani M, Potenza MA, Marasciulo FL, Carratu MR, Coluccia A et al. 2007. Neurofunctional effects of developmental sodium fluoride exposure in rats. Eur Rev Med Pharmacol Sci. 11(4):211-224.

10. Bhatnagar M, Rao P, Jain S, Bhatnagar R. 2002. Neurotoxicity of fluoride: neurodegeneration in hippocampus of female mice. Indian J Exp Biol. 40(5):546-554.

11. [Chen H, Geng D. 2011. The change of cognition induced by chronic fluoride in rats. Acta Academiae Medicinae Xuzhou 31(5):319-322.]*

12. Chioca LR, Raupp IM, Da Cunha C, Losso EM, Andreatini R. 2008. Subchronic fluoride intake induces impairment in habituation and active avoidance tasks in rats. Eur $\mathbf{J}$ Pharmacol. 579(1-3):196-201. http://dx.doi.org/10.1016/j.ejphar.2007.10.019 
Systematic Literature Review on the Effects of Fluoride on Learning and Memory in Animal Studies

13. [Dong Y-T, Wang Y, Wei N, Guan Z-Z. 2015. Expression levels of brain muscarinic acetylcholine receptor in offspring rats of drinking-water borne fluorosis. Chinese Journal of Endemiology. 34(5):326-330.]

14. [Dong Y-T, Wang Y, Wei N, Guan Z-Z. 2015. Expression of muscarinic acetylcholine receptors in the brain of rats with chronic fluorosis. Chinese Journal of Endemiology. 34(2):84-88.]

15. Dong Y-T, Wang Y, Wei N, Zhang Q-F, Guan Z-Z. 2015. Deficit in learning and memory of rats with chronic fluorosis correlates with the decreased expressions of M1 and M3 muscarinic acetylcholine receptors. Arch Toxicol. 89(11):1981-1991. http://dx.doi.org/10.1007/s00204-014-1408-2

16. Ekambaram P, Paul V. 2001. Calcium preventing locomotor behavioral and dental toxicities of fluoride by decreasing serum fluoride level in rats. Environ Toxicol Pharmacol. 9(4):141-146. http://dx.doi.org/10.1016/S1382-6689(00)00063-6

17. Ekambaram P, Paul V. 2002. Modulation of fluoride toxicity in rats by calcium carbonate and by withdrawal of fluoride exposure. Pharmacol Toxicol. 90(2):53-58. http://dx.doi.org/10.1034/j.1600-0773.2002.900201.x

18. Ekambaram P, Paul V. 2003. Effect of vitamin D on chronic behavioral and dental toxicities of sodium fluoride in rats. Fluoride. 36(3):189-197.

19. El-lethey H, Kamel M, Shaheed I. 2011. Perinatal exposure to sodium fluoride with emphasis on territorial aggression, sexual behaviour and fertility in male rats. Life Science Journal-Acta Zhengzhou University Overseas Edition 8(2):686-694.

20. El-lethey HS, Kamel MM. 2011. Effects of black tea in mitigation of sodium fluoride potency to suppress motor activity and coordination in laboratory rats. J Am Sci. 7:243$254{ }^{*}$

21. El-lethey HS, Kamel MM, Shaheed IB. 2010. Neurobehavioral toxicity produced by sodium fluoride in drinking water of laboratory rats. J Am Sci. 6(5):54-63.*

22. El-lethey HS, Shaheed IB. 2011. Potential health impact of black tea against Na-FInduced alterations in territorial aggression, sexual behaviour and fertility of male rats. Life Sci J. 8:828-839.

23. Elliott L. 1967. Lack of effect of administration of fluoride on the central nervous system of rats. Acta Pharmacol Toxicol (Copenh) 25(3):323-328. http://dx.doi.org/10.1111/j.1600-0773.1967.tb01439.x

24. Flace P, Benagiano V, Vermesan D, Sabatini R, Inchingolo A, Auteri P, Ambrosi G, Tarullo A, Cagiano R. 2010. Effects of developmental fluoride exposure on rat ultrasonic vocalization, acoustic startle reflex and pre-pulse inhibition. Eur Rev Med Pharmacol Sci. 14(6):507-512.

25. [Gao Q, Liu YJ, Guan ZZ, Wu CX, Long YG. 2008. Level of oxidative stress in rat brains and learning and memory function of rats with chronic fluorosis. Chinese Journal of Endemiology. 27(4):371-373.]

26. [Gao Q, Liu YJ, Wu CX, Long YG, Guan ZZ. 2008. Effects of fluoride on learning and memory and cholinesterase activity in rat brains. Chinese J Endemiology. 27(2):128130.] 
Systematic Literature Review on the Effects of Fluoride on Learning and Memory in Animal Studies

27. Gao Q, Liu Y-J, Guan Z-Z. 2009. Decreased learning and memory ability in rats with fluorosis: increased oxidative stress and reduced cholinesterase activity in the brain. Fluoride. 42(4):277-285.

28. [Gao YL, Liu L, Young L, Huan LZ, Jin HH. 2009. Effects of learning and memory of fluoride and the antagonism of selenium in rats. Studies of Trace Elements and Health. 26:1-3.]*

29. Gopal K, Saxena R, Gupta G, Rana M, Agrawal D. 2006. Fluoride induced alterations in neurobehavioural and cardiovascular responses in rats. J Advanced Zool 27(1):1-7.

30. Gui C-Z, Ran L-Y, Li J-P, Guan Z-Z. 2010. Changes of learning and memory ability and brain nicotinic receptors of rat offspring with coal burning fluorosis. Neurotoxicol Teratol. 32(5):536-541. http://dx.doi.org/10.1016/j.ntt.2010.03.010

31. Han H, Du W, Zhou B, Zhang W, Xu G, Niu R, Sun Z. 2014. Effects of chronic fluoride exposure on object recognition memory and mRNA expression of SNARE complex in hippocampus of male mice. Biol Trace Elem Res. 158(1):58-64. http://dx.doi.org/10.1007/s12011-014-9889-7

32. [Hong JH, Ge YM, H.M. N. 2005. Effects of High Fluoride and Low Iodine on LearningMemory and TchE of Brain in Offspring Rats. China Preventive Medicine. 6:489-491.]*

33. Jain A, Mehta VK, Chittora R, Mahdi AA, Bhatnagar M. 2015. Melatonin ameliorates fluoride induced neurotoxicity in young rats: An In Vivo evidence. Asian Journal of Pharmaceutical and Clinical Research. 8(4):164-167.

34. Jetti R, Raghuveer C, Rao C. 2016. Protective effect of ascorbic acid and Ginkgo biloba against learning and memory deficits caused by fluoride. Toxicol Ind Health. 32(1):183187. http://dx.doi.org/10.1177/0748233713498460

35. Jiang C, Zhang S, Liu H, Guan Z, Zeng Q, Zhang C, Lei R, Xia T, Wang Z, Yang L. 2014. Low glucose utilization and neurodegenerative changes caused by sodium fluoride exposure in rat's developmental brain. Neuromolecular Med. 16(1):94-105. http://dx.doi.org/10.1007/s12017-013-8260-Z

36. Jiang S, Su J, Yao S, Zhang Y, Cao F, Wang F, Wang H, Li J, Xi S. 2014. Fluoride and arsenic exposure impairs learning and memory and decreases mGluR5 expression in the hippocampus and cortex in rats. PloS one. 9(4):e96041. http://dx.doi.org/10.1371/journal.pone.0096041

37. Kivrak Y. 2012. Effects of fluoride on anxiety and depression in mice. Fluoride. 45(3):302-306.

38. Li M, Cui J, Gao Y, Zhang W, Sun L, Liu X, Liu Y, Sun D. 2015. Pathological changes and effect on the learning and memory ability in rats exposed to fluoride and aluminum. Toxicol Res. 4(5):1366-1373. http://dx.doi.org/10.1039/C5TX00050E

39. Liu F, Ma J, Zhang H, Liu P, Liu Y-P, Xing B, Dang Y-H. 2014. Fluoride exposure during development affects both cognition and emotion in mice. Physiol Behav. 124:1-7.

40. [Liu WX. 1989. Experimental study of behavior and cerebral morphology of rat pups generated by fluorotic female rat. Zhonghua Bing Li Xue Za Zhi 18(4):290-292.] 
Systematic Literature Review on the Effects of Fluoride on Learning and Memory in Animal Studies

41. [Liu YJ, Gao Q, Long YG, Yu YN, Guan ZZ. 2011. Influence of chronic fluorosis on expression of phospho-Elk-1 in rat brains. Chinese Journal of Endemiology. 30(3):251255.]

42. [Liu YJ, Gao Q, Wu CX, Long YG, Guan ZZ. 2009. Modified expression of extracellular signal-regulated protein kinase signal transduction in rat brains and changed capacity of learning and memory of rats with chronic fluorosis. Chinese J Endemiology. 28(1):3235.]

43. Liu Y-J, Gao Q, Wu C-X, Guan Z-Z. 2010. Alterations of nAChRs and ERK1/2 in the brains of rats with chronic fluorosis and their connections with the decreased capacity of learning and memory. Toxicol Lett. 192(3):324-329. http://dx.doi.org/10.1016/j.toxlet.2009.11.002

44. Ma J, Liu F, Liu P, Dong Y-Y, Chu Z, Hou T-Z, Dang Y-H. 2015. Impact of early developmental fluoride exposure on the peripheral pain sensitivity in mice. International Journal of Developmental Neuroscience: the official journal of the International Society for Developmental Neuroscience. 47(Part B):165-171. http://dx.doi.org/10.1016/j.ijdevneu.2015.09.005

45. Mullenix PJ, Denbesten PK, Schunior A, Kernan WJ. 1995. Neurotoxicity of sodium fluoride in rats. Neurotoxicol Teratol. 17(2):169-177. http://dx.doi.org/10.1016/08920362(94)00070-T

46. Niu R, Liu S, Wang J, Zhang J, Sun Z, Wang J. 2014. Proteomic analysis of hippocampus in offspring male mice exposed to fluoride and lead. Biol Trace Elem Res. 162(1-3):227-233. http://dx.doi.org/10.1007/s12011-014-0117-2

47. Niu R, Sun Z, Cheng Z, Li Z, Wang J. 2009. Decreased learning ability and low hippocampus glutamate in offspring rats exposed to fluoride and lead. Environ Toxicol Pharmacol. 28(2):254-258. http://dx.doi.org/10.1016/j.etap.2009.04.012

48. Niu R, Sun Z, Wang J, Cheng Z, Wang J. 2008. Effects of fluoride and lead on locomotor behavior and expression of Nissl body in brain of adult rats. Fluoride. 41(4):276-282.

49. Paul V, Ekambaram P, Jayakumar A. 1998. Effects of sodium fluoride on locomotor behavior and a few biochemical parameters in rats. Environ Toxicol Pharmacol. 6(3):187-191. http://dx.doi.org/10.1016/S1382-6689(98)00033-7

50. Pereira M, Dombrowski PA, Losso EM, Chioca LR, Da Cunha C, Andreatini R. 2011. Memory impairment induced by sodium fluoride is associated with changes in brain monoamine levels. Neurotox Res. 19(1):55-62. http://dx.doi.org/10.1007/s12640-0099139-5

51. Raghu J, Raghuveer VC, Rao MC, Somayaji NS, Babu PB. 2013. The ameliorative effect of ascorbic acid and Ginkgo biloba on learning and memory deficits associated with fluoride exposure. Interdiscip Toxicol. 6(4):217-221. http://dx.doi.org/10.2478/intox2013-0032

52. Reddy MM, Karnati PR. 2015. Protective effects of aqueous extract of fruit pulp of Tamarindus indica on motor activity and metabolism of the gastrocnemius muscle of rats treated with fluoride. International Journal of Toxicological and Pharmacological Research. 7(5):241-246. 
Systematic Literature Review on the Effects of Fluoride on Learning and Memory in Animal Studies

53. [Rumiantsev G, Novikov S, Mel'nikova N, Levchenko N, Kozeeva E. 1988. Experimental study of the biological effect of salts of hydrofluosilicic acid. Gigiena $\mathrm{i}$ sanitariia. (11):80-82.]

54. Sarkozi K, Horvath E, Vezer T, Papp A, Paulik E. 2015. Behavioral and general effects of subacute oral arsenic exposure in rats with and without fluoride. Int J Environ Health Res. 25(4):418-431. http://dx.doi.org/10.1080/09603123.2014.958138

55. [Shen X, Zhang Z, Xu X. 2004. Effect of iodine and selenium on learning memory impairment induced by fluorosis and blood biochemical criterion of rats. Occupation and Health. 20:6-8.]*

56. Sun ZR, Liu FZ, Wu LN, Lu Y, Yu DK. 2008. Effects of high fluoride drinking water on the cerebral function of mice. Fluoride. 41(2):148-151.

57. [Wang G, Li J, Zhu H, Zhu. 2006. Effect of different doses of chronic exposure of fluoride on rat learning and memory behavior Studies of Trace Elements and Health $23: 1-2.]^{*}$

58. Wang J, Ge Y, Ning H, Wang S. 2004. Effects of high fluoride and low iodine on biochemical indexes of the brain and learning-memory of offspring rats. Fluoride. 37(3):201-208.

59. [Wei N, Dong Y, Wang Y, Guan Z. 2014. Effects of chronic fluorosis on neurobehavioral development in offspring of rats and antagonistic effect of Vitamin E. Chinese Journal of Endemiology. 33(2):125-128.]

60. Whitford GM, Whitford JL, Hobbs SH. 2009. Appetitive-based learning in rats: lack of effect of chronic exposure to fluoride. Neurotoxicol Teratol. 31(4):210-215. http://dx.doi.org/10.1016/j.ntt.2009.02.003

61. Wu C, Gu X, Ge Y, Zhang J, Wang J. 2006. Effects of high fluoride and arsenic on brain biochemical indexes and learning-memory in rats. Fluoride. 39(4):274-279.

62. Wu NP, Zhao ZL, Gao W, Li XQ. 2008. Behavioral teratology in rats exposed to fluoride. Fluoride. 41(2):129-133.

63. [Xu X, Shen X. 2001. Effect of fluorosis on mice learning and memory behaviors and brain SOD activity and MDA content. China Public Health 17:8-10.]*

64. Zhang C, Ren C, Chen H, Geng R, Fan H, Zhao H, Guo K, Geng D. 2013. The analog of Ginkgo biloba extract 761 is a protective factor of cognitive impairment induced by chronic fluorosis. Biol Trace Elem Res. 153(1-3):229-236.

65. [Zhang J, Zhang Z. 2009. The effect of fluorine exposure of pregnant rats on the learning and memory capabilities of baby rats Chinese Journal of Public Health 25:1347-1348.]*

66. [Zhang Z, Shen X, Xu X. 2001. Effects of selenium on the damage of learning-memory ability of mice induced by fluoride. Wei Sheng Yan Jiu. 30(3):144-146.]

67. [Zhang Z, Xu X, Shen X. 1999. Effect of fluoride exposure on synaptic structure of brain areas related to learning-memory in mice. Wei Sheng Yan Jiu. 28(4):210-212.]

68. [Zhu YL, Zheng YJ, Ma Y, Zhang J. 2012. Effects of fluoride exposure on performance in water labyrinth and monoamine neurotransmitters of rats. Journal of Xinjiang Medical University. 35.]*

*Identified from other sources, [ ] non-English. 


\section{Overview of Included Studies}

A survey of included studies describing species, lifestage at exposure, and dose levels tested is presented in Table 6. Eighteen of the 68 studies (26\%) were in non-English, defined as those for which a publicly available version in English was not available. ${ }^{9}$ English-translated versions of several studies were available in the publication Fluoride and are not classified as non-English.

Table 6. Description of Relevant Studies

\begin{tabular}{|c|c|c|c|c|c|}
\hline Descriptor & $\begin{array}{c}\text { Learning and } \\
\text { Memory }\end{array}$ & $\begin{array}{c}\text { Motor and Sensory } \\
\text { Function } \\
\end{array}$ & Depression & Anxiety & Other $^{1}$ \\
\hline Number of Studies & 48 & 32 & 3 & 4 & 10 \\
\hline Non-English & $17(35 \%)$ & $6(19 \%)$ & $0(0 \%)$ & $0(0 \%)$ & $0(0 \%)$ \\
\hline \multicolumn{6}{|l|}{ Species } \\
\hline Rats & 39 & 24 & 0 & 2 & 8 \\
\hline Mice & 9 & 8 & 3 & 2 & 2 \\
\hline \multicolumn{6}{|l|}{ Lifestage of Exposure ${ }^{2}$} \\
\hline Adult & 33 & 22 & 3 & 2 & 5 \\
\hline Developmental & 16 & 11 & 0 & 2 & 5 \\
\hline Doses Range Tested ${ }^{2}$ & 0.45-271 ppm & $0.9-226 \mathrm{ppm}$ & 0.9-90 ppm & $0.9-90 \mathrm{ppm}$ & $1-136 \mathrm{ppm}$ \\
\hline Developmental & $1-200 \mathrm{ppm}(\mathrm{n}=16)^{3}$ & $1-68 \mathrm{ppm}(\mathrm{n}=11)$ & - & $\begin{array}{l}2.26-61 \mathrm{ppm} \\
\quad(\mathrm{n}=2)\end{array}$ & $1-45 \mathrm{ppm}(\mathrm{n}=5)$ \\
\hline$\leq 5 \mathrm{ppm}$ & 2 studies & 3 studies & - & 1 study & 2 studies \\
\hline$\leq 4 \mathrm{ppm}$ & 2 studies & 3 studies & - & 1 study & 2 studies \\
\hline Adult & $\begin{array}{l}0.45-271 \mathrm{ppm} \\
\quad(\mathrm{n}=33)\end{array}$ & $0.9-226 \mathrm{ppm}(\mathrm{n}=22)$ & $\begin{array}{c}0.9-90 \mathrm{ppm} \\
(\mathrm{n}=3)\end{array}$ & $\begin{array}{c}0.9-90 \mathrm{ppm} \\
(\mathrm{n}=2)\end{array}$ & $\begin{array}{c}40-136 \mathrm{ppm} \\
(\mathrm{n}=5)\end{array}$ \\
\hline$\leq 5 \mathrm{ppm}$ & 12 studies & 2 studies & 1 study & 1 study & 0 studies \\
\hline$\leq 4 \mathrm{ppm}$ & 12 studies & 2 studies & 1 study & 1 study & 0 studies \\
\hline \multicolumn{6}{|c|}{ Studies with Very Serious Risk of Bias ${ }^{4}$} \\
\hline Total & $16 / 48$ & $9 / 32$ & 0 & 0 & $3 / 10$ \\
\hline Developmental & $9 / 16$ & $5 / 11$ & - & 0 & $2 / 5$ \\
\hline$\leq 5 \mathrm{ppm}$ & $2 / 2(100 \%)$ & $2 / 3(66 \%)$ & - & - & - \\
\hline$\leq 4 \mathrm{ppm}$ & $2 / 2(100 \%)$ & $2 / 3(66 \%)$ & - & - & - \\
\hline Adult & $7 / 33$ & $4 / 22$ & 0 & 0 & $1 / 5$ \\
\hline$\leq 5 \mathrm{ppm}$ & $2 / 12(17 \%)$ & 0 & 0 & 0 & 0 \\
\hline$\leq 4 \mathrm{ppm}$ & $2 / 12(17 \%)$ & 0 & 0 & 0 & 0 \\
\hline \multicolumn{6}{|c|}{ Studies Used for Evidence Synthesis } \\
\hline Total & 32 & - & - & - & - \\
\hline Developmental & $7(11-50 \mathrm{ppm})$ & - & - & - & - \\
\hline$\leq 5 \mathrm{ppm}$ & 0 & - & - & - & - \\
\hline$\leq 4 \mathrm{ppm}$ & 0 & - & - & - & - \\
\hline Adult & $26(0.45-226 \mathrm{ppm})$ & - & - & - & - \\
\hline$\leq 5 \mathrm{ppm}$ & 10 & - & - & - & - \\
\hline$\leq 4 \mathrm{ppm}$ & 10 & - & - & - & - \\
\hline
\end{tabular}

${ }^{1}$ Other includes studies assessing grooming/urination/defecation; sexual behavior, territorial aggression, vocalization.

${ }^{2}$ Expressed in ppm fluoride equivalents. The number of studies might not total because studies often tested multiple dose levels and some studies evaluated effects in multiple lifestages of exposure.

${ }^{3}$ Duplicate data from Dong et al. (2015c) appear to be reported in a second study from this group Dong et al. (2015a).

${ }^{4}$ See Appendix C.

${ }^{9}$ (Chen and Geng 2011; Dong et al. 2015a; Dong et al. 2015b; Gao et al. 2008a; Gao et al. 2008b; Gao et al. 2009b; Hong et al. 2005; Liu 1989; Liu et al. 2011; Liu et al. 2009; Rumiantsev et al. 1988; Shen et al. 2004; Wang et al. 2006; Wei et al. 2014; Xu et al. 2001; Zhang et al. 2009; Zhang et al. 2001; Zhu et al. 2012). 


\section{Behavioral Tests for Learning and Memory}

Theories of learning stress the significance of drives and incentives or motivating factors in the process of acquisition and in establishing a memory of a task. In animal experimentation, there are a variety of motivating factors, including such incentives as escape from punishment, escape from water, satisfaction of hunger, satisfaction for novel experiences, sexual satisfaction, satisfaction of maternal drive, and satisfaction to return to home cage and cage mates. A relative efficacy exists for different degrees of reward and the degree-of-reward-value inherent in any incentive can be modified by varying the physiological condition of the animal (e.g., periods of deprivation).

All learning paradigms that rely on appetitive reinforcement (food or water) require animals to be placed on deprivation to increase reinforcement value. Once animals have learned to perform the task, with continued training to examine full acquisition or to transition to a more complicated paradigm, body weight is normally maintained at $80-85 \%$ of free feeding body weight. Although initially this condition might be stressful to the animals, the restricted caloric intake can be of health benefit. For toxicological assessments, consideration is required with regard to the initial health of the animals and the motivational incentive value of the appetitive reward. The use of aversive stimuli, which for the animal is reinforced by removing itself from experiencing, is by its nature stressful in the initial acquisition phase. The reinforcement value of the stimuli is a variable that requires consideration. For example, altering the pain threshold (diminished or elevated) will influence reinforcement value of a painful stimulus and, thus, acquisition.

The reliance on a motor response can result in issues that require consideration for interpretation. As an example, if latency is an outcome endpoint, absence of general motor performance equivalency could become an issue for concern. In maze-based tasks, latency often determines performance. In these cases, altered motor function or activity levels can significantly influence the outcome endpoint yet not reflect differences in learning. In avoidance tasks, for animals that display lower activity levels, performance on passive avoidance tasks can appear improved while animals with a higher level of activity can give the impression of poor performance, neither of which would directly reflect learning capability. The reverse is true for active avoidance paradigms.

Animals can display differences in response characteristic or level. For example, an animal might respond to the negative stimuli by freezing rather than escaping or might find an alternative method to endure the stimuli. These differences in response do not necessarily mean the animal has not learned the associations between cues-just that it is not performing as the test paradigm dictates. In various tests for learning and memory, the assessment of memory might inadvertently impose an extinction trial that would weaken the conditioned association between cues and influence any subsequent test session. As in all learning assessments, an understanding is required of the learning process and the manifestation of a learned behavior in an individual animal that differs from the defined response of the paradigm.

The following examples of test paradigms used to assess learning and memory in rodents are not intended to be inclusive. Rather, they reflect the tests represented in the literature for evaluating the effects of fluoride exposure on learning and memory. 


\section{Morris Water Maze}

The Morris water maze is a standardized test used to evaluate aspects of spatial learning and memory in rats and mice (Vorhees and Williams 2006; 2014). The general paradigm is to allow the animal to navigate a circular water-filled tank to find a hidden platform that will allow for escape from the water. The animal accomplishes this task by using various visual cues within the spatial environment. The animal is allowed to search the tank for a defined period (60-120 seconds) to find the platform. Several sequential training trials are conducted (the most common is four trials per day). Several sequential daily training sessions are conducted consisting of multiple trials per day to reach a predetermined criterion of learning. Learning, as defined by acquisition of the task over training sessions, is evaluated by a decrease in the amount of time the animal takes to find the platform (latency), a decrease in the distance traveled to reach the platform, and (with video imaging techniques) a shift in the swimming pattern to achieve a more direct and efficient route to the platform. Additional measures collected include visual capability (seeing a visual platform above the water surface) and swimming speed across multiple sessions to confirm an absence of differences between groups that could affect performance on the Morris water maze and influence data interpretation regarding learning and memory. When the animal reaches a set criterion of performance for the Morris water maze (has learned the task), a spatial memory assessment (probe trial) is conducted. During this probe trial, the platform is removed and the search strategy of the animal is evaluated [time spent in escape platform quadrant (absolute and relative to each other quadrant), latency and distance traveled to the first time crossing the previous platform location, number of times the animal crosses the previous platform location]. These measures are collected across the total time interval for the probe trial (60-90 seconds) to capture the memory of the previous platform location (memory) and within equal time intervals (15-sec epochs) to capture the animal's ability to detect the absence of the platform and to change the search strategy, respectively. To confirm a learning deficit, a "reversal-learning" session can follow the probe trial in which the animal is required to learn a new location of the escape platform. Motor strength, motor coordination, or attention can influence any isolated measures of latency or distance traveled, rather than acquisition (better performance over time), and thus, confound interpretation of change in learning. Many studies examining the effect of fluoride exposure on Morris water maze performance relied on such isolated measures, and the acquisition of the task was not analyzed. In addition, endpoints collected during the memory probe tests often were limited to motor-dependent tasks (e.g., latency to the initial find of the learned location of the previously hidden platform, number of times the animal swam across the platform site during a single trial). Few studies included an assessment of the time an animal spent in the "platform" location.

\section{T-Maze}

The T-maze is an apparatus shaped like the letter $\mathrm{T}$ that can be used to evaluate exploratory activity (in absence of a reinforcement) and, in a variety of ways, to evaluate learning and memory in experimental animals. Typically, a spontaneous alternation paradigm and a reinforced alternation paradigm (working memory) were used in the fluoride studies. Normal control animals (mice and rats) display a tendency to alternate their choice of an arm of the maze to enter (e.g., an entry into the right arm on trial 1 is highly likely to be followed by an entry into the left arm of the subsequent trial). In the absence of reinforcement, this behavior is termed spontaneous alteration. The normal alternating pattern is examined over trials in quick succession as a measure that the animal remembers the arm visited in the previous session. With reinforcement 
(positive or negative), the paradigm is termed a reinforced alternation paradigm. The animal is placed at the start location in the bottom of the $\mathrm{T}$ and allowed to traverse the alley of the maze, making a choice of turning right or left. One arm of the maze, which contains reinforcement, is considered the "correct" arm. The animal is removed from the maze and a subsequent trial conducted. Training consists of a defined number of trials within any one session and several sessions per day for a defined number of days, usually until the animals reach a previously defined criterion of the percentage of correct choices. The number of correct choices and latency to run the maze from the start location to the goal box is recorded. Acquisition is normally determined by a decrease in percentage of incorrect choices and a decrease in latency over time. Deficits in motor function can influence latency, and motivation level of the animal can influence latency and percentage of correct choices.

A multiple T-maze test is a complex maze having multiple T-junctions. Performance is measured as a right or wrong choice at each $\mathrm{T}$ intersection. Multiple $\mathrm{T}$-mazes are used to address questions of place versus response learning and cognitive maps. A mouse or rat is placed in the maze and allowed to explore freely for a defined period across a few trials. Under food-deprivation conditions, the animal is placed in one T-end of the maze and allowed to explore the maze for a defined period to find the food reinforcement. This reinforced trial is repeated. Acquisition of the task is measured as a decrease in latency to obtain the reward and a decrease in percentage of incorrect choices at junctions. Motivation level and anxiety/fear level of the animal can influence latency and percentage of correct choices.

\section{Y-Maze}

The Y-maze is an apparatus with three arms, oriented at 120-degree angles from each other that can be used to assess exploratory behavior (animals tend to enter a less recently visited arm) or can be used, in conjunction with cued reinforcement, to examine learning and memory for selecting arm to enter. A Y-maze paradigm is similar to the T-maze paradigm in that it can record spontaneous alternation of arm entered or arms entered for reinforcement (positive or negative). A sequence of trials consists of placing the animal in the start location at the base of the maze and allowing the animal to traverse the length of the maze. Over trials, the sequence of choices is recorded to identify the pattern of alternation from one arm to the other. In controls, the choice sequence is expected to alternate with each trial under nonreinforced conditions (spontaneous alternation). For reinforced alternation, the animal's entering a specific arm (either the same or different) is reinforced with each trial, depending on the test paradigm. Acquisition of the task is measured as a decrease in the number of choice errors (entering the incorrect arm). Latency from the start box to the goal box or to the choice point also can be recorded.

Differences in motor function or motor performance can influence latency. Motivation level and anxiety/fear level of the animal can influence latency and percentage of correct choices.

\section{Classic Maze}

Classic maze performance is related to the animal's progression over sessions to increase its performance as measured by latency or number of errors, depending on the maze design and paradigm. Although its design can vary, the classic maze generally requires the animal to learn to make a series of correct choices (turns) to navigate the maze successfully and receive reinforcement. Appetitive reinforcement, such as food reward, can be used with animals maintained at a level of restricted feeding to enhance motivation. Negative reinforcement also can be used. A standard paradigm for a classic maze involves the animal's being placed in the 
start location of the maze and allowed to explore the maze freely for a defined period. A series of successive trials follows during which the animal learns to navigate the maze more quickly and correctly. Acquisition of the task is reflected as a decrease over trials in latency (time) to reach reinforcement or the number of times the animal makes an error in selecting the correct direction

to take at a choice point, or both. Differences in motor function can influence latency. Motivation level of the animal and anxiety/fear level can influence latency and percentage of correct choices.

\section{Passive Avoidance}

Passive avoidance is a fear-motivated test used to evaluate associative learning and memory. An animal is required to learn to withhold a normal preferred response such as moving from a brightly illuminated large chamber into a smaller dark chamber or moving from an elevated platform to a lower floor. A standard paradigm involves the animal's being placed in the nonpreferred side of the apparatus. The animal is allowed to explore the apparatus freely and, upon the first entry in the normally preferred side, a negative stimulus is delivered. The animal undergoes two or three training/test sessions. The strength of the learned association is indicated by an increase in latency to leave the "safe" chamber and enter the "non-safe" chamber over sessions. The strength of the association can be examined by eliminating the adverse stimuli and measuring the number of trials required for the animal to return to a pretraining latency to enter the "non-safe" side. The strength of the reinforcement (e.g., perception of pain from the shock) influences the strength of the learning. Motor activity level can influence latency, with a decreased activity level skewing the response to appear as if a greater level of learning occurred, while hyperactivity would cause the animal to move prematurely and thus, imply a deficit in learning.

\section{Active Avoidance}

Active avoidance is a fear-motivated test used to evaluate associative learning and memory. Animals learn to associate a conditioned stimulus (light, tone) with the delivery of an unconditioned negative stimulus (e.g., shock) and to move to a safe location to escape (escape response) or avoid (avoidance response) the negative stimulus. A standard paradigm involves the animal's being placed in the test apparatus and allowed to explore the environment freely. A training trial is initiated by the delivery of a cue (light), followed within a defined time interval (5-10 sec as determined by the test parameters) by the presentation of a second cue (tone) paired with a shock (or other strong negative stimulus). The animal can escape the negative stimulus by moving from the "non-safe" environment upon the delivery of the first cue (avoidance) or with the second cue and shock (escape). With training, the animal learns an association between the cue (tone, conditioned stimulus) and the shock (unconditioned stimulus). Acquisition is demonstrated with an increase in avoidance responses and decrease in escape responses over the session. The number of escape losses (failing to make a response during the full shock delivery period), which is recorded, could reflect an absence of learned association between cue and shock or an increase in freezing behavior with fear. Active avoidance can be evaluated using various test paradigms, for example, one-way, with actively moving from one location to another; two-way, with actively moving back and forth between two locations with appropriate cues; and three-way, with the animal's being allowed to choose between two locations to escape/avoid the stimuli such as occur in a Y-maze. As with any task requiring motor performance and latency, deficits in such ability can influence the outcome. With a fearmotivated test, the characteristics of the fear response can also influence the outcome of the test. 
Each factor can influence performance, independent of learning and memory as assessed by the testing paradigm.

\section{Exploratory Behavior/Novelty Tests: Novel Object Recognition, Social Recognition, Social Novelty}

These tests, which are based on an animal's natural propensity toward novelty, evaluate an animal's ability to recognize a previously presented stimulus or to adapt (habituate) to a novel environment. Stimuli can be an inanimate object, another animal (social recognition), or changes in the environment. When animals are exposed to a familiar object and a novel object, they frequently approach — and spend more time exploring - the novel object than the familiar object, which suggests the evidence of memory for the familiar object. In general, the paradigm consists of placing the test animal within a defined open arena and allowing it to adapt to the new environment. The animal is removed, and a novel object is placed within the arena. The animal is returned to the arena, and the number of contacts with the novel object and time spent within a defined radius of it are recorded. The animal is removed from the arena and a new object is placed into the arena in addition to the now familiar object. The proportion of time spent with each object and number of contacts are recorded over a defined period. As with any endpoint that relies on activity, deficits in motor function or activity level and exploration can influence the outcome in this paradigm. Although novel object recognition paradigms recently have been used with greater frequency in assessing the interactive nature of an animal with its environment, the range in differential preference between a familiar and a novel object is relatively narrow, seldom showing a preference above $70 \%$. Thus, these results should be interpreted with caution. Differences in the approach to a novel object between rodent strains have been demonstrated that could influence test selection.

\section{Operant Behavior}

In this form of learning, the animal displays a behavior for which they receive reinforcement. Multiple test paradigms can be used for operant conditioning, and appetitive or aversive stimuli can be used to define the reinforcement paradigm. Operant conditioning offers additional power in the ability to elicit the behavior under a schedule of required performance (schedule-controlled behavior). This feature permits increasing complexity to evaluate the nature of a learning deficit. In a more standard experimental operant paradigm, animals are placed within an operant chamber containing a bar that can be pressed or an opening to nose poke to deliver a food pellet or fluid reward; the animals then are "shaped" to perform the operant task (bar press, nose poke) on a continuous reinforcement schedule during which every appropriate behavior results in reinforcement. Once the animal is trained on the continuous reinforcement schedule-has reached a defined level of performance - the paradigm can shift to a different schedule of reinforcement. This different schedule enables examination of the animal's ability to learn a temporal association with behavior and reinforcement (interval) or an association of number of behavioral events and reinforcement (ratio), each of which can be set as fixed or variable. These then can increase in complexity or demand on the animal. Additional test paradigms and schedules can be used to test for other aspects of learning that are outside the focus of this document. Operant behavior offers a very powerful tool to assess learning capability and limitation; this test, however, is used rarely in assessments of neurotoxicity, given the demands of time and expertise and the equipment costs. 


\section{Effects of Fluoride on Learning and Memory}

\section{Evidence Synthesis}

Results were analyzed two ways. First, the collection of studies for each general type of behavioral test (Morris water maze, Y-maze, etc.) was compiled. In many cases, the overall quality of the evidence for each type of behavior test clearly would warrant a downgrade based on serious concern for risk of bias. This concern arose because several individual studies were considered to have "probably high risk of bias" in several critical domains: randomization, blinding at outcome assessment (especially when computer-assisted or automated measurements were not made), exposure characterization, and control for litter effects in developmental studies (Appendix C). Thus, these studies were excluded when reaching level-of-evidence conclusions. Almost one third of the learning and memory studies were considered to present a very serious risk of bias. Nevertheless, the results of the excluded studies are summarized below and presented in Appendix D through Appendix J. An assessment on the impact of excluding these studies found that, if included, these studies would not have appreciably altered conclusions. Quality and level-of-evidence conclusions focused on learning and memory, although studies evaluating other aspects of behavior (motor and sensory function, depression, anxiety, grooming/ urination/defecation, territorial aggression, and sexual behavior) were identified, data extracted, and assessed for risk of bias (see Appendix K through Appendix R).

No meta-analysis was conducted because few studies measured endpoints similarly in terms of study design, that is, use of same behavioral tests, dose levels, duration of exposure, lifestage at exposure, or species. Level-of-evidence conclusions were based on a narrative analysis of patterns of response across different testing paradigms and types of learning, including maze learning, avoidance learning, novel object recognition, and operant conditioning. The impact of potential confounding factors on the endpoint and subsequent interpretation also was considered. In many cases, the specificity of an effect as learning and memory could not be ascertained given the motor performance dependency of the tasks and the absence of data on motor or sensory functions to help interpret results from learning and memory tests. The extent to which skeletal fluorosis might have occurred is also unclear, especially at the higher concentrations tested; one would expect, however, that skeletal fluorosis more likely would be associated with exposure durations exceeding those that have been used in studies assessing neurobehavioral toxicity. Based on control values and the number of animals per group, most studies appear statistically underpowered to detect $<10 \%$ or $<20 \%$ change from controls for most behavioral endpoints.

Findings are summarized below with fluoride exposures expressed as ppm and as $\mathrm{mg} / \mathrm{kg}$ - $\mathrm{d}$ format in Appendix S (individual studies) and Appendix T (range across studies for a given ppm level).

\section{Exposure during Development}

A low level-of-evidence conclusion was reached based on a pattern of findings suggestive of an effect on learning and memory in rats or mice treated during gestation through postnatal day (PND) 28 or continuing until PND105 at dose levels of $\sim 11$ to $45.3 \mathrm{ppm}$ fluoride (Dong et al. 2015c; El-lethey et al. 2010; Gui et al. 2010; Jiang et al. 2014a; Wang et al. 2004; Wei et al. 
2014). ${ }^{10}$ The level-of-evidence was rated down due to concern for indirectness and lack of control for potential litter effects. Interpretation of behavioral data as a specific effect on learning and memory was hindered by concerns that the effects were secondary due to changes in motor or sensory function that could impair the animal's ability to perform the task. This was considered a form of indirectness. Additional studies are required to have higher confidence in the specificity of the responses as learning or memory impairments and to provide quantitative measures such as the effect sizes, point of departure, identification of NOEL or LOEL doses, or parameters for benchmark dose analysis. No studies evaluated a male/female difference in response to fluoride treatment (male and female offspring were pooled during analysis) and studies used Sprague Dawley or Wistar rats, so discerning any sex or species differences in response was not possible. The group size ranged between 6 and 12 for the behavioral studies. Based on variability in the control groups, these group sizes were considered statistically underpowered to detect $<10 \%$ or $<20 \%$ change from controls for most behavioral endpoints. When reported, the fluoride concentrations in the drinking water of control groups ranged from 0.15 to 1.77 ppm (Dong et al. 2015c; Gui et al. 2010; Jiang et al. 2014b; Wei et al. 2014).

\section{Morris Water Maze}

Several studies examined Morris water maze performance in animals exposed during development to 11 to 50 ppm fluoride (Dong et al. 2015c; Gui et al. 2010; Jiang et al. 2014a; Wei et al. 2014). Exposed animals often took longer or traveled farther to find the platform during the acquisition phase (referred to as "escape time") (Dong et al. 2015c; Gui et al. 2010; Jiang et al. 2014a) and magnitudes of the effects were often twice as great or more (Figure 5). Within a study, Morris water maze endpoints typically were internally consistent, that, if a slower escape time was observed at the end of the acquisition phase, the animals also displayed fewer platform crossings and spent less time in the target quadrant during the probe test. A NOEL was not identified, and the LOEL was 11.3 ppm fluoride in F1 generation Sprague Dawley male and female rats treated from gestation day (GD) 0 through PND60 (Jiang et al. 2014a). The one study that tested more than one dose level showed evidence for dose response (Jiang et al. 2014a). Some evidence was shown for dose response across studies, although variations in treatment time and endpoints assessed limited this analysis. Two studies were excluded based on concerns for overall high risk of bias (Wu et al. 2008; Zhang et al. 2009); (Appendix C). Across the remaining studies were "serious" concerns for risk of bias based on a lack of controlling for litter effects, which OHAT considered a critical risk of bias factor in developmental studies (see Figure 7). Failure to adjust for litter effects can exaggerate the statistical significance of experimental findings (Haseman et al. 2001). Also, in most of these studies, acquisition (learning) of the task either was not reported or limited to a latency measurement on the last day of training. This raised concerns for indirectness/confounding because assessment of learning and memory in the Morris water maze highly depends on latency and swimming capability. The longer latency during acquisition could reflect an effect on learning or, alternatively, impaired motor function.

Jiang et al. (2014a) exposed male and female Sprague Dawley rats to 11.3, 22.6, or 45.3 ppm fluoride beginning at 65 days of age for 10 days, at which time the animals were mated and females continued throughout pregnancy and lactation. The offspring were maintained at the

\footnotetext{
${ }^{10}$ Duplicate data from Dong et al. (2015b) appear to be reported in a second study from this group (Dong et al. 2015a).
} 
same dose level until 60 days of age. A single value for latency or total distance traveled showed increased time and distance covered to complete the task in all fluoride exposure groups. A single value of swimming speed showed no effect of fluoride exposure. Acquisition of the task over training days was not analyzed. In the memory probe test, the percentage of time spent in the target zone and the percent distance traveled in the target zone, as compared to the remaining area, were less in the fluoride-exposed rats at all concentrations tested.

Other studies used exposure paradigms designed to create fluorosis in the parental population. Gui et al. (2010) exposed a male and female parental generation of Sprague Dawley rats from 30 to 190 days of age to $\sim 30 \mathrm{ppm}$ fluoride in the drinking water. At 190 days of age, rats were mated and continued on $\sim 30 \mathrm{ppm}$ throughout lactation. The F1 offspring were maintained at the same dose level until postnatal assessment. Across 5 days of training in the Morris water maze, all animals showed acquisition (learning) of the task; across training days, however, latency remained longer for the fluoride-exposed offspring. In the memory probe trial, fluoride-exposed offspring showed less time spent in the escape quadrant of the arena and, concurrently, fewer crossings of the location of the previously hidden platform as analyzed by a paired t-test.

Dong et al. (2015b) exposed the male and female parental generation of Sprague Dawley rats from 30 to 300 days of age to 22.6 ppm fluoride in the drinking water. At 300 days of age, rats were mated and continued on $22.6 \mathrm{ppm}$ throughout lactation. The offspring were maintained at the same dose level until postnatal assessment. Latency to reach the hidden platform was longer in fluoride-exposed rats; data on acquisition (learning) of the task were not presented. In the probe trial to assess memory of the platform location, the number of times the rat crossed the previous location of the hidden platform was less in the fluoride-exposed rats. Similar changes were observed in the parental population. Apparently, identical data were reported in a second study from this group (Dong et al. 2015a).

Wei et al. (2014) established fluorosis in 30-day-old male and female Sprague Dawley rats by drinking water exposure to $22.6 \mathrm{ppm}$ fluoride for 150 days. Rats were mated, and exposure continued until PND30. The fluoride-treated offspring took longer to find the hidden platform in the Morris water maze probe test.

\section{Classic Maze}

Acquisition but impaired performance in the classical maze test was reported in Wistar rats exposed to 45.3 ppm fluoride via drinking water from GD8 to PND105 (El-lethey et al. 2010) (Figure 6, see "maze test" responses). In this study, rats were exposed to 22.6 or 45.3 ppm during GD8 to PND30 or GD8 to PND105 to assess the impact of dose and duration of exposure. The study states an overall effect of fluoride exposure on longer latency and number of errors in locating food. [Variance was not presented for each data point and significance determined by post-hoc analysis for individual days was not clearly indicated.] Performance on the first training day indicated that animals receiving $45.3 \mathrm{ppm}$ fluoride showed longer latencies and greater errors to locate feed. During the 5 days of maze testing, all fluoride-exposed rats displayed learning of the task as demonstrated by acquisition from training day 1 to day 5 and a decrease in the time required and number of errors in locating the feed at the end of the maze. Rats exposed to $45.3 \mathrm{ppm}$ from GD8 to PND105 displayed significantly longer latency to complete the maze with more errors (blind entries) compared to the control group across the entire training session. The change in performance on training day 5 as compared to training day 1 , however, suggested that choice errors no longer differed across exposure groups while latency 
remained longer and errors were more frequent only in the 45.3-ppm group exposed at PND105. No effects were reported for the 22.6-ppm dose group exposed until PND30. Duration of exposure was a significant influence on latency only to complete the maze test. [Based on text in the results and discussion, the authors do not appear to have considered effects in the 22.6-ppm dose groups or the 45.3-ppm GD8 to PND30 group significant.] One other study evaluating maze test performance was excluded based on concerns for overall high risk of bias (Băran-Poesina et al. 2013) (Appendix C).

\section{Passive Avoidance}

Wang et al. (2004) treated 30-day-old Wistar rats with fluoride (controls <0.6 ppm; fluoride $43.5 \mathrm{ppm}$ ) in the drinking water for 3 months, which were then impregnated and continued on fluoride in the drinking water throughout lactation. Offspring were continued on fluoride for a minimum of 30 days post-weaning. Animals were maintained on a dry food diet containing $25.57 \mathrm{mg} / \mathrm{kg}$ fluoride. Learning and memory were assessed by a "step-down" passive avoidance test at 30,60, or 90 days following cessation of fluoride. The number of errors in the initial training trial was not altered by fluoride exposure. The time to first error in test session showed 30-50\% variance, and less time was observed only in the 30-day test group (Figure 6). In the test session, at the 30-day time point only, the number of errors was greater in the fluoride-exposed group $(0.666 \pm 0.21)$ as compared to the total absence of errors in the controls. [Results for shock number are not shown in Figure 6 due to inability to calculate a percent change in number of shocks as compared to 0.] Two studies assessing passive avoidance (Bera et al. 2007; Hong et al. 2005) and two studies assessing active avoidance (Bera et al. 2007; Liu 1989) were excluded based on concerns for overall high risk of bias (Appendix C).

\section{Exploration}

Differences in exploratory activity were reported in adult rats developmentally exposed to fluoride. In this study, female Wistar rats were exposed to 22.6 or $45.3 \mathrm{ppm}$ in the drinking water during GD8 to PND30 or GD8 to PND105 to assess the impact of dose and exposure duration (El-lethey et al. 2010). All rats demonstrated habituation to the open-field arena over 3 days of 3-minute testing; percent change over time representing habituation was not calculated. Rats exposed to $43.5 \mathrm{ppm}$ fluoride via drinking water showed higher activity levels and an increase in the number of freezing instances (time spent not moving). Exploratory activity in an arena allowing for head exploration into holes in the floor (head dipping) showed no significant difference in the change in performance from day 1 to day 2 between dose groups (Figure 6). Across the 2 days, rats exposed to 43.5 ppm until PND150 showed a higher level of exploratory activity measured by rearing and head-dipping as compared to controls. Variance was not presented for each data point, and significance determined by post-hoc analysis at individual days was not indicated. No effects were observed in animals exposed to $22.6 \mathrm{ppm}$ fluoride. [Based on text in the results and discussion, the authors do not appear to have considered effects in the 22.6-ppm dose groups or the 45.3-ppm GD8 to PND30 group significant.] Two studies were excluded based on concerns for overall high risk of bias (Bera et al. 2007; Niu et al. 2014) (Appendix C).

\section{Findings from High Risk of Bias Studies}

Nine developmental studies were excluded based on concerns for overall high risk of bias (Table 7, see also Appendix D through Appendix J). Of these, three developmental studies were identified that tested concentrations of fluoride at 10 ppm or lower (Bera et al. 2007; Wu et al. 
2008; Zhang et al. 2009). In the Bera et al. (2007) study, Harlan Wistar female rats received daily gavage doses of fluoride ( 7 and 14 ppm) from GD1 throughout lactation to PND9. Control rats received deionized water. Fluoride-exposed offspring showed no differences in multiple endpoints assessing open-field activity. Males showed decreased performance on the roto-rod, passive avoidance, and active avoidance with significance observed at $14 \mathrm{ppm}$. All animals showed a normal habituation to a novel object. The authors state that female rats receiving $14 \mathrm{ppm}$ showed no shift from trial 1 to trial 2 of the initial novel object exploration test or the preference novel object test; however, data were not presented showing the variance of all data sets. Although the percent change in median response from trial 1 to trial 2 in the 14-ppm exposure group was less for novel object habituation, for novel object preference it was similar to that observed in controls and greater than that seen in the 7-ppm group. No effects were observed in the 7-ppm group. In Morris water maze tests, no statistically significant effects on escape latency were observed following maternal drinking water ingestion at $4.5 \mathrm{ppm}$ fluoride in ICR rats treated from GD7 to birth (Zhang et al. 2009). In Wistar rats exposed to 5 ppm fluoride from GD0 to PND45, Wu et al. (2008) reported no differences in training trials ("learning correct path") or time required to reach the platform in a water maze task. At 25 ppm fluoride, an increase was observed in latency, but variance was $>40 \%$.

Table 7. Learning and Memory Findings from Developmental Exposure Studies Excluded Based on Concern for High Overall Risk of Bias

\begin{tabular}{|c|c|c|c|}
\hline Reference & Treatment & NOEL & LOEL \\
\hline $\begin{array}{l}\text { 1. Băran-Poesina et } \\
\text { al. (2013) }\end{array}$ & $\begin{array}{l}\text { NMRI mice }(\mathrm{F} 1, \mathrm{n}=\text { not } \\
\text { reported, } \partial^{\wedge}+\text { ); drinking water } \\
\text { during development with } 18.5 \\
\text { or } 37.1 \mathrm{ppm}(5 \text { or } 10 \mathrm{mg} / \mathrm{kg}-\mathrm{d}) \\
{[\mathrm{P} 0 \text { generation from GD } 8 \text { to }} \\
\text { PND0; F1 dosing duration } \\
\text { unclear] }\end{array}$ & - & $\begin{array}{l}18.5 \mathrm{ppm}(5 \mathrm{mg} / \mathrm{kg}-\mathrm{d}): \underline{\text { maze test on }} \\
\text { PND21: } \uparrow \text { completion time in }{ }^{\uparrow}+\end{array}$ \\
\hline 2. Basha et al. (2011) & $\begin{array}{l}\text { Wistar rat }(\mathrm{F} 1-\mathrm{F} 3, \mathrm{n}=\text { not } \\
\text { reported, } 3 \text { O }) \text {; drinking water } \\
\text { during development with } 100 \text { or } \\
200 \mathrm{ppm}(16 \text { or } 32 \mathrm{mg} / \mathrm{kg}-\mathrm{d}) \\
\text { [GD0 to lactation, exposure } \\
\text { throughout life in } \mathrm{F} 1 \text { and } \mathrm{F} 2 \\
\text { generations] }\end{array}$ & - & $\begin{array}{l}100 \mathrm{ppm}(16 \mathrm{mg} / \mathrm{kg}-\mathrm{d}): \\
\text { T-maze on PND30: } \uparrow \text { errors in } \\
\text { retention phase and } \uparrow \text { time to } \\
\text { reach learning standard (F1-F3 } \\
\text { generation) }\end{array}$ \\
\hline 3. Bera et al. (2007) & $\begin{array}{l}\text { Wistar rat }\left(\mathrm{F} 1,6-12 \mathrm{~J}^{\lambda}+\right) ; \\
\text { gavage to dam with } 7 \text { or } 14 \mathrm{ppm} \\
(1.13 \text { or } 2.26 \mathrm{mg} / \mathrm{kg}-\mathrm{d})[\mathrm{GD} 0 \text { to } \\
\text { PND9] }\end{array}$ & $\begin{array}{l}7 \mathrm{ppm} \\
(1.13 \mathrm{mg} / \mathrm{kg}-\mathrm{d})\end{array}$ & $\begin{array}{l}14 \mathrm{ppm}(2.26 \mathrm{mg} / \mathrm{kg}-\mathrm{d}) \text { : active } \\
\text { avoidance on PND60: } \downarrow \text { percentage } \\
\text { of animals displaying conditioned } \\
\text { avoidance response in } 4 \text { th session } \\
\text { (no effect in } 1 \text { st session) passive } \\
\text { avoidance on PND60: } \downarrow \text { avoidance } \\
\text { latency (no effect on approach } \\
\text { latency) } \\
\text { novel object test on PND40: } \\
\text { 个time spent exploring familiar } \\
\text { object and both objects in trial } 2\end{array}$ \\
\hline
\end{tabular}


Systematic Literature Review on the Effects of Fluoride on Learning and Memory in Animal Studies

\begin{tabular}{|c|c|c|c|}
\hline Reference & Treatment & NOEL & LOEL \\
\hline 4. Hong et al. (2005) & $\begin{array}{l}\text { Wistar rat }(\mathrm{F} 1,6 \text { ๙ै }) \text {; gavage } \\
\text { development with } 45 \mathrm{ppm} \\
(6.9 \mathrm{mg} / \mathrm{kg}-\mathrm{d})[\mathrm{GD} 0 \text { to } \\
\text { PND90] }\end{array}$ & - & $\begin{array}{l}45 \mathrm{ppm}(6.9 \mathrm{mg} / \mathrm{kg}-\mathrm{d}): \text { passive } \\
\text { avoidance on PND60-120: altered } \\
\text { response in step down test }\end{array}$ \\
\hline 5. Liu (1989) & 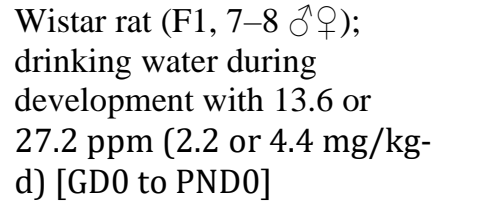 & - & $\begin{array}{l}13.6 \mathrm{ppm}(2.2 \mathrm{mg} / \mathrm{kg}-\mathrm{d}) \text { : active } \\
\text { avoidance on } \sim \text { PND } 35 \text { : } \\
\text { 个latency of conditioned avoidance } \\
\text { response on day } 9 \text { (no effect on day } \\
\text { 2) }\end{array}$ \\
\hline 6. Niu et al. (2009) & $\begin{array}{l}\text { Wistar rat }(\mathrm{F} 1, \mathrm{n}=8 \text {, sex not } \\
\text { reported); drinking water during } \\
\text { development with } 68 \mathrm{ppm} \\
(11 \mathrm{mg} / \mathrm{kg} \text {-d) [GD0 to PND21, } \\
\text { then directly dosed to } 12 \text { weeks] }\end{array}$ & - & $\begin{array}{l}68 \mathrm{ppm}(11 \mathrm{mg} / \mathrm{kg} \text {-d }) \\
\text { Y-maze at } 6 \text { or } 12 \text { weeks: } \uparrow \text { time to } \\
\text { reach learning standard at } 12 \text { weeks } \\
\text { and total reaction time at } 6 \text { weeks }\end{array}$ \\
\hline 7. Niu et al. (2014) & $\begin{array}{l}\text { Kunming mouse }(\mathrm{F} 1,15 \AA) \text {; } \\
\text { drinking water during } \\
\text { development with } 68 \mathrm{ppm} \\
(18 \mathrm{mg} / \mathrm{kg}-\mathrm{d}) \text { [GD0 to PND56] }\end{array}$ & $\begin{array}{l}68 \mathrm{ppm}(18 \mathrm{mg} / \mathrm{kg}- \\
\text { d) [no effects in } \\
\text { novel object test on } \\
\text { PND56] }\end{array}$ & - \\
\hline 8. Wu et al. (2008) & $\begin{array}{l}\text { Wistar rat }(\mathrm{F} 1,40 \stackrel{\uparrow}{\uparrow}) \text {; } \\
\text { drinking water during } \\
\text { development with } 1,5 \text { or } \\
25 \mathrm{ppm}(0.2,1 \text { or } 5.3 \mathrm{mg} / \mathrm{kg}-\mathrm{d}) \\
\text { [birth to PND45] }\end{array}$ & 5 ppm $(1 \mathrm{mg} / \mathrm{kg}-\mathrm{d})$ & $\begin{array}{l}25 \mathrm{ppm}(5.3 \mathrm{mg} / \mathrm{kg}-\mathrm{d}): \\
\text { MWM on PND } 45: \uparrow \text { : } 1 \text { numbers of } \\
\text { errors during acquisition phase and } \\
\text { 个time to find platform during probe } \\
\text { test }\end{array}$ \\
\hline 9. Zhang et al. (2009) & $\begin{array}{l}\text { ICR rat }\left(\mathrm{F} 1,9-10 \mathrm{O}^{\hat{O}}+\right) \text {; } \\
\text { drinking water during } \\
\text { development with } 1.13,2.26 \text { or } \\
4.52 \mathrm{ppm}(0.18,0.36 \text { or } \\
0.71 \mathrm{mg} / \mathrm{kg}-\mathrm{d})[\mathrm{GD} 7 \text { to PND0] }\end{array}$ & $\begin{array}{l}4.52 \mathrm{ppm} \\
(0.71 \mathrm{mg} / \mathrm{kg}-\mathrm{d}) \text { [no } \\
\text { effects in MWM } \\
\text { assessed at } 1 \text { month } \\
\text { of age] }\end{array}$ & - \\
\hline
\end{tabular}


Systematic Literature Review on the Effects of Fluoride on Learning and Memory in Animal Studies

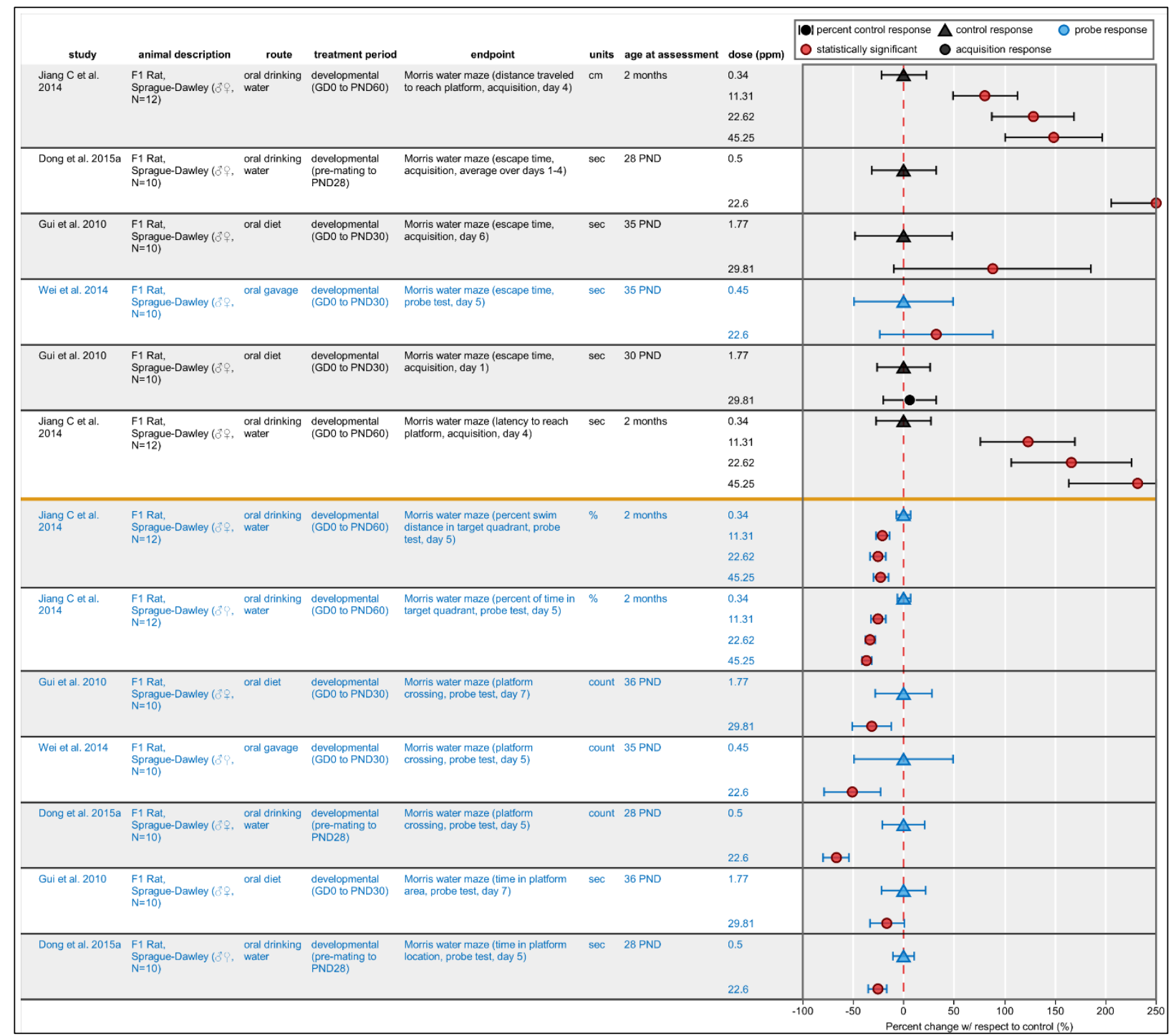

Figure 5. Results from Developmental Exposure Studies Using Morris Water Maze

Ordered by direction of expected adverse effect.

Click here for interactive graphic.

Apparently identical data were reported in Dong et al. (2015b) and Dong et al. (2015a); data only from Dong et al. (2015b) are presented in figure. Effects are grouped by a solid orange horizontal line by expected adversity direction (increase from reference/control group, then decrease from reference/control group). 


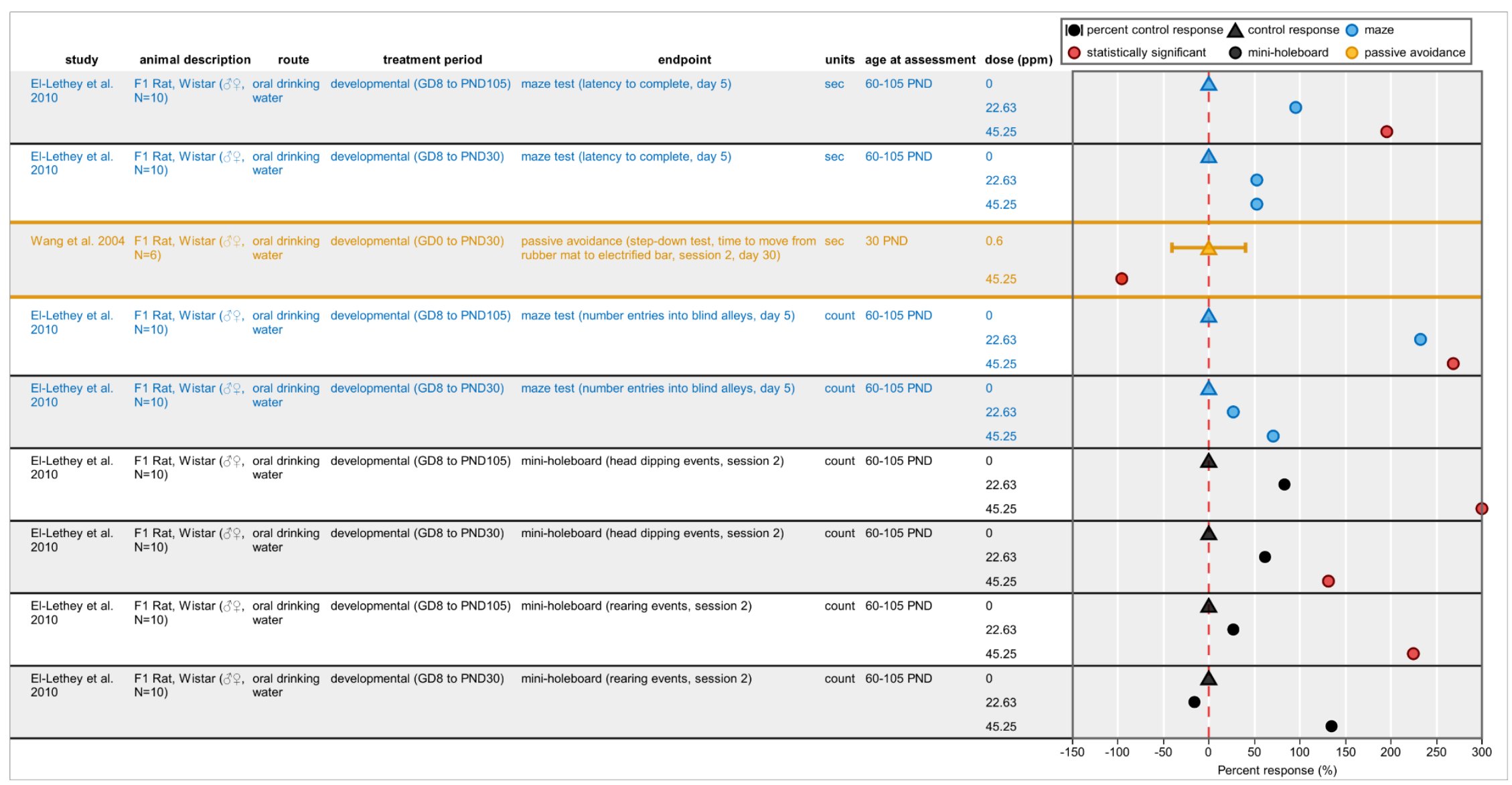

\section{Figure 6. Results from Developmental Exposure Studies in Other Maze, Exploration, and Passive Avoidance Tests}

Ordered by direction of expected adverse effect.

Click here for interactive graphic. El-lethey et al. (2010) does not report standard errors/standard deviations, so confidence intervals on percent control response cannot be calculated. Effects are grouped by a solid orange horizontal line by expected adversity direction (increase from reference/control group, then decrease from reference/control group, then any change from reference/control group). 


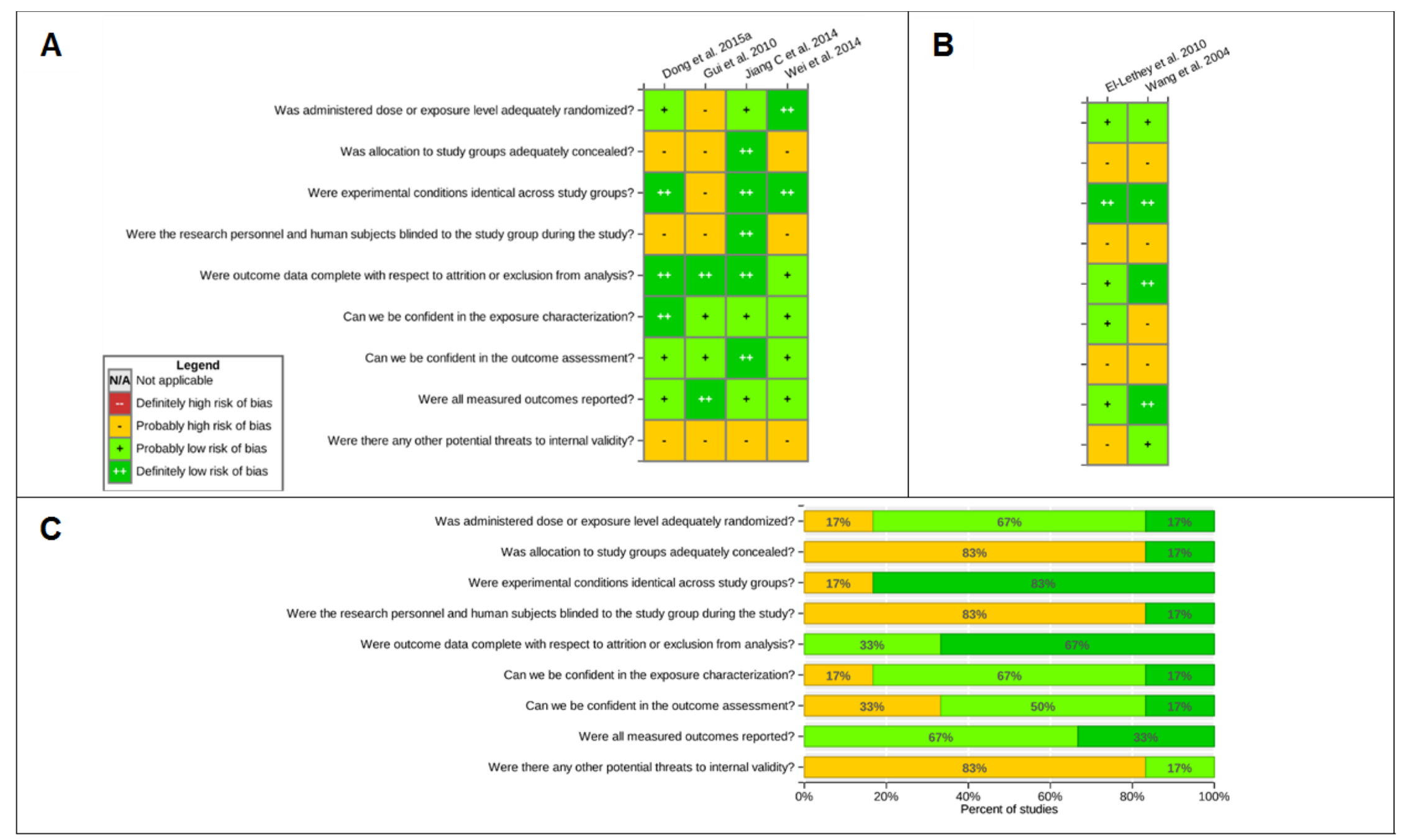

Figure 7. Risk of Bias in Studies in Studies Assessing Developmental Exposure to Fluoride

A. Risk of bias in individual studies using the Morris water maze; B. Risk of bias in individual studies assessing classic maze performance, passive avoidance or exploration; C. Risk of bias across developmental exposure studies

Apparently identical data were reported Dong et al. (2015b) and Dong et al. (2015a) [data only from Dong et al. (2015b) are presented in figure]. Studies at high overall risk of bias excluded (Băran-Poesina et al. 2013; Basha et al. 2011; Bera et al. 2007; Hong et al. 2005; Liu 1989; Niu et al. 2014; Niu et al. 2009; Wu et al. 2008; Zhang et al. 2009). 


\section{Exposure during Adulthood}

Results show a pattern of findings that suggests an effect on learning and memory when animals were exposed shortly after weaning or in adulthood at dose levels of 2.26 to $226.24 \mathrm{ppm}$ fluoride. Evidence was most abundant and strongest based on findings from the Morris water maze with effects reported at 2.26-62.7 ppm (Dong et al. 2015b; Dong et al. 2015c; Gao et al. 2009a; Gao et al. 2008a; Gao et al. 2008b; Gao et al. 2009b; Jiang et al. 2014b; Li et al. 2015; Liu et al. 2014; Liu et al. 2011; Liu et al. 2010; Liu et al. 2009; Zhu et al. 2012). Support for an effect on learning and memory from the Morris water maze findings was considered moderatelevel-of-evidence due to concern for indirectness. The same issues raised in developmentalexposure studies hinder interpretation of behavioral data from adult exposure, that is, secondary effects due to changes in motor or sensory function that could impair the animal's ability to perform the task. Findings from T-maze, Y-maze, passive avoidance tests, and active avoidance tests supported an effect on learning and memory at 2.71 to $100 \mathrm{ppm}$ but were considered lowlevel-of-evidence due to concerns for indirectness described above and risk of bias (lack of blinding at outcome assessment and characterization of the test compound)(Chen and Geng 2011; Chioca et al. 2008; Li et al. 2015; Niu et al. 2008; Raghu et al. 2013; Shen et al. 2004; Sun et al. 2008; Wang et al. 2006; Wu et al. 2006; Xu et al. 2001; Zhang et al. 2013; Zhang et al. 1999). Additional studies targeted to address these issues are required to achieve higher confidence in learning or memory impairments. Studies also are required that provide quantitative measures such as the effect sizes, point of departure, identification of NOEL or LOEL doses, or parameters for benchmark dose analysis. Most of the studies that indicated an effect of fluoride exposure on learning and memory were maze or avoidance studies. As in all learning tests, assessment requires measuring the acquisition of the task (increase in performance over time). When such data were available, fluoride-exposed animals often demonstrated acquisition similar to the controls. In these studies, a pattern emerged suggesting the need for (1) further evaluation of an effect on learning and memory using paradigms that are not influenced by motor functions or anxiety/fear reactions or (2) design of studies to interrogate the underlying effect influencing the performance more closely.

Most studies used rats (Sprague Dawley, Wistar); use of mouse models (BALB/c, ICR, Kunming) was more limited. No studies specifically evaluated a male/female difference in response to fluoride exposure, often only males were examined, males and females were pooled or, when sex was considered, unequal group sizes were compared. Thus, determining sex or species/strain difference in response to fluoride exposure was not clear. From the available data, the lowest concentration effects were observed in the 2.26- to 2.71-ppm range in mice (BALB/c in Morris water maze; Kunming in Y-maze) (Liu et al. 2014; Xu et al. 2001) and rats (Sprague Dawley in Morris water maze) (Liu et al. 2011; Liu et al. 2010; Liu et al. 2009). The group size used in most studies ranged between 6 and 10 animals with a limited number of studies using as few as 3 or as many as 30 . Based on variability in the control groups, these group sizes were considered statistically underpowered to detect $<10 \%$ or $<20 \%$ change from controls for most behavioral endpoints. When measured, the baseline concentration of fluoride in control drinking water ranged from 0.23 to 1 ppm (Chioca et al. 2008; Gao et al. 2009a; Gao et al. 2008b; Liu et al. 2011; Liu et al. 2010; Liu et al. 2009; Raghu et al. 2013). 


\section{Morris Water Maze}

The Morris water maze has been used to assess effects of adult exposure to 2.26-62.68 ppm fluoride (Figure 8). One study was conducted in BALB/c mice (Liu et al. 2014), while the others used Sprague Dawley rats (Dong et al. 2015c; Gao et al. 2009a; Gao et al. 2008a; Gao et al. 2008b; Gao et al. 2009b; Jiang et al. 2014b; Liu et al. 2011; Liu et al. 2010; Liu et al. 2009; Zhu et al. 2012) or Wistar rats ( $\mathrm{Li}$ et al. 2015). In general, effects observed were related to latency (time) for the animal to reach an escape platform. Within a study, Morris water maze endpoints were typically internally consistent. If a slower escape time was observed at the end of acquisition phase, the animals also displayed fewer platform crossings and spent less time in the target quadrant during the probe test. With latency at the end of training serving as a critical effect, the NOEL was 0.9 ppm fluoride (Liu et al. 2014) and the LOEL was $2.26 \mathrm{ppm}$ fluoride following 30 days of treatment in BALB/c male mice (Liu et al. 2014) and 6 months of treatment in Sprague Dawley rats in several studies from the same research team (Liu et al. 2011; Liu et al. 2010; Liu et al. 2009). None of the adult exposure Morris water maze studies were excluded based on having a high overall risk of bias and no serious concerns were present (Figure 9). In two instances, identical data appear to have been reported in separate publications ${ }^{11}$ [Dong et al. (2015a); Dong et al. (2015b) and Liu et al. (2011); Liu et al. (2009)]. For these studies, results as reported in the earliest publication are discussed below.

The one study conducted in BALB/c male mice examined effects following a 30-day exposure (4-8 weeks of age) to $0.9,2.26$, and $4.52 \mathrm{ppm}$ fluoride (Liu et al. 2014). In the pretest, no significant differences were noted in the initial swimming speed of the mice. In mice exposed to 2.26 and $4.52 \mathrm{ppm}$, acquisition was demonstrated but the shape of the acquisition curve was altered with an overall higher latency. In the probe test, mice exposed to 2.26 and $4.52 \mathrm{ppm}$ spent less time in the target escape quadrant and showed fewer crossings over the learned escape platform location. No effects were observed at $0.9 \mathrm{ppm}$ fluoride. At $4.52 \mathrm{ppm}$, authors reported clinical signs in the animals (curled up bodies, less movement, slightly wrinkled fur, and fluorosis symptoms in incisor teeth manifest as pale yellow plaques).

Ten studies assessed the effects of adult exposure in rats (primarily Sprague Dawley) lasting for 2 to 6 months at concentrations of 2.26 to $62.68 \mathrm{ppm}$ fluoride (Dong et al. 2015c; Gao et al. 2009a; Gao et al. 2008a; Gao et al. 2008b; Gao et al. 2009b; Jiang et al. 2014b; Li et al. 2015; Liu et al. 2011; Liu et al. 2010; Liu et al. 2009; Zhu et al. 2012). Seven of 11 studies appear to have represented a body of work conducted by a research team in the Department of Pathology and Molecular Biology at Guiyang Medical College (Dong et al. 2015c; Gao et al. 2009a; Gao et al. 2008b; Liu et al. 2011; Liu et al. 2009). Fluoride concentrations in the control drinking water ranged from 0.23-1 ppm (Gao et al. 2009a; Liu et al. 2011; Liu et al. 2009).

The lowest fluoride drinking water concentrations $(2.26,22.6 \mathrm{ppm})$ were examined in studies by the Guiyang Medical College team [(Gao et al. 2009a; Liu et al. 2011; Liu et al. 2010; Wu et al. 2008); 2.26 and $22.6 \mathrm{ppm}$ ] and by Zhu et al. (2012)(2.26, 4.52, and $9.05 \mathrm{ppm})$. Gao et al. (2008b) exposed male and female (pooled for analysis) Sprague Dawley rats to 2.26 or 22.6 ppm fluoride via drinking water for 3 months and reported a significantly longer escape latency at the

\footnotetext{
${ }^{11}$ Duplicate data from Dong et al. (2015a) for the P0 generation appear to be reported in Dong et al. (2015b). Only results from Dong et al. (2015a) are discussed. Some data from Liu et al. (2009) also are presented in Liu et al. (2011). Specifically, the values for escape time and acquisition on day 6 are identical, although the animal numbers per group differ. Duplicate results are summarized as part of Liu et al. (2009).
} 
end of the acquisition phase in the 22.6-ppm group. No effect was observed in platform crossings or time in target zone in the probe test in any group. Zhu et al. (2012) reported that a 30-day exposure of male and female Sprague Dawley rats (combined for the analysis) to $9.05 \mathrm{ppm}$ resulted in a longer latency to reach the hidden platform across all days of training in a Morris water maze task. Acquisition of the task was not analyzed nor was a memory probe test conducted. No effect was observed at exposure levels of 2.26 or $4.52 \mathrm{ppm}$. 
Systematic Literature Review on the Effects of Fluoride on Learning and Memory in Animal Studies

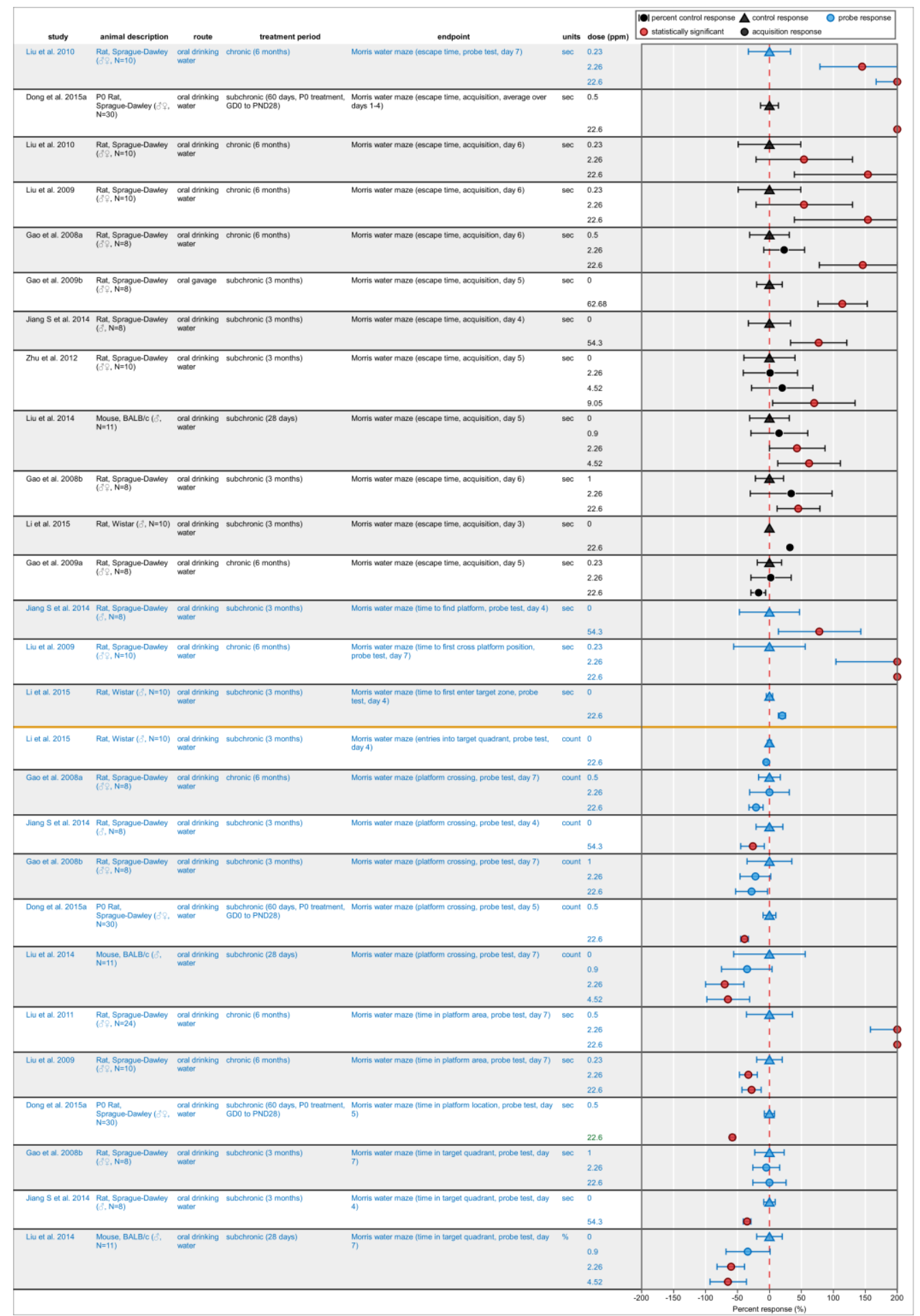

Figure 8. Results from Adult Exposure Studies Using Morris Water Maze

Ordered by direction of expected adverse effect.

Click here for interactive graphic.

Some data from Liu et al. (2009) also are presented in Liu et al. (2011) (escape time, acquisition, day 6), findings are presented under Liu et al. (2009) in figure. Apparently identical data were reported in Dong et al. (2015b) and Dong et al. (2015a); only data from Dong et al. (2015b) are presented in figure. Effects are grouped by a solid orange horizontal line by expected adversity direction (increase from reference/control group, then decrease from reference/control group). 

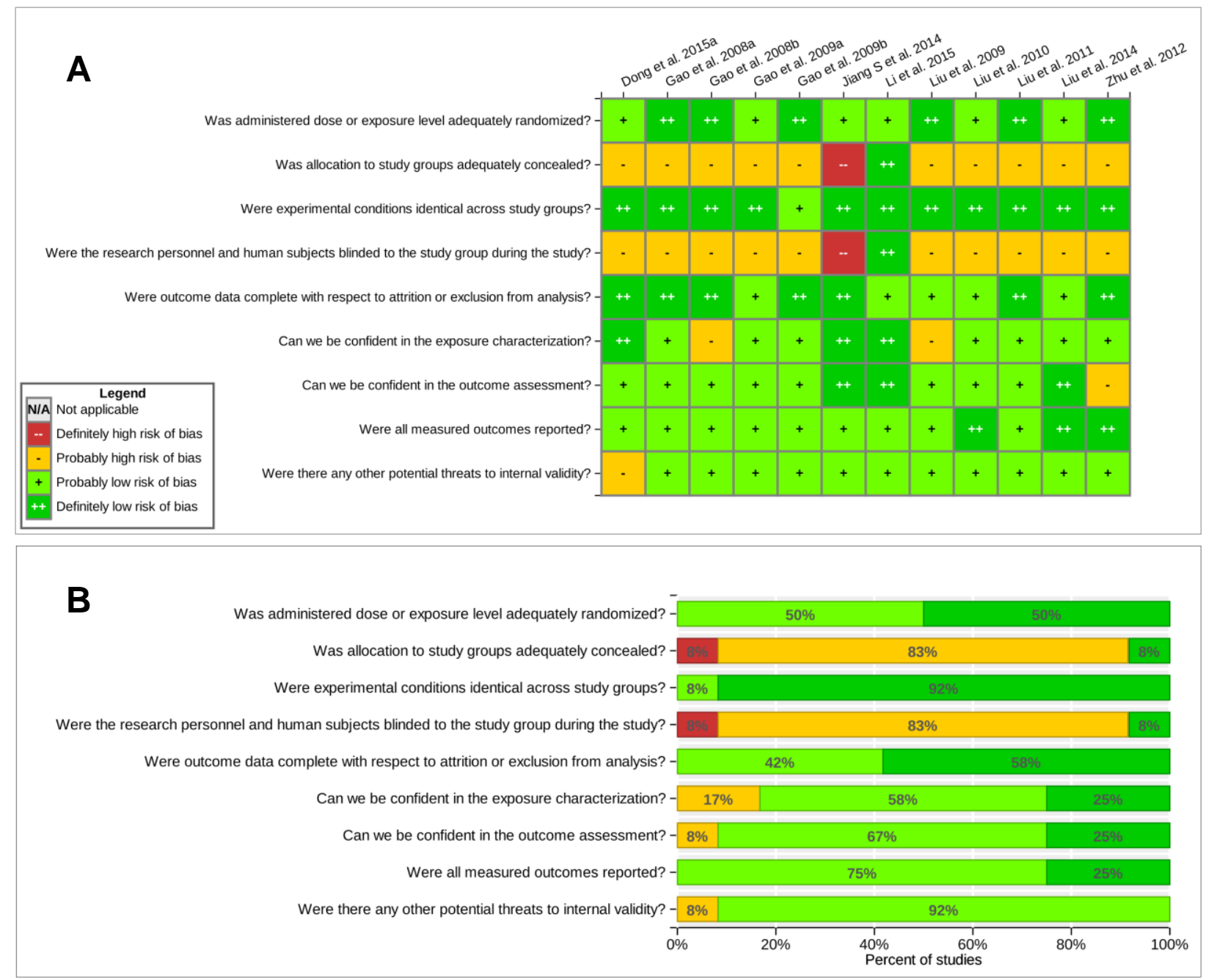

Figure 9. Risk of Bias in Studies Assessing Adult Exposure to Fluoride Using the Morris Water Maze

\section{(A) Risk of bias at the individual study level. (B) Risk of bias across studies.}

Extending the duration of exposure in Sprague Dawley rats to 6 months indicated effects on Morris water maze at $2.26 \mathrm{ppm}$ in Liu et al. (2011); Liu et al. (2010); Liu et al. (2009). Acquisition of the task was demonstrated by a progressive decrease in escape latency over the 5 days of training in all groups. A general pattern of longer latencies was reported in fluorideexposed groups as compared to controls as early as day 2 of training (Liu et al. 2009) (Figure 8). Data were analyzed each day independently and not as a progression over time. In Liu et al. (2010), the probe test suggested a higher latency for the animals to reach the location of the previously hidden platform at both dose levels (Figure 8). [Some data presented in Liu et al. (2011) appeared identical to those presented in Liu et al. (2009), and results are summarized as part of Liu et al. (2009).] Using the same dosing paradigm, other studies by the Guiyang Medical College investigators reported no significant effects on Morris water maze latency or probe test at 2.26 ppm (Gao et al. 2009a; Gao et al. 2008a; Gao et al. 2008b). In rats exposed to $22.6 \mathrm{ppm}$ fluoride, escape latency was longer at the end of Morris water maze 
training and no effect was observed on the probe test. Data on acquisition over training were not provided. In a study by Li et al. (2015), no statistically significant effects were observed on Morris water maze endpoints in Wister rats exposed to $22.6 \mathrm{ppm}$ fluoride for 3 months.

At higher fluoride concentrations (50-62.68 ppm), longer escape latency at the end of the Morris water maze training session and less preference for the hidden platform quadrant during the probe test have been reported (Dong et al. 2015c; Gao et al. 2008b; Jiang et al. 2014b). [In these studies, the fluoride-exposed group was used as a control to examine neuroprotective potential for therapeutics. Such data appear identical in Dong et al. (2015a) and Dong et al. (2015b) and, thus, are not considered as independent findings.] In Jiang et al. (2014b), the effects on escape latency, platform crossing, and time spent in target quadrant also were impaired when assessed 1 week after the acquisition phase, leading the authors to conclude that longerterm memory could be impacted.

Learning is defined as an increase in performance on a task over time and is experimentally measured in animal studies by generating an acquisition curve. Several adult Morris water maze studies did not present acquisition data, instead relying on performance at a single time point at the end of training (Gao et al. 2009a; Gao et al. 2008a; Liu et al. 2011) or averaged over the full training period (Dong et al. 2015c). This approach raises concern for data interpretation. Using terminal performance (latency or distance traveled) as an endpoint of learning - in isolation from previous trials - fails to consider differences in baseline performance that would translate to later evaluations. In the study by Gao et al. (2009a), young adult Sprague Dawley rats (male and female) received four training trials a day for 5 days. Although the four trials per day were reportedly averaged for statistical analysis, latency only on day 6 was evaluated followed by a probe trial on day 7. Escape latency on day 6 showed no difference between controls and the 2.26-ppm dose group and a significant increase was observed in the 22.6-ppm dose group. No differences across groups were observed in the probe test.

Li et al. (2015) treated 5-week-old male Wistar rats with distilled water or 22.6 ppm fluoride in the drinking water for 3 months. Rats were allowed 2 training days of 8 trials per day followed by a probe test on day 3 . Escape latency on each day and probe test performance were not statistically significant between groups. In the Morris water maze studies that did present acquisition data over 4 or 5 days, NTP calculated difference scores between the start and end of the acquisition phase to examine the progressive change in the animal's performance on the task. Effects on escape latency were more likely apparent at the end of training and either were not observed on the first day (Liu et al. 2014; Liu et al. 2010; Liu et al. 2009) or were observed but were smaller on the first day than on the last (Gao et al. 2009b; Zhu et al. 2012). With training, the differences in absolute latencies between groups could be magnified. Examining acquisition calculated as a percent change (increased performance) over time revealed that, in approximately half the studies, the change over time was equivalent to controls (Gao et al. 2009b; Jiang et al. 2014b; Liu et al. 2010; Liu et al. 2009) even though actual latency was higher. Other studies indicate impaired learning acquisition. In BALB/c mice, Liu et al. (2014) showed an approximate $45 \%$ change over time in latency in controls exposed to $0.9 \mathrm{ppm}$ fluoride for 30 days. Mice exposed to $2.26 \mathrm{ppm}$ showed an approximate $15 \%$ change while those exposed to $4.52 \mathrm{ppm}$ showed an approximate $7 \%$ change over time. In Sprague Dawley rats exposed to fluoride for 3 months, Zhu et al. (2012) reported no statistically significant change in latency over Morris water maze training sessions. An approximate 50\% change in latency was observed 
for controls and the 2.26-ppm dose group, $45 \%$ for the 4.52-ppm group, and 30\% for the 9.05ppm dose group.

\section{T- Maze and Y-Maze}

Learning can be assessed experimentally by examining behavior improvement over training sessions and determining whether a memory of the task develops that enables better performance on subsequent sessions or following an interval of time without a continuation of training trials. T-maze learning is estimated based on spontaneous alternation between arm entries. In the T-maze, adult male Wistar rats exposed to $45.3 \mathrm{ppm}$ fluoride for 30 days showed an arm bias in the spontaneous alternation paradigm and a decrease in exploratory activity (Raghu et al. 2013) (Figure 10). In the same study using a rewarded alternation paradigm, the percentage of correct responses in exposed rats decreased. The influence of the baseline arm bias on the choice of correct arm was not evaluated. In Wistar rats exposed to $22.6 \mathrm{ppm}$ fluoride for 3 months, no differences were observed in alternation rate in a Y-maze (Li et al. 2015).

Using a cued (light)/shock-reinforced Y-maze paradigm to assess learning, the number of choice errors or the number of training trials to reach a predetermined learning criterion was assessed in mice or rats exposed to $0.45-90.5$ ppm fluoride (Figure 10). Impaired performance was reported for exposure durations between 30 days and 6 months. Exposure to $2.71 \mathrm{ppm}$ for 8 weeks in mice and $22.6 \mathrm{ppm}$ for 6 months in rats resulted in a performance deficit (Figure 10) measured by number of choice errors or number of training trials to reach learning criteria (Chen and Geng 2011; Niu et al. 2008; Shen et al. 2004; Wang et al. 2006; Xu et al. 2001; Zhang et al. 2013; Zhang et al. 1999). ${ }^{12}$

In a cued Y-maze with shock reinforcement, 30-day-old ICR mice exposed to fluoride for 10 weeks showed a delay in learning (Zhang et al. 1999). At exposure levels of 4.52 ppm fluoride, mice displayed a delay in reaching a criterion of $90 \%$ correct choice rate within a session. The mice required approximately $80 \%$ more trials to achieve the criterion, as compared to controls. No effect was observed at dose levels of 0.45 and $2.26 \mathrm{ppm}$. After a 24-hour interval, mice in all groups showed a similar level of performance of $70 \%$. In another study from what appears to be the same research group, Xu et al. (2001) exposed male Kunming mice to $2.71 \mathrm{ppm}$ fluoride for 8 weeks and reported more training runs were required to meet learning criteria, but the percentage of total correct responses was unchanged (Figure 10).

At higher doses, male Wistar rats exposed to 22.6, 45.2, or 67.9 ppm fluoride for 6 months needed more Y-maze training sessions to meet learning criteria; however, the number of correct responses on the last day of training was similar to controls (Chen and Geng 2011). Shen et al. (2004) reported a similar outcome with 5 months of exposure to $67.9 \mathrm{ppm}$, with a twofold increase in the number of trials to reach criteria and 50\% decrease in percentage of correct responses in the memory trial. Niu et al. (2008) examined effects of a 30-day exposure to $67.9 \mathrm{ppm}$ fluoride in male and female (combined) Wistar rats on Y-maze performance. Training consisted of 20 trials per day until rats achieved a criterion of $90 \%$ correct choices within a session. Control rats required 3 days of training, while the 67.9-ppm group required 5 days of training. [The authors state "rats in the three treatment groups spent more days in meeting

\footnotetext{
${ }^{12}$ In several studies (Chen and Geng 2011; Shen et al. 2004; Wang et al. 2006; Xu et al. 2001), whether SD or SEM was reported is unclear. Typically, SD was assumed, based on the magnitude of the values and results of authors' statistical analysis. In Zhang et al. (2013), values were assumed to be SEMs.
} 
the learning standard of the Y-maze test" but the effect is not indicated in the figure as being statistically significant.] Rats in the 67.9-ppm group made significantly more errors at the start of training, but the number of errors showed a progressive decline over test days in both groups and by the last training day, no group differences were evident in error number or total reaction time. [Variance for error numbers on day 5 was not presented.]

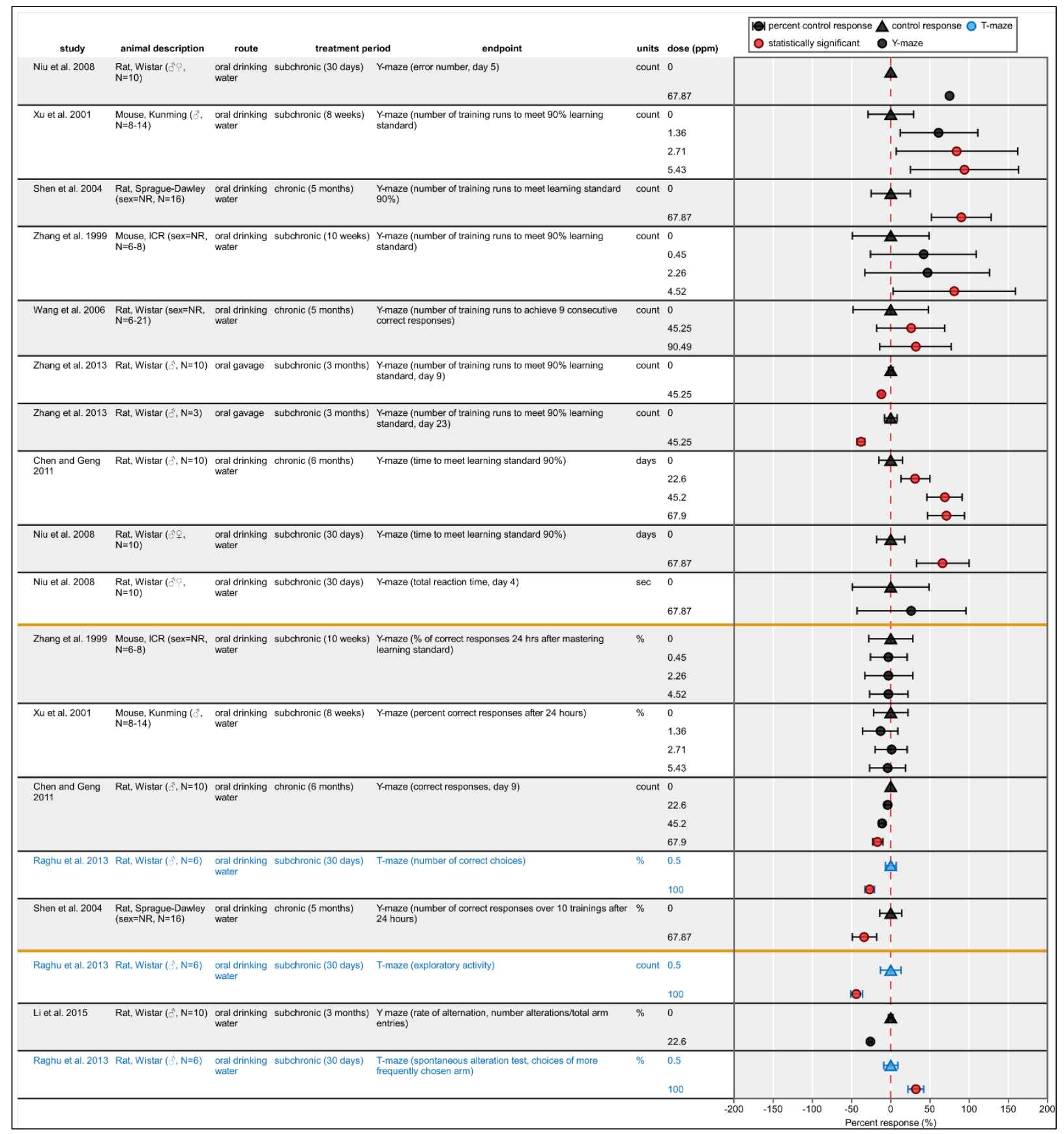

Figure 10. Results from Adult Exposure Studies Using Y- Maze and T-Maze

Ordered by direction of expected adverse effect.

Click here for interactive graphic.

Effects are grouped by a solid orange horizontal line by expected adversity direction (increase from reference/control group, then decrease from reference/control group, then any change from reference/control group). 
Wang et al. (2006) reported a longer latency in the Y-maze in Wistar rats exposed by gavage to 45.2 or 90.49 ppm fluoride for 5 months. Zhang et al. (2013) exposed male Wistar rats to daily gavage delivery of $45.2 \mathrm{ppm}$ fluoride beginning at 6 weeks of age and continuing for 3 months. The number of times the rat entered into the correct arm of the Y maze over a 20-trial session was recorded. After 9 training sessions, the retest was conducted 14 days later. All animals displayed a similar level of acquisition over the training sessions. Although not statistically analyzed by the authors, performance on the initial training day was similar. The number of times the rat entered the correct arm on the last day of training was significantly less in the fluoride-exposed rats. This difference was approximately 15\%. Memory of the task assessed 2 weeks later showed an approximately $30 \%$ difference, interpreted as lower retention in the fluoride exposed rats.

The following T-maze, Y-maze, or other maze studies were excluded from the primary analysis because of concerns for risk of bias in several critical domains (Basha and Sujitha 2012; Bhatnagar et al. 2002; Elliott 1967; Jetti et al. 2016; Zhang et al. 2001) (see Appendix C for rationale). Except for Zhang et al. (2001), the excluded studies used high concentrations of fluoride ( $>67 \mathrm{ppm}$ ). Across the remaining studies, risk of bias was a "serious" concern based on issues regarding adequate exposure characterization and lack of blinding during outcome assessment (Figure 11). Exclusion of Zhang et al. (2001) did not appreciably alter conclusions because the authors reported findings consistent with those described above for Zhang et al. (1999) and Xu et al. (2001).

\section{Passive and Active Avoidance}

In avoidance paradigms, animals are required to learn an association with an adverse event (shock) and to adjust behavior to avoid the event.

\section{Passive Avoidance}

In Raghu et al. (2013), male Wistar rats $(n=6) 30$ days of age received 100 ppm fluoride in the drinking water for 30 days. No differences were observed during the initial exploration/training phase and in the retention phase; time spent in the unsafe compartment decreased by $60 \%$ in controls and $35 \%$ in the exposed animals [details of testing paradigm not described in methods or in the cited textbook] (Figure 12). Wu et al. (2006) exposed 30-day-old male and female Wistar rats to $45.3 \mathrm{ppm}$ fluoride for up to 90 days. At 30, 60, and 90 days of exposure, rats showed no difference in errors, defined as movement from rubber mat to electrified bar, during the initial training or retention sessions; they did, however, show a shorter latency in making the first error response in the retention trial (Figure 12) shows data from 30 days only).

Two studies assessing passive avoidance were excluded from the analysis due to concerns for risk of bias (Jain et al. 2015; Jetti et al. 2016). Jetti et al. (2016) used the same model system Raghu et al. (2013) used (appear to be the same research group) and found similar results in male Wistar rats exposed to 100 ppm fluoride for 30 days. Jain et al. (2015) reported a longer retention latency in young Wistar rats (sex not reported) treated with $1.36 \mathrm{ppm}$ fluoride for 60 days beginning on PND18 [details on how or what retention latency refers to were not reported]. Across the remaining studies, concerns for risk of bias were "serious," based primarily on issues regarding adequate characterization of the exposure and lack of blinding during outcome assessment (Figure 13). 
Systematic Literature Review on the Effects of Fluoride on Learning and Memory in Animal Studies
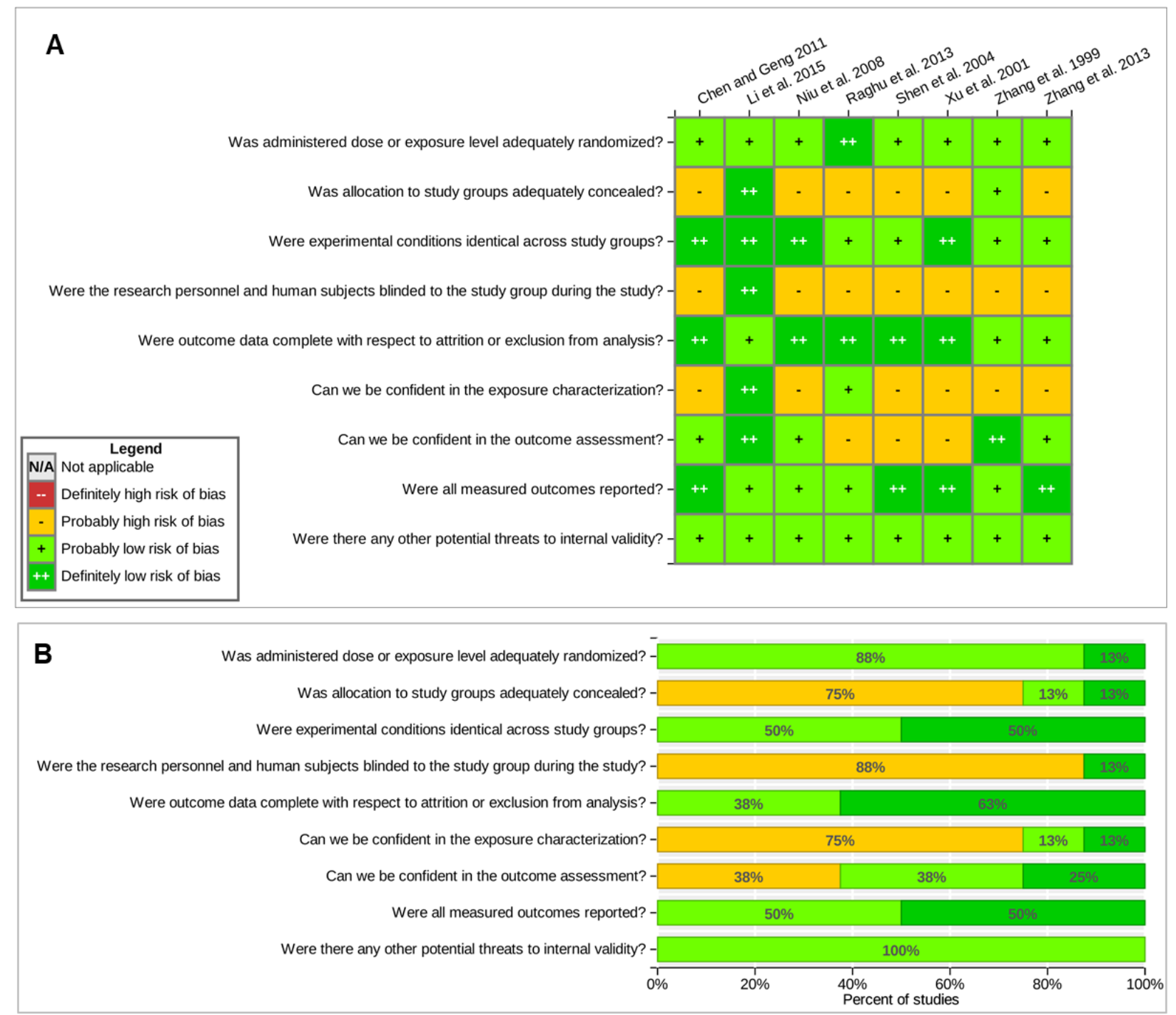

Figure 11. Risk of Bias in Studies Assessing Adult Exposure to Fluoride Using the T- or Y-Maze

(A) Risk of bias at the individual study level. (B) Risk of bias across studies. Studies at high overall risk of bias excluded (Basha and Sujitha 2012; Bhatnagar et al. 2002; Elliott 1967; Jetti et al. 2016; Zhang et al. 2001). 
Systematic Literature Review on the Effects of Fluoride on Learning and Memory in Animal Studies

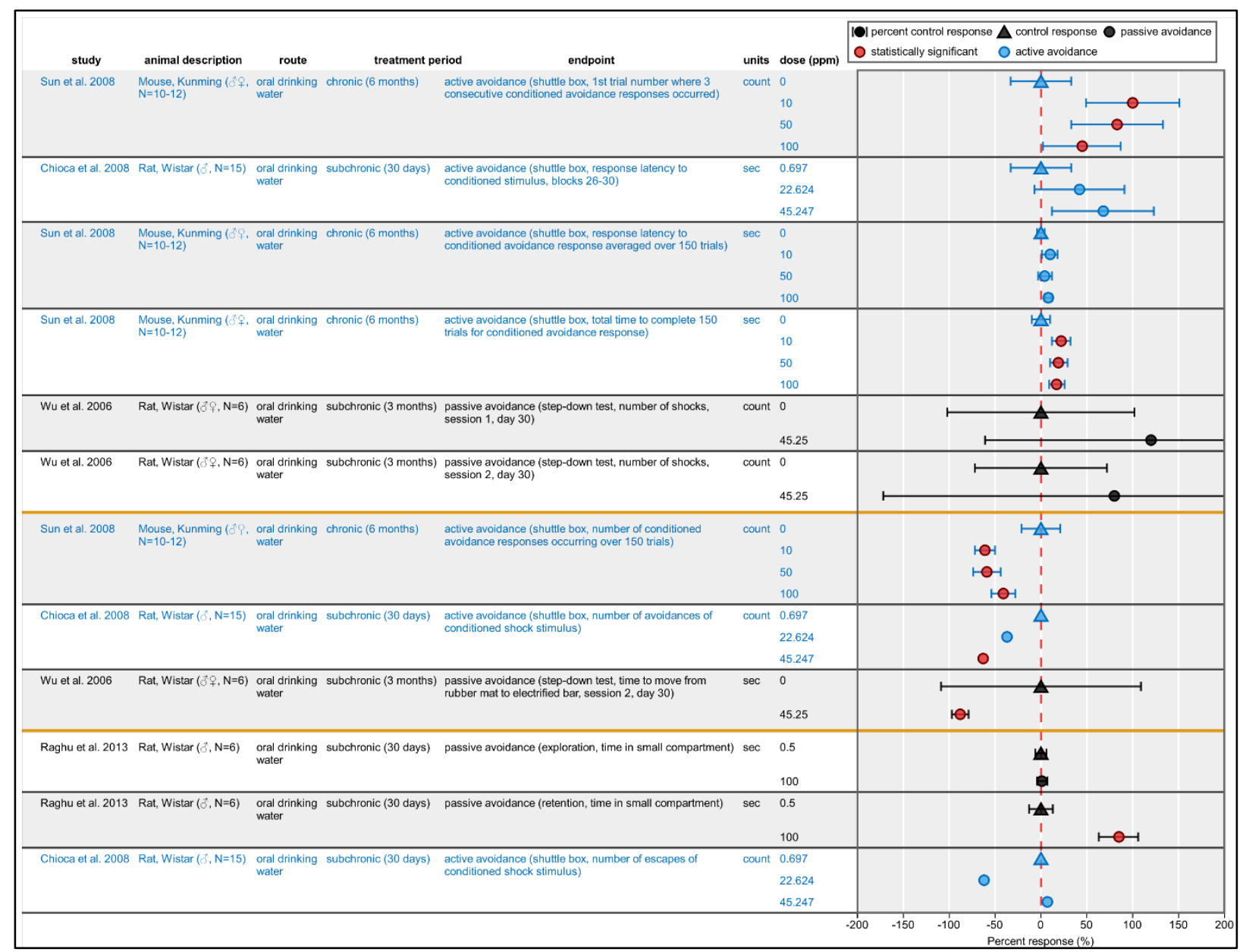

Figure 12. Results from Adult Exposure Studies Using Active and Passive Avoidance Tests

Ordered by direction of expected adverse effect.

Click here for interactive graphic.

Effects are grouped by a solid orange horizontal line by expected adversity direction (increase from reference/control group, then decrease from reference/control group, then any change from reference/control group). 
Systematic Literature Review on the Effects of Fluoride on Learning and Memory in Animal Studies

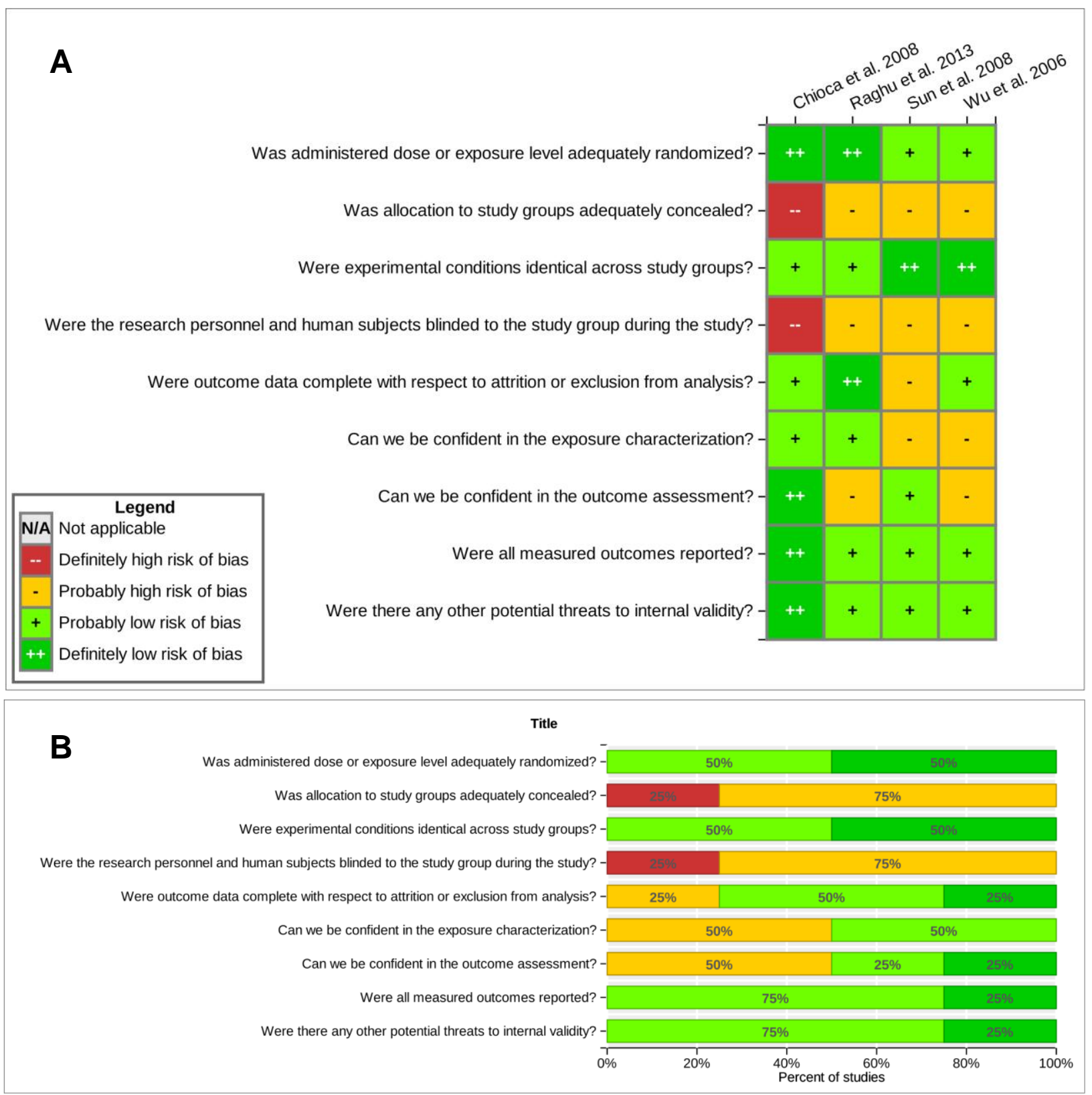

Figure 13. Risk of Bias in Adult Exposure Studies Using Active and Passive Avoidance Tests

(A) Risk of bias at the individual study level. (B) Risk of bias across studies. Studies at high overall risk of bias excluded (Jain et al. 2015; Jetti et al. 2016).

\section{Active Avoidance}

The latency to respond to a cued shock decreased over training trials in controls $(0.7 \mathrm{ppm}$ fluoride) and in adult male Wistar rats exposed to 22.6 or $45.3 \mathrm{ppm}$ fluoride for 30 days (Chioca et al. 2008). The data showed a high degree of variance. Control animals learned to shift their response pattern to avoid the shock, while a larger proportion of the fluoride-exposed animals displayed an escape, rather than avoidance, response or failed to escape the shock (Figure 12). 
In Sun et al. (2008), 60-day-old male and female Kunming mice $(\mathrm{n}=10-12)$ were exposed to 10,50 , or 100 ppm fluoride in drinking water for 6 months. Using avoidance response as a measure of learning, acquisition over five sessions was demonstrated in all exposure groups. Fluoride-exposed mice, however, took longer to complete the 150 conditioned response trials on the last training day, and all fluoride-exposed mice showed a lower level of avoidance response than controls. When data were averaged across the 150 trials, fluoride-exposed mice in all groups required more runs to display the conditioned avoidance response, took longer to perform the task, and showed a lower level of avoidance responses (Figure 12).

\section{Exploration}

Exploration in animals can reflect a learning of the environment but also can influence performance on latency or attention-dependent learning tasks. In Liu et al. (2014) 1-month old $\mathrm{BALB} / \mathrm{c}$ male mice were exposed to fluoride $(0.9,2.26$, or $4.52 \mathrm{ppm})$ via drinking water for 4 weeks. Locomotor activity and rearing were not significantly different across groups, with all groups displaying habituation to the novel arena environment over time. In a test paradigm for novel object recognition, animals showed similar exploratory activity levels and lack of preference for objects during training. When a preference of familiar versus novel was examined, total time spent with novel object was similar across groups. Preference for the novel object, however, was reduced by approximately $20 \%$ in the $2.26-$ and 4.52 -ppm groups. Han et al. (2014) exposed adult male Kunming mice to 11, 22, or 45 ppm fluoride for 180 days. No effects were observed on open-field ambulatory or rearing levels, and the time spent in different quadrants of the arena was similar across groups. In a novel object recognition paradigm, the predicted pattern for controls - to show a preference for a novel object over a familiar objectwas not observed. This pattern also was not observed in the 11- or 22-ppm fluoride-exposed group, while, in the 45-ppm group, a significant preference for the familiar object was observed. Because of the absence of the predicted control behavior, this study is not included (Figure 14). At a very high concentration of 226 ppm for 60 days, reduced exploratory motor activity was reported in male Wistar rats (Ekambaram and Paul 2003) (Figure 14). Across studies, "no serious" concerns for risk of bias were evident (Figure 15).

\section{Operant Behavior}

One study using an appetitive (food) reinforcement task to assess learning is available. Weanling female Sprague Dawley rats were exposed to fluoride in the drinking water at 33.2-155.2 ppm for 8 months. No effect of exposure was observed in rat ability to learn to press a bar to receive food pellets (Whitford et al. 2009) (Figure 14), see days to reach learning criteria and maximum response for acquisition of bar response). Rats were shifted to a fixed ratio (FR) 2 schedule of reinforcement that progressed over 5 days to a final FR-10 schedule. Rats then were placed on a differential reinforcement schedule of low rates of $20 \mathrm{sec}$ (DRL-20) that required them to pause for at least $20 \mathrm{sec}$ between bar presses to earn reinforcement. The total number of bar presses increased with increasing fixed ratios in all dose groups, which was not significantly different across dose groups. All animals displayed the ability to shift to the DRL-20 schedule with a similar level of performance demonstrated over 10 days in all dose groups. (Figure 14, see operant FR-2, FR-10, and DRL-20). Across studies "no serious" concerns for risk of bias were evident (Figure 15). 
Systematic Literature Review on the Effects of Fluoride on Learning and Memory in Animal Studies

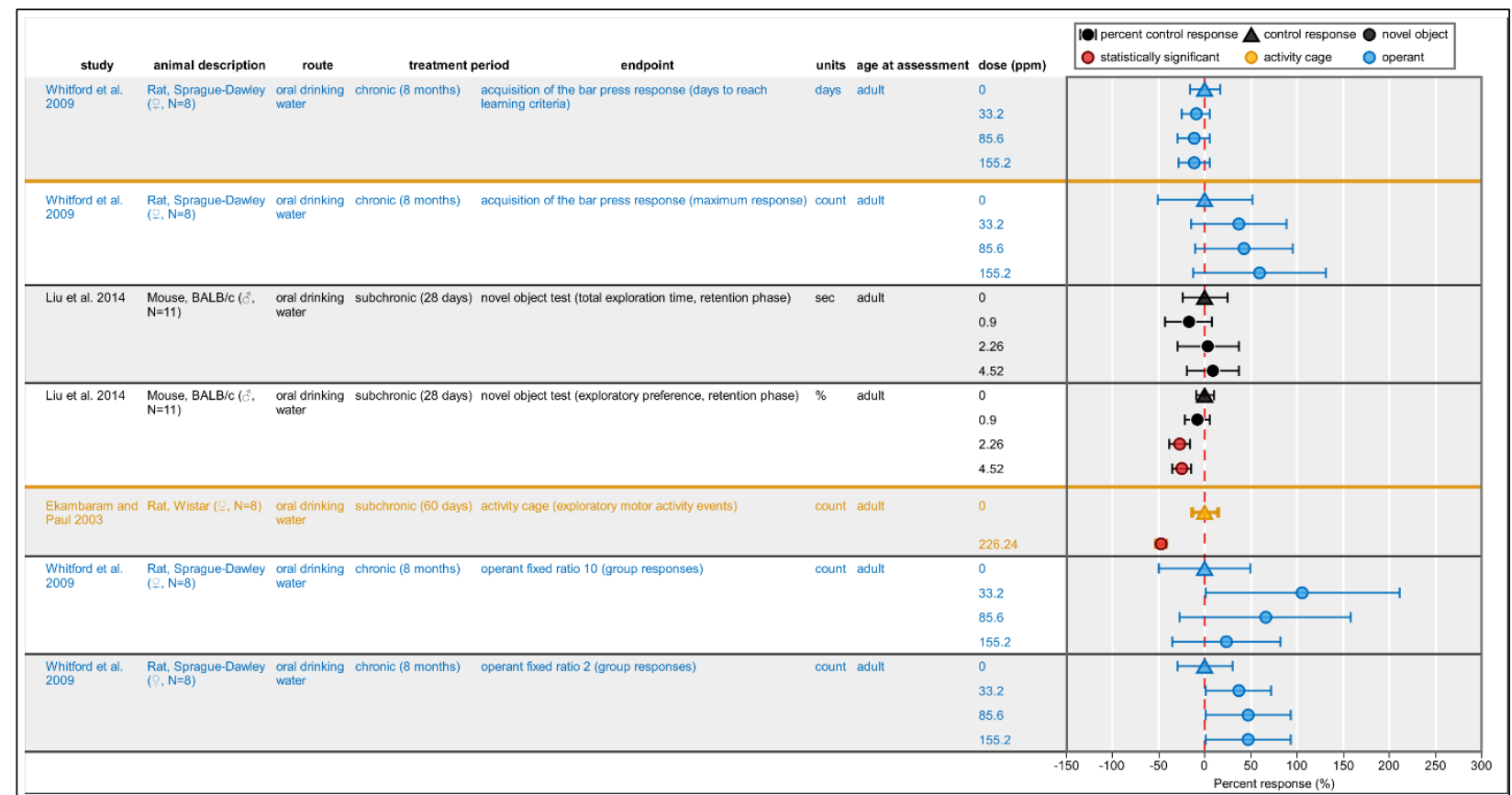

Figure 14. Results from Adult Exposure Studies Using Exploration and Operant Behavior Tests

Ordered by direction of expected adverse effect.

Click here for interactive graphic.

Effects are grouped by a solid orange horizontal line by expected adversity direction (increase from reference/control group, then decrease from reference/control group, then any change from reference/control group). 

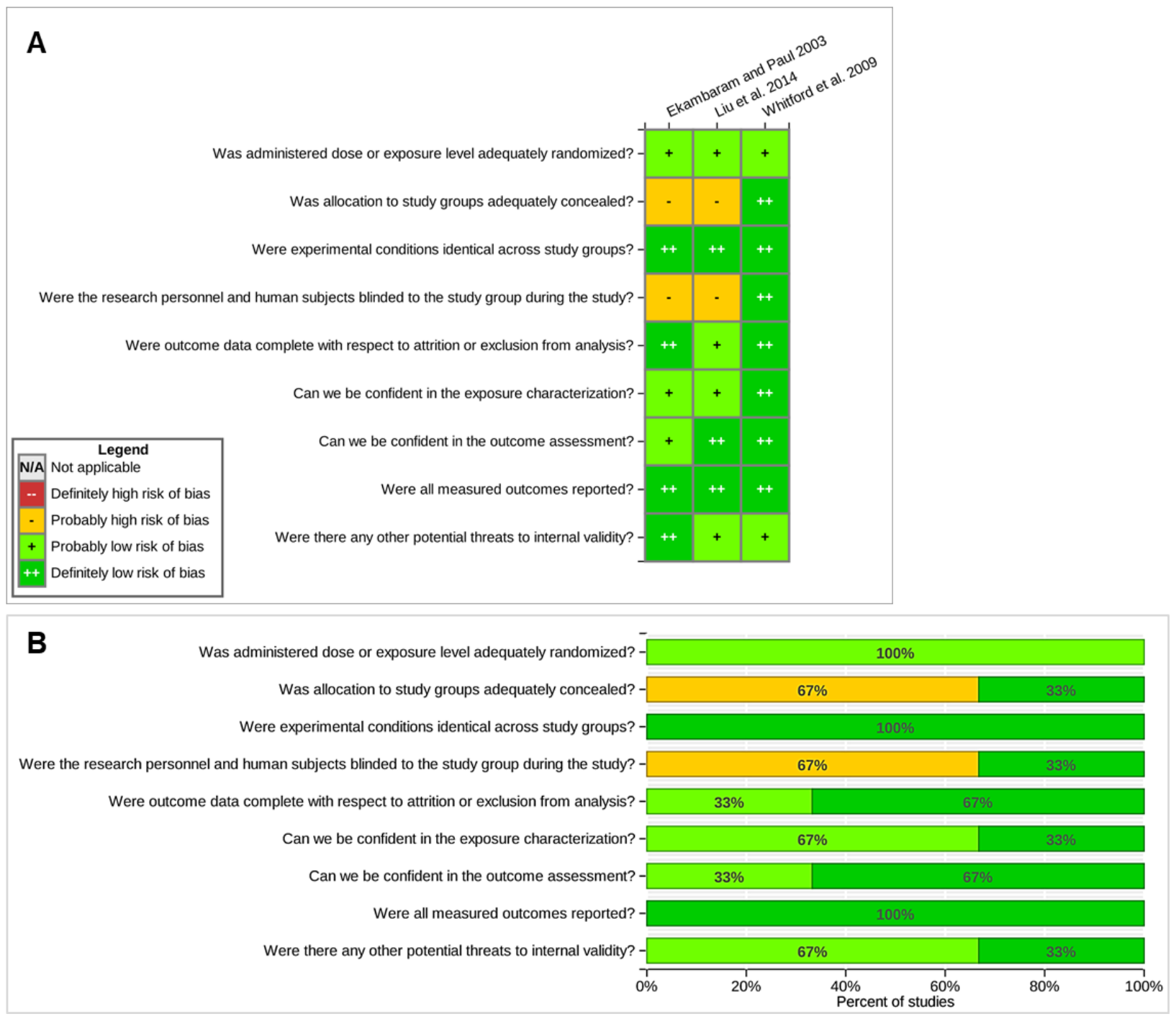

Figure 15. Risk of Bias in Adult Exposure Studies Using Exploration and Operant Behavior Tests

(A) Risk of bias at the individual study level. (B) Risk of bias across studies. Studies at high overall risk of bias excluded (Han et al. 2014).

\section{Findings from High Risk of Bias Studies}

Seven adult studies were excluded based on concerns for overall high risk of bias (Table 8). In general, these studies provided evidence to support an effect on learning and memory at high concentrations. Of these, two studies tested concentrations of fluoride at $10 \mathrm{ppm}$ or lower (Elliott 1967; Zhang et al. 2001). In Elliott (1967), no effects were observed in Hooded rats treated for 4 months with 4.24 or $42.4 \mathrm{ppm}$ in an elevated multiple T-maze. In Zhang et al. (2001), male Kunming mice exposed for 8 weeks to 2.26 or $4.52 \mathrm{ppm}$ fluoride required more training runs to reach the learning standard although percentage of correct responses was not affected 24 hours after reaching learning criteria. No effects were observed in the 0.45 -ppm group. 
Table 8. Learning and Memory Findings from Adult Exposure Studies Excluded Based on Concern for High Overall Risk of Bias

\begin{tabular}{|c|c|c|c|}
\hline Reference & Treatment & NOEL & LOEL \\
\hline $\begin{array}{l}\text { 1. Basha and Sujitha } \\
\text { (2012) }\end{array}$ & $\begin{array}{l}\text { Wistar rat (adult, } 6 \AA) \text {; } \\
\text { drinking water for } 30 \text { days with } \\
271 \mathrm{ppm}(40 \mathrm{mg} / \mathrm{kg}-\mathrm{d})\end{array}$ & - & $\begin{array}{l}271 \mathrm{ppm}(40 \mathrm{mg} / \mathrm{kg}-\mathrm{d}): \text { T-maze: } \\
\uparrow \text { time to reach learning } \\
\text { standard during retention phase } \\
\text { (but no effect on error number } \\
\text { during acquisition or retention } \\
\text { phase or time to reach standard } \\
\text { during acquisition phase) }\end{array}$ \\
\hline $\begin{array}{l}\text { 2. Bhatnagar et al. } \\
\text { (2002) }\end{array}$ & $\begin{array}{l}\text { Swiss albino mice (adult, } 5 \text { }) \text {; } \\
\text { drinking water for } 30 \text { days with } \\
14.7 \mathrm{ppm}(54.3 \mathrm{mg} / \mathrm{kg}-\mathrm{d})\end{array}$ & - & $\begin{array}{l}14 \mathrm{ppm}(54.3 \mathrm{mg} / \mathrm{kg}-\mathrm{d}): \\
\text { "simple maze" test: } \uparrow \text { latency } \\
\text { to complete }\end{array}$ \\
\hline 3. Elliott (1967) & $\begin{array}{l}\text { Hooded rat (adult, } 15 \widehat{\jmath}) \text {; } \\
\text { drinking water for } 4 \text { months } \\
\text { with } 4.24 \text { or } 42.4 \text { ppm }(0.6 \text { or } \\
6 \mathrm{mg} / \mathrm{kg}-\mathrm{d})\end{array}$ & $\begin{array}{l}42.4 \mathrm{ppm}(6 \mathrm{mg} / \mathrm{kg}-\mathrm{d})[\mathrm{no} \\
\text { effects on error number or } \\
\text { time to complete maze } \\
\text { test] }\end{array}$ & - \\
\hline 4. Han et al. (2014) & $\begin{array}{l}\left.\text { Kunming mouse (adult, } 15 \jmath^{\Uparrow}\right) \\
\text { drinking water for } 5 \text { months } \\
\text { with } 11,22 \text {, or } 45 \mathrm{ppm}(2.8 \text {, } \\
5.6 \text {, or } 11.5 \mathrm{mg} / \mathrm{kg}-\mathrm{d})\end{array}$ & 22 ppm (5.6 mg/kg-d) & $\begin{array}{l}45 \mathrm{ppm}(11.5 \mathrm{mg} / \mathrm{kg}-\mathrm{d}) \text { novel } \\
\text { object test: } \downarrow \text { discrimination } \\
\text { index (no other effects were } \\
\text { observed in novel object test) }\end{array}$ \\
\hline 5. Jain et al. (2015) & $\begin{array}{l}\text { Wistar rat (adult, } \mathrm{n}=\text { not } \\
\text { reported, sex not reported) } \\
\text { drinking water for } 60 \text { days with } \\
11.8 \mathrm{ppm}(1.8 \mathrm{mg} / \mathrm{kg}-\mathrm{d})\end{array}$ & - & $\begin{array}{l}11.8 \mathrm{ppm}(1.8 \mathrm{mg} / \mathrm{kg}-\mathrm{d}): \\
\text { passive avoidance: } \uparrow \text { latency } \\
\text { time during retention phase }\end{array}$ \\
\hline 6. Jetti et al. (2016) & $\begin{array}{l}\text { Wistar rat (adult, } 6 \widehat{\jmath}) \text {; } \\
\text { drinking water for } 4 \text { months } \\
\text { with } 100 \mathrm{ppm}(14.75 \mathrm{mg} / \mathrm{kg}-\mathrm{d})\end{array}$ & - & $\begin{array}{l}100 \mathrm{ppm}(14.75 \mathrm{mg} / \mathrm{kg}-\mathrm{d}): \underline{\mathrm{T}}- \\
\text { maze: } \downarrow \text { number correct } \\
\text { choices and exploratory } \\
\text { activity, } \uparrow \text { number of } \\
\text { spontaneous alterations. passive } \\
\text { avoidance: } \\
\uparrow \text { retention time in small } \\
\text { compartment }\end{array}$ \\
\hline $\begin{array}{l}\text { 7. Zhang et al. } \\
\text { (2001) }\end{array}$ & $\begin{array}{l}\text { Kunming mouse (adult, } 12-21 \\
\Uparrow) \text {; drinking water for } 8 \text { weeks } \\
\text { with } 0.45,2.26 \text { or } 4.52 \mathrm{ppm} \\
(0.12,0.58, \text { or } 1.16 \mathrm{mg} / \mathrm{kg}-\mathrm{d})\end{array}$ & $0.45 \mathrm{ppm}(0.12 \mathrm{mg} / \mathrm{kg}-\mathrm{d})$ & $\begin{array}{l}2.26 \mathrm{ppm}(0.58 \mathrm{mg} / \mathrm{kg}-\mathrm{d}) \\
\text { Y-maze: } \uparrow \text { number of training } \\
\text { runs to reach learning standard } \\
\text { (no effect on percentage of } \\
\text { correct responses } 24 \text { hours after } \\
\text { mastering learning standard) }\end{array}$ \\
\hline
\end{tabular}

\section{Exposure at Less Than $4 \mathrm{ppm}$}

The 2006 NRC report focused on effects in the 2- to 4-ppm range. Most fluoride neurobehavioral studies identified in the current review used higher fluoride concentrations. Findings at $<4 \mathrm{ppm}$ across all behavioral tests (learning and memory, motor function, anxiety, depression, etc.) are summarized in Figure 16 (Bartos et al. 2015; Gao et al. 2009a; Gao et al. 2008a; Gao et al. 2008b; Liu et al. 2014; Liu et al. 2011; Liu et al. 2010; Liu et al. 2009; Xu et al. 2001; Zhang et al. 1999; Zhu et al. 2012). Only one study included developmental exposure (Bartos et al. 2015). 
The lowest concentration tested was $0.9 \mathrm{ppm}$ in adult male BALB/c mice for 30 days and no effects were reported (Liu et al. 2014) for locomotor activity, tail suspension immobility time, elevated plus maze, swimming speed, or learning and memory (Morris water maze, novel object recognition). Five studies, four of which were published after the 2006 NRC review, reported learning and memory deficits in mice or rats at 2.26-2.71 ppm, including impaired performance in the Morris water maze (Liu et al. 2014; Liu et al. 2011; Liu et al. 2010; Liu et al. 2009), Ymaze (Xu et al. 2001), and novel object recognition tests (Liu et al. 2014). Liu et al. (2014) showed no alterations in swimming speed prior to Morris water maze testing.

Liu et al. (2014) also reported fewer locomotion events in an activity cage test and a longer tail suspension immobility time (taken as indicative of depression, although it can reflect decreased muscle strength) in BALB/c mice exposed to $2.26 \mathrm{ppm}$ or $4.52 \mathrm{ppm}$ for 30 days. Effects on immobility time in a forced swim test as a measure of depression were observed in the 4.52-ppm dose group. Bartos et al. (2015) did not assess learning and memory but reported delayed eye opening in F1 male and female offspring of Wistar rats treated from GD0 to PND21 with $2.26 \mathrm{ppm}$ fluoride with no differences noted in other neurodevelopmental landmarks. At 90 days of age, these offspring demonstrated lower activity levels (15 min) and an increase in entries and time spent in the open arm of an elevated plus maze. Several studies examined dose levels at $2.26 \mathrm{ppm}$ and reported no statistically significant effects on behavioral assessments (Gao et al. 2009a; Gao et al. 2008b; Zhang et al. 1999; Zhu et al. 2012). The effects on Morris water maze at $2.26 \mathrm{ppm}$ were not consistent. Several of these studies appear to have been conducted by the same research group using a very similar experimental paradigm; the authors found effects in some studies (Liu et al. 2011; Liu et al. 2010; Liu et al. 2009) but not in others (Gao et al. 2009a; Gao et al. 2008a; Gao et al. 2008b).

Risk of bias was of no serious concern among these studies (Figure 17). Reporting quality was an issue, especially regarding a lack of information on concealment during allocation of animals to study groups and blinding of research personnel during the study. Blinding at outcome assessment was not considered consistently, which represents a major concern for any endpoint obtained by observational methods. Endpoints obtained using computer-assisted equipment and measurements were of lesser concern for blinding. One of the two studies considered to have "probably high risk of bias" due to lack of blinding at outcome assessment reported effects at $2.26 \mathrm{ppm}$ (Xu et al. 2001). Information on source and purity of the fluoride was not reported consistently. Several studies that did not report this information, however, measured levels of fluoride in serum, urine, or bone and observed dose-gradients in internal exposure levels (Appendix U). 
Systematic Literature Review on the Effects of Fluoride on Learning and Memory in Animal Studies

\begin{tabular}{|c|c|c|c|c|c|c|c|}
\hline study & animed desecription & rovte $\quad$ trotetnont period & endpoint & uniss & $\begin{array}{c}\text { age out } \\
\text { assessmeent }\end{array}$ & dosen (ppm) & 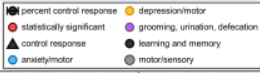 \\
\hline wenan and & 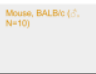 & 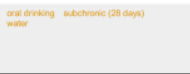 & 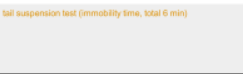 & $=$ & $\sin$ & $\begin{array}{l}0 \\
228 \\
258\end{array}$ & 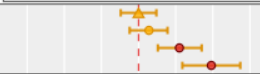 \\
\hline Wuetil : 2014 & 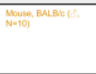 & 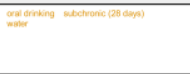 & 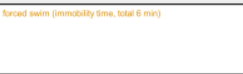 & inco & asing & 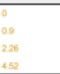 & 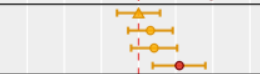 \\
\hline Lueta. . 2010 & 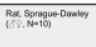 & 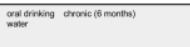 & 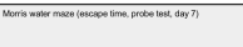 & iscect & sum & 223 & $\mapsto \overrightarrow{1}$ \\
\hline Liveral . 2010 & $\begin{array}{l}\text { Rex Sorague-Danticy } \\
(S 5, N=10)\end{array}$ & 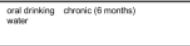 & 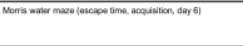 & soc & asum & 228 & $\stackrel{\overrightarrow{1}}{\longmapsto}$ \\
\hline Luental 2000 & 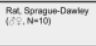 & 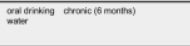 & 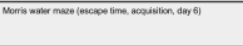 & soce & astum & 223 & $\stackrel{\Delta}{\longmapsto}$ \\
\hline 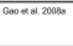 & 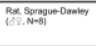 & 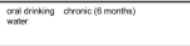 & 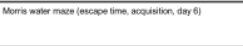 & sec & soum & $\begin{array}{l}0.5 \\
228\end{array}$ & : \\
\hline 2housal 2012 & 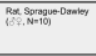 & 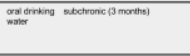 & 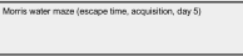 & sec & sovim & $\begin{array}{l}0 \\
228 \\
452\end{array}$ & $\stackrel{\bullet ! \bullet}{\longmapsto}$ \\
\hline Welda. 2014 & 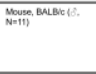 & 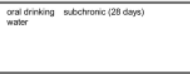 & 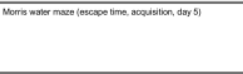 & sec & sosun & $\begin{array}{l}0 \\
09 \\
228 \\
452\end{array}$ & $\stackrel{\leftrightarrow: 0}{\longmapsto !}$ \\
\hline Gase ent. 200000 & 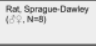 & 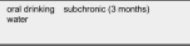 & 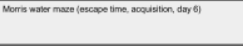 & seco & acoum & 1 & $\stackrel{41}{1}$ \\
\hline caose at al 20090 & 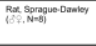 & 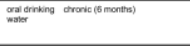 & 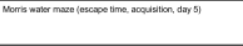 & sece & soum & 228 & $\stackrel{4}{\mapsto}$ \\
\hline Xuets.2001 & 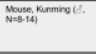 & 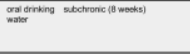 & 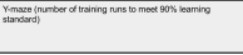 & 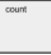 & solum & $\begin{array}{l}0 \\
136 \\
2 \pi\end{array}$ & $\stackrel{\leftrightarrow}{\bullet}$ \\
\hline 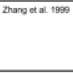 & 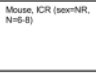 & 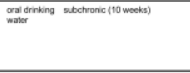 & 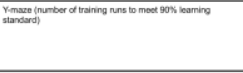 & coum & aduit & $\begin{array}{l}0 \\
0.45 \\
226 \\
452 \\
\end{array}$ & $\stackrel{\leftrightarrow: \bullet}{\longmapsto}$ \\
\hline Lweted 2009 & 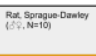 & 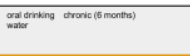 & 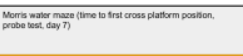 & sece & sovin & 228 & $\longmapsto$ \\
\hline Lwete. . Xo14 & 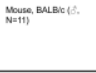 & 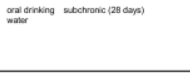 & 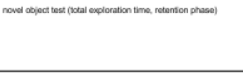 & soe & soum & $\begin{array}{l}0 \\
09 \\
226 \\
452\end{array}$ & 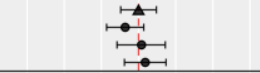 \\
\hline Luedent. 2014 & 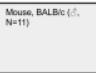 & 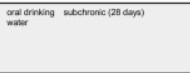 & 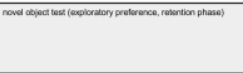 & 8 & sowit & $\begin{array}{l}0 \\
09 \\
226 \\
452\end{array}$ & 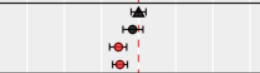 \\
\hline 2 thangedat tomo & 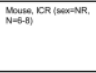 & 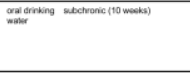 & 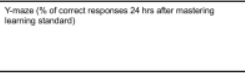 & s & aduin & $\begin{array}{l} \\
0.45 \\
228 \\
452 \\
4\end{array}$ & 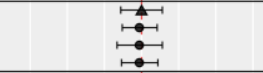 \\
\hline Xuena 20001 & 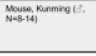 & 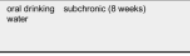 & 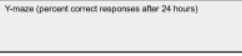 & 8 & atuil & $\begin{array}{l}0 \\
136 \\
271 \\
\end{array}$ & 能 \\
\hline CaO \& 40.20080 & 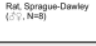 & 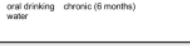 & 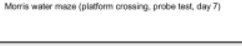 & cown & sutum & $\begin{array}{l}0.5 \\
226\end{array}$ & $\stackrel{\mapsto \Delta-1}{\bullet-1}$ \\
\hline 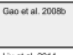 & 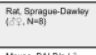 & 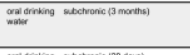 & 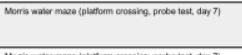 & coum & sovin & 228 & $\stackrel{\leftrightarrow}{\bullet-1}$ \\
\hline Wertal. 2014 & 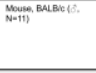 & 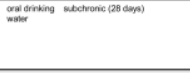 & 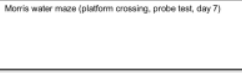 & cown & soum & $\begin{array}{l}0 \\
09 \\
226 \\
452 \\
\end{array}$ & $\begin{array}{ll}\stackrel{\bullet}{\bullet} \\
\mapsto\end{array}$ \\
\hline Wuena xot1 & 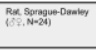 & 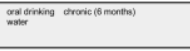 & 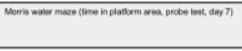 & $\ldots$ & owoun & 228 & $\mapsto$ \\
\hline Liveld. 2000 & 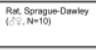 & 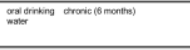 & 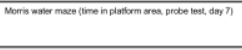 & $\sec$ & sosum & 228 & tit \\
\hline Ga0 of at 200000 & 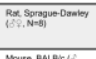 & 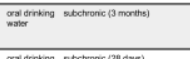 & 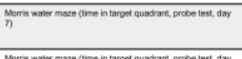 & sec & solum & 226 & 勿 \\
\hline Luets.2014 & Nouse, BNBLicis? & 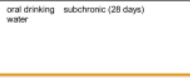 & 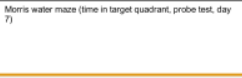 & " & sovin & $\begin{array}{l}0 \\
0.9 \\
226 \\
452\end{array}$ & 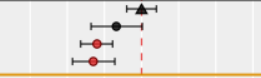 \\
\hline xietala zon & 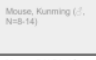 & 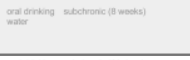 & 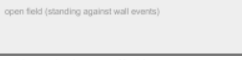 & cown & sout & $\begin{array}{l}0 \\
130 \\
211 \\
\end{array}$ & 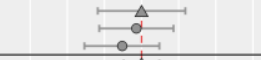 \\
\hline 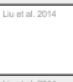 & 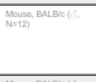 & 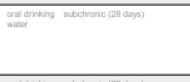 & 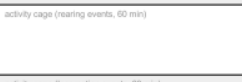 & cownt & asuit & $\begin{array}{l}0 \\
0.96 \\
225 \\
452 \\
\end{array}$ & 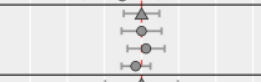 \\
\hline Wenti: one & 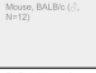 & 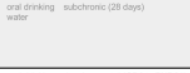 & 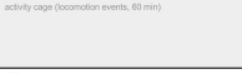 & sovent & stun & $\begin{array}{l}0 \\
09 \\
228 \\
432 \\
\end{array}$ & 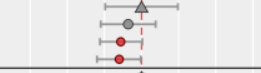 \\
\hline Berthe eat. 2015 & 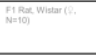 & 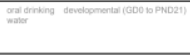 & cith trobiascesce & & 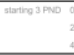 & $\begin{array}{l}0 \\
230 \\
4 a z\end{array}$ & $\begin{array}{l}\Delta \\
\vec{b} \\
0\end{array}$ \\
\hline Bumberat: ant & 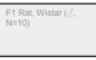 & 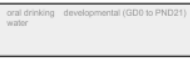 & aif moduscoo & & 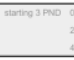 & 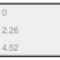 & $\begin{array}{l}1 \\
101 \\
101 \\
101\end{array}$ \\
\hline Bansosetal. 2015 & 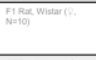 & 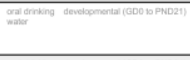 & 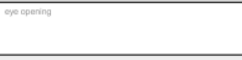 & $\operatorname{man} P \operatorname{Ric}$ & 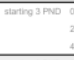 & $\begin{array}{l}0 \\
228 \\
458 \\
\end{array}$ & $\hat{\Delta}_{0}$ \\
\hline 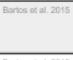 & 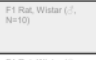 & 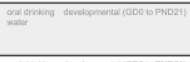 & exe coparing & $\operatorname{man} \min 0$ & matrogno & 231 & : \\
\hline $\operatorname{lons} x \in a+2015$ & 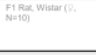 & 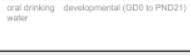 & arcosempty & $n \in m+\infty$ & 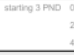 & $\begin{array}{l}0 \\
22 \pi \\
452 \\
\end{array}$ & $\frac{\Delta}{\phi}$ \\
\hline Ensise \&4. 2015 & 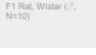 & 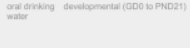 & carcopereng & $\operatorname{nan}$ PNo & thingog 3 Pete & $\begin{array}{l}228 \\
408\end{array}$ & $\dot{\Delta}$ \\
\hline
\end{tabular}


Systematic Literature Review on the Effects of Fluoride on Learning and Memory in Animal Studies

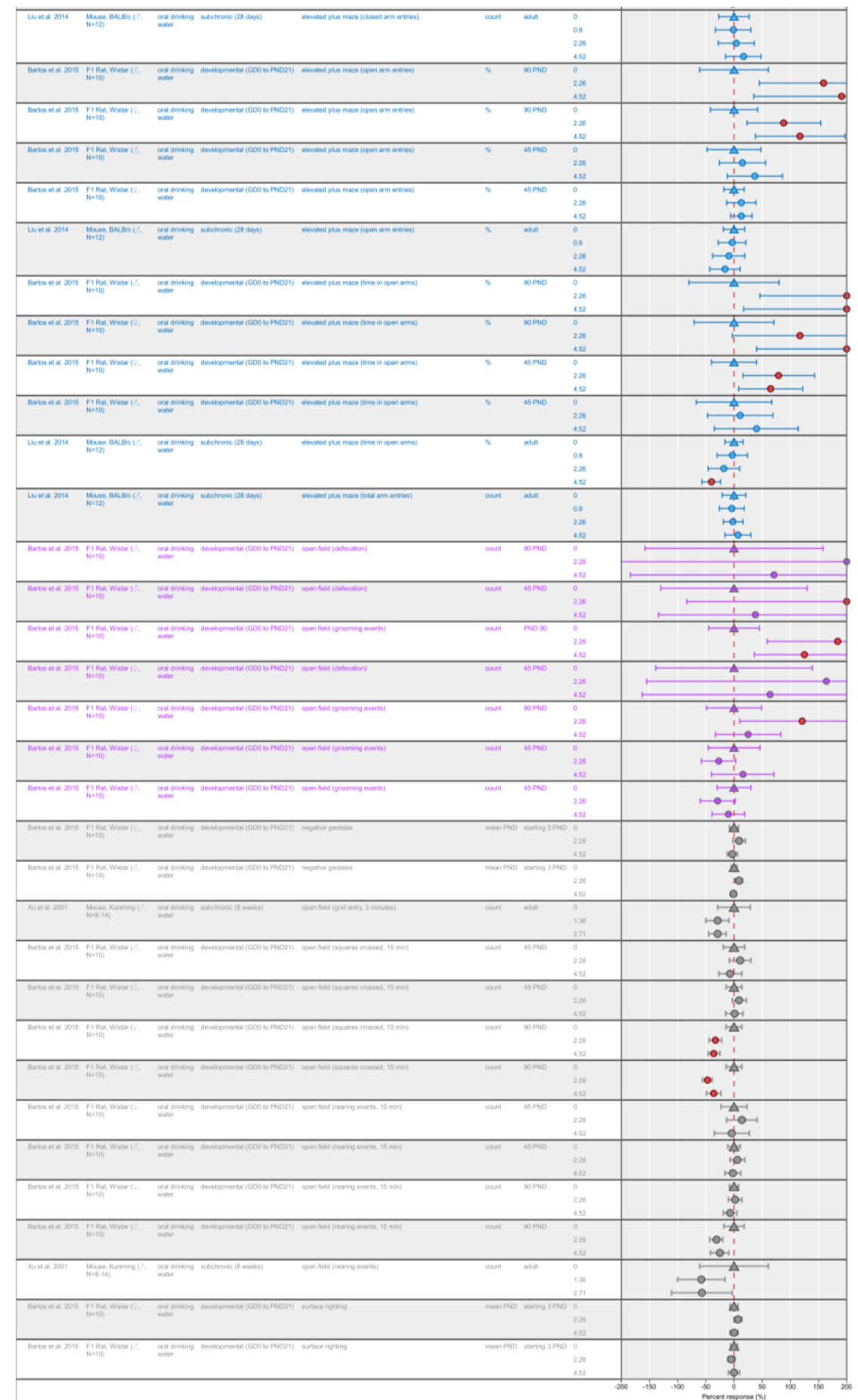

Figure 16. Results from All Studies Assessing Effects at $\leq 4 \mathrm{ppm}$

Ordered by direction of expected adverse effect.

Click here for interactive graphic.

Studies at high overall risk of bias excluded. Data not shown for Morris water maze data on acquisition day 1. Except for the Gao et al. (2009a) study, acquisition day 1 responses were not altered (escape time was significantly longer on acquisition day 1, but not on day 5). Some data from Liu et al. (2009) also are presented in Liu et al. (2011). Specifically, the values for escape time and acquisition on day 6 are identical, although the animal numbers per group differ. Duplicate results are summarized as part of Liu et al. (2009). Effects are grouped by a solid orange horizontal line by expected adversity direction (increase from reference/control group, then decrease from reference/control group, then any change from reference/control group). 

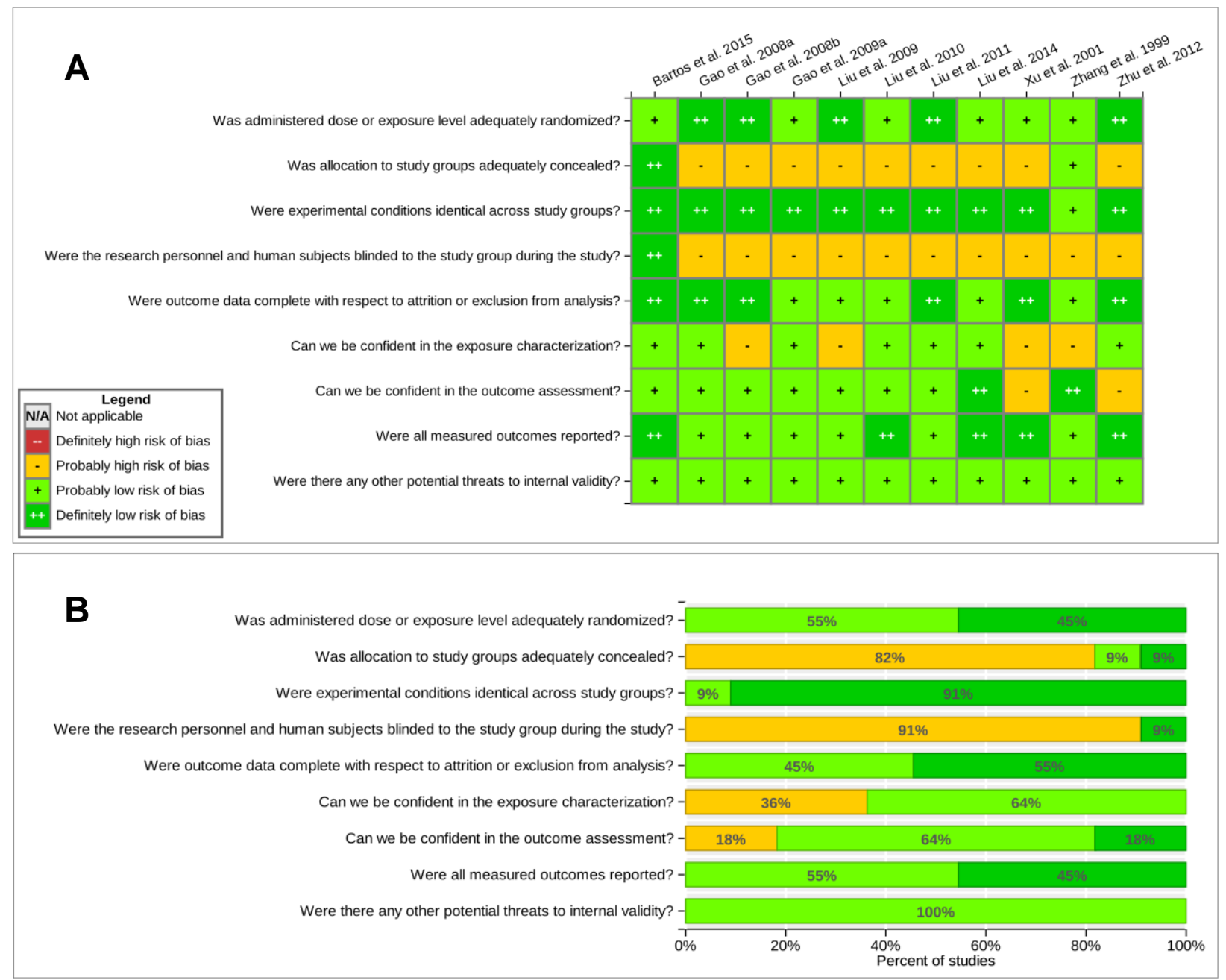

\section{Figure 17. Risk of Bias Ratings for Studies $\leq 4$ ppm}

(A) Risk of bias at the individual study level. (B) Risk of bias across studies. Studies with high risk of bias excluded (Elliott 1967; Wu et al. 2008; Zhang et al. 2009; Zhang et al. 2001).

\section{Findings from High Risk of Bias Studies (<5 ppm)}

Four studies assessing fluoride levels $<5 \mathrm{ppm}$ were excluded based on concerns for overall high risk of bias (Elliott 1967; Wu et al. 2008; Zhang et al. 2009; Zhang et al. 2001); (Table 9). Inclusion of these studies would not have appreciably influenced the conclusions described above. In general, these studies did not provide support for effects at low concentrations. Either no significant effects were observed (Elliott 1967) or effects appeared sporadically. In Zhang et al. (2009), no effects were observed in the Morris water maze at concentrations up to $4.52 \mathrm{ppm}$ in F1 male and female ICR rats of dams treated from GD7 to PND0. This study did report statistically significant decreases in locomotor activity in the open field test: Fewer grid entries occurred at 3 minutes at $2.26 \mathrm{ppm}$ (but not at 1 minute) and fewer rearing events occurred at $1.13 \mathrm{ppm}$ (but not at 2.26 or $4.52 \mathrm{ppm}$ ). Wu et al. (2008) found a change in grooming behavior (increased face scrubbing events) at $1 \mathrm{ppm}$ in the open field test in F1 male and female Wistar rats treated from GD0 to PND45 with 1, 5, or $25 \mathrm{ppm}$ fluoride. No other open-field endpoints were altered at $1 \mathrm{ppm}$, and no effects at this concentration were observed in the Morris water 
maze, tests for pain response, reflex and sensory development (surface righting, cliff avoidance, negative taxis), physical development (pinna detachment, incisor eruption,), or motor coordination.

Table 9. Findings from Studies Testing <5 ppm Fluoride Excluded Based on Concern for High Overall Risk of Bias

\begin{tabular}{|c|c|c|c|}
\hline Reference & Treatment & NOEL & LOEL \\
\hline 1. Elliott (1967) & $\begin{array}{l}\text { Hooded rat (adult, } 15 \text { ); } \\
\text { drinking water for } 4 \text { months } \\
\text { with } 4.24 \text { or } 42.4 \mathrm{ppm}(0.6 \text { or } \\
6 \mathrm{mg} / \mathrm{kg}-\mathrm{d})\end{array}$ & $\begin{array}{l}42.4 \mathrm{ppm}(6 \mathrm{mg} / \mathrm{kg}-\mathrm{d}) \\
\text { [no effects on error } \\
\text { number or time to } \\
\text { complete maze test] }\end{array}$ & - \\
\hline 2. Wu et al. (2008) & $\begin{array}{l}\text { Wistar rat }\left(\mathrm{F} 1,40 \oslash^{\top}+\right) \text {; } \\
\text { drinking water during } \\
\text { development with } 1,5 \text {, or } \\
25 \text { ppm }(0.2,1, \text { or } 5.3 \mathrm{mg} / \mathrm{kg} \text { - } \\
\text { d) [birth to PND } 45]\end{array}$ & - & $\begin{array}{l}5 \mathrm{ppm}(1 \mathrm{mg} / \mathrm{kg}-\mathrm{d}) \text { : activity: } \\
\text { 个activity time (no } \\
\text { effect at } 25 \mathrm{ppm}), \downarrow \text { number of } \\
\text { squares traveled in open field } \\
\text { test most endpoints not altered at } \\
5 \text { ppm; decreased face scrubbing } \\
\text { observed at } 1 \text { ppm in open field }\end{array}$ \\
\hline 3. Zhang et al. (2001) & $\begin{array}{l}\text { Kunming mouse (adult, } 12-21 \\
\widehat{\nwarrow}) \text {; drinking water for } 8 \text { weeks } \\
\text { with } 0.45,2.26 \text {, or } 4.52 \mathrm{ppm} \\
(0.12,0.58, \text { or } 1.16 \mathrm{mg} / \mathrm{kg}-\mathrm{d})\end{array}$ & $\begin{array}{l}0.45 \mathrm{ppm} \\
(0.12 \mathrm{mg} / \mathrm{kg}-\mathrm{d})\end{array}$ & $\begin{array}{l}2.26 \mathrm{ppm}(0.58 \mathrm{mg} / \mathrm{kg}-\mathrm{d}) \mathrm{Y}- \\
\text { maze: } \uparrow \text { number of training runs } \\
\text { to reach learning standard (no } \\
\text { effect on percentage of correct } \\
\text { responses } 24 \text { hours after } \\
\text { mastering learning standard) }\end{array}$ \\
\hline 4. Zhang et al. (2009) & 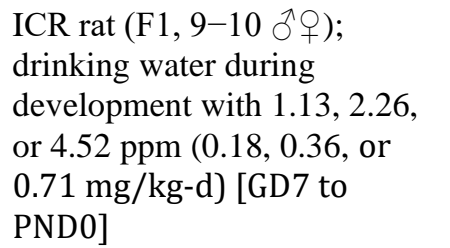 & - & $\begin{array}{l}1.13 \mathrm{ppm}(0.71 \mathrm{mg} / \mathrm{kg}-\mathrm{d}) \text { open } \\
\text { field: } \uparrow \text { number of rearing } \\
\text { events (no effect at } 2.26 \text { or } \\
4.52 \mathrm{ppm}) \text { no effects in Morris } \\
\text { water maze at any dose level }\end{array}$ \\
\hline
\end{tabular}

\section{Quality of Evidence}

Results show low-to-moderate level-of-evidence in developmental and adult exposure studies for a pattern of findings suggestive of an effect on learning and memory (Table 10). The evidence was strongest for adult exposure studies using the Morris water maze. Level-ofevidence conclusions were rated down due to concern for indirectness and risk of bias. 


\section{Discussion}

\section{Summary}

Results show low-to-moderate level-of-evidence in developmental and adult exposure studies for a pattern of findings suggestive of an effect on learning and memory (Table 10). Level-ofevidence conclusions were rated down due to concern for indirectness and risk of bias. The evidence was strongest for adult exposure studies using the Morris water maze. The evidence is strongest (moderate level-of-evidence) in animals exposed as adults tested in the Morris water maze and weaker (low level-of-evidence) in animals exposed during development.

In many cases, across the entire dose range tested, whether the effects were specifically related to learning and memory - versus a possible impact on motor or sensory function that could have impaired the ability of the animal to perform the learning and memory tests as measured-was not possible to discern. Also unclear is the extent to which skeletal fluorosis might have occurred, especially at the highest concentrations tested. Although skeletal fluorosis is associated most often with chronic durations of exposure and longer durations than those used in the neurobehavioral literature. Additional studies are required to achieve higher confidence in the specificity of the responses as learning or memory impairments and in quantitative measures such as effect sizes, points of departure, identification of NOEL or LOEL doses, or parameters for benchmark dose analysis. Based on control values (means and SD/SEs) and the number of animals per group, the studies appear statistically underpowered to detect $<10 \%$ or $<20 \%$ change from controls for most behavioral endpoints.

\section{Relevance to Human Exposure Levels}

Very few studies assessed learning and memory effects at exposure levels near 0.7 parts per million, the recommended level for community water fluoridation in the United States. No effects were observed at the lowest concentration tested of $0.9 \mathrm{ppm}(0.23 \mathrm{mg} / \mathrm{kg}-\mathrm{d})$ in adult male BALB/c mice treated with for 30 days (Liu et al. 2014). No effects were observed at this concentration in learning and memory tests (Morris water maze, novel object recognition) or in tests of motor activity, depression, or anxiety. The lowest concentration tested in the included developmental studies was 11.3 ppm (1.63 mg/kg-d) (Jiang et al. 2014a).

Several adult exposure studies, most of which were published after the 2006 NRC review of 2$4 \mathrm{ppm},{ }^{13}$ reported effects at $2.26 \mathrm{ppm}(0.33-0.59 \mathrm{mg} / \mathrm{kg}-\mathrm{d}$, depending on the study), including impaired performance in the Morris water maze (Dong et al. 2015c; Liu et al. 2014; Liu et al. 2011; Liu et al. 2010; Liu et al. 2009), Y-maze (Xu et al. 2001; Zhang et al. 1999), and novel object recognition tests (Liu et al. 2014). Other studies tested this drinking water concentration but did not report statistically significant effects (Gao et al. 2009a; Gao et al. 2008a; Gao et al. 2008b; Zhu et al. 2012). Several of the Morris water maze studies using $2.26 \mathrm{ppm}$ appear to be conducted by the same research group using a very similar experimental paradigm, finding effects at $2.26 \mathrm{ppm}$ in some studies (Liu et al. 2011; Liu et al. 2010) but not in others (Gao et al. 2009a; Gao et al. 2008b). Some studies appear to combine reporting of the duplicate data with

\footnotetext{
${ }^{13} \mathrm{At}$ levels below $4.0 \mathrm{mg} / \mathrm{L}$, NRC found no evidence substantial enough to support negative health effects other than severe dental fluorosis (NRC 2006).
} 
new results (Dong et al. 2015b; Dong et al. 2015c; Liu et al. 2011; Liu et al. 2010; Liu et al. 2009).

In the EPA exposure assessment of fluoride intake, the average drinking water concentration for public water systems at a $90 \%$ water consumption rate yielded an intake of $0.63-1.23 \mathrm{mg} / \mathrm{d}$ for children from 0.5 year to 14 years (US EPA 2010b). In the rodent literature, these daily water intakes were approximated in studies using drinking water concentrations of 7 to $9 \mathrm{ppm} .{ }^{14}$ The total oral intake of fluoride from all sources (drinking water and all other sources) was 1.2 to $2.41 \mathrm{mg} / \mathrm{d}$ for the same age groups. These total intakes were approximated by animal studies using drinking water concentrations of 9 to $29 \mathrm{ppm}$.

Assessing effects of fluoride at 0.7-ppm concentration in future studies will be difficult for various reasons. One is the background levels of fluoride in drinking water; the use of reverse osmosis filtration, however, might obviate this issue. When reported, detection level ranges in drinking water were $0.2-1.7 \mathrm{mg} / \mathrm{L}$ (Appendix V); the level of quantitation and the margin of error around those levels, however, typically were not reported. More important could be the level of fluoride in normal rodent food. Background levels in diet were less commonly reported, but included $10 \mathrm{ppm}$ (Mullenix et al. 1995) and up to $25.57 \mathrm{mg} / \mathrm{kg}$ of diet (Hong et al. 2005).

Several studies in rodents cited in this document measured serum or plasma levels of fluoride following exposures to various elevated levels in drinking water (Appendix U). These studies reported fluoride concentrations in drinking water ranging from approximately 80-8000 times higher than were measured in plasma/serum. This variability is perhaps not surprising because many factors can affect the ratios for substances that are relatively rapidly cleared from blood, including (1) the time between removal from, or the last exposure, to drinking water-which is not reported in studies; (2) the age of the animal and its related capacity of mineralized tissues to take up circulating fluoride; and (3) potential inter strain and species differences in renal clearance rates.

Understanding the relationship between "applied dose," as represented by drinking water concentration, and resulting blood levels is critical in extrapolating adverse health effects from rodent studies to humans. In this respect, the relationship between drinking water fluoride concentrations and resulting blood levels of fluoride in young healthy adult humans has been described as a numerical equivalence between drinking water concentrations expressed as $\mathrm{mg} / \mathrm{L}$,

${ }^{14} \mathrm{Using}$ a rat body weight of $0.235 \mathrm{~kg}$ and, given that $90 \mathrm{th}$ percentile human intake is $0.63 \mathrm{mg} / \mathrm{day}$ for a 1 -year-old and $1.23 \mathrm{mg} /$ day for a 14-year-old, body weight-adjusted values would be as follows for a 1-year-old and a 14-year-old:

- $\quad 0.63 \mathrm{mg} / \mathrm{day}=0.07 \mathrm{mg} / \mathrm{kg}$-d for 1 -year-old human (assumes body weight of $9 \mathrm{~kg}$ )

- $1.23 \mathrm{mg} /$ day $=0.021 \mathrm{mg} / \mathrm{kg}$-d for 14 -year-old human (assumes body weight of $60 \mathrm{~kg}$ )

Assuming that the same health effects will occur in rats when the intake is adjusted using a $\mathrm{BW}^{3 / 4}$ allometric scaling, the following body weight-adjusted intakes for rats would be:

- $0.07 \mathrm{mg} / \mathrm{kg}-\mathrm{d} \times(9 \mathrm{~kg} / 0.235 \mathrm{~kg})^{3 / 4}=1.08 \mathrm{mg} / \mathrm{kg}-\mathrm{d}$

- $\quad 0.021 \mathrm{mg} / \mathrm{kg}-\mathrm{d} \times(60 \mathrm{~kg} / 0.235 \mathrm{~kg})^{3 / 4}=1.31 \mathrm{mg} / \mathrm{kg}-\mathrm{d}$

In mg/day:

- $1.08 \mathrm{mg} / \mathrm{kg}-\mathrm{d} \times 0.235 \mathrm{~kg}=0.25 \mathrm{mg} /$ day

- $1.31 \mathrm{mg} / \mathrm{kg}-\mathrm{d} \times 0.235 \mathrm{~kg}=0.31 \mathrm{mg} / \mathrm{day}$

Converting to $\mathrm{ppm}$ would give:

- $0.25 \mathrm{mg} / \mathrm{day} / 0.034 \mathrm{~L} / \mathrm{day}=7.4 \mathrm{mg} / \mathrm{L}=7 \mathrm{ppm}$

- $0.31 \mathrm{mg} / \mathrm{day} / 0.034 \mathrm{~L} /$ day $=9.1 \mathrm{mg} / \mathrm{L}=9 \mathrm{ppm}$ 
and blood concentrations expressed as $\mu$ moles/L, or approximately a factor of 1000 (NRC 1993). ${ }^{15}$ This value falls within the range of ratios cited above for rodent studies. Dunipace et al. (1995) and National Research Council (NRC 2006), however, postulated that the applied drinking water "dose" to rats to achieve a plasma level equivalent to humans would need to be fivefold higher. Given the myriad variables that could influence these measured relationships in both animal and human studies, assuming approximate equivalence is not unreasonable, nor is assuming that health effects related to drinking water concentrations can be directly compared in rodents and humans from the perspective of kinetics.

\section{Limitations in the Literature Base}

- Deficiencies in study design, performance, and reporting limited the utility of several studies. Of the 68 included studies, 19 were considered to have a very severe risk of bias. Duplicate publication of the same results also occurred.

- Methodology descriptions of the behavioral studies often omitted specific information on how behavioral tests were conducted, configuration of the test apparatus, and specifics on test apparatus specifications, for example, for Morris water maze, the relative size of tank and platform and specifications of visual cues; size of activity arena; configuration of avoidance apparatus.

- Information was lacking on alternative sources of fluoride, for example, food or water supply.

- Relatively few developmental studies have focused on levels of fluoride exposure below $25 \mathrm{ppm}$ in drinking water (Table 6). Most of these studies have design issues including lack of reporting of randomization, blinding at outcome assessment, or lack of controlling for litter effects.

- The majority of the learning and memory studies, which indicated an effect of exposure, were maze or avoidance studies. Often, the outcome endpoint was a simple latency measurement in the final training session rather than an evaluation of the acquisition of the task (increase in performance over time) to demonstrate learning. Thus, interpretation of the data is hindered by inability to exclude alterations from baseline levels or differences in motor-related performance over the training session as contributing factors.

\section{Limitations of the Systematic Review}

- Evidence synthesis was restricted to potential learning and memory effects of fluoride.

- Additional analyses could be conducted to understand patterns of findings between learning and memory and other behavioral responses, such as motor and sensory function, and general systematic toxicity.

\footnotetext{
${ }^{15}$ On page 56-57, the text was changed from "In this respect, the relationship between drinking water fluoride concentrations and resulting blood levels of fluoride in young healthy adult humans has been described as an equivalence between drinking water concentrations expressed as $\mathrm{mg} / \mathrm{L}$, and blood concentrations expressed as $\mu \mathrm{g} / \mathrm{L}$, or approximately a factor of 1000 (NRC 1993)" to "In this respect, the relationship between drinking water fluoride concentrations and resulting blood levels of fluoride in young healthy adult humans has been described as a numerical equivalence between drinking water concentrations expressed as $\mathrm{mg} / \mathrm{L}$, and blood concentrations expressed as $\mu$ moles/L, or approximately a factor of 1000 (NRC 1993)." [August 19, 2016].
} 
- This review focused on selected behavioral measures. Studies examining fluoride exposure on endpoints assessing brain-related cellular, morphometric, or histological endpoints were considered beyond the scope of this analysis. Similarly, effects on thyroid function, which might alter specific neurobehavioral measures, were considered beyond the scope of this review. In the NRC report (NRC 2006), the histological, chemical, and molecular studies were considered to provide evidence that fluorides can interfere with the functions of the brain and the body by direct and indirect means. Potential mechanisms described included reduced brain content of lipids and phospholipids, phosphohydrolases and phospholipase D, and other proteins; inhibition of cholinesterase activity (including acetylcholinesterase); reduction in acetylcholine receptors in the brain; activation of $\mathrm{Gp}$, a protein of the $\mathrm{G}$ family, which mediates the release of many central nervous system transmitters; and increased production of free radicals in the brain. Cellular effects of the neocortext, hippocampus, and amygdala also were described in rat studies.

- Dose levels were converted to fluoride equivalents (F) expressed as $\mathrm{mg} / \mathrm{kg}$-d and ppm (e.g., $100 \mathrm{ppm}$ sodium fluoride $=45.3 \mathrm{ppm}$ fluoride). Most studies reported sodium fluoride as the form of fluoride administered. In several studies, the specific form of the chemical administered to animals was reported simply as "fluoride" with no details on counter ion, source, or purity, and no conversions to ionic fluoride equivalents were made. Dose levels expressed in fluoride equivalents would overestimate dose levels if, in fact, sodium fluoride were the test compound administered.

- Values for dose conversion (i.e., body weight, food, and water consumption) were based on EPA dosimetry guides (US EPA 1988; 1994). The European Food Safety Authority has developed more recent guidance on default values (EFSA 2012). Use of different default values can influence the dose levels as expressed in $\mathrm{mg} / \mathrm{kg}$.

- A meta-analysis was not performed in the current review, but quantitative approaches could be one way to understand sources of heterogeneity more completely and how they might affect confidence ratings, that is, heterogeneity for dose level could lead to greater confidence in dose-response assessment, while heterogeneity due to risk of bias might lead to reduced confidence.

- The ability to address potential publication bias was limited due to (1) challenges of having to select one outcome per study using approaches such as funnel plots when multiple endpoints related to the primary outcome were reported; (2) three or fewer studies being available for certain behavior tests; and (3) a lack of reporting on funding and conflict of interest in papers. In addition, analytical tools, such as funnel plots or trim-and-fill approaches, could be useful to assess publication bias but also have substantial limitations and should be interpreted with caution (Guyatt et al. 2011c). These limitations are especially pertinent for studies having small sample sizes, as is the case in the fluoride literature. In the current analysis, publication bias was described as "undetected." This determination is based on GRADE guidance to use ratings of either "undetected" or "strongly suspected," acknowledging the difficulty in having confidence that publication bias is absent and identifying a threshold to rate down the quality of evidence based on its presence (Guyatt et al. 2011c). 


\section{Data Gaps and Research Needs}

Additional studies are required to have higher confidence in the specificity of the responses as learning or memory impairments and in quantitative measures such as effect sizes, points of departure, identification of NOEL or LOEL doses, or parameters for benchmark dose analysis. Future research should consider:

- Use of low doses that are more applicable to human exposure levels and include characterization of background levels of fluoride in drinking water and diet. Assessing effects of fluoride at 0.7 -ppm concentration will be difficult, given analytical capabilities to quantify low concentrations of fluoride in drinking water and the diet. In addition, use and interpretation of results from studies that use low fluoride rodent diets is likely to be challenging because nutritional components might change as a function of methods to remove fluoride and thus, not adequately support rodent growth, development, and adult health.

- Assessment of potential confounding from effects on motor and sensory function.

- Characterization of differences in response associated with sex, lifestage at exposure, duration of exposure, species, and strain.

- Evaluation and replication of the reported neuropathology associated with exposure.

- Addressing of several common study design and reporting deficiencies in the included studies.

- In particular, additional studies should:

- Employ a wider range of doses and be appropriately powered with adequate numbers of animals to detect small effect sizes.

$\circ$ Be attentive to randomization and blinding during the study and at outcome assessment.

- Report purity and source of test compound.

- Control for litter effects in developmental studies.

- Use repeated measures statistics when appropriate, and perform separate analyses of males and females.

\section{Conclusion}

Very few studies assessed learning and memory effects in experimental animals (rats and mice) at exposure levels near 0.7 parts per million, the recommended level for community water fluoridation in the United States. At concentrations higher than 0.7 parts per million, this systematic review found a low to moderate level-of-evidence that suggests adverse effects on learning and memory in animal exposed to fluoride. The evidence is strongest (moderate levelof-evidence) in animals exposed as adults and weaker (low level-of-evidence) in animals exposed during development. Confidence in these findings was reduced primarily based on potential confounding of the learning and memory assessments by deficits in motor function or fear and risk of bias limitations. Additional research is needed, in particular to address potential effects on learning and memory following exposure during development to fluoride at levels nearer to 0.7 parts per million. NTP is conducting laboratory studies in rodents to fill data gaps 
identified by this systematic review of the animal studies. The findings from those studies will be included in a future systematic review to evaluate potential neurobehavioral effects from exposure to fluoride during development with consideration of human, experimental animal and mechanistic data. 
Systematic Literature Review on the Effects of Fluoride on Learning and Memory in Animal Studies

\section{References}

Bai J, Chen Y, Liu Q-b. 2010. Learning and memory obstacles and changes in brain growth tissue inhibitors from brick tea fluoride and alminum poisoning of rats. Chinese Journal of Control of Endemic Diseases. 25(8734):161-163.

Balaji B, Kumar EP, Kumar A. 2015. Evaluation of standardized Bacopa monniera extract in sodium fluoride-induced behavioural, biochemical, and histopathological alterations in mice. Toxicol Ind Health. 31(1):18-30. http://dx.doi.org/10.1177/0748233712468018

Balayssac D, Richard D, Authier N, Nicolay A, Jourdan D, Eschalier A, Coudore F. 2002. Absence of painful neuropathy after chronic oral fluoride intake in Sprague-Dawley and Lou/C rats. Neurosci Lett. 327(3):169-172. http://dx.doi.org/10.1016/S0304-3940(02)00421-4

Banji D, Banji OJ, Pratusha NG, Annamalai AR. 2013. Investigation on the role of Spirulina platensis in ameliorating behavioural changes, thyroid dysfunction and oxidative stress in offspring of pregnant rats exposed to fluoride. Food Chem. 140(1-2):321-331. http://dx.doi.org/10.1016/j.foodchem.2013.02.076

Băran-Poesina V, Negreș S, Dobrescu D, Dimcevici-Poesina N, Dimcevici-Poesina A, Feghiu A, Soare T, Militaru M. 2013. Experimental pharmacological researches regarding the influence of sodium fluoride in allopathic and homeopathic doses on central nervous system's performances. A correlation between behavioral response in classic maze test and morphological aspects of cerebral cortex. Farmacia. 61(4):781-799.

Bartos M, Gumilar F, Bras C, Gallegos CE, Giannuzzi L, Cancela LM, Minetti A. 2015. Neurobehavioural effects of exposure to fluoride in the earliest stages of rat development. Physiol Behav. 147:205-212. http://dx.doi.org/10.1016/j.physbeh.2015.04.044

Basha PM, Rai P, Begum S. 2011. Fluoride toxicity and status of serum thyroid hormones, brain histopathology, and learning memory in rats: a multigenerational assessment. Biol Trace Elem Res. 144(1-3):1083-1094. http://dx.doi.org/10.1007/s12011-011-9137-3

Basha PM, Sujitha NS. 2012. Combined impact of exercise and temperature in learning and memory performance of fluoride toxicated rats. Biol Trace Elem Res. 150(1-3):306-313. http://dx.doi.org/10.1007/s12011-012-9489-3

Bataineh HN, Nusier MK. 2006. Impact of 12-week ingestion of sodium fluoride on aggression, sexual behavior, and fertility in adult male rats. Fluoride. 39(4):293-301.

Bera I, Sabatini R, Auteri P, Flace P, Sisto G, Montagnani M, Potenza M, Marasciulo F, Carratu M, Coluccia A. 2007. Neurofunctional effects of developmental sodium fluoride exposure in rats. Eur Rev Med Pharmacol Sci. 11(4):211.

Bhatnagar M, Rao P, Jain S, Bhatnagar R. 2002. Neurotoxicity of fluoride: neurodegeneration in hippocampus of female mice. Indian J Exp Biol. 40(5):546-554.

Broadbent JM, Thomson WM, Ramrakha S, Moffitt TE, Zeng J, Foster Page LA, Poulton R. 2015. Community water fluoridation and intelligence: prospective study in New Zealand. Am J Public Health. 105(1):72-76. http://dx.doi.org/10.2105/AJPH.2013.301857 
California Office of Environmental Health Hazard Assessment (OEHHA). 2011. Meeting synopsis and slide presentations: Carcinogen identification committee meeting held on October 12, 2011. https://oehha.ca.gov/proposition-65/transcript-comment-presentation/meetingsynopsis-and-slide-presentations-carcinogen.https://oehha.ca.gov/proposition-65/transcriptcomment-presentation/meeting-synopsis-and-slide-presentations-carcinogen.

Chen H, Geng D. 2011. The establishment and assessment of animal model of chronic fluorosisinduced cognitive dysfunction in rats. Acta Acad Med Xuzhou. (5):319-322.

Chioca LR, Raupp IM, Da Cunha C, Losso EM, Andreatini R. 2008. Subchronic fluoride intake induces impairment in habituation and active avoidance tasks in rats. Eur J Pharmacol. 579(13):196-201. http://dx.doi.org/10.1016/j.ejphar.2007.10.019

Choi AL, Sun G, Zhang Y, Grandjean P. 2012. Developmental fluoride neurotoxicity: a systematic review and meta-analysis. Environ Health Perspect. 120(10):1362. http://dx.doi.org/10.1289/ehp.1104912

Crawley JN. 2007. What's wrong with my mouse?: Behavioral phenotyping of transgenic and knockout mice. 2 ed. John Wiley \& Sons.

Dabeka R, Karpinski K, McKenzie A, Bajdik C. 1986. Survey of lead, cadmium and fluoride in human milk and correlation of levels with environmental and food factors. Food Chem Toxicol. 24(9):913-921. http://dx.doi.org/10.1016/0278-6915(86)90318-2

Dong Y-T, Wang Y, Wei N, Guan Z-Z. 2015a. Expression levels of brain muscarinic acetylcholine receptor in offspring rats of drinking-water borne fluorosis. Chinese Journal of Endemiology. 34(5):326-330.

Dong Y-T, Wang Y, Wei N, Guan Z. 2015b. Expression of muscarinic acetylcholine receptors in the brain of rats with chronic fluorosis. Chinese Journal of Endemiology. 34(2):84-88.

10.3760/cma.j.issn.2095-4255.2015.02.003

Dong Y-T, Wang Y, Wei N, Zhang Q-F, Guan Z-Z. 2015c. Deficit in learning and memory of rats with chronic fluorosis correlates with the decreased expressions of M1 and M3 muscarinic acetylcholine receptors. Arch Toxicol. 89(11):1981-1991. http://dx.doi.org/10.1007/s00204-014$\underline{1408-2}$

Dunipace A, Brizendine E, Zhang W, Wilson M, Miller L, Katz B, Warrick J, Stookey G. 1995. Effect of aging on animal response to chronic fluoride exposure. J Dent Res. 74(1):358-368. http://dx.doi.org/10.1177/00220345950740011201

EFSA. 2012. EFSA Scientific Committee; Guidance on selected default values to be used by the EFSA Scientific Committee, Scientific Panels and Units in the absence of actual measured data. EFSA Journal. 10(3):2579. http://dx.doi.org/10.2903/j.efsa.2012.2579

Ekambaram P, Paul V. 2001. Calcium preventing locomotor behavioral and dental toxicities of fluoride by decreasing serum fluoride level in rats. Environ Toxicol Pharmacol. 9(4):141-146. http://dx.doi.org/10.1016/S1382-6689(00)00063-6 
Ekambaram P, Paul V. 2002. Modulation of fluoride toxicity in rats by calcium carbonate and by withdrawal of fluoride exposure. Pharmacol Toxicol. 90(2):53-58.

http://dx.doi.org/10.1034/j.1600-0773.2002.900201.x

Ekambaram P, Paul V. 2003. Effect of vitamin D on chronic behavioral and dental toxicities of sodium fluoride in rats. Fluoride. 36(3):189-197.

Ekstrand J, Boreus LO, de Chateau P. 1981. No evidence of transfer of fluoride from plasma to breast milk. Br Med J (Clin Res Ed). 283(6294):761-762.

http://dx.doi.org/10.1136/bmj.283.6294.761

Ekstrand J, Ehrnebo M. 1979. Influence of milk products on fluoride bioavailability in man. Eur J Clin Pharmacol. 16(3):211-215. http://dx.doi.org/10.1007/BF00562063

Ekstrand J, Ehrnebo M, Boreus LO. 1978. Fluoride bioavailability after intravenous and oral administration: importance of renal clearance and urine flow. Clin Pharmacol Ther. 23(3):329337. http://dx.doi.org/10.1002/cpt1978233329

Ekstrand J, Ziegler EE, Nelson SE, Fomon SJ. 1994. Absorption and retention of dietary and supplemental fluoride by infants. Adv Dent Res. 8(2):175-180.

http://dx.doi.org/10.1177/08959374940080020701

El-lethey H, Kamel M, Shaheed I. 2011. Perinatal exposure to sodium fluoride with emphasis on territorial aggression, sexual behaviour and fertility in male rats. Life Sci J. 8(2):686-694.

El-lethey HS, Kamel MM. 2011. Effects of black tea in mitigation of sodium fluoride potency to suppress motor activity and coordination in laboratory rats. J Am Sci. 7:243-254.

El-lethey HS, Kamel MM, Shaheed IB. 2010. Neurobehavioral toxicity produced by sodium fluoride in drinking water of laboratory rats. J Am Sci. 6(5):54-63.

El-lethey HS, Shaheed IB. 2011. Potential health impact of black tea against Na-F-Induced alterations in territorial aggression, sexual behaviour and fertility of male rats. Life Sci J. 8:828839.

Elliott L. 1967. Lack of effect of administration of fluoride on the central nervous system of rats. Acta Pharmacol Toxicol (Copenh). 25(3):323-328. http://dx.doi.org/10.1111/j.16000773.1967.tb01439.x

Flace P, Benagiano V, Vermesan D, Sabatini R, Inchingolo A, Auteri P, Ambrosi G, Tarullo A, Cagiano R. 2010. Effects of developmental fluoride exposure on rat ultrasonic vocalization, acoustic startle reflex and pre-pulse inhibition. Eur Rev Med Pharmacol Sci. 14(6):507-512.

Gao Q, Liu Y-J, Guan Z-Z. 2009a. Decreased learning and memory ability in rats with fluorosis: increased oxidative stress and reduced cholinesterase activity in the brain. Fluoride. 42(4):277.

Gao Q, Liu Y-J, Wu C-X, Long Y-G, Guan Z-Z. 2008a. Level of oxidative stress in rat brains and learning and memory function of rats with chronic fluorosis. Chinese Journal of Endemiology. 27(4):371-373. 
Gao Q, Liu Y, Wu C, Long Y, Guan Z. 2008b. Effects of fluoride on learning and memory and cholinesterase activity in rat brains. Chinese Journal of Endemiology. 27:128-130.

Gao Y, Liu L, Young L, Huan L, Jin H. 2009b. Effects of learning and memory of fluoride and the antagonism of selenium in rats. Studies of Trace Elements and Health. 26:1-3.

Gopal K, Saxena R, Gupta G, Rana M, Agrawal D. 2006. Fluoride induced alterations in neurobehavioural and cardiovascular responses in Rats. Journal of Advanced Zoology. 27(1):1.

Gui C-Z, Ran L-Y, Li J-P, Guan Z-Z. 2010. Changes of learning and memory ability and brain nicotinic receptors of rat offspring with coal burning fluorosis. Neurotoxicol Teratol. 32(5):536541. http://dx.doi.org/10.1016/j.ntt.2010.03.010

Guyatt G, Oxman AD, Akl EA, Kunz R, Vist G, Brozek J, Norris S, Falck-Ytter Y, Glasziou P, Jaeschke R. 2011a. GRADE guidelines: 1. Introduction-GRADE evidence profiles and summary of findings tables. J Clin Epidemiol. 64(4):383-394.

http://dx.doi.org/10.1016/j.jclinepi.2010.04.026

Guyatt GH, Oxman AD, Kunz R, Brozek J, Alonso-Coello P, Rind D, Devereaux P, Montori VM, Freyschuss B, Vist G. 2011b. GRADE guidelines 6. Rating the quality of evidenceimprecision. J Clin Epidemiol. 64(12):1283-1293. http://dx.doi.org/10.1016/i.jclinepi.2011.01.012

Guyatt GH, Oxman AD, Montori V, Vist G, Kunz R, Brozek J, Alonso-Coello P, Djulbegovic B, Atkins D, Falck-Ytter Y. 2011c. GRADE guidelines: 5. Rating the quality of evidencepublication bias. J Clin Epidemiol. 64(12):1277-1282.

http://dx.doi.org/10.1016/j.jclinepi.2011.01.011

Hamilton M. 1992. Water fluoridation: a risk assessment perspective. J Environ Health.27-32.

Han H, Du W, Zhou B, Zhang W, Xu G, Niu R, Sun Z. 2014. Effects of chronic fluoride exposure on object recognition memory and mRNA expression of SNARE complex in hippocampus of male mice. Biol Trace Elem Res. 158(1):58-64.

http://dx.doi.org/10.1007/s12011-014-9889-7

Haseman JK, Bailer AJ, Kodell RL, Morris R, Portier K. 2001. Statistical issues in the analysis of low-dose endocrine disruptor data. Toxicol Sci. 61(2):201-210.

http://dx.doi.org/10.1093/toxsci/61.2.201

Higgins J, Green S. 2011. Cochrane handbook for systematic reviews of interventions. Version 5.1. 0.http://handbook.cochrane.org/.

Hong J-H, Ge Y-M, Ning H-M. 2005. Effects of high fluoride and low iodine on learningmemory and TchE of brain in offspring rats. China Preventive Medicine. 6:489-491.

Hooijmans CR, Tillema A, Leenaars M, Ritskes-Hoitinga M. 2010. Enhancing search efficiency by means of a search filter for finding all studies on animal experimentation in PubMed. Lab Anim. 44(3):170-175. http://dx.doi.org/10.1258/la.2010.009117 
Iheozor-Ejiofor Z, Worthington HV, Walsh T, O’Malley L, Clarkson JE, Macey R, Alam R, Tugwell P, Welch V, Glenny AM. 2015. Water fluoridation for the prevention of dental caries. Cochrane Database Syst Rev. 6(CD010856).

International Programme on Chemical Safety (IPCS). 2002. Fluorides. Environmental Health Criteria 227. Geneva, Switzerland: World Health Organization http://www.inchem.org/documents/ehc/ehc/ehc227.htm.

Jain A, Mehta VK, Chittora R, Mahdi AA, Bhatnagar M. 2015. Melatonin ameliorates fluoride induced neurotoxicity in young rats: An In Vivo evidence. Asian Journal of Pharmaceutical and Clinical Research. 8(4):164-167.

Jetti R, Raghuveer C, Mallikarjuna RC. 2016. Protective effect of ascorbic acid and Ginkgo biloba against learning and memory deficits caused by fluoride. Toxicol Ind Health. 32(1):183187. http://dx.doi.org/10.1177/0748233713498460

Jiang C, Zhang S, Liu H, Guan Z, Zeng Q, Zhang C, Lei R, Xia T, Wang Z, Yang L et al. 2014a. Low glucose utilization and neurodegenerative changes caused by sodium fluoride exposure in rat's developmental brain. Neuromolecular Med. 16(1):94-105. http://dx.doi.org/10.1007/s12017$\underline{013-8260-\mathrm{Z}}$

Jiang S, Su J, Yao S, Zhang Y, Cao F, Wang F, Wang H, Li J, Xi S. 2014b. Fluoride and arsenic exposure impairs learning and memory and decreases mGluR5 expression in the hippocampus and cortex in rats. PLoS One. 9(4):e96041. http://dx.doi.org/10.1371/journal.pone.0096041

Kaminsky LS, Mahoney MC, Leach J, Melius J, Jo Miller M. 1990. Fluoride: benefits and risks of exposure. Crit Rev Oral Biol Medicine. 1(4):261-281. http://dx.doi.org/10.1177/10454411900010040501

Kivrak Y. 2012. Effects of fluoride on anxiety and depression in mice. Fluoride. 45(3):302-306.

Krauth D, Woodruff TJ, Bero L. 2013. Instruments for assessing risk of bias and other methodological criteria of published animal studies: a systematic review. Environ Health Perspect. 121(9):985-992. http://dx.doi.org/10.1289/ehp.1206389

Li M, Cui J, Gao Y, Zhang W, Sun L, Liu X, Liu Y, Sun D. 2015. Pathological changes and effect on the learning and memory ability in rats exposed to fluoride and aluminum. Toxicol Res. 4(5):1366-1373. http://dx.doi.org/10.1039/C5TX00050E

Liu F, Ma J, Zhang H, Liu P, Liu Y-P, Xing B, Dang Y-H. 2014. Fluoride exposure during development affects both cognition and emotion in mice. Physiol Behav. 124:1-7.

Liu W. 1989. Experimental study of behavior and cerebral morphology of rat pups generated by fluorotic female rat. Chinese Journal of Pathology. 18(4):290-292.

Liu Y-J, Gao G, Long Y-G, Yu Y-N, Guan Z. 2011. Influence of chronic fluorosis on expression of phospho-Elk-1 in rat brains. Chinese Journal of Endemiology. 30(3):251-255.

Liu YJ, Gao Q, Wu CX, Guan ZZ. 2010. Alterations of nAChRs and ERK1/2 in the brains of rats with chronic fluorosis and their connections with the decreased capacity of learning and memory. Toxicol Lett. 192(3):324-329. http://dx.doi.org/10.1016/j.toxlet.2009.11.002 
Liu YJ, Gao Q, Wu CX, Long YG, Guan ZZ. 2009. Modified expression of extracellular signalregulated protein kinase signal transduction in rat brains and changed capacity of learning and memory of rats with chronic fluorosis. Chinese Journal of Endemiology. 28(1):32-35.

Ma J, Liu F, Liu P, Dong YY, Chu Z, Hou TZ, Dang YH. 2015. Impact of early developmental fluoride exposure on the peripheral pain sensitivity in mice. Int J Dev Neurosci. 47(Pt B):165171. http://dx.doi.org/10.1016/j.ijdevneu.2015.09.005

Mullenix PJ, Denbesten PK, Schunior A, Kernan WJ. 1995. Neurotoxicity of sodium fluoride in rats. Neurotoxicol Teratol. 17(2):169-177. http://dx.doi.org/10.1016/0892-0362(94)00070-T

National Research Council (NRC). 1993. Health effects of ingested fluoride. Washington, DC: National Academies Press.

National Research Council (NRC). 2006. Committee on Fluoride in Drinking Water, Board on Environmental Studies and Toxicology. Fluoride in drinking water: a scientific review of EPA's standards. Washington, DC: National Academies Press.

National Toxicology Program (NTP). 2015a. Handbook for conducting a literature-based health assessment using Office of Health Assessment and Translation (OHAT) approach for systematic review and evidence integration. National Institute of Environmental Health Sciences, Division of the National Toxicology Program, Office of Health Assessment and Translation (OHAT).https://ntp.niehs.nih.gov/pubhealth/hat/review/index-2.html.

National Toxicology Program (NTP). 2015b. OHAT risk of bias tool.https://ntp.niehs.nih.gov/pubhealth/hat/review/index-2.html.

Niu R, Liu S, Wang J, Zhang J, Sun Z, Wang J. 2014. Proteomic analysis of hippocampus in offspring male mice exposed to fluoride and lead. Biol Trace Elem Res. 162(1-3):227-233. http://dx.doi.org/10.1007/s12011-014-0117-2

Niu R, Sun Z, Cheng Z, Li Z, Wang J. 2009. Decreased learning ability and low hippocampus glutamate in offspring rats exposed to fluoride and lead. Environ Toxicol Pharmacol. 28(2):254258. http://dx.doi.org/10.1016/j.etap.2009.04.012

Niu R, Sun Z, Wang J, Cheng Z, Wang J. 2008. Effects of fluoride and lead on locomotor behavior and expression of nissl body in brain of adult rats. Fluoride. 41(4):276-282.

Ophaug RH, Singer L, Harland BF. 1980. Estimated fluoride intake of average two-year-old children in four dietary regions of the United States. J Dent Res. 59(5):777-781.

http://dx.doi.org/10.1177/00220345800590050501

Paul V, Ekambaram P, Jayakumar A. 1998. Effects of sodium fluoride on locomotor behavior and a few biochemical parameters in rats. Environ Toxicol Pharmacol. 6(3):187-191.

http://dx.doi.org/10.1016/S1382-6689(98)00033-7

Pereira M, Dombrowski PA, Losso EM, Chioca LR, Da Cunha C, Andreatini R. 2011. Memory impairment induced by sodium fluoride is associated with changes in brain monoamine levels. Neurotox Res. 19(1):55-62. http://dx.doi.org/10.1007/s12640-009-9139-5 
Raghu J, Raghuveer VC, Rao MC, Somayaji NS, Babu PB. 2013. The ameliorative effect of ascorbic acid and Ginkgo biloba on learning and memory deficits associated with fluoride exposure. Interdiscip Toxicol. 6(4):217-221. http://dx.doi.org/10.2478/intox-2013-0032

Reddy MM, Karnati PR. 2015. Protective effects of aqueous extract of fruit pulp of Tamarindus indica on motor activity and metabolism of the gastrocnemius muscle of rats treated with fluoride. International Journal of Toxicological and Pharmacological Research. 7(5):241-246.

Rumiantsev G, Novikov S, Mel'nikova N, Levchenko N, Kozeeva E. 1988. Experimental study of the biological effect of salts of hydrofluosilicic acid. Gig Sanit. (11):80-82.

Sarkozi K, Horvath E, Vezer T, Papp A, Paulik E. 2015. Behavioral and general effects of subacute oral arsenic exposure in rats with and without fluoride. Int J Environ Health Res. 25(4):418-431. http://dx.doi.org/10.1080/09603123.2014.958138

Scientific Committee on Health and Environmental Risks (SCHER). 2011. European Commission Directorate-General for Health and Consumers, Scientific Committees. Critical review of any new evidence on the hazard profile, health effects, and human exposure to fluoride and the fluoridating agents of drinking water. http://ec.europa.eu/health/scientific_committees/environmental_risks/docs/scher_o_139.pdf.http: //ec.europa.eu/health/scientific_committees/environmental_risks/docs/scher_o_139.pdf.

Shen X, Zhang Z, Xu X. 2004. Effect of iodine and selenium on learning memory impairment induced by fluorosis and blood biochemical criterion of rats. Occupation and Health. 1:6-8.

Shen YW, Taves DR. 1974. Fluoride concentrations in the human placenta and maternal and cord blood. Am J Obstet Gynecol. 119(2):205-207. http://dx.doi.org/10.1016/00029378(74)90035-0

Shulman ER, Vallejo M. 1990. Effect of gastric contents on the bioavailability of fluoride in humans. Pediatr Dent. 12(4):237-240.

Spak C, Ekstrand J, Zylberstein D. 1982. Bioavailability of fluoride added to baby formula and milk. Caries Res. 16(3):249-256. http://dx.doi.org/10.1159/000260605

Sun Z, Liu F, Wu Ln, Lu Y, Yu D. 2008. Effects of high fluoride drinking water on the cerebral function of mice. Fluoride. 41(2):148-151.

Sutton M, Kiersey R, Farragher L, Long J. 2015. Health effects of water fluoridation: An evidence review. Ireland: Republic of Ireland Department of Health.http://www.hrb.ie/uploads/tx_hrbpublications/Health_Effects_of_Water_Fluoridation.pdf

Trautner K, Einwag J. 1989. Influence of Milk and Food on Fluoride Bioavailability from NaF and Na2FPO 3 in Man. J Dent Res. 68(1):72-77. http://dx.doi.org/10.1177/00220345890680011201

Turner C, Hasegawa K, Zhang W, Wilson M, Li Y, Dunipace A. 1995. Fluoride reduces bone strength in older rats. J Dent Res. 74(8):1475-1481. http://dx.doi.org/10.1177/00220345950740080701 
U.S. Department of Health and Human Services (US DHHS). 2015. U.S. Public Health Service recommendation for fluoride concentration in drinking water for the prevention of dental caries. Public Health Rep. 130:318-331. http://dx.doi.org/10.1177/003335491513000408

U.S. Environmental Protection Agency (US EPA). 1988. Recommendations for and Documentation of Biological Values for Use in Risk Assessment Washington D.C.: U.S. Environmental Protection Agency, Office of Research and Development, Office of Health and Environmental Assessment, Environmental Criteria and Assessment Office EPA/600/6-87/008 (NTIS PB88179874).http://cfpub.epa.gov/ncea/risk/recordisplay.cfm?deid=34855.

U.S. Environmental Protection Agency (US EPA). 1994. Methods for derivation of inhalation reference concentrations (RfCs) and application of inhalation dosimetry. Washington, DC: U.S. Environmental Protection Agency, Office of Research and Development, Office of Health and Environmental Assessment, Environmental Criteria and Assessment Office. EPA/600/890/066F.https://cfpub.epa.gov/ncea/risk/recordisplay.cfm?deid=71993.

U.S. Environmental Protection Agency (US EPA). 2010a. Fluoride: Dose-response analysis for non-cancer effects. Washington: Office of Water, Health and Ecological Criteria Division. 820R-10-018.https:/www.epa.gov/sites/production/files/2015-09/documents/fluoride-studysummaries.pdf.

U.S. Environmental Protection Agency (US EPA). 2010b. Fluoride: Exposure and relative source contribution analysis. Washington D.C.: Office of Water, Health, and Ecological Criteria Division. 820-R-10-015.http://www.epa.gov/dwstandardsregulations/fluoride-risk-assessmentand-relative-source-contribution.

U.S. Environmental Protection Agency (US EPA). 2013. Basic information about fluoride in drinking water: Review of fluoride drinking water standard.https://www.epa.gov/dwsixyearreview/review-fluoride-drinking-water-regulation.

Vesterinen H, Sena E, Egan K, Hirst T, Churolov L, Currie G, Antonic A, Howells D, Macleod M. 2014. Meta-analysis of data from animal studies: a practical guide. J Neurosci Methods. 221:92-102. http://dx.doi.org/10.1016/j.jneumeth.2013.09.010

Vishnevskii VL, EL Nichnykh LN. 1969. A toxicological and morphological characterization of the action of different concentrations of inhaled hydrogen fluoride on the body. Tr Tsentr Nauchno-Issled Proektn-Konstr In. 2:143-147.

Vorhees CV, Williams MT. 2006. Morris water maze: procedures for assessing spatial and related forms of learning and memory. Nat Protoc. 1(2):848-858.

http://dx.doi.org/10.1038/nprot.2006.116

Vorhees CV, Williams MT. 2014. Assessing spatial learning and memory in rodents. ILAR J. 55(2):310-332. http://dx.doi.org/10.1093/ilar/ilu013

Wang G, Li J, Zhu H, Zhu J. 2006. Effect of different doses of chronic exposure of fluoride on rat learning and memory behavior. Studies of Trace Elements and Health. 23(2):1-2.

Wang J, Ge Y, Ning H, Wang S. 2004. Effects of high fluoride and low iodine on biochemical indexes of the brain and learning-memory of offspring rats. Fluoride. 37(3):201-208. 
Waterhouse C, Taves D, Munzer A. 1980. Serum inorganic fluoride: changes related to previous fluoride intake, renal function and bone resorption. Clin Sci. 58(2):145-152.

http://dx.doi.org/10.1042/cs0580145

Wei N, Dong Y, Wang Y, Guan Z. 2014. Effects of chronic fluorosis on neurobehavioral development in offspring of rats and antagonistic effect of vitamin E. Chinese Journal of Endemiology. 33(2):125-128.

Whitford GM. 1996. The Metabolism and Toxicity of Fluoride. Monographs in Oral Science,. 2nd ed. New York: Karger Publishers. p. I-XIII.

Whitford GM, Pashley DH. 1984. Fluoride absorption: the influence of gastric acidity. Calcif Tissue Int. 36(3):302-307. http://dx.doi.org/10.1007/BF02405334

Whitford GM, Whitford JL, Hobbs SH. 2009. Appetitive-based learning in rats: lack of effect of chronic exposure to fluoride. Neurotoxicol Teratol. 31(4):210-215.

http://dx.doi.org/10.1016/j.ntt.2009.02.003

World Health Organization (WHO). 1994. Fluorides and oral health. Report of a WHO Expert Committee on Oral Health Status and Fluoride Use. Geneva, Switzerland: WHO (World Health Organization). 846.http://apps.who.int/iris/bitstream/10665/39746/1/WHO_TRS_846.pdf

Wu C, Gu X, Ge Y, Zhang J, Wang J. 2006. Effects of high fluoride and arsenic on brain biochemical indexes and learning-memory in rats. Fluoride. 39(4):274-279.

Wu N, Zhao Z, Gao W, Li X. 2008. Behavioral teratology in rats exposed to fluoride. Fluoride. 41(2):129-133.

$\mathrm{Xu}$ X, Zhang Z, Shen X. 2001. The influence of fluorosis on learning-memory behaviors of mice and the activities of SOD and the content of MDA in the brain. China Public Health. 17(1):8-10.

Zhang C, Ren C, Chen H, Geng R, Fan H, Zhao H, Guo K, Geng D. 2013. The analog of Ginkgo biloba extract 761 is a protective factor of cognitive impairment induced by chronic fluorosis. Biol Trace Elem Res. 153(1-3):229-236. http://dx.doi.org/10.1007/s12011-013-9645-4

Zhang J, Zhu W, Zhang Z. 2009. The effect of fluorine exposure of pregnant rats on the learning and memory capabilities of baby rats (Influence of fluorosis on learning memory of pup rats and activities of SOD and content of MDA in brain). Chin J Public Health. 25:1347-1348.

Zhang Z, Shen X, Xu X. 2001. Effects of selenium on the damage of learning-memory ability of mice induced by fluoride. Wei sheng yan jiu. 30(3):144-146.

Zhang Z, Sun Y, Zheng X. 2015. The synaptic mechanism of learning-memory injury induced by chronic fluorosis in brain. Journal of Zhejiang Normal University: Natural Sciences. 38(1):1-8.

Zhang Z, Xu X, Shen X. 1999. Effect of fluoride exposure on synaptic structure of brain areas related to learning-memory in mice. Wei sheng yan jiu. 28(4):210-212.

Zhu Y-1, Zheng Y-j, LV XM, Ma Y, Zhang J. 2012. Effects of fluoride exposure on performance in water labyrinth and monoamine neurotransmitters of rats. Journal of Xinjiang Medical University. 3:014. 


\section{Appendix A. Electronic Database Search Strategies}

\section{Table of Contents}

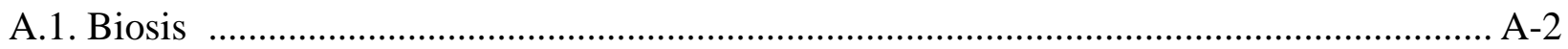

A.2. EMBASE …............................................................................................... A-4

A.3. PsycINFO ......................................................................................................... A-5

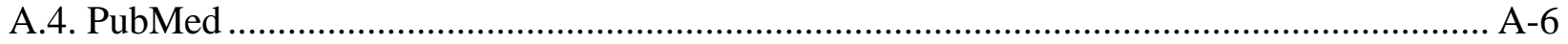

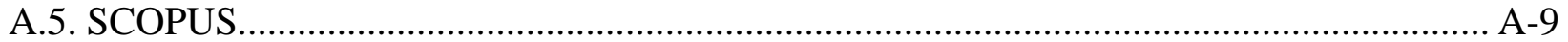

A.6. Web of Science ........................................................................................................... A-10

\section{Tables}

Table A-1. Search Strategy for BIOSIS .......................................................................... A-2

Table A-2. Search Strategy for EMBASE ........................................................................ A-4

Table A-3. Search Strategy for PsycINFO ....................................................................... A-5

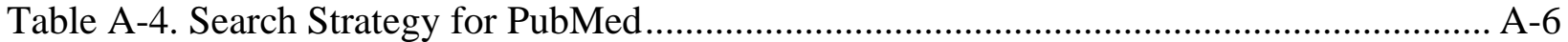

Table A-5. Search Strategy for SCOPUS ..................................................................... A-9

Table A-6. Search Strategy for Web of Science .......................................................... A-10 
Systematic Literature Review on the Effects of Fluoride on Learning and Memory in Animal Studies

\section{A.1. Biosis}

Table A-1. Search Strategy for BIOSIS

\begin{tabular}{|c|c|c|}
\hline Search ID & Search Terms & $\begin{array}{c}\text { Date: Number of } \\
\text { Records }\end{array}$ \\
\hline \# 4 & \#1 AND \#2 AND \#3 & $\begin{array}{l}\text { 2/18/2015: } 899 \\
\text { 1/14/2016 } \\
\text { update: } 21\end{array}$ \\
\hline $\begin{array}{l}\# 1 \\
\text { Animal } \\
\text { Studies }\end{array}$ & 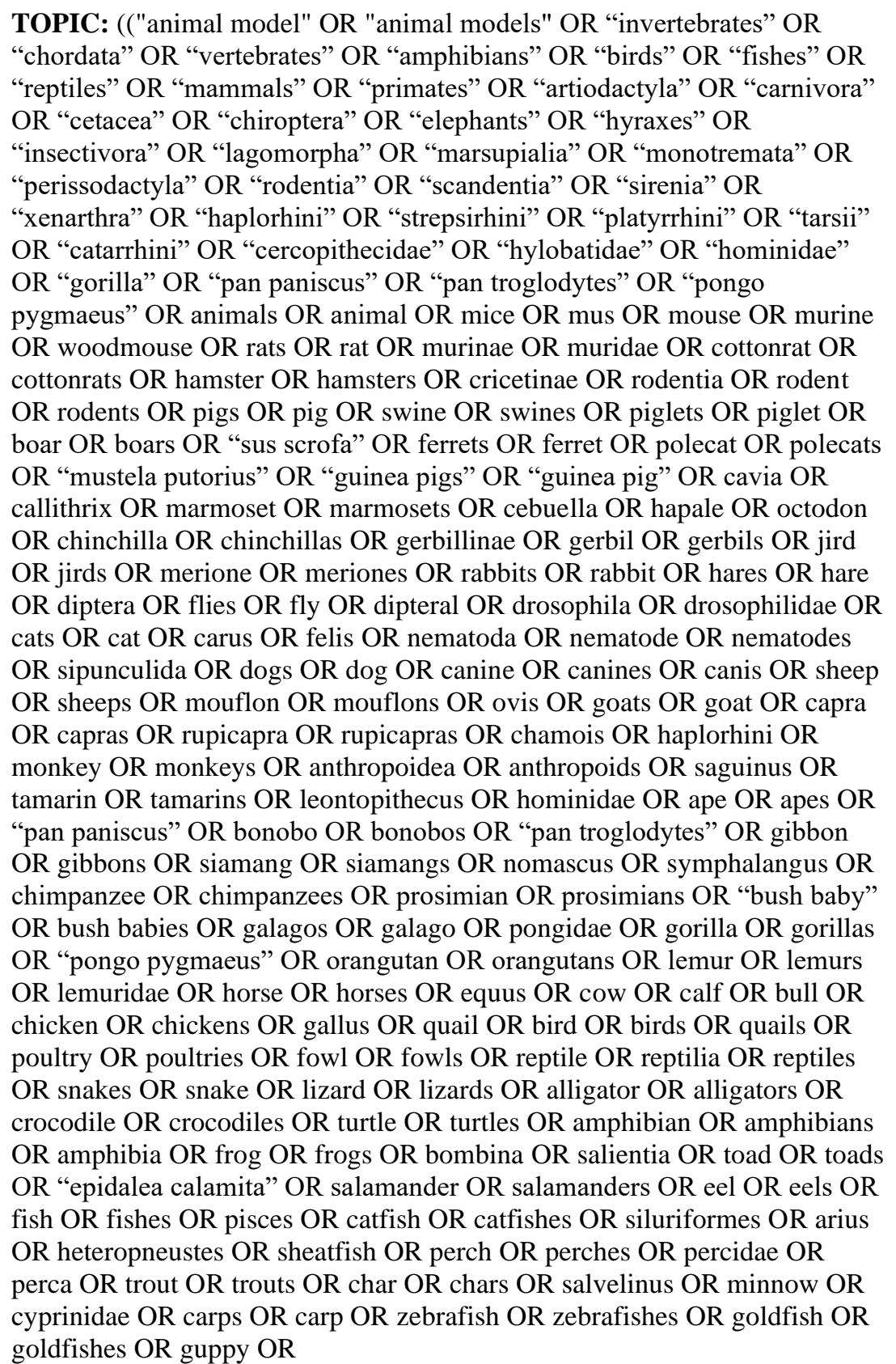 & $\begin{array}{l}\text { 2/18/2015: } \\
218,068,453 \\
1 / 14 / 2016 \text { update: } \\
150,971\end{array}$ \\
\hline
\end{tabular}


Systematic Literature Review on the Effects of Fluoride on Learning and Memory in Animal Studies

\begin{tabular}{|c|c|c|}
\hline Search ID & Search Terms & $\begin{array}{c}\text { Date: Number of } \\
\text { Records }\end{array}$ \\
\hline $\begin{array}{l}\# 2 \\
\text { Neurological } \\
\text { Outcomes }\end{array}$ & 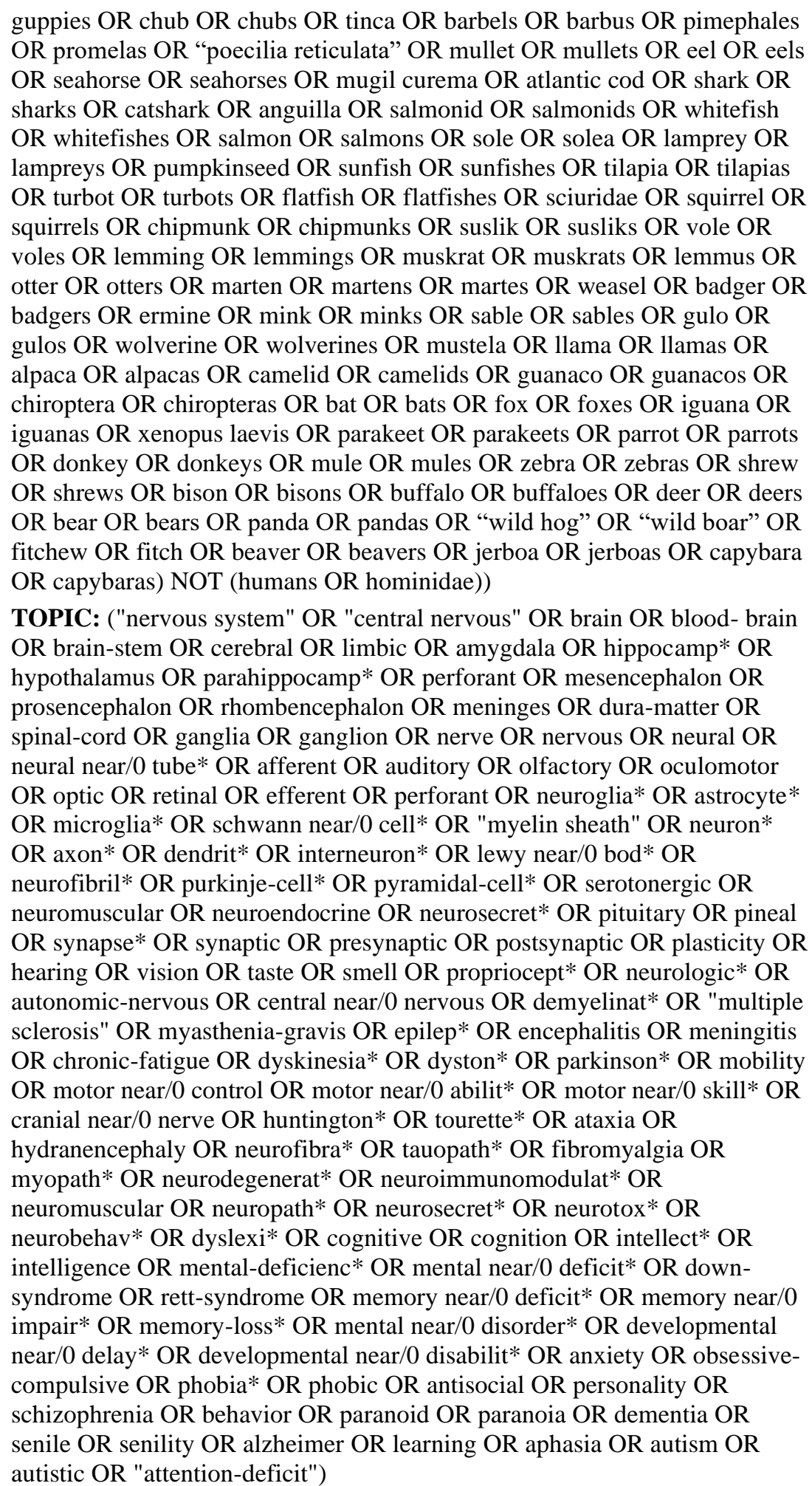 & $\begin{array}{l}\text { 2/18/2015: } \\
5,947,883 \\
\text { 1/14/2016 update: } \\
168,166 \\
\text { 2/18/2015: } 21,679\end{array}$ \\
\hline
\end{tabular}


Systematic Literature Review on the Effects of Fluoride on Learning and Memory in Animal Studies

\begin{tabular}{lll}
\hline \multicolumn{1}{c}{ Search ID } & \multicolumn{1}{c}{ Search Terms } & $\begin{array}{c}\text { Date: Number of } \\
\text { Records }\end{array}$ \\
\hline \# 3 & TITLE=(fluorid* OR flurid* OR fluorin* OR florin* OR fluorosis) NOT & $1 / 14 / 2016$ update: \\
Fluoride & TOPIC=((18F OR F-18 OR "fluorine-18" OR 19F OR F-19 OR "fluorine-19" & 450 \\
& OR (PET AND scan) OR f-labeled OR imaging OR radioligand*)) & \\
\hline
\end{tabular}

\section{A.2. EMBASE}

Search Note: Animal studies were restricted to the traditional animal models. Any other animal studies would likely be picked up in the other databases.

Table A-2. Search Strategy for EMBASE

\begin{tabular}{|c|c|c|}
\hline Search ID & Search Terms & $\begin{array}{l}\text { Date: Number of } \\
\text { Records }\end{array}$ \\
\hline \# 4 & \#1 AND \#2 AND \#3 & $\begin{array}{l}\text { 1/7/ 2015: } 306 \\
\text { 1/14/2016 } \\
\text { update: } 25\end{array}$ \\
\hline $\begin{array}{l}\text { \# } 1 \\
\text { Animal } \\
\text { Studies }\end{array}$ & $\begin{array}{l}\text { 'animal model'/exp OR rats OR rat OR mice OR mouse OR rodent* OR } \\
\text { murin* }\end{array}$ & $\begin{array}{l}\text { 1/7/2015: } 3,389,269 \\
\text { 1/14/2016 update: } \\
137,941\end{array}$ \\
\hline $\begin{array}{l}\# 2 \\
\text { Neurological } \\
\text { Outcomes }\end{array}$ & $\begin{array}{l}\text { 'nervous system'/exp OR 'nervous system' OR 'central-nervous' OR } \\
\text { 'brain'/exp OR brain OR 'blood brain' OR 'brain stem'/exp OR 'brain stem' } \\
\text { OR cerebral OR cortical OR limbic OR 'amygdala'/exp OR amygdala OR } \\
\text { hippocamp* OR 'hypothalamus'/exp OR hypothalamus OR parahippocamp* } \\
\text { OR 'mesencephalon'/exp OR mesencephalon OR 'prosencephalon'/exp OR } \\
\text { prosencephalon OR 'rhombencephalon'/exp OR rhombencephalon OR } \\
\text { 'meninges'/exp OR meninges OR 'dura matter' OR 'spinal cord'/exp OR } \\
\text { 'spinal cord' OR 'ganglia'/exp OR ganglia OR 'ganglion'/exp OR ganglion } \\
\text { OR 'nerve'/exp OR nerve OR neural OR 'neural tube'/exp OR 'neural tube' } \\
\text { OR afferent OR auditory OR olfactory OR oculomotor OR optic OR } \\
\text { 'retinal'/exp OR retinal OR efferent OR perforant OR neuroglia* OR } \\
\text { astrocyte* OR microglia* OR schwann NEAR/0 cell* OR 'myelin } \\
\text { sheath'/exp OR 'myelin sheath' OR neuron* OR axon* OR dendrit* OR } \\
\text { interneuron* OR lewy NEXT/0 bod* OR neurofibril* OR purkinje NEXT/0 } \\
\text { cell* OR pyramidal NEXT/0 cell* OR serotonergic OR neuromuscular OR } \\
\text { neuroendocrine OR neurosecret* OR 'pituitary'/exp OR pituitary OR pineal } \\
\text { OR synapse* OR synaptic OR presynaptic OR postsynaptic OR } \\
\text { 'plasticity'/exp OR plasticity OR 'hearing'/exp OR hearing OR 'vision'/exp } \\
\text { OR vision OR 'taste'/exp OR taste OR 'smell'/exp OR smell OR } \\
\text { propriocept* OR nervous AND system AND ('diseases'/exp OR diseases) } \\
\text { OR neurologic* OR 'autonomic nervous' OR 'central nervous' OR } \\
\text { demyelinat* OR 'multiple sclerosis'/exp OR 'multiple sclerosis' OR } \\
\text { 'myasthenia gravis'/exp OR 'myasthenia gravis' OR epilep* OR } \\
\text { 'encephalitis'/exp OR encephalitis OR 'meningitis'/exp OR meningitis OR } \\
\text { 'chronic fatigue' OR dyskinesia* OR dyston* OR parkinson* OR mobility } \\
\text { OR 'motor control'/exp OR 'motor control' OR motor NEXT/0 abilit* OR } \\
\text { motor NEXT/0 skill* OR 'cranial nerve'/exp OR 'cranial nerve' OR } \\
\text { huntington* OR tourette* OR 'ataxia'/exp OR ataxia OR } \\
\text { 'hydranencephaly'/exp OR hydranencephaly OR neurofibra* OR tauopath* }\end{array}$ & $\begin{array}{l}\text { 1/7/2015: 4,078,217 } \\
\text { 1/14/2016 update: } \\
237,733\end{array}$ \\
\hline
\end{tabular}


Systematic Literature Review on the Effects of Fluoride on Learning and Memory in Animal Studies

\begin{tabular}{|c|c|c|}
\hline Search ID & Search Terms & $\begin{array}{l}\text { Date: Number of } \\
\text { Records }\end{array}$ \\
\hline & $\begin{array}{l}\text { OR 'fibromyalgia'/exp OR fibromyalgia OR myopath* OR neurodegenerat* } \\
\text { OR neuroimmunomodulat* OR neuromuscular OR neuropath* OR } \\
\text { neurosecret* OR neurotox* OR neurobehav* OR dyslexi* OR cognitive OR } \\
\text { 'cognition'/exp OR cognition OR intellect* OR 'intelligence'/exp OR } \\
\text { intelligence OR mental NEAR/0 deficienc* OR mental NEAR/0 deficit* } \\
\text { OR 'down syndrome'/exp OR 'down syndrome' OR 'rett syndrome'/exp OR } \\
\text { 'rett syndrome' OR memory NEAR/0 deficit* OR memory NEAR/0 impair* } \\
\text { OR memory NEAR/0 loss* OR mental AND disorders OR developmental } \\
\text { AND disabilities OR developmental NEAR/0 delay* OR developmental } \\
\text { NEAR/0 disabilit* OR 'anxiety'/exp OR anxiety OR 'obsessive compulsive' } \\
\text { OR phobia* OR phobic OR antisocial OR 'personality'/exp OR personality } \\
\text { OR 'schizophrenia'/exp OR schizophrenia OR 'behavior'/exp OR behavior } \\
\text { OR 'paranoid'/exp OR paranoid OR 'paranoia'/exp OR paranoia OR } \\
\text { 'dementia'/exp OR dementia OR senile OR 'senility'/exp OR senility OR } \\
\text { alzheimer OR 'learning'/exp OR learning OR 'aphasia'/exp OR aphasia OR } \\
\text { 'autism'/exp OR autism OR autistic OR 'attention deficit'/exp OR 'attention } \\
\text { deficit' }\end{array}$ & \\
\hline $\begin{array}{l}\# 3 \\
\text { Fluoride }\end{array}$ & $\begin{array}{l}\text { 2/18/2015: fluoride:de OR fluorid* NOT ('f labeled' OR 'fluorine 18'/exp } \\
\text { OR 'fluorine 18' OR 'imaging'/exp OR imaging OR radioligand*) 1/14/2016 } \\
\text { update: fluoride:de OR fluorid* OR flurid* OR fluorinated OR } \\
\text { 'fluorosis'/exp OR fluorosis NOT ('18f'/exp OR 18f OR 'f 18'/exp OR 'f 18' } \\
\text { OR 19f OR 'f 19' OR 'fluorine-19'/exp OR 'fluorine-19' OR (pet AND scan) } \\
\text { OR 'f labeled' OR 'fluorine 18'/exp OR 'fluorine 18' OR 'imaging' OR } \\
\text { 'imaging'/exp OR imaging OR radioligand*) AND [2015-2016]/py }\end{array}$ & $\begin{array}{l}\text { 1/7/2015: } 59,675 \\
1 / 14 / 2016 \text { update: } \\
1,635\end{array}$ \\
\hline
\end{tabular}

\section{A.3. PsycINFO}

Search Note: Given how studies fluoride studies were included in this database, the search was not restricted to neurological outcomes in animals.

Table A-3. Search Strategy for PsycINFO

\begin{tabular}{|c|c|c|}
\hline $\begin{array}{l}\text { Search } \\
\text { ID }\end{array}$ & Search Terms & $\begin{array}{c}\text { Date: Number of } \\
\text { Records }\end{array}$ \\
\hline \# 1 & $\begin{array}{l}\text { 1/7/2015: Title: fluorid* OR flurid* OR fluorin* OR florin* } \\
\text { 1/14/2016 update: Title: fluorid* OR flurid* OR fluorin* OR florin* OR } \\
\text { fluorosis OR Abstract: fluorid* OR flurid* OR fluorin* OR florin* OR } \\
\text { fluorosis AND Year: } 2015 \text { To } 2016\end{array}$ & $\begin{array}{l}\text { 1/7/2015: } 74 \\
\text { 1/14/2016 update: } 36\end{array}$ \\
\hline
\end{tabular}


Systematic Literature Review on the Effects of Fluoride on Learning and Memory in Animal Studies

\section{A.4. PubMed}

Table A-4. Search Strategy for PubMed

\begin{tabular}{|c|c|c|}
\hline Search ID & Search Terms & $\begin{array}{l}\text { Date: Number of } \\
\text { Records }\end{array}$ \\
\hline \# 4 & \#1 AND \#2 AND \#3 & $\begin{array}{l}\text { 1/7/2015: } 2417 \\
\text { 1/14/2016 update: } \\
53\end{array}$ \\
\hline $\begin{array}{l}\# 1 \\
\text { Animal } \\
\text { Studies } \\
\text { [from } \\
\text { Hooijmans } \\
\text { et al. } \\
(2010) \text { ] }\end{array}$ & 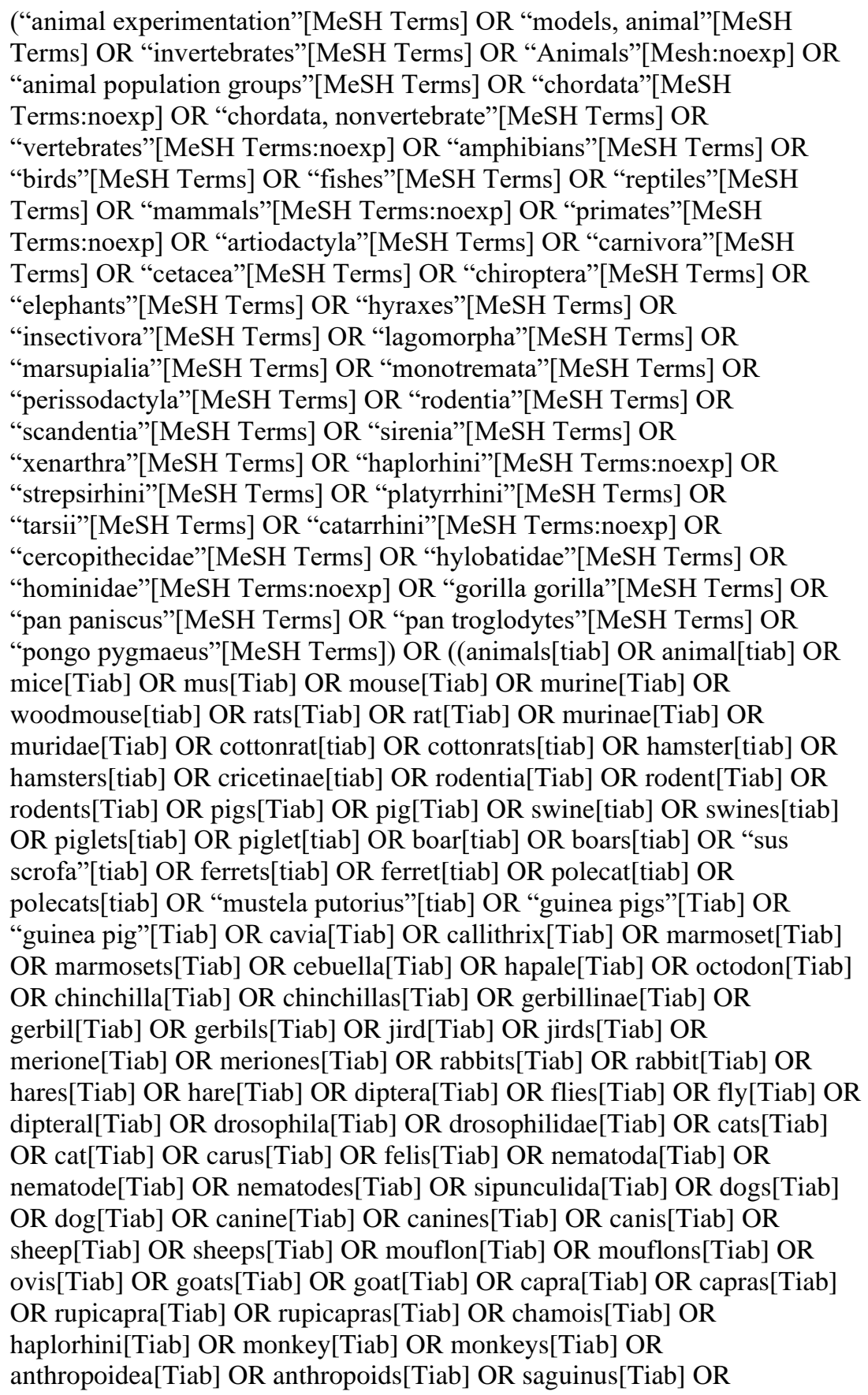 & $\begin{array}{l}\text { 1/7/2015: } 5,723,242 \\
\text { 1/14/2016 update: } \\
209,076\end{array}$ \\
\hline
\end{tabular}


Systematic Literature Review on the Effects of Fluoride on Learning and Memory in Animal Studies

\begin{tabular}{|c|c|c|}
\hline Search ID & Search Terms & $\begin{array}{l}\text { Date: Number of } \\
\text { Records }\end{array}$ \\
\hline & 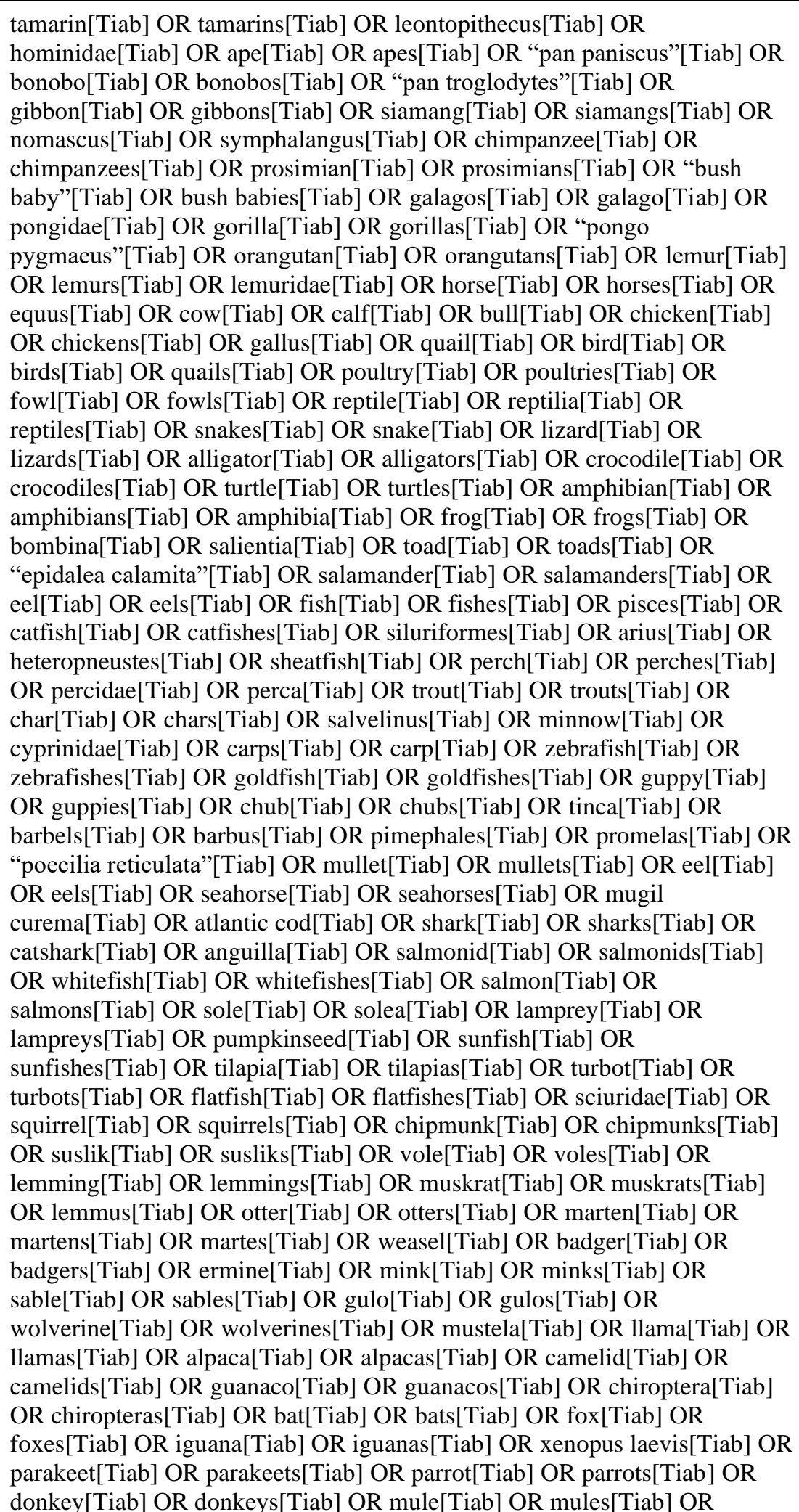 & \\
\hline
\end{tabular}


Systematic Literature Review on the Effects of Fluoride on Learning and Memory in Animal Studies

\begin{tabular}{|c|c|c|}
\hline Search ID & Search Terms & $\begin{array}{l}\text { Date: Number of } \\
\text { Records }\end{array}$ \\
\hline & $\begin{array}{l}\text { zebra[Tiab] OR zebras[Tiab] OR shrew[Tiab] OR shrews[Tiab] OR } \\
\text { bison[Tiab] OR bisons[Tiab] OR buffalo[Tiab] OR buffaloes[Tiab] OR } \\
\text { deer[Tiab] OR deers[Tiab] OR bear[Tiab] OR bears[Tiab] OR panda[Tiab] } \\
\text { OR pandas[Tiab] OR “wild hog”[Tiab] OR “wild boar”[Tiab] OR } \\
\text { fitchew[Tiab] OR fitch[Tiab] OR beaver[Tiab] OR beavers[Tiab] OR } \\
\text { jerboa[Tiab] OR jerboas[Tiab] OR capybara[Tiab] OR capybaras[Tiab]) } \\
\text { NOT medline[sb]) }\end{array}$ & \\
\hline $\begin{array}{l}\# 2 \\
\text { Neurologica } \\
1 \text { Outcomes }\end{array}$ & 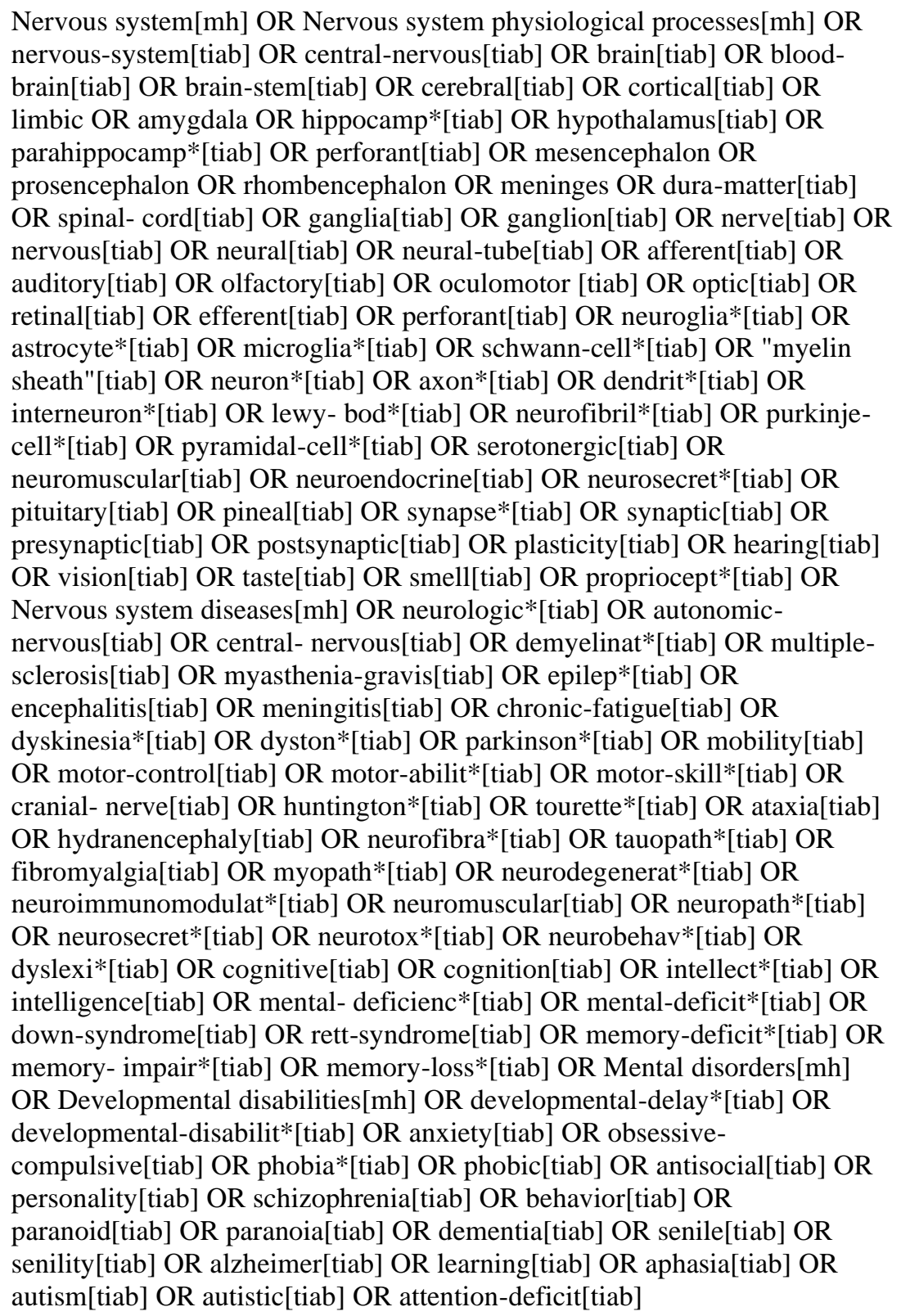 & $\begin{array}{l}\text { 1/7/2015: } 5,326,936 \\
\text { 1/14/2016 update: } \\
276,401\end{array}$ \\
\hline $\begin{array}{l}\text { \# } 3 \\
\text { Fluoride }\end{array}$ & $\begin{array}{l}\text { 1/7/2015: (Fluorides[mh:noexp] OR fluorides, topical[mh] OR sodium } \\
\text { fluoride[mh] OR fluorid*[tiab] OR flurid*[tiab] OR fluorin*[tiab] OR } \\
\text { florin*[tiab]) NOT (f-labeled[tiab] OR "fluorine- } 18 \text { "[tiab] OR imaging[tiab] } \\
\text { OR radioligand*[tiab]) 1/14/2016 update: (Fluorides[mh:noexp] OR }\end{array}$ & $\begin{array}{l}\text { 1/7/2015: } 58,732 \\
1 / 14 / 2016 \text { update: } \\
2813\end{array}$ \\
\hline
\end{tabular}


Systematic Literature Review on the Effects of Fluoride on Learning and Memory in Animal Studies

\begin{tabular}{ccc}
\hline Search ID & Search Terms & $\begin{array}{c}\text { Date: Number of } \\
\text { Records }\end{array}$ \\
\hline
\end{tabular}

fluorides, topical[mh] OR sodium fluoride[mh] OR fluorid*[tiab] OR

flurid*[tiab] OR fluorin*[tiab] OR florin*[tiab]) NOT (18F[tiab] OR f-

18[tiab] OR 19F[tiab] OR f-19[tiab] OR f-labeled[tiab] OR "fluorine-

18"[tiab] OR "fluorine-19"[tiab] OR pet-scan[tiab] OR imaging[tiab] OR

radioligand*[tiab]) Sort by: PublicationDate Filters: Publication date from

2015/01/01 to $2016 / 12 / 31$

\section{A.5. SCOPUS}

Table A-5. Search Strategy for SCOPUS

\begin{tabular}{|c|c|c|}
\hline Search ID & Search Terms & $\begin{array}{l}\text { Date: Number of } \\
\text { Records }\end{array}$ \\
\hline \# 4 & \#1 AND \#2 AND \#3 & $\begin{array}{l}\text { 1/7/2015: } 1759 \\
\text { 1/14/2016 update: } 89\end{array}$ \\
\hline $\begin{array}{l}\text { \# } 1 \\
\text { Animal } \\
\text { Studies }\end{array}$ & $\begin{array}{l}\text { TITLE-ABS (animal* OR mice OR mus OR mouse OR murine OR rats } \\
\text { OR rat OR murinae OR muridae OR "cotton rat" OR "cotton rats" OR } \\
\text { hamster* OR criceticae OR rodent* OR pigs OR pig OR swine* OR } \\
\text { piglet* OR "guinea pigs" OR "guinea pig" OR cavia OR callithrix OR } \\
\text { marmoset* OR cebuella OR hapale OR octodon OR chinchilla* OR } \\
\text { gerbillinae OR gerbil* OR rabbit* OR hares OR hare OR cats OR cat OR } \\
\text { carus OR felis OR dogs OR dog OR canine* OR canis OR sheep* OR } \\
\text { mouflon* OR ovis OR goat* OR capra OR haplorhini OR monkey* OR } \\
\text { anthropoid* OR saguinus OR tamarin* OR leontopithecus OR hominidae } \\
\text { OR ape OR apes OR "pan paniscus" OR bonobo* OR "pan troglodytes" } \\
\text { OR gibbon* OR nomascus OR symphalangus OR chimpanzee* OR } \\
\text { prosimian* OR pongidae OR gorilla* OR "pongo pygmaeus" OR } \\
\text { orangutan* OR lemur* OR lemuridae OR horse* OR equus OR cow OR } \\
\text { calf OR bull OR chicken* OR gallus OR quail* OR bird OR birds OR } \\
\text { poultry OR fowl OR fowls OR reptil* OR snake* OR lizard* OR turtle* } \\
\text { OR amphibia* OR frog* OR xenopus OR bombina OR salientia OR } \\
\text { toad* OR "epidalea calamita" OR salamander* OR fish OR fishes OR } \\
\text { pisces OR catfish OR perch OR percidae OR perca OR trout OR char OR } \\
\text { salmon OR salvelinus OR minnow* OR cyprinidae OR carp OR } \\
\text { zebrafish OR "zebra fish" OR nematode* OR elegans OR diptera OR } \\
\text { flies OR dipteral OR drosophil* OR squirrel* OR chipmunk* OR bear } \\
\text { OR bears OR ursidae OR dolphin* OR porpoise* OR whale* OR } \\
\text { cetacea) }\end{array}$ & $\begin{array}{l}\text { 1/7/2015: } 5,159,982 \\
\text { 1/14/2016 update: } \\
214,886\end{array}$ \\
\hline $\begin{array}{l}\# 2 \\
\text { Neurological } \\
\text { Outcomes }\end{array}$ & $\begin{array}{l}\text { TITLE-ABS ("nervous system" OR "central-nervous" OR brain OR } \\
\text { blood-brain OR brain-stem OR cerebral OR cortical OR limbic OR } \\
\text { amygdala OR hippocamp* OR hypothalamus OR parahippocamp* OR } \\
\text { perforant OR mesencephalon OR prosencephalon OR rhombencephalon } \\
\text { OR meninges OR dura-matter OR spinal-cord OR ganglia OR ganglion } \\
\text { OR nerve OR nervous OR neural OR neural-tube OR afferent OR } \\
\text { auditory OR olfactory OR oculomotor OR optic OR retinal OR efferent } \\
\text { OR perforant OR neuroglia* OR astrocyte* OR microglia* OR schwann- } \\
\text { cell* OR "myelin sheath" OR neuron* OR axon* OR dendrit* OR } \\
\text { interneuron* OR lewy-bod* OR neurofibril* OR purkinje-cell* OR } \\
\text { pyramidal-cell* OR serotonergic OR neuromuscular OR neuroendocrine }\end{array}$ & $\begin{array}{l}\text { 1/7/2015: 7,558,830 } \\
\text { 1/14/2016 update: } \\
453,341\end{array}$ \\
\hline
\end{tabular}


Systematic Literature Review on the Effects of Fluoride on Learning and Memory in Animal Studies

\begin{tabular}{|c|c|c|}
\hline Search ID & Search Terms & $\begin{array}{l}\text { Date: Number of } \\
\text { Records }\end{array}$ \\
\hline & $\begin{array}{l}\text { OR neurosecret* OR pituitary OR pineal OR synapse* OR synaptic OR } \\
\text { presynaptic OR postsynaptic OR plasticity OR hearing OR vision OR } \\
\text { taste OR smell OR propriocept* OR Nervous system diseases OR } \\
\text { neurologic* OR autonomic-nervous OR central- nervous OR demyelinat* } \\
\text { OR multiple-sclerosis OR myasthenia-gravis OR epilep* OR encephalitis } \\
\text { OR meningitis OR chronic-fatigue OR dyskinesia* OR dyston* OR } \\
\text { parkinson* OR mobility OR motor-control OR motor-abilit* OR motor- } \\
\text { skill* OR cranial-nerve OR huntington* OR tourette* OR ataxia OR } \\
\text { hydranencephaly OR neurofibra* OR tauopath* OR fibromyalgia OR } \\
\text { myopath* OR neurodegenerat* OR neuroimmunomodulat* OR } \\
\text { neuromuscular OR neuropath* OR neurosecret* OR neurotox* OR } \\
\text { neurobehav* OR dyslexi* OR cognitive OR cognition OR intellect* OR } \\
\text { intelligence OR mental- deficienc* OR mental-deficit* OR down- } \\
\text { syndrome OR rett-syndrome OR memory-deficit* OR memory-impair* } \\
\text { OR memory-loss* OR Mental disorders OR Developmental disabilities } \\
\text { OR developmental- delay* OR developmental-disabilit* OR anxiety OR } \\
\text { obsessive- compulsive OR phobia* OR phobic OR antisocial OR } \\
\text { personality OR schizophrenia OR behavior OR paranoid OR paranoia OR } \\
\text { dementia OR senile OR senility OR alzheimer OR learning OR aphasia } \\
\text { OR autism OR autistic OR attention-deficit) }\end{array}$ & \\
\hline $\begin{array}{l}\text { \# } 3 \\
\text { Fluoride }\end{array}$ & $\begin{array}{l}\text { 1/7/2015: TITLE-ABS ( ( fluorid* OR flurid* OR fluorin* OR florin*) } \\
\text { AND NOT ( f-labeled OR fluorine-18 OR imaging OR radioligand* ) } \\
\text { )1/14/2016 update: TITLE-ABS(fluorid* OR flurid* OR fluorin* OR } \\
\text { florin* OR fluorosis) AND NOT (18F OR F-18 OR "fluorine-18" OR } \\
\text { 19F OR F-19 OR "fluorine-19" OR (PET AND scan) OR f-labeled OR } \\
\text { imaging OR radioligand*) AND PUBYEAR>2014 }\end{array}$ & $\begin{array}{l}\text { 1/7/2015: } 153,560 \\
\text { 1/14/2016 update: } \\
7895\end{array}$ \\
\hline
\end{tabular}

\section{A.6. Web of Science}

Search Note: Searched 1/7/2015.

Table A-6. Search Strategy for Web of Science

\begin{tabular}{|c|c|c|}
\hline Search ID & Search Terms & $\begin{array}{l}\text { Date: Number of } \\
\text { Records }\end{array}$ \\
\hline \# 4 & $\begin{array}{l}\# 1 \text { AND \#2 AND \#3 } \\
\text { Indexes=SCI-EXPANDED, SSCI, CPCI-S, CPCI-SSH, BKCI-S, BKCI-SSH, } \\
\text { CCR-EXPANDED, }\end{array}$ & $\begin{array}{l}\text { 1/7/2015: } 1,836 \\
\text { 1/14/2016 update: } 59\end{array}$ \\
\hline $\begin{array}{l}\text { \# } 1 \\
\text { Animal } \\
\text { Studies }\end{array}$ & 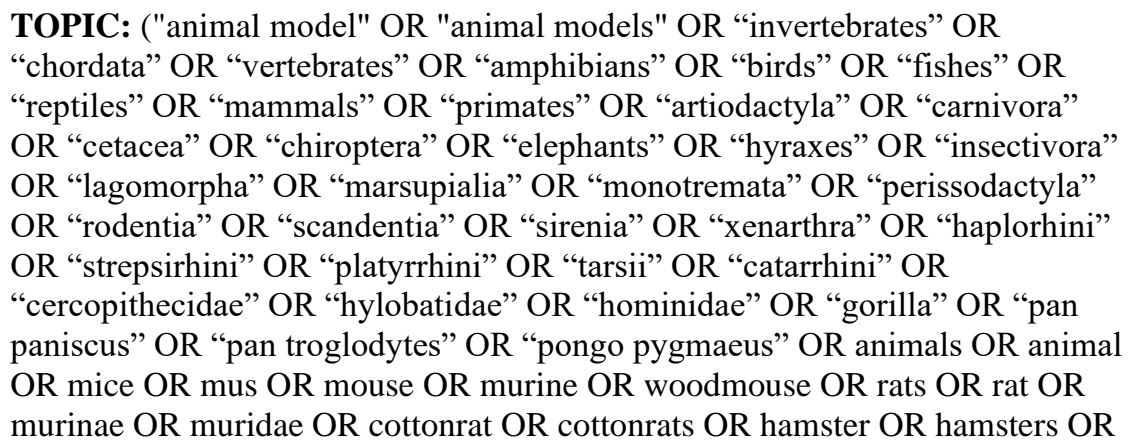 & $\begin{array}{l}\text { 1/7/2015: } 5,945,667 \\
\text { 1/14/2016 update: } \\
248,464\end{array}$ \\
\hline
\end{tabular}


Systematic Literature Review on the Effects of Fluoride on Learning and Memory in Animal Studies

\begin{tabular}{ccc} 
Search ID & Search Terms & $\begin{array}{c}\text { Date: Number of } \\
\text { Records }\end{array}$ \\
\hline
\end{tabular}

cricetinae OR rodentia OR rodent OR rodents OR pigs OR pig OR swine OR swines OR piglets OR piglet OR boar OR boars OR "sus scrofa" OR ferrets OR ferret OR polecat OR polecats OR "mustela putorius" OR "guinea pigs" OR "guinea pig" OR cavia OR callithrix OR marmoset OR marmosets OR cebuella OR hapale OR octodon OR chinchilla OR chinchillas OR gerbillinae OR gerbil OR gerbils OR jird OR jirds OR merione OR meriones OR rabbits OR rabbit OR hares OR hare OR diptera OR flies OR fly OR dipteral OR drosophila OR drosophilidae OR cats OR cat OR carus OR felis OR nematoda OR nematode OR nematodes OR sipunculida OR dogs OR dog OR canine OR canines OR canis OR sheep OR sheeps OR mouflon OR mouflons OR ovis OR goats OR goat OR capra OR capras OR rupicapra OR rupicapras OR chamois OR haplorhini OR monkey OR monkeys OR anthropoidea OR anthropoids OR saguinus OR tamarin OR tamarins OR leontopithecus OR hominidae OR ape OR apes OR "pan paniscus" OR bonobo OR bonobos OR "pan troglodytes" OR gibbon OR gibbons OR siamang OR siamangs OR nomascus OR symphalangus OR chimpanzee OR chimpanzees OR prosimian OR prosimians OR "bush baby" OR bush babies OR galagos OR galago OR pongidae OR gorilla OR gorillas OR "pongo pygmaeus" OR orangutan OR orangutans OR lemur OR lemurs OR lemuridae OR horse OR horses OR equus OR cow OR calf OR bull OR chicken OR chickens OR gallus OR quail OR bird OR birds OR quails OR poultry OR poultries OR fowl OR fowls OR reptile OR reptilia OR reptiles OR snakes OR snake OR lizard OR lizards OR alligator OR alligators OR crocodile OR crocodiles OR turtle OR turtles OR amphibian OR amphibians OR amphibia OR frog OR frogs OR bombina OR salientia OR toad OR toads OR "epidalea calamita" OR salamander OR salamanders OR eel OR eels OR fish OR fishes OR pisces OR catfish OR catfishes OR siluriformes OR arius OR heteropneustes OR sheatfish OR perch OR perches OR percidae OR perca OR trout OR trouts OR char OR chars OR salvelinus OR minnow OR cyprinidae OR carps OR carp OR zebrafish OR zebrafishes OR goldfish OR goldfishes OR guppy OR guppies OR chub OR chubs OR tinca OR barbels OR barbus OR pimephales OR promelas OR "poecilia reticulata" OR mullet OR mullets OR eel OR eels OR seahorse OR seahorses OR mugil curema OR atlantic cod OR shark OR sharks OR catshark OR anguilla OR salmonid OR salmonids OR whitefish OR whitefishes OR salmon OR salmons OR sole OR solea OR lamprey OR lampreys OR pumpkinseed OR sunfish OR sunfishes OR tilapia OR tilapias OR turbot OR turbots OR flatfish OR flatfishes OR sciuridae OR squirrel OR squirrels OR chipmunk OR chipmunks OR suslik OR susliks OR vole $\mathrm{OR}$ voles $\mathrm{OR}$ lemming $\mathrm{OR}$ lemmings $\mathrm{OR}$ muskrat $\mathrm{OR}$ muskrats $\mathrm{OR}$ lemmus OR otter OR otters OR marten OR martens OR martes OR weasel OR badger OR badgers OR ermine OR mink OR minks OR sable OR sables OR gulo OR gulos OR wolverine OR wolverines OR mustela OR llama OR llamas OR alpaca OR alpacas OR camelid OR camelids OR guanaco OR guanacos OR chiroptera OR chiropteras OR bat OR bats OR fox OR foxes OR iguana OR iguanas OR xenopus laevis OR parakeet OR parakeets OR parrot OR parrots OR donkey OR donkeys OR mule OR mules OR zebra OR zebras OR shrew OR shrews OR bison OR bisons OR buffalo OR buffaloes OR deer OR deers OR bear OR bears OR panda OR pandas OR "wild hog" OR "wild boar" OR fitchew OR fitch OR beaver OR beavers OR jerboa OR jerboas OR capybara OR capybaras) Indexes=SCI-EXPANDED, SSCI, CPCI-S, CPCI-SSH, BKCI-S, BKCI-SSH, CCR-EXPANDED, 
Systematic Literature Review on the Effects of Fluoride on Learning and Memory in Animal Studies

\begin{tabular}{|c|c|c|}
\hline Search ID & Search Terms & $\begin{array}{c}\text { Date: Number of } \\
\text { Records }\end{array}$ \\
\hline $\begin{array}{l}\# 2 \\
\text { Neurolog- } \\
\text { ical } \\
\text { outcomes }\end{array}$ & $\begin{array}{l}\text { TOPIC: ("nervous system" OR "central-nervous" OR brain OR blood- brain } \\
\text { OR brain-stem OR cerebral OR cortical OR limbic OR amygdala OR } \\
\text { hippocamp* OR hypothalamus OR parahippocamp* OR perforant OR } \\
\text { mesencephalon OR prosencephalon OR rhombencephalon OR meninges OR } \\
\text { dura-matter OR spinal-cord OR ganglia OR ganglion OR nerve OR nervous OR } \\
\text { neural OR neural-tube OR afferent OR auditory OR olfactory OR oculomotor } \\
\text { OR optic OR retinal OR efferent OR perforant OR neuroglia* OR astrocyte* } \\
\text { OR microglia* OR schwann- cell* OR "myelin sheath" OR neuron* OR axon* } \\
\text { OR dendrit* OR interneuron* OR lewy-bod* OR neurofibril* OR purkinje- } \\
\text { cell* OR pyramidal-cell* OR serotonergic OR neuromuscular OR } \\
\text { neuroendocrine OR neurosecret* OR pituitary OR pineal OR synapse* OR } \\
\text { synaptic OR presynaptic OR postsynaptic OR plasticity OR hearing OR vision } \\
\text { OR taste OR smell OR propriocept* OR Nervous system diseases OR } \\
\text { neurologic* OR autonomic-nervous OR central- nervous OR demyelinat* OR } \\
\text { multiple-sclerosis OR myasthenia-gravis OR epilep* OR encephalitis OR } \\
\text { meningitis OR chronic-fatigue OR dyskinesia* OR dyston* OR parkinson* OR } \\
\text { mobility OR motor-control OR motor-abilit* OR motor-skill* OR cranial-nerve } \\
\text { OR huntington* OR tourette* OR ataxia OR hydranencephaly OR neurofibra* } \\
\text { OR tauopath* OR fibromyalgia OR myopath* OR neurodegenerat* OR } \\
\text { neuroimmunomodulat* OR neuromuscular OR neuropath* OR neurosecret* } \\
\text { OR neurotox* OR neurobehav* OR dyslexi* OR cognitive OR cognition OR } \\
\text { intellect* OR intelligence OR mental- deficienc* OR mental-deficit* OR down- } \\
\text { syndrome OR rett-syndrome OR memory-deficit* OR memory-impair* OR } \\
\text { memory-loss* OR Mental disorders OR Developmental disabilities OR } \\
\text { developmental- delay* OR developmental-disabilit* OR anxiety OR obsessive- } \\
\text { compulsive OR phobia* OR phobic OR antisocial OR personality OR } \\
\text { schizophrenia OR behavior OR paranoid OR paranoia OR dementia OR senile } \\
\text { OR senility OR alzheimer OR learning OR aphasia OR autism OR autistic OR } \\
\text { attention-deficit) Indexes=SCI-EXPANDED, SSCI, CPCI-S, CPCI-SSH, } \\
\text { BKCI-S, BKCI-SSH, CCR-EXPANDED, }\end{array}$ & $\begin{array}{l}\text { 1/7/2015: 7,065,701 } \\
\text { 1/14/2016 update: } \\
419,568\end{array}$ \\
\hline $\begin{array}{l}\text { \# } 3 \\
\text { Fluoride }\end{array}$ & $\begin{array}{l}\text { 1/7/2015: TOPIC: ((fluorid* OR flurid* OR fluorin* OR florin*) NOT (f- } \\
\text { labeled OR "fluorine-18" OR imaging OR radioligand*)) Indexes=SCI- } \\
\text { EXPANDED, SSCI, CPCI-S, CPCI-SSH, BKCI-S, BKCI-SSH, CCR- } \\
\text { EXPANDED1/14/2016 update: TI=(fluorid* OR flurid* OR fluorin* OR } \\
\text { florin* OR fluorosis) NOT TS=((18F OR F-18 OR "fluorine-18" OR 19F OR } \\
\text { F-19 OR "fluorine-19" OR (PET AND scan) OR f-labeled OR imaging OR } \\
\text { radioligand*)) Indexes=SCI-EXPANDED, SSCI, CPCI-S, CPCI-SSH, BKCI-S, } \\
\text { BKCI-SSH, CCR- }\end{array}$ & $\begin{array}{l}\text { 1/7/2015: } 147,851 \\
\text { 1/14/2016 update: } \\
2551\end{array}$ \\
\hline
\end{tabular}


Systematic Literature Review on the Effects of Fluoride on Learning and Memory in Animal Studies

\section{Appendix B. Data Extraction Elements for Animal Studies}

\section{Table B-1. Data Extraction Elements for Animal Studies}

\begin{tabular}{|c|c|}
\hline Data Type & Element \\
\hline \multirow[t]{2}{*}{ Funding } & Funding source(s) \\
\hline & Reporting of COI by authors and/or translators (*reporting bias) \\
\hline \multirow[t]{3}{*}{ Animal Model } & Sex \\
\hline & Species \\
\hline & Strain \\
\hline \multirow[t]{9}{*}{ Treatment } & Chemical name and CAS number \\
\hline & Source of chemical \\
\hline & Purity of chemical (*information bias) \\
\hline & Dose levels or concentration (as presented and converted to $\mathrm{mg} / \mathrm{kg}$ bw/d when possible) \\
\hline & $\begin{array}{l}\text { Other dose-related details, such as whether administered dose level was verified by } \\
\text { measurement, information on internal dosimetry (*information bias) }\end{array}$ \\
\hline & Vehicle used for exposed animals \\
\hline & Route of administration (e.g., oral, inhalation, dermal, injection) \\
\hline & Age or lifestage at start of dosing and at health outcome assessment \\
\hline & $\begin{array}{l}\text { Duration and frequency of dosing (e.g., hours, days, weeks when administration was ended, } \\
\text { days per week) }\end{array}$ \\
\hline \multirow[t]{10}{*}{ Methods } & $\begin{array}{l}\text { Study design (e.g., single treatment, acute, subchronic (e.g., } 90 \text { days in a rodent), chronic, } \\
\text { multigenerational, developmental, other) }\end{array}$ \\
\hline & $\begin{array}{l}\text { Guideline compliance (i.e., use of EPA, OECD, NTP or another guideline for study design, } \\
\text { conducted under GLP guideline conditions, non-GLP but consistent with guideline study, non- } \\
\text { guideline peer-reviewed publication) }\end{array}$ \\
\hline & $\begin{array}{l}\text { Number of animals per group (and dams per group in developmental studies) (*missing data } \\
\text { bias) }\end{array}$ \\
\hline & $\begin{array}{l}\text { Randomization procedure, allocation concealment, blinding during outcome assessment } \\
\text { (*selection bias) }\end{array}$ \\
\hline & Method to control for litter effects in developmental studies (*information bias) \\
\hline & Use of negative controls and whether controls were untreated, vehicle-treated, or both \\
\hline & Endpoint health category (e.g., reproductive) \\
\hline & Endpoint (e.g., infertility) \\
\hline & Diagnostic or method to measure endpoint (*information bias) \\
\hline & Statistical methods (*information bias) \\
\hline
\end{tabular}


Systematic Literature Review on the Effects of Fluoride on Learning and Memory in Animal Studies

\begin{tabular}{ll}
\hline \multicolumn{1}{c}{ Data Type } & \multicolumn{1}{c}{ Element } \\
\hline Results & $\begin{array}{l}\text { Measures of effect at each dose or concentration level (e.g., mean, median, frequency, measures } \\
\text { of precision or variance) or description of qualitative results. When possible, OHAT will } \\
\text { convert measures of effect to a common metric with associated 95\% confidence intervals (CI). } \\
\text { Most often, measures of effect for continuous data will be expressed as percent control } \\
\text { response, mean difference, or standardized mean difference. Categorical data will be expressed } \\
\text { as relative risk (RR, also called risk ratio). } \\
\text { No observed effect level (NOEL), lowest observed effect level (LOEL), benchmark dose } \\
\text { (BMD) analysis, statistical significance of other dose levels, or other estimates of effect } \\
\text { presented in paper. Note: The NOEL and LOEL are highly influenced by study design, give no } \\
\text { quantitative information about the relationship between dose and response, and can be subject } \\
\text { to author's interpretation (e.g., a statistically significant effect might not be considered } \\
\text { biologically important). Also, a NOEL does not necessarily mean zero response. Ideally, the } \\
\text { response rate or effect size at specific dose levels is used as the primary measure to characterize } \\
\text { the response. } \\
\text { If not presented in the study, statistical power can be assessed during data extraction using an } \\
\text { approach that assesses the ability to detect a 10\% to } 20 \% \text { change from control group's response } \\
\text { for continuous data, or a relative risk or odds ratio of 1.5-2 for categorical data, using the } \\
\text { outcome frequency in the control group to determine sample size. Recommended sample sizes } \\
\text { to achieve 80\% power for a given effect size, i.e., 10\% or } 20 \% \text { change from control, will be } \\
\text { compared to sample sizes used in the study to categorize statistical power. Studies will be } \\
\text { considered adequately powered when sample size for } 80 \% \text { power is met. } \\
\text { Observations on dose response (e.g., trend analysis, description of whether dose-response shape } \\
\text { appears to be monotonic, nonmonotonic) } \\
\text { Data on internal concentration, toxicokinetics, or toxicodynamics (when reported) }\end{array}$ \\
$\begin{array}{l}\text { Documentation of author queries, use of digital rulers to estimate data values from figures, } \\
\text { exposure unit, statistical result conversions, etc. }\end{array}$ \\
\hline Other
\end{tabular}




\section{Appendix C. Studies Excluded from the Analysis for Having "Probably High" or "Definitely High" Risk of Bias in Multiple Critical Domains}

Table C-1. Studies Excluded from Analysis for Risk of Bias

\begin{tabular}{|c|c|c|c|c|c|c|}
\hline $\begin{array}{l}\text { Click to See } \\
\text { Risk of Bias } \\
\text { Rationale }\end{array}$ & Study & $\begin{array}{c}\text { No } \\
\text { Randomization }^{1}\end{array}$ & $\begin{array}{c}\text { Concern or Lack } \\
\text { of Information on } \\
\text { Exposure } \\
\text { Characterization }\end{array}$ & $\begin{array}{l}\text { Concern for } \\
\text { Lack of } \\
\text { Blinding } \\
\text { Outcome } \\
\text { Assessment }{ }^{1}\end{array}$ & $\begin{array}{l}\text { Lack of } \\
\text { Control } \\
\text { for Litter } \\
\text { Effects }^{1}\end{array}$ & Other Factors \\
\hline$\underline{1}$ & $\begin{array}{l}\text { Băran- } \\
\text { Poesina et al. } \\
\text { (2013) }\end{array}$ & $\mathrm{X}$ & $\mathrm{X}$ & $X$ & $\mathrm{X}$ & $\begin{array}{l}\text { Were outcome data incomplete due to attrition or exclusion } \\
\text { from analysis? Not reported. } 16 \text { animals per group were } \\
\text { treated but sample size was not reported for any results. Stated } \\
\text { that } 2 \text { pairs of each F1 group were mated to create the F } 2 \\
\text { generation, but no information provided on how many } \\
\text { animals were tested. }\end{array}$ \\
\hline$\underline{2}$ & $\begin{array}{l}\text { Basha and } \\
\text { Sujitha } \\
\text { (2012) }\end{array}$ & $\mathrm{X}$ & $\mathrm{X}$ & $\mathrm{X}$ & & \\
\hline$\underline{3}$ & $\begin{array}{l}\text { Basha et al. } \\
\text { (2011) }\end{array}$ & $\mathrm{X}$ & & $\mathrm{X}$ & $\mathrm{X}$ & $\begin{array}{l}\text { Were outcome data incomplete due to attrition or exclusion } \\
\text { from analysis? Not reported. Stated that } 8 \text { dams in the F0 } \\
\text { generation treated, but no indication of how many F1, F2, or } \\
\text { F3 animals were tested. A few results tables for non-behavior } \\
\text { outcomes state } \mathrm{n}=6 \text {. }\end{array}$ \\
\hline$\underline{4}$ & $\begin{array}{l}\text { Bera et al. } \\
(2007)\end{array}$ & $\mathrm{X}$ & $\mathrm{X}$ & $\mathrm{X}$ & & \\
\hline$\underline{5}$ & $\begin{array}{l}\text { Bhatnagar et } \\
\text { al. (2002) }\end{array}$ & & & & & $\begin{array}{l}\text { Were experimental conditions identical across study groups? } \\
\text { Controls drank deionized, defluorinated water and were } \\
\text { untreated and maintained "isolated in a corner of animal room } \\
\text { for two days...kept undisturbed, and the room was locked for } \\
18 \text { hrs. Just after opening the animal room, all mice were } \\
\text { sacrificed." Similar housing conditions were not reported for } \\
\text { treatment groups, which were described as receiving } \\
\text { "...(NaF) orally, dissolved in water... }\end{array}$ \\
\hline$\underline{6}$ & $\begin{array}{l}\text { Elliott } \\
(1967)\end{array}$ & $\mathrm{X}$ & $\mathrm{X}$ & $\mathrm{X}$ & & \\
\hline
\end{tabular}


Systematic Literature Review on the Effects of Fluoride on Learning and Memory in Animal Studies

\begin{tabular}{|c|c|c|c|c|c|c|}
\hline $\begin{array}{l}\text { Click to See } \\
\text { Risk of Bias } \\
\text { Rationale }\end{array}$ & Study & $\begin{array}{c}\text { No } \\
\text { Randomization }^{1}\end{array}$ & $\begin{array}{c}\text { Concern or Lack } \\
\text { of Information on } \\
\text { Exposure } \\
\text { Characterization }\end{array}$ & $\begin{array}{l}\text { Concern for } \\
\text { Lack of } \\
\text { Blinding } \\
\text { Outcome } \\
\text { Assessment }{ }^{1}\end{array}$ & $\begin{array}{l}\text { Lack of } \\
\text { Control } \\
\text { for Litter } \\
\text { Effects }^{1}\end{array}$ & Other Factors \\
\hline$\underline{7}$ & $\begin{array}{l}\text { Gopal et al. } \\
\text { (2006) }\end{array}$ & $\mathrm{X}$ & $\mathrm{X}$ & & & $\begin{array}{l}\text { Were experimental conditions identical across study groups? } \\
\text { Controls did not receive treatment, and experimental } \\
\text { conditions stated only to be "standard laboratory condition." }\end{array}$ \\
\hline$\underline{8}$ & $\begin{array}{l}\text { Han et al. } \\
\text { (2014) }\end{array}$ & & $\mathrm{X}$ & $\mathrm{X}$ & & $\begin{array}{l}\text { Were there any other potential threats to internal validity? } \\
\text { Absence of expected response in control animals in } \\
\text { exploratory behavior test. }\end{array}$ \\
\hline$\underline{9}$ & $\begin{array}{l}\text { Hong et al. } \\
\text { (2005) }\end{array}$ & & $\mathrm{X}$ & $\mathrm{X}$ & $\mathrm{X}$ & $\begin{array}{l}\text { Were outcome data incomplete due to attrition or exclusion } \\
\text { from analysis? Not reported. } 6 \text { dams treated in each group and } \\
\text { offspring tested at PND30, } 60 \text {, and 90, but number of } \\
\text { offspring assessed not reported. }\end{array}$ \\
\hline \multirow[t]{2}{*}{$\underline{10}$} & $\begin{array}{l}\text { Jain et al. } \\
\text { (2015) }\end{array}$ & & $\mathrm{X}$ & $\mathrm{X}$ & & $\begin{array}{l}\text { Were outcome data incomplete due to attrition or exclusion } \\
\text { from analysis? Not reported and cannot be inferred, as neither } \\
\text { number of treated animals nor animals assessed for behavioral } \\
\text { effects reported. }\end{array}$ \\
\hline & & & & & & $\begin{array}{l}\text { Were there any other potential threats to internal validity? } \\
\text { Statistical analyses were reasonable (one-way ANOVA } \\
\text { followed by Bonferroni post-test), but consideration of } \\
\text { homogeneity of variance not reported. Sex of animals used or } \\
\text { if equal numbers of each sex were used not reported. If both } \\
\text { sexes were used, results were reported for the sexes together. }\end{array}$ \\
\hline$\underline{11}$ & $\begin{array}{l}\text { Jetti et al. } \\
(2016)\end{array}$ & $\mathrm{X}$ & $\mathrm{X}$ & $\mathrm{X}$ & & \\
\hline$\underline{12}$ & Liu (1989) & & $\mathrm{X}$ & $\mathrm{X}$ & $X$ & $\begin{array}{l}\text { Were outcome data incomplete due to attrition or exclusion } \\
\text { from analysis? Unclear. } 150 \text { offspring generated from } 70 \\
\text { dams were divided into } 3 \text { groups (unclear how many dams } \\
\text { became pregnant in each group). Also unclear how many } \\
\text { offspring were tested; results for } 22 \text { offspring reported in } \\
\text { Table } 1 \text { (controls = } 8 \text {; low fluoride }=7 \text {; high fluoride }=7 \text { ). }\end{array}$ \\
\hline
\end{tabular}


Systematic Literature Review on the Effects of Fluoride on Learning and Memory in Animal Studies

\begin{tabular}{|c|c|c|c|c|c|c|}
\hline $\begin{array}{l}\text { Click to See } \\
\text { Risk of Bias } \\
\text { Rationale }\end{array}$ & Study & $\begin{array}{c}\text { No } \\
\text { Randomization }^{1}\end{array}$ & $\begin{array}{c}\text { Concern or Lack } \\
\text { of Information on } \\
\text { Exposure } \\
\text { Characterization }\end{array}$ & $\begin{array}{l}\text { Concern for } \\
\text { Lack of } \\
\text { Blinding } \\
\text { Outcome } \\
\text { Assessment }\end{array}$ & $\begin{array}{l}\text { Lack of } \\
\text { Control } \\
\text { for Litter } \\
\text { Effects }^{1}\end{array}$ & Other Factors \\
\hline \multirow[t]{2}{*}{$\underline{13}$} & $\begin{array}{l}\text { Mullenix et } \\
\text { al. (1995) }\end{array}$ & & $\mathrm{X}$ & & $\mathrm{X}$ & $\begin{array}{l}\text { Were outcome data incomplete due to attrition or exclusion } \\
\text { from analysis? Death occurred in about half the weanlings } \\
\text { exposed to } 175 \mathrm{ppm} \text {. Unclear how much attrition in other } \\
\text { treatments. Not all numbers reported and in some cases they } \\
\text { do not sum to numbers of controls or treated animals. }\end{array}$ \\
\hline & & & & & & $\begin{array}{l}\text { Were all measured outcomes reported? Unclear. Results } \\
\text { appear most complete for animals whose treatment started at } \\
\text { weaning or adulthood. Data presentation for RS statistic and } \\
\text { K functions difficult to follow. K scores appear presented for } \\
\text { only certain behavior clusters. }\end{array}$ \\
\hline$\underline{14}$ & $\begin{array}{l}\text { Niu et al. } \\
(2009)\end{array}$ & $\mathrm{X}$ & & $\mathrm{X}$ & $\mathrm{X}$ & \\
\hline$\underline{15}$ & $\begin{array}{l}\text { Niu et al. } \\
\text { (2014) }\end{array}$ & $\mathrm{X}$ & $\mathrm{X}$ & & $\mathrm{X}$ & \\
\hline \multirow[t]{3}{*}{$\underline{16}$} & $\begin{array}{l}\text { Rumiantsev } \\
\text { et al. (1988) }\end{array}$ & $\mathrm{X}$ & & $\mathrm{X}$ & & $\begin{array}{l}\text { Were experimental conditions identical across study groups? } \\
\text { Very few details on experimental conditions provided and } \\
\text { whether control group was based on inhalation or oral } \\
\text { administration unclear, as chemicals administered by different } \\
\text { routes of administration but presented in a table with a single } \\
\text { control group. }\end{array}$ \\
\hline & & & & & & $\begin{array}{l}\text { Were outcome data incomplete due to attrition or exclusion } \\
\text { from analysis? Not reported and cannot be inferred, as neither } \\
\text { number of treated animals nor animals assessed for behavioral } \\
\text { effects reported. }\end{array}$ \\
\hline & & & & & & $\begin{array}{l}\text { Were all measured outcomes reported? Very little description } \\
\text { in methods of procedure, sample sizes not reported, unclear } \\
\text { whether SD or SE is reported in the results table. Were there } \\
\text { any other potential threats to internal validity? Statistical } \\
\text { methods not reported. }\end{array}$ \\
\hline$\underline{17}$ & $\begin{array}{l}\text { Wu et al. } \\
\text { (2008) }\end{array}$ & $\mathrm{X}$ & $\mathrm{X}$ & $\mathrm{X}$ & $\mathrm{X}$ & $\begin{array}{l}\text { Were outcome data incomplete due to attrition or exclusion } \\
\text { from analysis? Number of dams treated not clearly reported } \\
\text { for all groups. Authors report that } 6 \text { of } 18 \text { litters in high dose }\end{array}$ \\
\hline
\end{tabular}


Systematic Literature Review on the Effects of Fluoride on Learning and Memory in Animal Studies

\begin{tabular}{c|c|c|c|c|c|}
\hline $\begin{array}{c}\text { Click to See } \\
\text { Risk of Bias } \\
\text { Rationale }\end{array}$ & Study & $\begin{array}{c}\text { No } \\
\text { Randomization }\end{array}$ & $\begin{array}{c}\text { Concern or Lack } \\
\text { of Information on } \\
\text { Exposure } \\
\text { Characterization }\end{array}$ & $\begin{array}{c}\text { Concern for } \\
\text { Lack of } \\
\text { Blinding } \\
\text { Outcome } \\
\text { Assessment }^{1}\end{array}$ & $\begin{array}{c}\text { Lack of } \\
\text { Control } \\
\text { for Litter } \\
\text { Effects }^{1}\end{array}$ \\
\hline
\end{tabular}

group lost all pups due to no or low milk production. Table 2

reports results for 9-11 litters in each group. Considered to pose potential risk of bias because number of litters varies across groups and number of treated dams not reported in all groups and loss of pups in the high dose group. Results appear to be obtained on 40 pups in each group, but unclear for all outcomes.

Were all measured outcomes reported? Unclear. Description of methods too incomplete to evaluate "Behavioral teratology testing of the offspring rats was conducted according to procedures outlined in the literature."

Were there any other potential threats to internal validity? Statistical methods not reported.

\begin{tabular}{|c|c|c|c|c|c|}
\hline$\underline{18}$ & $\begin{array}{l}\text { Zhang et al. } \\
\text { (2009) }\end{array}$ & $\mathrm{X}$ & $\mathrm{X}$ & $X$ & $\begin{array}{l}\text { Were outcome data incomplete due to attrition or exclusion } \\
\text { from analysis? Unclear. One dam treated per group, resulting } \\
\text { in } 37 \mathrm{~F} 1 \text { offspring (numbers range from } 9-10 \text { per group). } \\
\text { Sample size in results not presented but no loss of animals } \\
\text { reported. }\end{array}$ \\
\hline$\underline{19}$ & $\begin{array}{l}\text { Zhang et al. } \\
\text { (2001) }\end{array}$ & $X$ & $X$ & & $\begin{array}{l}\text { Were outcome data incomplete due to attrition or exclusion } \\
\text { from analysis? Unclear. Authors did not report in the methods } \\
\text { section number of mice treated and inferring is difficult } \\
\text { because the number of mice among treatment and control } \\
\text { groups differed and number of mice reported under same } \\
\text { treatment group differed between Table } 1 \text { and Table } 2 \text {. }\end{array}$ \\
\hline
\end{tabular}




\section{Appendix D. Learning and Memory: Morris Water Maze (All Studies)}

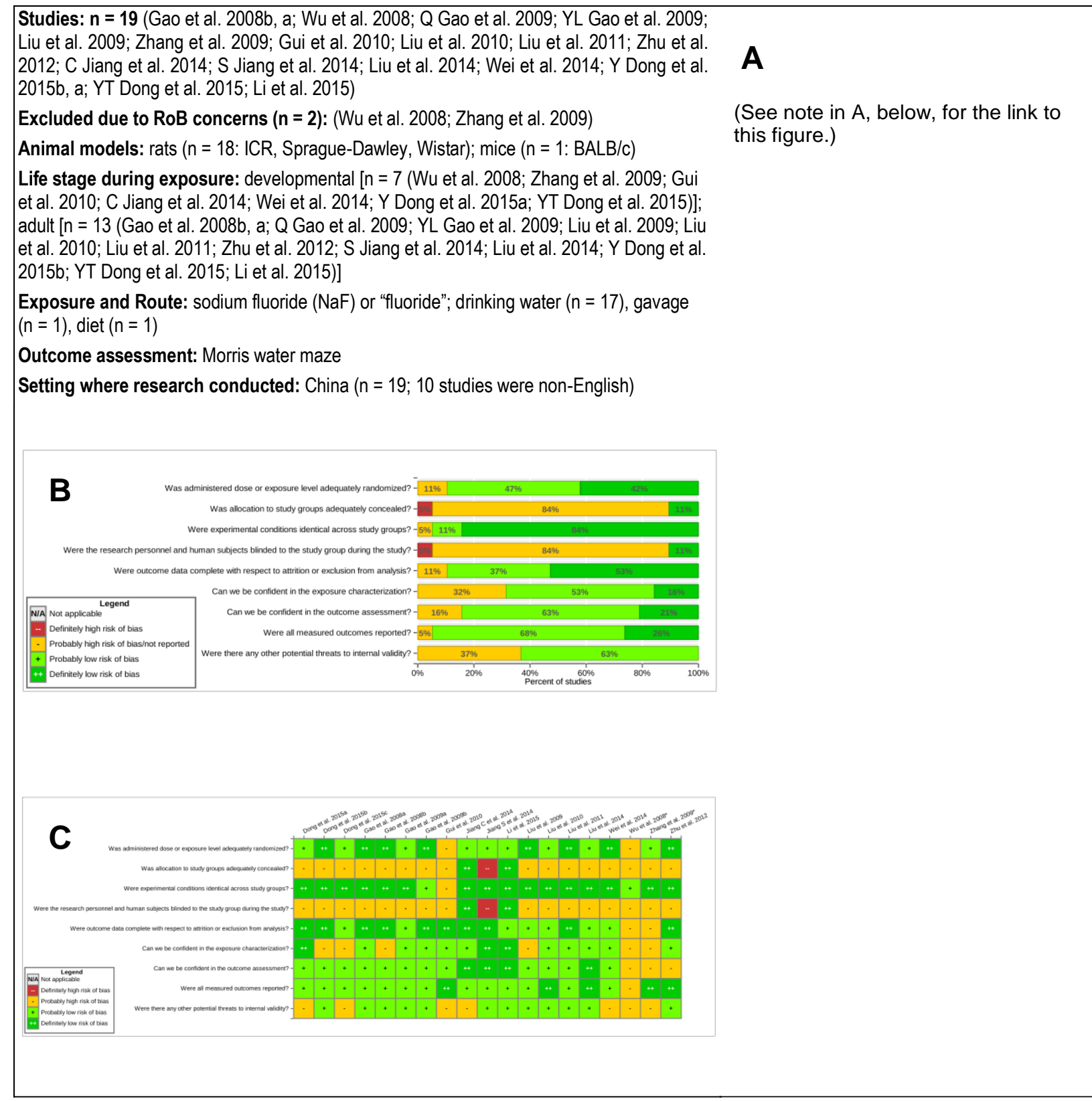

Figure D-1. Learning and Memory: Morris Water Maze (All Studies)

(A) Descriptive features and results of studies expressed as percent change from control response. Click to see interactive graphic sorted by study. Note: Some data from Liu et al. (2009) are also presented in Liu et al. (2011) (escape time, acquisition, day 6). Findings are presented under Liu et al. (2009) in the figure. Apparently, identical data were reported in Dong et al. (2015a); Dong et al. (2015b) and Dong et al. (2015c). Only data from Dong et al. (2015a) is presented in figure. (B) Risk of bias across studies. (C) Risk of bias assessments for individual studies (*study excluded from primary analysis due to concerns for risk of bias). 


\section{Appendix E. Learning and Memory: T-Maze}

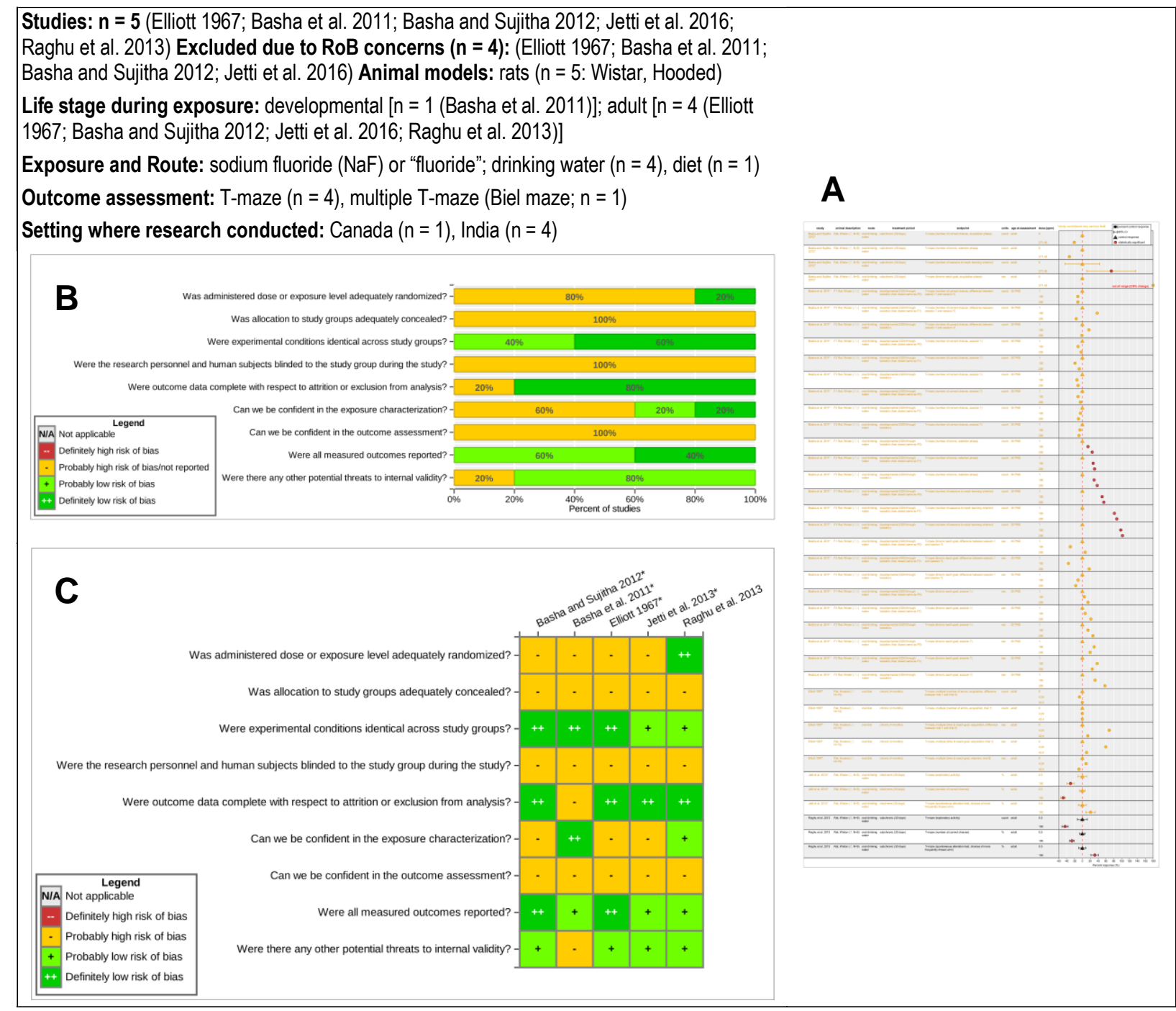

Figure E-1. Learning and Memory: T-Maze

(A) Descriptive features and results of studies expressed as percent change from control response. Click to see interactive graphic sorted by study. (B) Risk of bias across studies. (C) Risk of bias assessments for individual studies (*study excluded from primary analysis due to concerns for risk of bias). 


\section{Appendix F. Learning and Memory: Y-Maze}

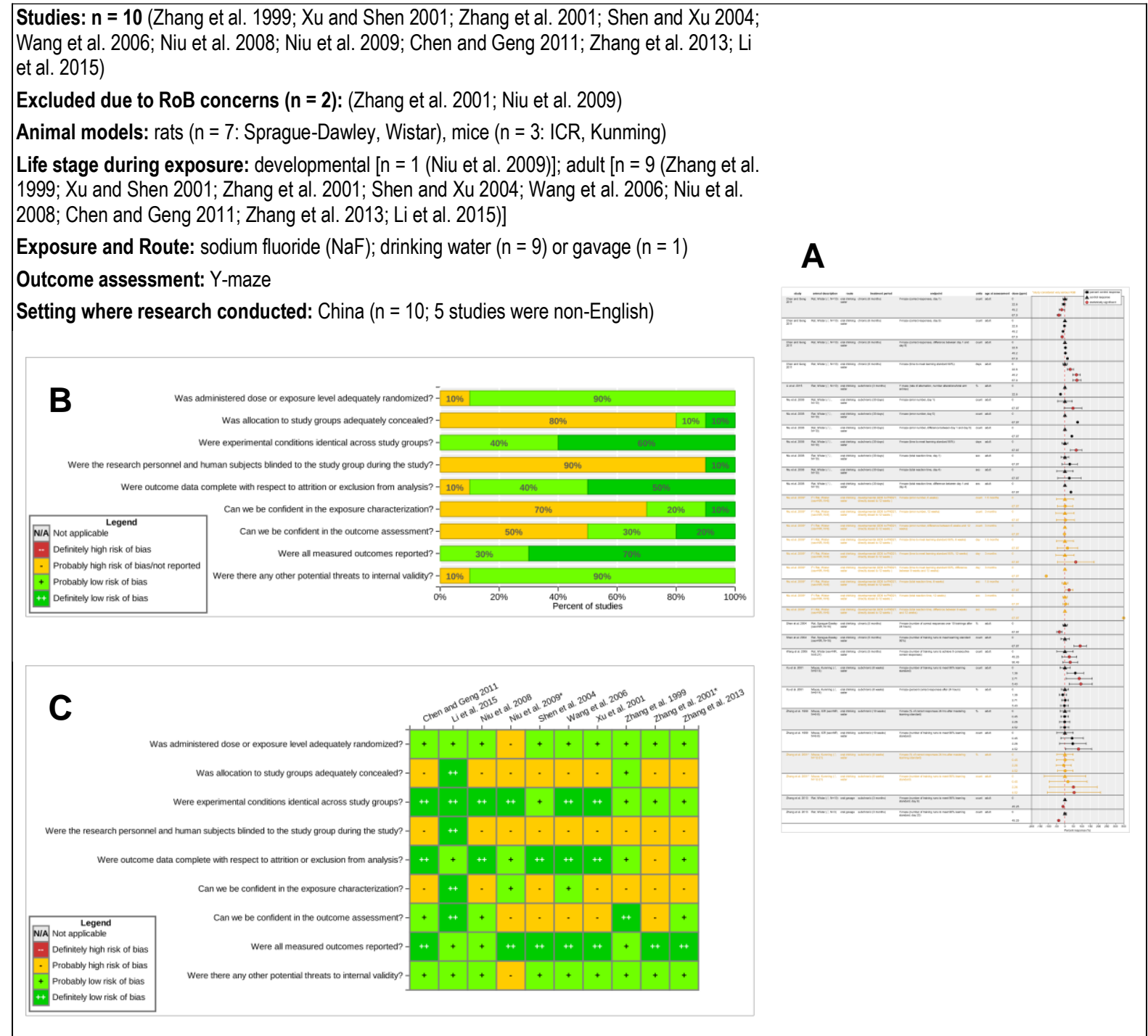

Figure F-1. Learning and Memory: Y-Maze

(A) Descriptive features and results of studies expressed as percent change from control response. Click to see interactive graphic sorted by study. (B) Risk of bias across studies. (C) Risk of bias assessments for individual studies (*study excluded from primary analysis due to concerns for risk of bias). 


\section{Appendix G. Learning and Memory: Other Maze Tests}

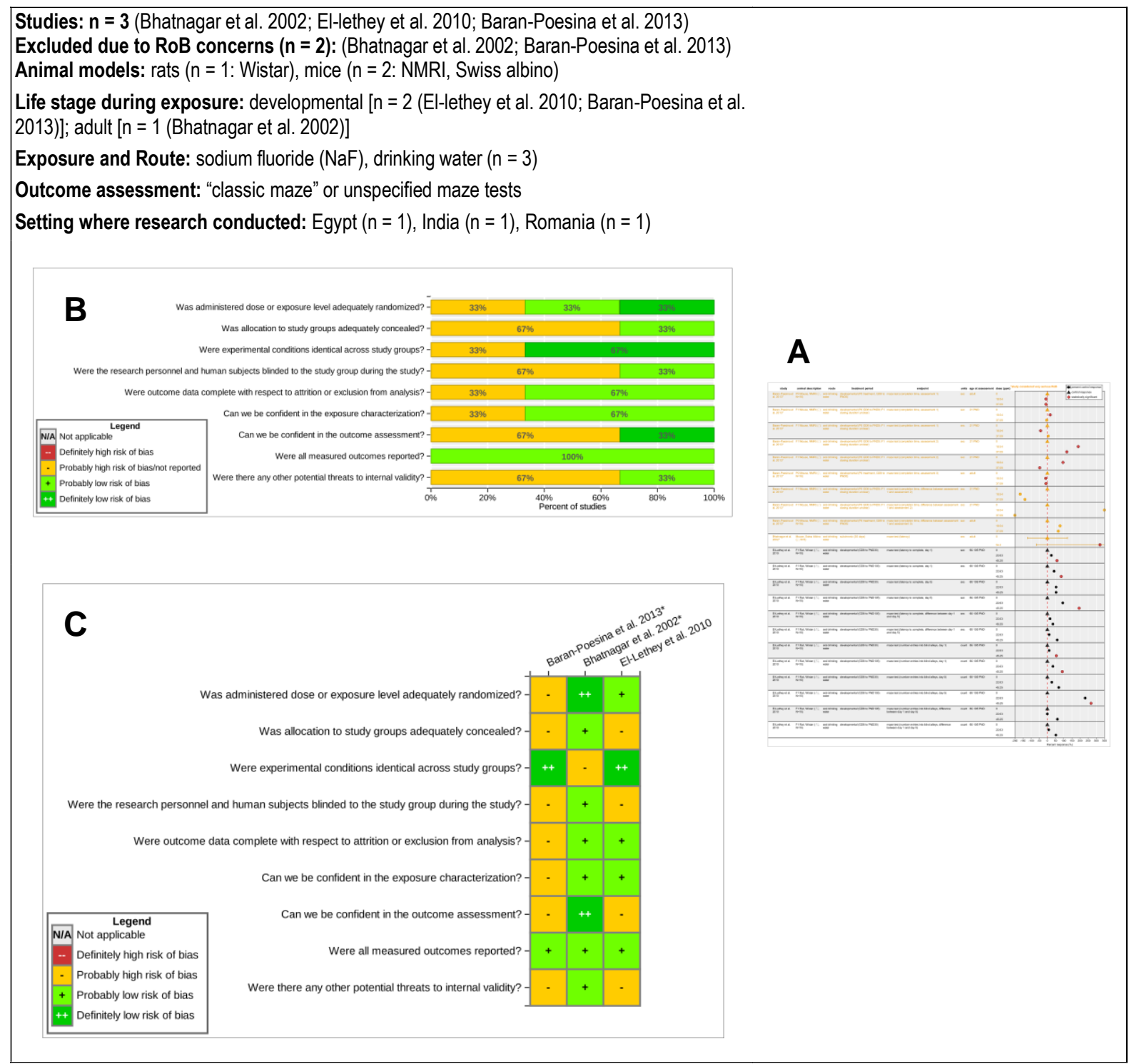

Figure G-1. Learning and Memory: Other Maze Tests

(A) Descriptive features and results of studies expressed as percent change from control response. Click to see interactive graphic sorted by study. Note: For Bhatnagar et al. (2002), data presented only for control and high dose groups (0-, 3.68-, 7.37-, and 14.72-ppm dose groups treated). (B) Risk of bias across studies. (C) Risk of bias assessments for individual studies (*study excluded from primary analysis due to concerns for risk of bias). 


\section{Appendix H. Learning and Memory: Exploratory Behavior}

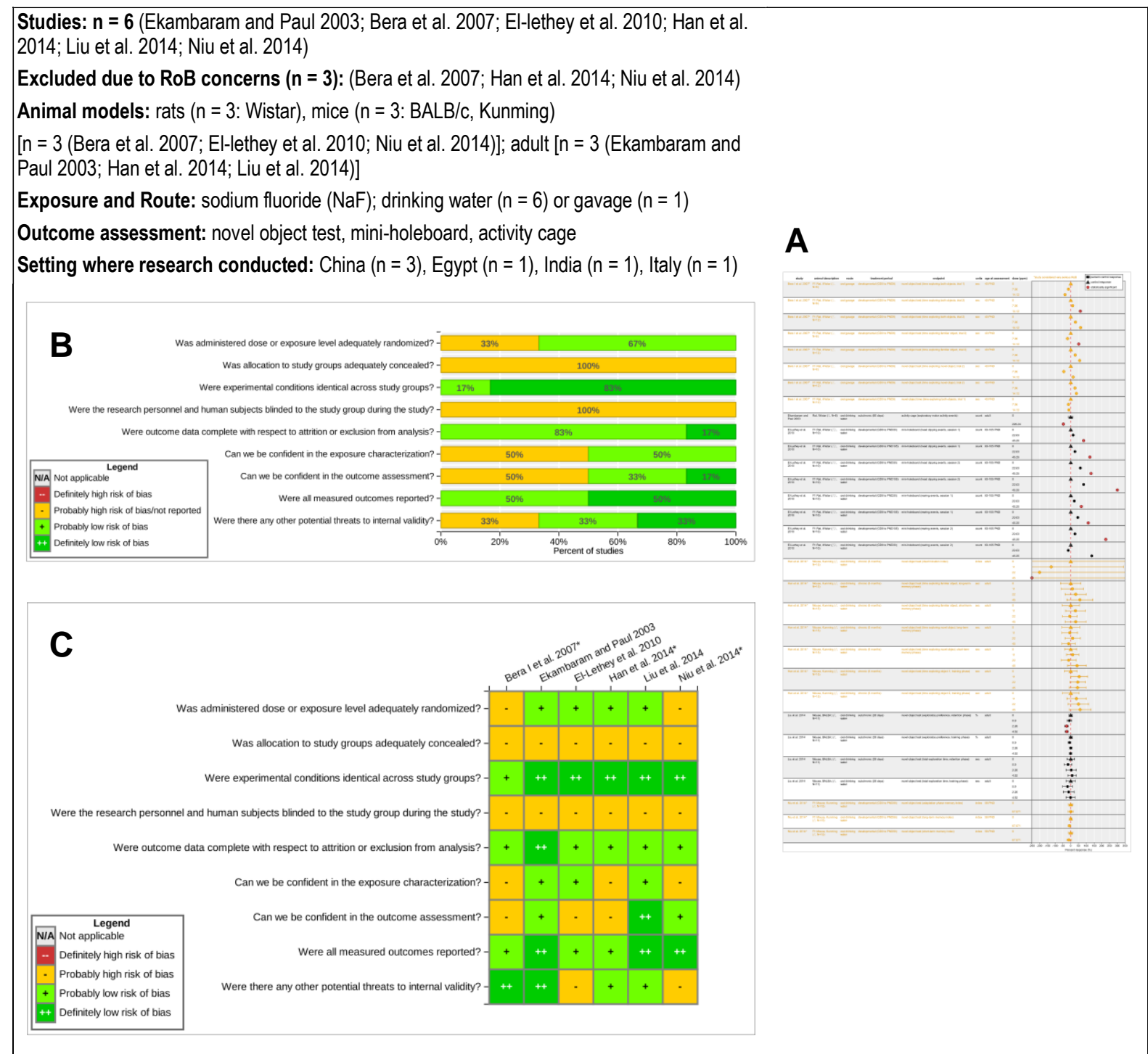

Figure H-1. Learning and Memory: Exploratory Behavior

(A) Descriptive features and results of studies expressed as percent change from control response. Click to see interactive graphic sorted by study. (B) Risk of bias across studies. (C) Risk of bias assessments for individual studies (*study excluded from primary analysis due to concerns for risk of bias). 


\section{Appendix I. Learning and Memory: Passive Avoidance}

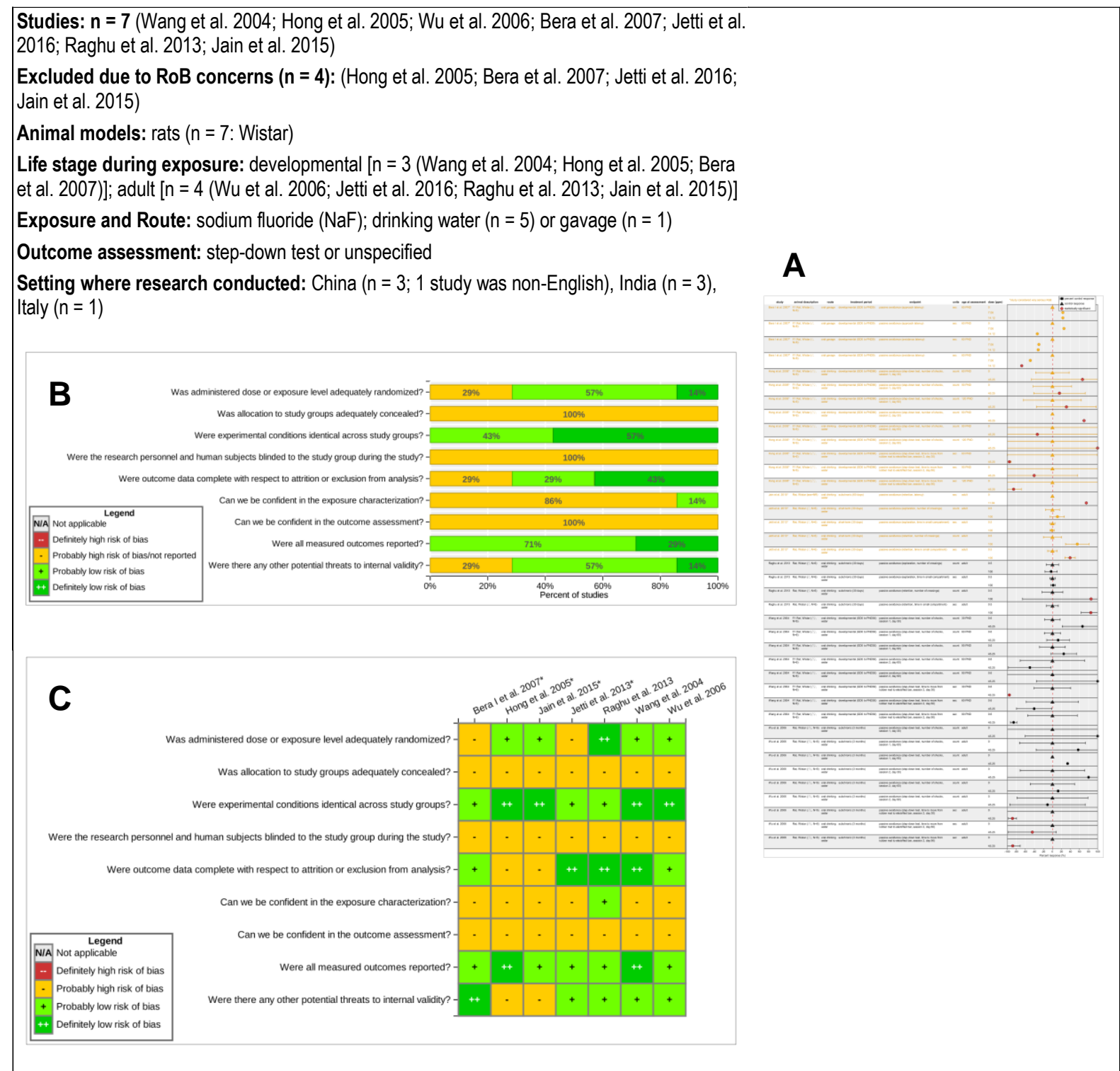

Figure I-1. Learning and Memory: Passive Avoidance

(A) Descriptive features and results of studies expressed as percent change from control response. Click to see interactive graphic sorted by study. Note: For Wang et al. (2004), percent control response value for passive avoidance (step-down test, number of shocks, session 2, day 30) could not be calculated due to a zero response (count) for the control. (B) Risk of bias across studies. (C) Risk of bias assessments for individual studies (*study excluded from primary analysis due to concerns for risk of bias). 


\section{Appendix J. Learning and Memory: Active Avoidance}

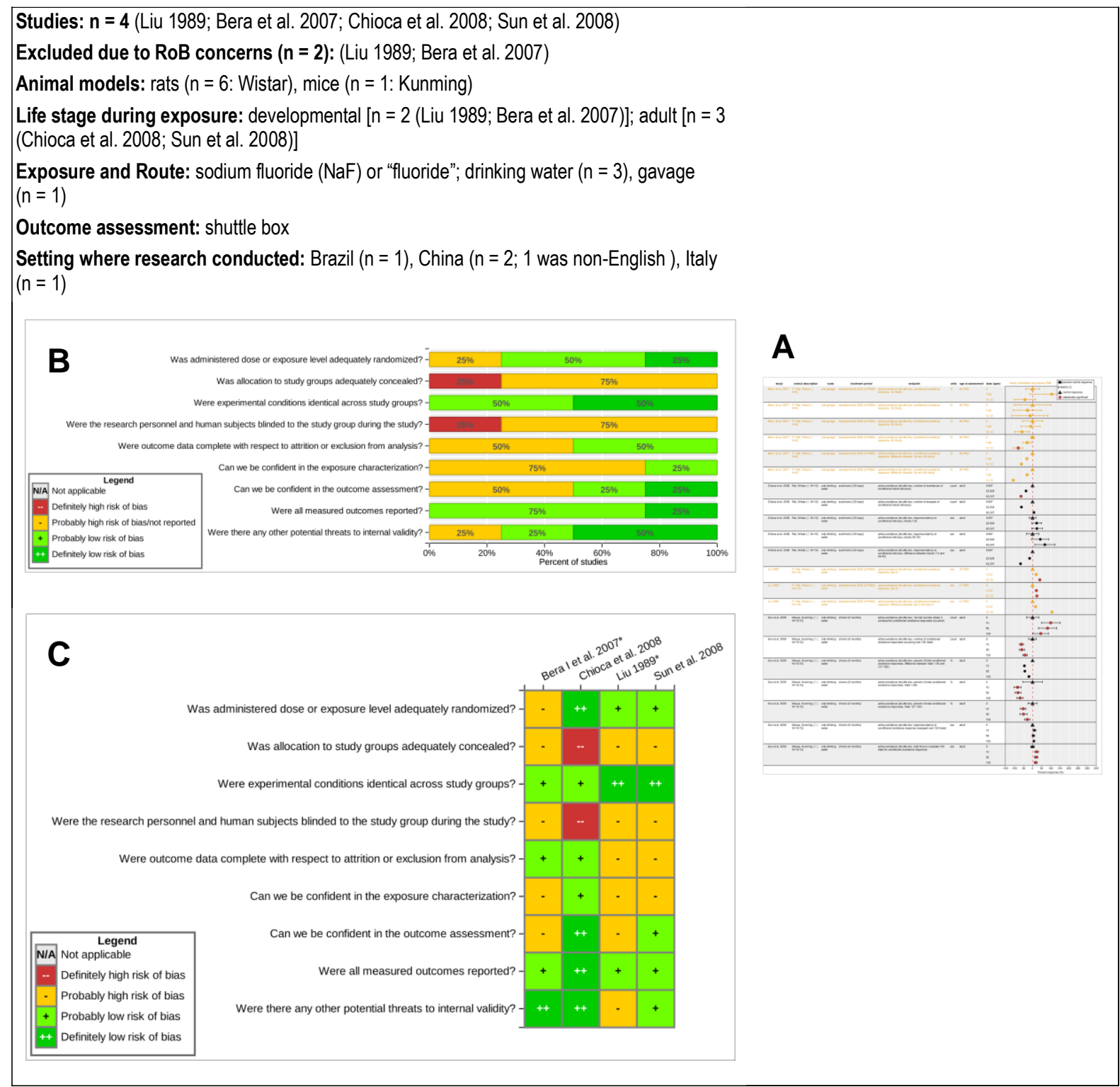

Figure J-1. Learning and Memory: Active Avoidance

(A) Descriptive features and results of studies expressed as percent change from control response. Click to see interactive graphic sorted by study. Note: Data from Wang et al. (2004) for active avoidance (step-down test, number of shocks, session 2, day 30) could not be plotted as percent change from control because the response and SD were 0 in the control group. A significant increase in the number of shocks was observed in the 45-ppm treatment group. (B) Risk of bias across studies. (C) Risk of bias assessments for individual studies (*study excluded from primary analysis due to concerns for risk of bias). 


\section{Appendix K. Motor and Sensory Function: Locomotor Activity}

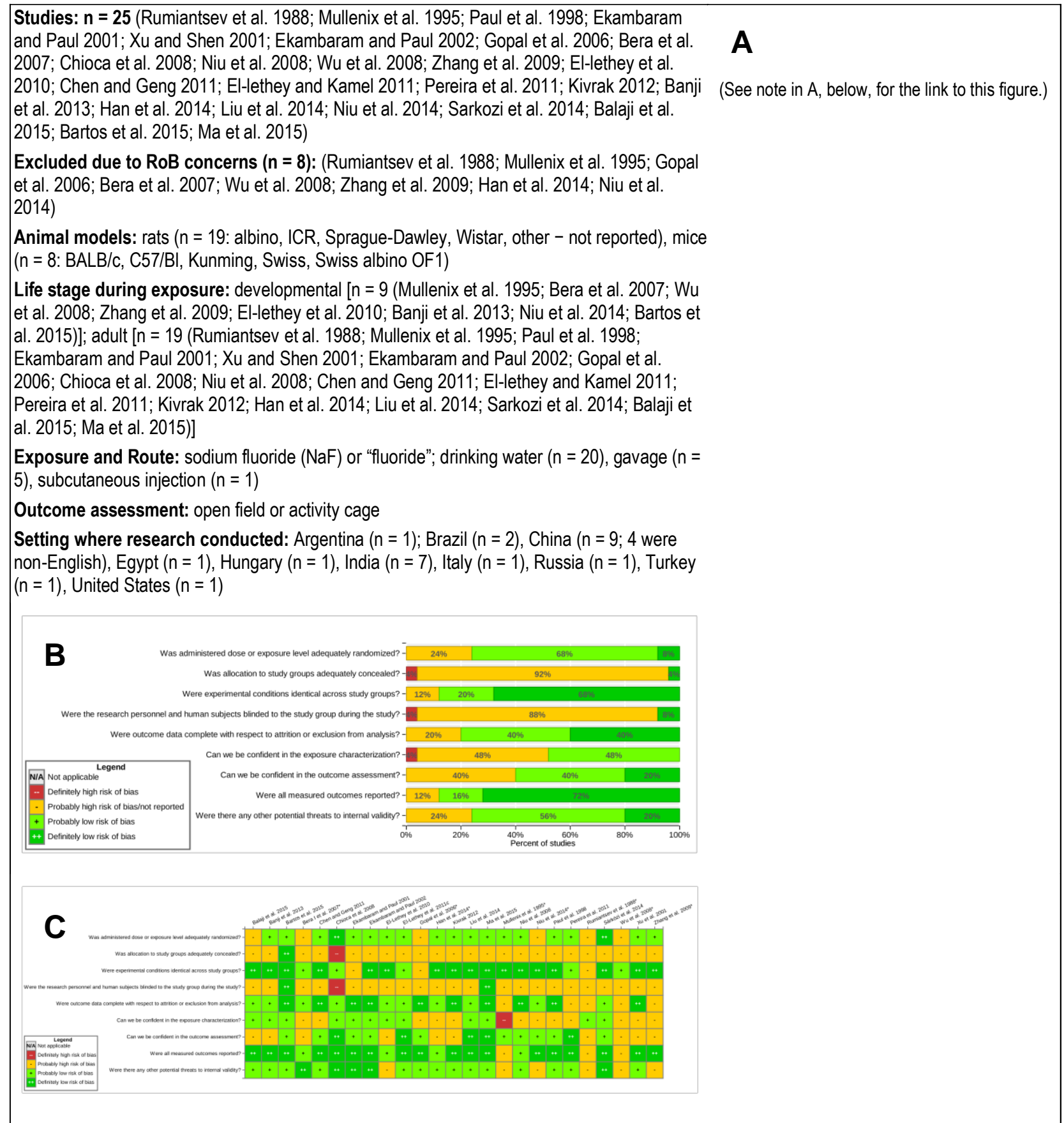

Figure K-1. Motor and Sensory Function: Locomotor Activity

(A) Descriptive features and results of studies expressed as percent change from control response. Click the following link to see interactive graphic sorted by study. Note: Findings for Bera et al. (2007) are not shown because a single conclusion on "locomotor activity" was reported (no significant effects, data not shown). (B) Risk of bias across studies. (C) $\underline{\text { Risk of bias }}$ assessments for individual studies (*study excluded from primary analysis due to concerns for risk of bias). 


\section{Appendix L. Motor and Sensory Function: Movement Coordination}

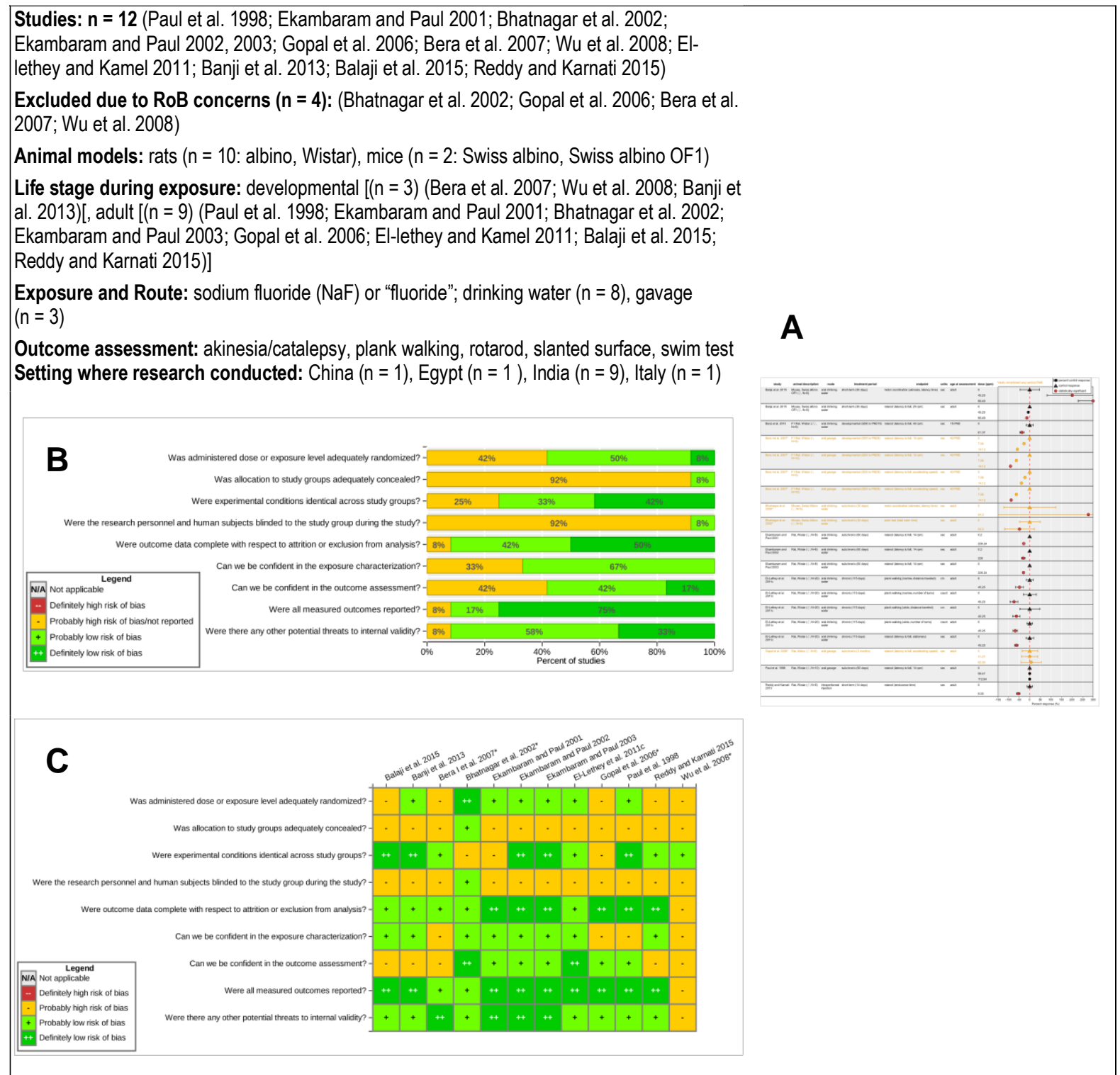

Figure L-1. Motor and Sensory Function: Movement Coordination

(A) Descriptive features and results of studies expressed as percent change from control response. Click to see interactive graphic sorted by study. Note: For Bhatnagar et al. (2002), data presented in paper only for control and high dose groups (0-, 3.68-, 7.37-, and 14.72-ppm dose groups were tested) and data for (catalepsy, latency time) are not included in figure because control values were not presented in paper (findings described as "a significant high value was observed in treated animals $(7.0+/-3.3)$." The following endpoints from Wu et al. (2008) were described as not affected (data not shown): swim test, slanted surface, forelimb suspension. (B) Risk of bias across studies. (C) Risk of bias assessments for individual studies (*study excluded from primary analysis due to concerns for risk of bias). 


\section{Appendix M. Motor and Sensory Function: Reflex and Motor Sensory Development}

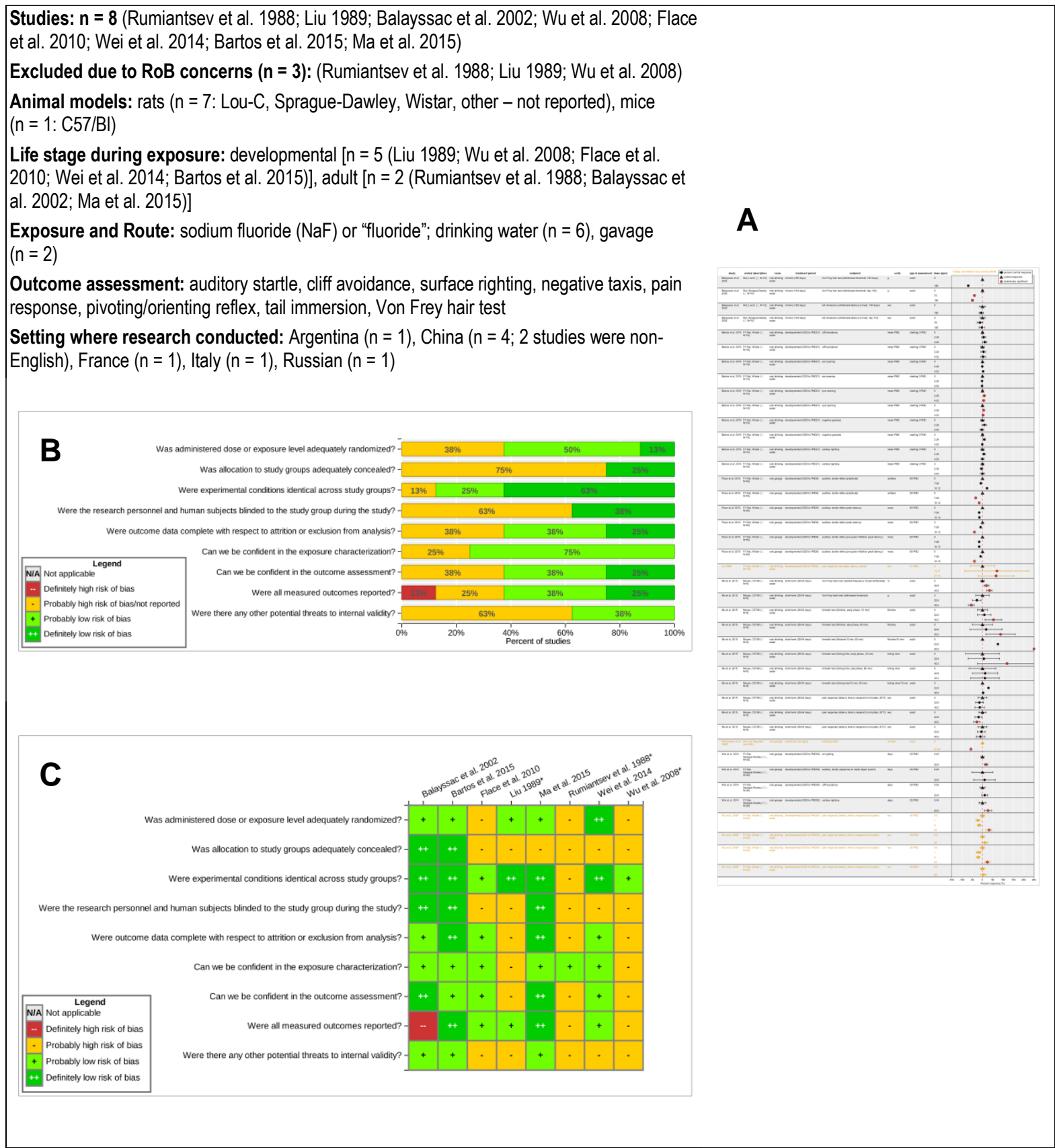

Figure M-1. Motor and Sensory Function: Reflex and Motor Sensory Development

(A) Descriptive features and results of studies expressed as percent change from control response. Click to see interactive graphic sorted by study. Note: The following endpoints from Wu et al. (2008) were described as not affected (data not shown): auditory startle, cliff avoidance, negative taxis, pivoting task, surface righting. (B) Risk of bias across studies. (C) $\underline{\text { Risk of bias }}$ assessments for individual studies (*study excluded from primary analysis due to concerns for risk of bias). 
Systematic Literature Review on the Effects of Fluoride on Learning and Memory in Animal Studies

\section{Appendix N. Anxiety}

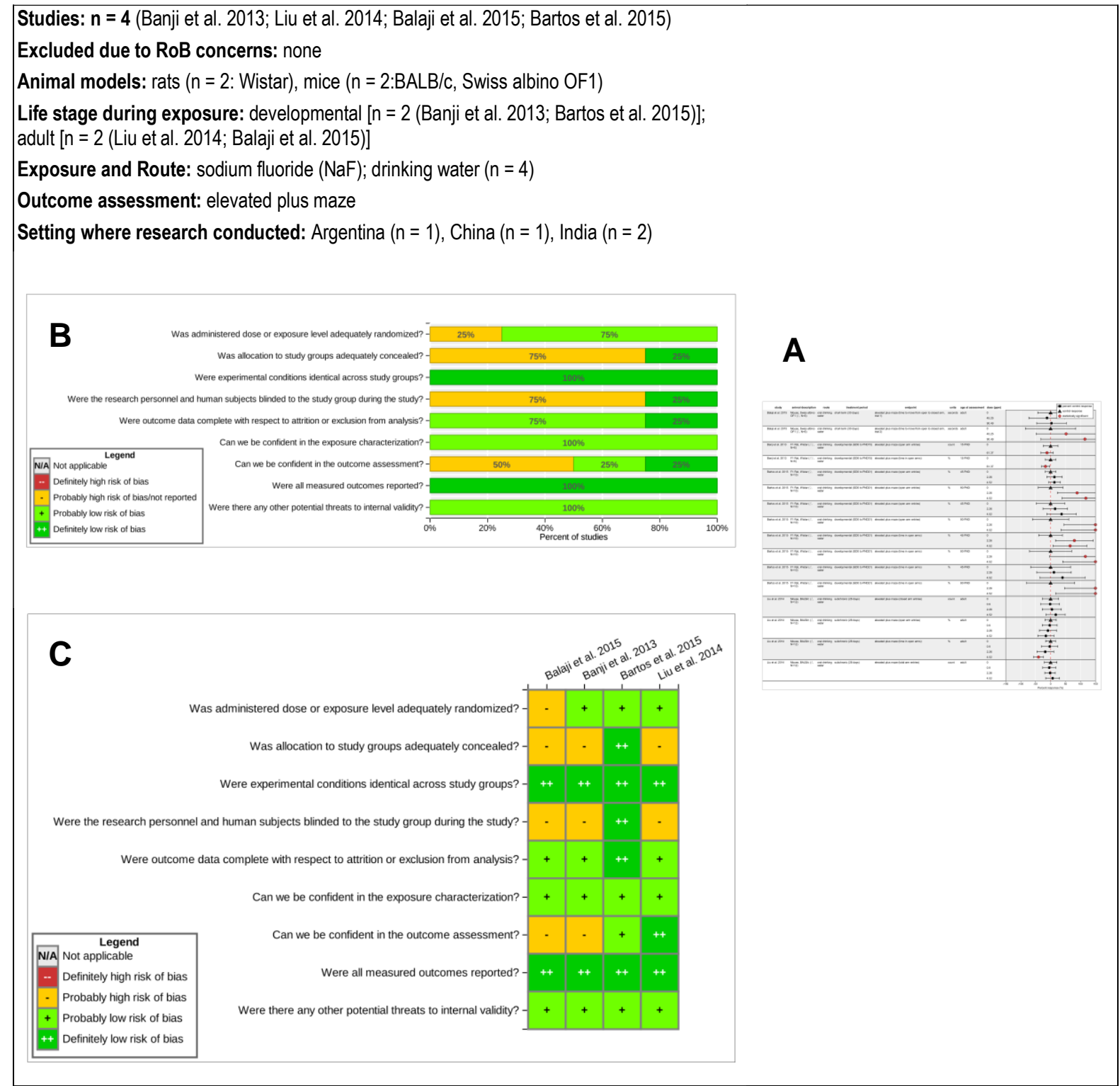

Figure N-1. Anxiety

(A) Descriptive features and results of studies expressed as percent change from control response. Click to see interactive graphic sorted by study. (B) Risk of bias across studies. (C) Risk of bias assessments for individual studies (*study excluded from primary analysis due to concerns for risk of bias). 


\section{Appendix O. Grooming, Defecation, Urination}

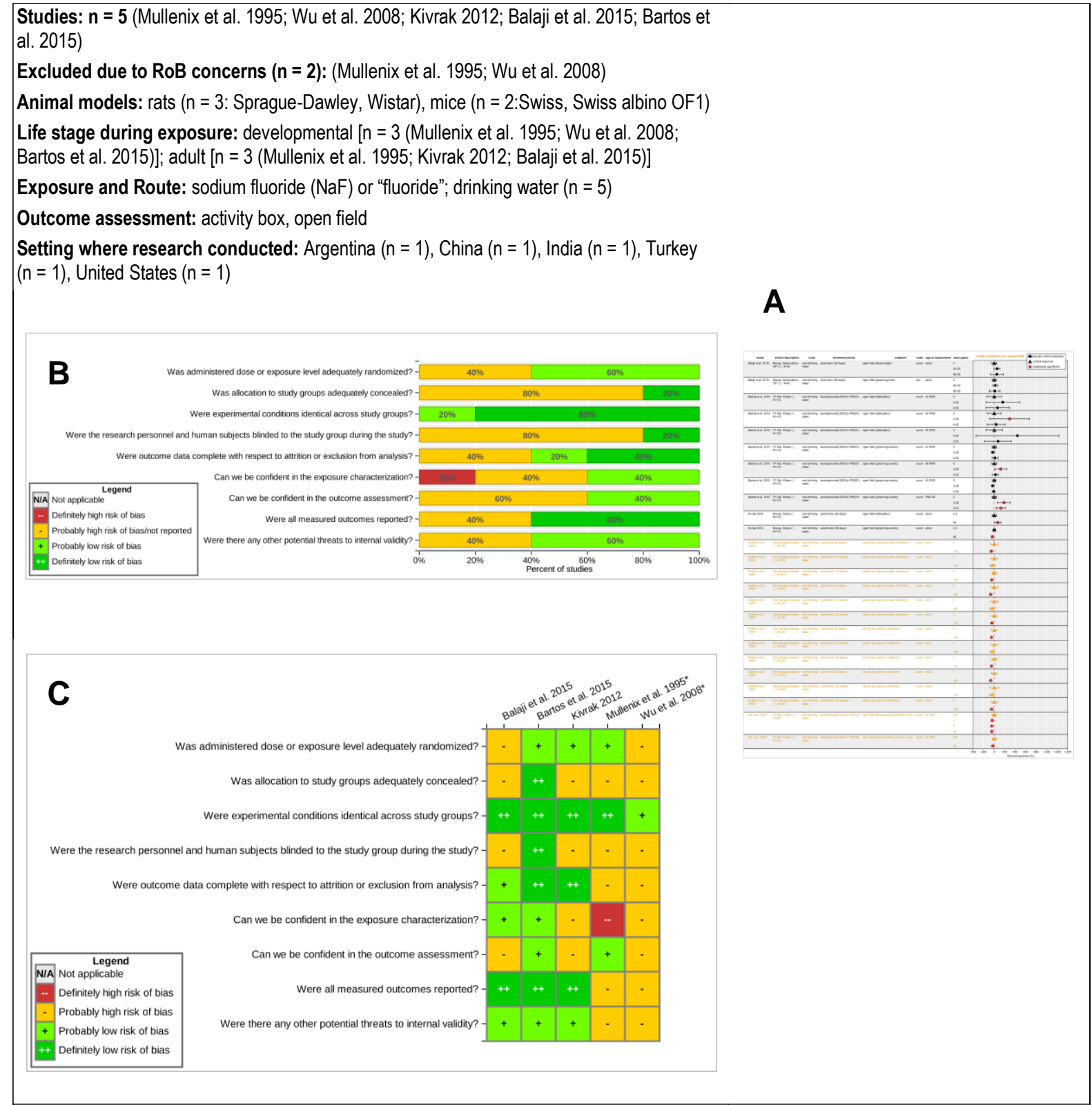

Figure O-1. Grooming, Defecation, Urination

(A) Descriptive features and results of studies expressed as percent change from control response. Click to see interactive graphic sorted by study. Note: The following endpoint from Wu et al. (2008) was described as not affected (data not shown): defecation and urination in open field test. (B) Risk of bias across studies. (C) Risk of bias assessments for individual studies (*study excluded from primary analysis due to concerns for risk of bias). 


\section{Appendix P. Sexual Behavior}

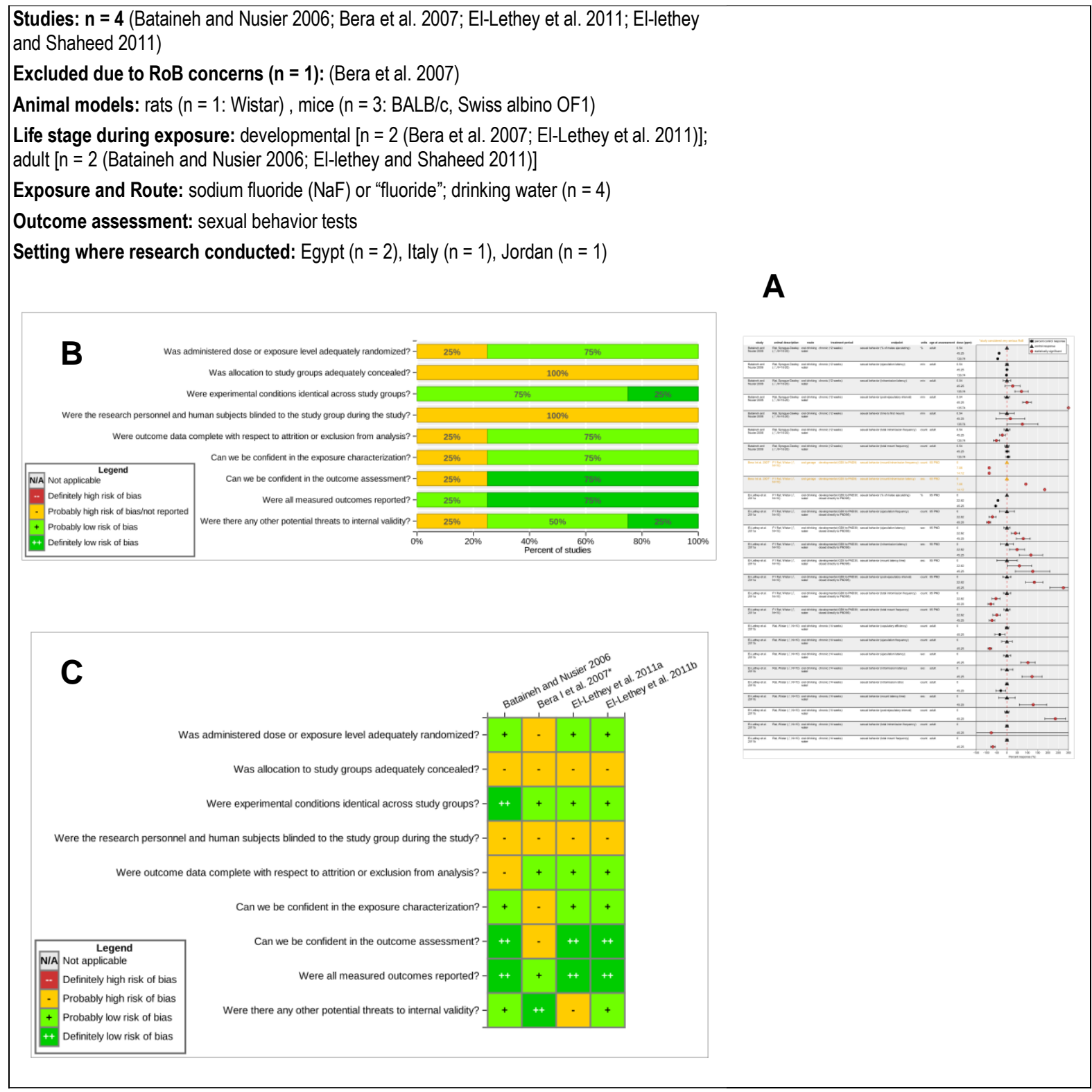

Figure P-1. Sexual Behavior

(A) Descriptive features and results of studies expressed as percent change from control response. Click to see interactive graphic sorted by study. (B) Risk of bias across studies. (C) Risk of bias assessments for individual studies (*study excluded from primary analysis due to concerns for risk of bias). 


\section{Appendix Q. Territorial Aggression}

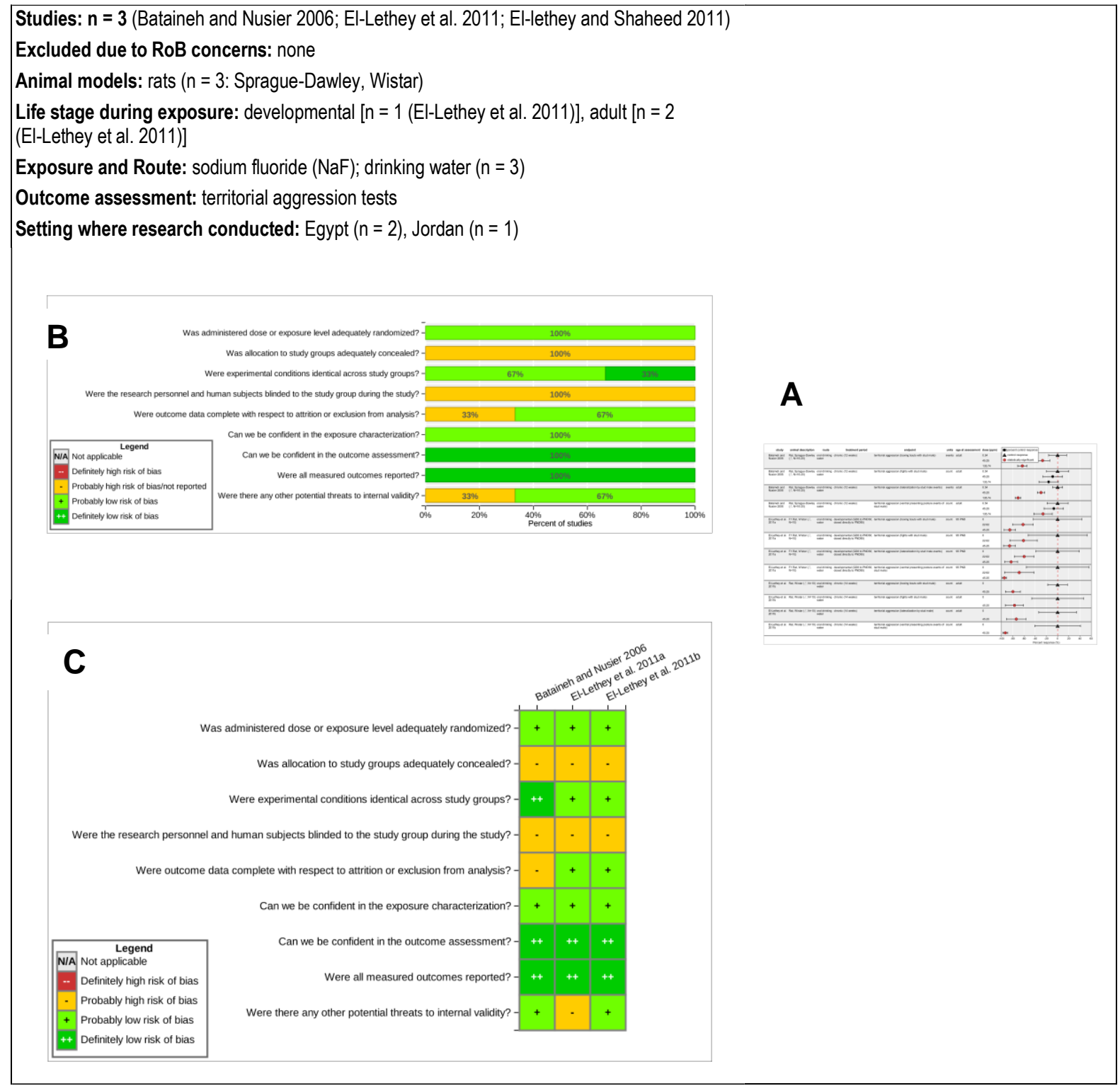

Figure Q-1. Territorial Aggression

(A) Descriptive features and results of studies expressed as percent change from control response. Click to see interactive graphic sorted by study. (B) Risk of bias across studies. (C) Risk of bias assessments for individual studies (*study excluded from primary analysis due to concerns for risk of bias). 
Systematic Literature Review on the Effects of Fluoride on Learning and Memory in Animal Studies

\section{Appendix R. Other}

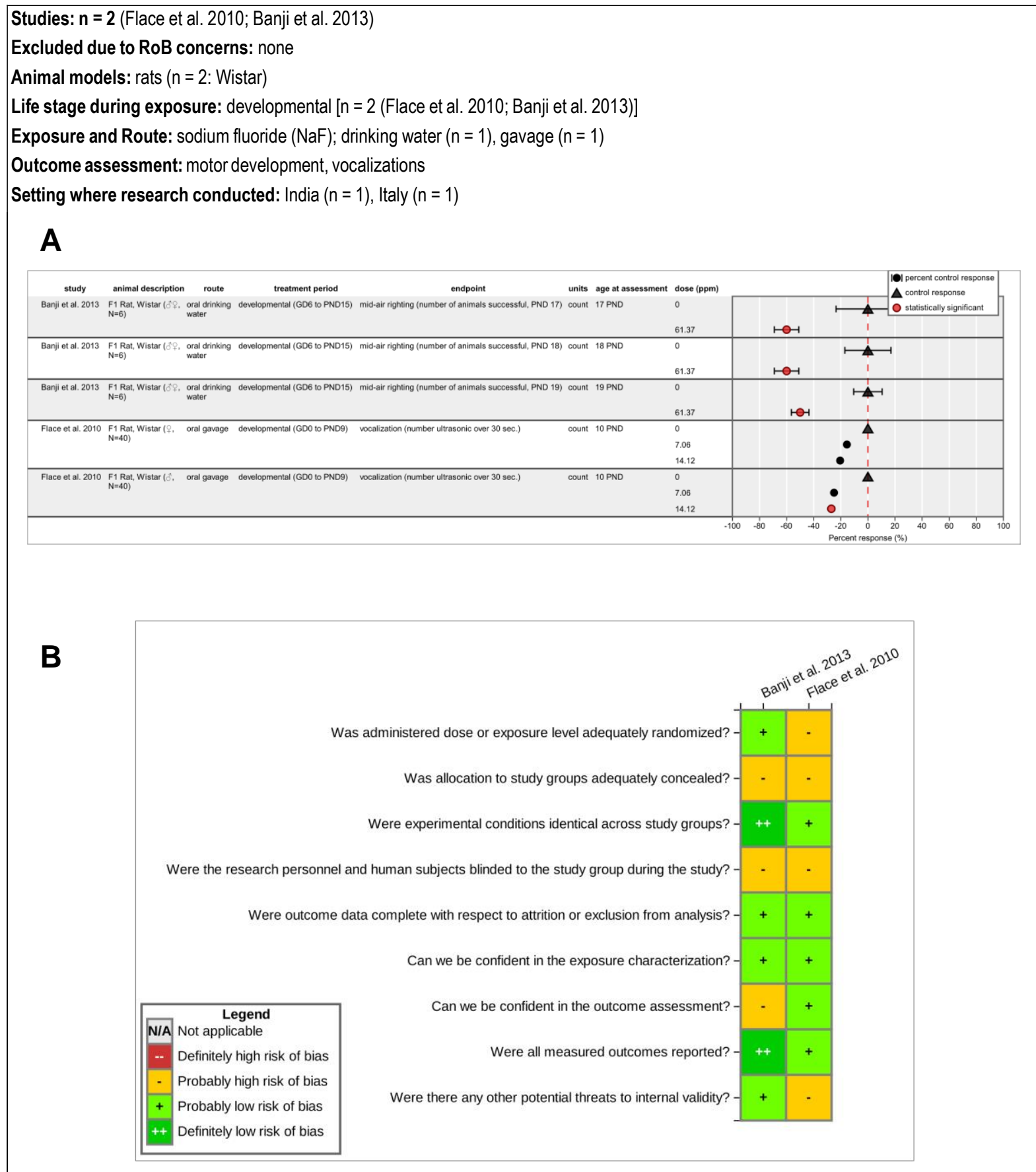

Figure R-1. Other

(A) Descriptive features and results of studies expressed as percent change from control response. Click to see interactive graphic sorted by study. (B) Risk of bias assessments for individual studies (*study excluded from primary analysis due to concerns for risk of bias). 


\section{Appendix S. Fluoride Administered and Converted Doses (Individual Studies)}

Table S-1. Summary of Fluoride Administered and Converted Doses (Individual Studies)

\begin{tabular}{|c|c|c|}
\hline Study & $\begin{array}{l}\text { Fluoride Concentration } \\
\text { ppm }\end{array}$ & $\begin{array}{l}\text { Fluoride Concentration }{ }^{1} \\
\mathrm{mg} / \mathrm{kg}-\mathrm{d}\end{array}$ \\
\hline \multicolumn{3}{|l|}{ Oral Drinking Water } \\
\hline Balaji et al. (2015) & $0,45.3,90.5$ & $0,12.3,24.5$ \\
\hline Balayssac et al. (2002) & $\begin{array}{l}0,150(\text { Lou/C rat }) \\
0,75,150(\text { SD rat })\end{array}$ & $\begin{array}{c}0,20.8 \text { (Lou/C rat) } \\
0,10.4,20.8 \text { (SD rat) }\end{array}$ \\
\hline Banji et al. (2013) & $0,61.4(\mathrm{~F} 1)$ & $0,9.1(\mathrm{~F} 1)$ \\
\hline Băran-Poesina et al. (2013) & $0,18.5,37.1(\mathrm{P} 0, \mathrm{~F} 1, \mathrm{~F} 2)$ & $0,5,10(\mathrm{P} 0, \mathrm{~F} 1, \mathrm{~F} 2)$ \\
\hline Bartos et al. (2015) & $0,2.3,4.5(\mathrm{~F} 1)$ & $0,0.27,0.54(\mathrm{~F} 1)$ \\
\hline Basha and Sujitha (2012) & $0,271.5$ & 0,40 \\
\hline Basha et al. (2011) & 1, 100, $200(\mathrm{~F} 1, \mathrm{~F} 2, \mathrm{~F} 3)$ & $0.16,16.03,32.1(\mathrm{~F} 1, \mathrm{~F} 2, \mathrm{~F} 3)$ \\
\hline Bataineh and Nusier (2006) & $0.54,45.3,135.7$ & $0.08,6.3,18.8$ \\
\hline Bhatnagar et al. (2002) & $0,54.3$ & $0,14.7$ \\
\hline Chen and Geng (2011) & $0,22.6,45.2,67.9$ & $0,3.3,6.7,10$ \\
\hline Chioca et al. (2008) & $0.7,22.6,45.2$ & $0.045,2.33,4.87$ \\
\hline $\begin{array}{l}\text { Dong et al. (2015a); Dong et al. (2015b); } \\
\text { Dong et al. (2015c) }\end{array}$ & $0.5,22.6(\mathrm{~F} 1)$ & $0.07,3.3(\mathrm{~F} 1)$ \\
\hline Ekambaram and Paul (2003) & $0.2,226$ & $0.03,36.3$ \\
\hline Ekambaram and Paul (2002) & $0.2,226$ & $0.04,40.4$ \\
\hline Ekambaram and Paul (2003) & 0,226 & $0,110.7$ \\
\hline El-lethey et al. (2010) & $0,22.6,45.3(\mathrm{~F} 1)$ & $0,2.3,4.9(\mathrm{~F} 1)$ \\
\hline El-lethey et al. (2011) & $0,22.6,45.3(\mathrm{~F} 1)$ & $0,3.1,6.3(\mathrm{~F} 1)$ \\
\hline El-lethey and Shaheed (2011) & $0,45.3$ & $0,6.7$ \\
\hline El-lethey and Kamel (2011) & $0,45.3$ & $0,6.7$ \\
\hline Gao et al. (2008a) & $1,2.3,22.6$ & $0.14,0.33,3.3$ \\
\hline Gao et al. (2008b) & $0.5,2.3,22.6$ & $0.07,0.33,3.3$ \\
\hline Gao et al. (2009a) & $0.23,2.7,22.6$ & $0.03,0.33,3.3$ \\
\hline Han et al. (2014) & $0,11,22,45$ & $0,2.8,5.6,11.5$ \\
\hline Hong et al. (2005) & $0,45.3(\mathrm{~F} 1)$ & $0,6.9(\mathrm{~F} 1)$ \\
\hline Jain et al. (2015) & $0,11.8$ & $0,1.8$ \\
\hline Jetti et al. (2016) & $0.5,100$ & $0.07,14.8$ \\
\hline Jiang et al. (2014a) & $0.3,11.3,22.6,45.3(\mathrm{~F} 1)$ & $0.05,1.6,3.3,6.5(\mathrm{~F} 1)$ \\
\hline Jiang et al. (2014b) & $0,54.3$ & $0,7.5$ \\
\hline Kivrak (2012) & $0.3,40$ & $0.07,9.6$ \\
\hline
\end{tabular}


Systematic Literature Review on the Effects of Fluoride on Learning and Memory in Animal Studies

\begin{tabular}{|c|c|c|}
\hline Study & $\begin{array}{c}\text { Fluoride Concentration }^{1} \\
\text { ppm }\end{array}$ & $\begin{array}{c}\text { Fluoride Concentration }^{1} \\
\mathrm{mg} / \mathrm{kg}-\mathrm{d}\end{array}$ \\
\hline Li et al. (2015) & $0,22.6$ & $0,3.3$ \\
\hline Liu (1989) & $0,13.6,27.2(\mathrm{~F} 1)$ & $0,2.2,4.4(\mathrm{~F} 1)$ \\
\hline Liu et al. (2009) & $0.23,2.3,22.6$ & $0.03,0.3,3.3$ \\
\hline Liu et al. (2010) & $0.23,2.3,22.6$ & $0.03,0.3,3.3$ \\
\hline Liu et al. (2011) & $0.5,2.3,22.6$ & $0.07,0.3,3.3$ \\
\hline Liu et al. (2014) & $0,0.9,2.3,4.5$ & $0,0.23,0.58,1.2$ \\
\hline Ma et al. (2015) & $0,22.6,45.2$ & $0,5.79,11.6$ \\
\hline Mullenix et al. (1995) (6-wk exposure) & 0,100 & $0,21.46$ \\
\hline Mullenix et al. (1995) (16-wk exposure) & 0,125 & 0,19 \\
\hline Niu et al. (2008) & $0,67.9$ & $0,10.4$ \\
\hline Niu et al. (2009) & $0,67.9(\mathrm{~F} 1)$ & $0,10.9(\mathrm{~F} 1)$ \\
\hline Niu et al. (2014) & $0,67.9(\mathrm{~F} 1)$ & $0,18.4(\mathrm{~F} 1)$ \\
\hline Pereira et al. (2011) & $0.7,45.2$ & $0.1,6.7$ \\
\hline Raghu et al. (2013) & $0.5,100$ & $0.07,14.8$ \\
\hline Shen et al. (2004) & $0,67.9$ & $0,9.8$ \\
\hline Sun et al. (2008) & $0,10,50,100$ & $0,2.6,13.1,26.3$ \\
\hline Wang et al. (2004) & $0.6,45.3(\mathrm{~F} 1)$ & $0.01,6.9(\mathrm{~F} 1)$ \\
\hline Wang et al. (2006) & $0,45.3,90.5$ & $0,6.9,13.8$ \\
\hline \multicolumn{3}{|l|}{ Wei et al. (2014) } \\
\hline Whitford et al. (2009) & $0,33.2,85.6,155.2$ & $0,3,6,12$ \\
\hline Wu et al. (2006) & $0,45.3$ & $0,5.8$ \\
\hline Wu et al. (2008) & $\begin{array}{c}0.6,25(\mathrm{~F} 1 \text { postnatal }) \\
0.6,1,5,25(\mathrm{~F} 1 \text { prenatal and } \\
\text { postnatal })\end{array}$ & $\begin{array}{c}0.1,5.3(\mathrm{~F} 1 \text { postnatal }) \\
0.1,0.2,1.1,5.3(\mathrm{~F} 1 \text { prenatal and } \\
\text { postnatal) }\end{array}$ \\
\hline Xu et al. (2001) & $0,1.4,2.7,5.4$ & $0,0.4,0.7,1.4$ \\
\hline Zhang et al. (1999) & $0,0.5,2.3,4.5$ & $0,0.1,0.6,1.2$ \\
\hline Zhang et al. (2001) & $0,0.5,2.3,4.5$ & $0,0.1,0.6,1.2$ \\
\hline Zhang et al. (2009) & $0,1.1,2.3,4.5(\mathrm{~F} 1)$ & $0,0.18,0.36,0.71(\mathrm{~F} 1)$ \\
\hline Zhu et al. (2012) & $0,2.3,4.5,9.05$ & $0,0.34,0.68,1.4$ \\
\hline \multicolumn{3}{|l|}{ Oral diet } \\
\hline Elliott (1967) & $0,4.2,42.4$ & $0,0.61,6.2$ \\
\hline Gui et al. (2010) & $1.8,29.8(\mathrm{~F} 1)$ & $0.26,4.3(\mathrm{~F} 1)$ \\
\hline \multicolumn{3}{|l|}{ Gavage } \\
\hline Bera et al. (2007) & $0,7.1,14.1(\mathrm{~F} 1)$ & $0,1.1,2.3(\mathrm{~F} 1)$ \\
\hline Flace et al. (2010) & $0,7.1,14.1(\mathrm{~F} 1)$ & $0,1.1,2.3(\mathrm{~F} 1)$ \\
\hline Gao et al. (2009a) & $0,62.7$ & $0,9.05$ \\
\hline
\end{tabular}


Systematic Literature Review on the Effects of Fluoride on Learning and Memory in Animal Studies

\begin{tabular}{|c|c|c|}
\hline Study & $\begin{array}{c}\text { Fluoride Concentration }^{1} \\
\text { ppm }\end{array}$ & $\begin{array}{c}\text { Fluoride Concentration }^{1} \\
\text { mg/kg-d }\end{array}$ \\
\hline Gopal et al. (2006) & $0,31.3,62.6$ & $0,4.52,9.05$ \\
\hline Paul et al. (1998) & $0,56.5,112.9$ & $0,9.05,18.1$ \\
\hline Rumiantsev et al. (1988) & $0,33.3$ & 0,5 \\
\hline Sarkozi et al. (2015) & $0,14.8$ & $0,2.3$ \\
\hline Wei et al. (2014) & $0.5,22.6(\mathrm{~F} 1)$ & $0.07,3.44(\mathrm{~F} 1)$ \\
\hline Zhang et al. (2013) & $0,45.3$ & $0,6.7$ \\
\hline \multicolumn{3}{|c|}{$\begin{array}{l}\text { Subcutaneous or Intraperitoneal } \\
\text { Injection }\end{array}$} \\
\hline Mullenix et al. (1995) & - & $\begin{array}{c}0,0.06 \mathrm{mg} / \mathrm{kg}(\mathrm{P} 0) \\
(9 \text { prenatal injections })\end{array}$ \\
\hline Reddy and Karnati (2015) & - & $0,9.05 \mathrm{mg} / \mathrm{kg}$ bw (single injection) \\
\hline \multicolumn{3}{|c|}{$\begin{array}{l}{ }^{1} \text { In general, the administered chemical in studies is sodium fluoride }(\mathrm{NaF}) \text {, and not all studies provide } \mathrm{F} \text { equivalents. Dose levels } \\
\text { are presented here as average daily doses of fluoride }(\mathrm{mg} / \mathrm{kg}-\mathrm{d}) \text { and equivalent water concentrations of fluoride }(\mathrm{ppm}) \text {. As } \\
\text { needed, dose conversions were made using US EPA }(1988 ; 1994) \text { default food or water consumption rates and body weights } \\
\text { (using subchronic age and experiment duration) for the species/strain and sex of the animal of interest. Dose levels in mg/kg-d } \\
\text { can vary for a given ppm across different studies if the studies use different species/strains and sex of animals that are assumed to } \\
\text { have different water consumption rates. }\end{array}$} \\
\hline
\end{tabular}




\section{Appendix T. Fluoride Administered and Converted Doses (Range across Studies for a Given ppm Level)}

\section{Table T-1. Summary of Fluoride Administered and Converted Doses (Across Studies)}

\begin{tabular}{|c|c|c|}
\hline $\begin{array}{c}\text { Fluoride } \\
\text { Concentrations }{ }^{1} \\
\text { ppm }\end{array}$ & $\begin{array}{c}\text { Fluoride } \\
\text { Concentrations }{ }^{1} \\
\text { mg/kg-d }\end{array}$ & References \\
\hline \multicolumn{3}{|c|}{ Oral Drinking Water } \\
\hline 0.20 & $0.03-0.04$ & Ekambaram and Paul $(2001 ; 2002)$ \\
\hline 0.23 & 0.03 & Gao et al. (2009a); Liu et al. (2010); Liu et al. (2009) \\
\hline 0.30 & 0.07 & Kivrak (2012) \\
\hline 0.34 & 0.05 & Jiang et al. (2014a) \\
\hline 0.45 & 0.12 & Zhang et al. (2001); Zhang et al. (1999) \\
\hline 0.50 & 0.07 & $\begin{array}{l}\text { Dong et al. (2015a); Dong et al. (2015b); Dong et al. (2015c); Gao et } \\
\text { al. (2008a); Jetti et al. (2016); Liu et al. (2011); Raghu et al. (2013) }\end{array}$ \\
\hline 0.54 & 0.08 & Bataineh and Nusier (2006) \\
\hline 0.60 & $0.01-0.13$ & Wang et al. (2004); Wu et al. (2008) \\
\hline 0.70 & $0.05-0.10$ & Chioca et al. (2008); Pereira et al. (2011) \\
\hline 0.90 & 0.23 & Liu et al. (2014) \\
\hline 1.0 & $0.14-0.21$ & Basha et al. (2011); Gao et al. (2008b); Wu et al. (2008) \\
\hline 1.1 & 0.18 & Zhang et al. (2009) \\
\hline 1.4 & 0.35 & Xu et al. (2001) \\
\hline 2.3 & $0.27-0.59$ & $\begin{array}{l}\text { Bartos et al. (2015); Gao et al. (2009a); Gao et al. (2008a); Gao et al. } \\
\text { (2008b); Liu et al. (2014); Liu et al. (2011); Liu et al. (2010); Liu et al. } \\
\text { (2009); Zhang et al. (2009); Zhang et al. (2001); Zhang et al. (1999); } \\
\text { Zhu et al. (2012) }\end{array}$ \\
\hline 2.7 & 0.7 & Xu et al. (2001) \\
\hline 4.5 & $0.54-1.19$ & $\begin{array}{l}\text { Bartos et al. (2015); Liu et al. (2014); Zhang et al. (2009); Zhang et al. } \\
\text { (2001); Zhang et al. (1999); Zhu et al. (2012) }\end{array}$ \\
\hline 5.0 & 1.1 & Wu et al. (2008) \\
\hline 5.4 & 1.4 & Xu et al. (2001) \\
\hline 9.1 & 1.34 & Zhu et al. (2012) \\
\hline 10.0 & 2.6 & Sun et al. (2008) \\
\hline 11.0 & 2.8 & Han et al. (2014) \\
\hline 11.3 & 1.6 & Jiang et al. (2014a) \\
\hline 11.8 & 1.8 & Jain et al. (2015) \\
\hline 13.6 & 2.2 & Liu (1989) \\
\hline 18.5 & 5.0 & Băran-Poesina et al. (2013) \\
\hline 22.0 & 5.6 & Han et al. (2014) \\
\hline
\end{tabular}


Systematic Literature Review on the Effects of Fluoride on Learning and Memory in Animal Studies

\begin{tabular}{|c|c|c|}
\hline $\begin{array}{c}\text { Fluoride } \\
\text { Concentrations }^{1} \\
\text { ppm }\end{array}$ & $\begin{array}{c}\text { Fluoride } \\
\text { Concentrations }{ }^{1} \\
\text { mg/kg-d }\end{array}$ & References \\
\hline 22.6 & $2.3-5.8$ & $\begin{array}{l}\text { Chen and Geng (2011); Chioca et al. (2008); Dong et al. (2015b); } \\
\text { Dong et al. (2015c); El-lethey et al. (2011); El-lethey et al. (2010); Gao } \\
\text { et al. (2009a); Gao et al. (2008a); Gao et al. (2008b); Jiang et al. } \\
\text { (2014a); Li et al. (2015); Liu et al. (2011); Liu et al. (2010); Liu et al. } \\
\text { (2009) Ma et al. (2015) }\end{array}$ \\
\hline 25.0 & 5.3 & Wu et al. (2008) \\
\hline 27.2 & 4.4 & Liu (1989) \\
\hline 33.2 & 3 & Whitford et al. (2009) \\
\hline 37.1 & 10 & Băran-Poesina et al. (2013) \\
\hline 40.0 & 9.6 & Kivrak (2012) \\
\hline 45.0 & 11.5 & Han et al. (2014) \\
\hline $45.2-45.3$ & $4.9-12.3$ & $\begin{array}{l}\text { Balaji et al. (2015); Chen and Geng (2011); Chioca et al. (2008); El- } \\
\text { lethey et al. (2011); El-lethey and Kamel (2011); El-lethey et al. } \\
\text { (2010); El-lethey and Shaheed (2011); Hong et al. (2005); Jiang et al. } \\
\text { (2014a); Wang et al. (2006); Wang et al. (2004); Wu et al. (2006) } \\
\text { Bataineh and Nusier (2006); Ma et al. (2015); Pereira et al. (2011) }\end{array}$ \\
\hline 50.0 & 13.1 & Sun et al. (2008) \\
\hline 54.3 & $7.5-14.7$ & Bhatnagar et al. (2002); Jiang et al. (2014b) \\
\hline 61.4 & 9.1 & Banji et al. (2013) \\
\hline 67.9 & $9.8-18.4$ & $\begin{array}{l}\text { Chen and Geng (2011); Niu et al. (2014); Niu et al. (2009); Niu et al. } \\
\text { (2008); Shen et al. (2004) }\end{array}$ \\
\hline 75.0 & 10.4 & Balayssac et al. (2002) \\
\hline 85.6 & 6 & Whitford et al. (2009) \\
\hline 90.5 & $13.8-24.5$ & Balaji et al. (2015); Wang et al. (2006) \\
\hline 100 & $14.8-26.3$ & $\begin{array}{l}\text { Basha et al. (2011); Jetti et al. (2016); Mullenix et al. (1995); Raghu et } \\
\text { al. (2013); Sun et al. (2008) (6-wk exposure) }\end{array}$ \\
\hline 125 & 19 & Mullenix et al. (1995) (16-wk exposure) \\
\hline 135.7 & 18.8 & Bataineh and Nusier (2006) \\
\hline 150 & 20.8 & Balayssac et al. (2002) \\
\hline 155.2 & 12 & Whitford et al. (2009) \\
\hline 200 & 32 & Basha et al. (2011) \\
\hline 226 & $36.3-110.7$ & Ekambaram and Paul (2001; 2002); Ekambaram and Paul (2003) \\
\hline 271.5 & 40.0 & Basha and Sujitha (2012) \\
\hline \multicolumn{3}{|l|}{ Oral Diet } \\
\hline 1.8 & 0.3 & Gui et al. (2010) \\
\hline 4.2 & 0.6 & Elliott (1967) \\
\hline 29.8 & 4.3 & Gui et al. (2010) \\
\hline 42.4 & 6.1 & Elliott (1967) \\
\hline
\end{tabular}


Systematic Literature Review on the Effects of Fluoride on Learning and Memory in Animal Studies

\begin{tabular}{ccl}
\hline $\begin{array}{c}\text { Fluoride } \\
\text { Concentrations } \\
\text { ppm }\end{array}$ & $\begin{array}{c}\text { Fluoride } \\
\text { Concentrations }{ }^{1} \\
\text { mg/kg-d }\end{array}$ & \\
\hline Gavage & & References \\
\hline 0.45 & 0.07 & Wei et al. (2014) \\
7.1 & 1.1 & Bera et al. (2007); Flace et al. (2010) \\
14.1 & 2.3 & Bera et al. (2007); Flace et al. (2010) \\
14.8 & 2.3 & Sarkozi et al. (2015) \\
22.6 & 3.4 & Wei et al. (2014) \\
31.3 & 4.5 & Gopal et al. (2006) \\
33.3 & 5.0 & Rumiantsev et al. (1988) \\
45.3 & 6.7 & Zhang et al. (2013) \\
56.5 & 9.1 & Paul et al. (1998) \\
62.6 & 9.1 & Gopal et al. (2006) \\
62.7 & 9.1 & Gao et al. (2009b) \\
112.9 & 18.1 & Paul et al. (1998) \\
\hline
\end{tabular}

Subcutaneous or Intraperitoneal Injection

- $\quad 0.06$ (9 prenatal Mullenix et al. (1995)

injections)

9.1 (single injection) Reddy and Karnati (2015)

In general, the administered chemical in studies is sodium fluoride $(\mathrm{NaF})$, and not all studies provide $\mathrm{F}$ equivalents. Dose levels are presented here as average daily doses of fluoride $(\mathrm{mg} / \mathrm{kg}-\mathrm{d})$ and equivalent water concentrations of fluoride (ppm). As needed, dose conversions were made using US EPA $(1988 ; 1994)$ default food or water consumption rates and body weights (using subchronic age and experiment duration) for the species/strain and sex of the animal of interest. Dose levels in $\mathrm{mg} / \mathrm{kg}-\mathrm{d}$ can vary for a given ppm across different studies if the studies use different species/strains and sex of animals that are assumed to have different water consumption rates. 


\section{Appendix U. Pharmacokinetics and Concentrations of Fluoride Measured in Tissue, Blood, Urine, or Bone}

The predominant route and source of human exposure to fluoride is by oral ingestion of drinking water (either with naturally occurring or added fluoride), food, and dental products containing fluorides (US DHHS 2015; US EPA 2010b). Fluoride from water and dental products is in the form of fluoride ion, released in solution from water-soluble fluorides such as sodium fluoride, hydrogen fluoride, fluorosilicic acid, or sodium monofluorophosphate (Ekstrand et al. 1978; Spak et al. 1982). Fluoride compounds with low water solubility such as calcium fluoride, magnesium fluoride, or aluminum fluoride are poorly absorbed (IPCS 2002). The pharmacokinetics of fluoride are driven primarily by $\mathrm{pH}$ and storage in bone (NRC 2006). The acidic environment of the stomach promotes the conversion of fluoride ion to hydrogen fluoride, which is rapidly absorbed by passive diffusion from the stomach and small intestine (Whitford and Pashley 1984). Approximately 70-90\% of ingested fluoride is absorbed in the alimentary tract and, for very soluble forms such as sodium fluoride, absorption is almost $100 \%$ (NRC 2006). Peak plasma concentrations are reached within $30 \mathrm{~min}$ (IPCS 2002). Calcified tissues such as bone and teeth readily incorporate fluoride (NRC 2006).

When taken with food, the rate of fluoride absorption from the gut and overall bioavailability are both lowered, thought due to binding of fluoride ions to calcium or other food constituents (Ekstrand and Ehrnebo 1979; Shulman and Vallejo 1990; Trautner and Einwag 1989). In healthy young adults, blood levels of fluoride, when expressed as micrograms per liter, approximate the consumed water concentration of fluoride expressed as mg/L (NRC 1993). Fluoride is cleared from plasma primarily through uptake by bone and excretion in urine, and clearance by these two routes is approximately equal in healthy adult humans (Whitford 1996). Clearance of fluoride from plasma occurs with a half-life estimated between 3 and 10 hours, depends on dose, and is affected by renal function and ongoing bone accretion and dissolution (Waterhouse et al. 1980). A significant amount of fluoride is cleared from blood by deposition into mineralized tissues. In animals and humans, approximately $99 \%$ of the body burden of fluoride is in bones and teeth (Hamilton 1992; Kaminsky et al. 1990). Fluoride is incorporated into the crystal lattice structure of mineralized tissues in the form of partially fluoridated hydroxyapatite (WHO 1994).

In infants and children, fluoride intake varies with age and diet, and relative clearance from plasma into mineralized tissues is extensive; clearance in children is larger compared to adults because of their growing skeletal system (Ekstrand et al. 1994; Ophaug et al. 1980). The features of developing versus mature bone provide more surface area for reaction with fluoride (Whitford 1996). In the elderly, blood fluoride levels can be elevated because of reduced capacity of bone to absorb fluoride, diminishing bone mass, and impaired renal function (IPCS 2002). Fluoride freely crosses the placenta, although concentrations in breast milk are lower than in plasma (Shen and Taves 1974). Whether consumption of fluoridated versus nonfluoridated water by the mother materially affects breast-milk fluoride concentrations is not yet settled (Dabeka et al. 1986; Ekstrand et al. 1981). Formula-fed infants are exposed to higher levels of fluoride than breast-fed infants, particularly when formula is reconstituted with fluoridated water (IPCS 2002). The blood-brain barrier is thought to reduce fluoride transfer (Whitford 1996).

Rats appear to require higher chronic exposure than humans to achieve the same plasma and bone fluoride concentrations, with one estimate of five times higher water concentrations 
required to achieve the same plasma concentration (Dunipace et al. 1995). With respect to bone, one study estimated that "humans incorporate fluoride $\sim 18$ times more readily than rats when the rats are on a normal calcium diet" (Turner et al. 1995). Several studies in rodents cited in this document have measured serum or plasma levels of fluoride following exposures to various elevated levels in drinking water (Table U-1). These studies have reported relationships between fluoride concentrations in drinking water and plasma/serum ranging from approximately 70 8000 times higher levels in water than were measured in plasma/serum (Table U-2). This variability is perhaps not surprising because the ratio for a substance that is relatively rapidly cleared from blood could be affected by many factors including (1) the time between removal from or the last exposure to drinking water, which is not reported in studies; (2) the age of the animal and the related capacity of its mineralized tissues to take up circulating fluoride; and (3) potential interstrain and species differences in renal clearance rates.

Table U-1. Concentrations of Fluoride Measured in Tissue, Blood, Urine, or Bone

\begin{tabular}{|c|c|c|c|c|c|c|}
\hline \multirow[b]{2}{*}{ Study } & \multirow[b]{2}{*}{ Treatment $^{1}$} & \multirow{2}{*}{$\begin{array}{c}\text { Fluoride } \\
\text { Concentration } \\
\text { ppm (mg/kg-d) }\end{array}$} & \multicolumn{4}{|c|}{ Internal Doses ${ }^{2}$} \\
\hline & & & $\begin{array}{c}\text { Brain or Other } \\
\text { Tissue }\end{array}$ & $\begin{array}{l}\text { Serum/ } \\
\text { Plasma }\end{array}$ & Urine & Bone \\
\hline $\begin{array}{l}\text { Balaji et al. } \\
\text { (2015) }\end{array}$ & $\begin{array}{l}\text { Swiss albino mouse } \\
\text { (adult, } 6 \text { q); drinking } \\
\text { water for } 30 \text { days }\end{array}$ & $\begin{array}{c}0(0), 45.25 \\
(12.27), 90.49 \\
\quad(24.53)\end{array}$ & $0.083,0.24,0.42$ & - & - & - \\
\hline \multirow[t]{2}{*}{$\begin{array}{l}\text { Balayssac et al. } \\
(2002)\end{array}$} & $\begin{array}{l}\text { Sprague Dawley rat } \\
\text { (adult, } 10 \widehat{\jmath} \text { ); drinking } \\
\text { water for } 104 \text { days }\end{array}$ & $\begin{array}{c}0(0), 75(10.393) \\
150(20.787)\end{array}$ & - & $1,5.5,10.9 \mu \mathrm{M}$ & - & - \\
\hline & $\begin{array}{l}\text { Lou/C rat (adult, } 12 \\
\text { đ); drinking water for } \\
195 \text { days }\end{array}$ & $\begin{array}{l}0(0), 150 \\
(20.787)\end{array}$ & & $1.1,12.3 \mu \mathrm{M}$ & & \\
\hline $\begin{array}{l}\text { Banji et al. } \\
\text { (2013) }\end{array}$ & $\begin{array}{l}\text { Wistar rat (adult P0 } \\
\text { generation, } 6 \text { q); } \\
\text { drinking water from } \\
\text { GD6-PND15 }\end{array}$ & $0(0), 61.37(9.05)$ & - & $\begin{array}{c}P 0 \\
0.12,0.32 \text { (day } \\
15 \text { ) } \\
0.14,0.42 \text { (day } \\
30 \text { ) }\end{array}$ & - & - \\
\hline \multirow[t]{3}{*}{$\begin{array}{l}\text { Basha et al. } \\
\text { (2011) }\end{array}$} & $\begin{array}{l}\text { Wistar rat (adult P0 } \\
\text { generation, } 8 \text { +); } \\
\text { drinking water from } \\
\text { GD0 through lactation }\end{array}$ & $\begin{array}{c}0(0), 100 \\
(16.025), 200 \\
(32.051)\end{array}$ & $\begin{array}{c}F 1 \\
0.318,1.83,2.32 \mathrm{CB} \\
0.672,2.28,3.43 \mathrm{CC} \\
0.258,0.993,1.93 \\
\mathrm{HC} \\
0.358,2.12,3.09 \mathrm{MO}\end{array}$ & - & - & - \\
\hline & & & $\begin{array}{c}F 2 \\
0.32,2.07,2.61 \mathrm{CB} \\
0.675,2.89,3.66 \mathrm{CC} \\
0.256,1.24,2.34 \mathrm{HC} \\
0.362,2.78,3.53 \mathrm{MO}\end{array}$ & & & \\
\hline & & & $\begin{array}{c}F 3 \\
0.319,2.51,2.87 \mathrm{CB} \\
0.673,3.11,3.92 \mathrm{CC} \\
0.26,1.65,2.73 \mathrm{HC} \\
0.359,3.03,3.84 \mathrm{MO}\end{array}$ & & & \\
\hline
\end{tabular}


Systematic Literature Review on the Effects of Fluoride on Learning and Memory in Animal Studies

\begin{tabular}{|c|c|c|c|c|c|c|}
\hline \multirow[b]{2}{*}{ Study } & \multirow[b]{2}{*}{ Treatment $^{1}$} & \multirow{2}{*}{$\begin{array}{c}\text { Fluoride } \\
\text { Concentration } \\
\text { ppm (mg/kg-d) }\end{array}$} & \multicolumn{4}{|c|}{ Internal Doses ${ }^{2}$} \\
\hline & & & $\begin{array}{c}\text { Brain or Other } \\
\text { Tissue }\end{array}$ & $\begin{array}{l}\text { Serum/ } \\
\text { Plasma }\end{array}$ & Urine & Bone \\
\hline $\begin{array}{l}\text { Dong et al. } \\
(2015 \mathrm{c})\end{array}$ & $\begin{array}{l}\text { Sprague Dawley rat } \\
\text { (adult P0 generation, } \\
30 \lesssim+\text { ); drinking } \\
\text { water from GD0- } \\
\text { PND28 }\end{array}$ & $\begin{array}{c}0.23(0.03), 22.6 \\
(3.27)\end{array}$ & - & - & $\begin{array}{c}P O \\
1.7,2.16\end{array}$ & $\begin{array}{c}P 0 \\
34.67 \\
211.07\end{array}$ \\
\hline $\begin{array}{l}\text { Dong et al. } \\
(2015 b)\end{array}$ & $\begin{array}{l}\text { Sprague Dawley rat } \\
\text { (adult, } 30 \lesssim+\text { ); } \\
\text { drinking water for } 10 \\
\text { months }\end{array}$ & $\begin{array}{c}0.23(0.03), 22.6 \\
(3.27)\end{array}$ & - & - & $1.7,2.16$ & $1.52,2.31$ \\
\hline $\begin{array}{l}\text { Ekambaram and } \\
\text { Paul (2001) V } \\
2001\end{array}$ & $\begin{array}{l}\text { Wistar rat (adult, } 8 \\
\text { ㅇ); drinking water for } \\
60 \text { days }\end{array}$ & $\begin{array}{c}0(0), 226.24 \\
\quad(36.26)\end{array}$ & - & $0.22,1.94$ & - & - \\
\hline $\begin{array}{l}\text { Ekambaram and } \\
\text { Paul (2002) }\end{array}$ & $\begin{array}{l}\text { Wistar rat (adult, } 8 \\
\text { )ㅏㅁ drinking water for } \\
60 \text { days }\end{array}$ & $\begin{array}{c}0.2(0.04), 226 \\
(40.4)\end{array}$ & - & $0.22,1.94$ & - & - \\
\hline $\begin{array}{l}\text { Gao et al. } \\
(2009 a)\end{array}$ & $\begin{array}{l}\text { Sprague Dawley rat } \\
\text { (adult, } 8 \partial^{\lambda}+\text { ); } \\
\text { drinking water for } 6 \\
\text { months }\end{array}$ & $\begin{array}{c}0.23(0.03), 2.26 \\
(0.33), 22.6(3.27)\end{array}$ & - & - & $\begin{array}{l}1.02 \\
2.59 \\
5.96\end{array}$ & $\begin{array}{l}669.4 \\
1135.2 \\
1304.3\end{array}$ \\
\hline $\begin{array}{l}\text { Gao et al. } \\
(2009 b)\end{array}$ & $\begin{array}{l}\text { Sprague Dawley rat } \\
\text { (adult, } 8 \delta^{\lambda}+\text { ); } \\
\text { gavage for } 3 \text { months }\end{array}$ & $0(0), 62.68(9.05)$ & - & $0.018,7.135$ & $\begin{array}{l}1.462 \\
64.966\end{array}$ & \\
\hline Gui et al. (2010) & $\begin{array}{l}\text { Sprague Dawley rat } \\
\text { (adult P0 generation, } \\
9 \delta^{\lambda}+\text { ); diet for } 6 \\
\text { months prior to } \\
\text { mating, through } \\
\text { lactation F1 (10 } \delta^{\wedge}+\text { ); } \\
\text { diet through PND30 }\end{array}$ & $\begin{array}{c}F 1 \\
1.77(0.26), 29.81 \\
(4.3)\end{array}$ & $\begin{array}{c}F 1 \\
0.36,0.74\end{array}$ & - & $\begin{array}{c}F 1 \\
0.98 \\
8.52\end{array}$ & $\begin{array}{c}F 1 \\
1124,1873\end{array}$ \\
\hline $\begin{array}{l}\text { Han et al. } \\
(2014)\end{array}$ & $\begin{array}{l}\text { Kunming mouse } \\
\text { (adult, } 15 \lesssim \text { ); drinking } \\
\text { water for } 6 \text { months }\end{array}$ & $\begin{array}{l}0(0), 11(2.82) 22 \\
(5.63), 45(11.52)\end{array}$ & $\begin{array}{c}23.5,34.2,38.8,39.6 \\
\text { HC }\end{array}$ & - & - & - \\
\hline $\begin{array}{l}\text { Jiang et al. } \\
(2014 b)\end{array}$ & $\begin{array}{l}\text { Sprague Dawley rat } \\
\text { (adult, } 8 \gtrsim \text { ); drinking } \\
\text { water for } 3 \text { months }\end{array}$ & $0(0), 54.3(7.52)$ & $\begin{array}{c}0.25,1.19 \text { cortex } \\
0.24,1.14 \mathrm{HC}\end{array}$ & $0.09,0.4$ & - & - \\
\hline Li et al. (2015) & $\begin{array}{l}\text { Wistar rat (juvenile- } \\
\text { adult, } 10 \delta^{\lambda} \text { ); drinking } \\
\text { water for } 3 \text { months }\end{array}$ & $0(0), 22.6(3.34)$ & - & - & $\begin{array}{l}6.67 \\
22.5\end{array}$ & $\begin{array}{c}0.0646 \\
0.189\end{array}$ \\
\hline $\begin{array}{l}\text { Mullenix et al. } \\
\text { (1995) }\end{array}$ & $\begin{array}{l}\text { Sprague Dawley rat } \\
\text { (adult, } 20 \text { + ); drinking } \\
\text { water for } 6 \text { weeks }\end{array}$ & $0(0), 100(21.43)$ & $\begin{array}{c}0.406,0.252 \mathrm{BG} \\
0.358,0.325 \mathrm{CB} \\
0.479,0.602 \text { cortex } \\
0.258,0.79 \mathrm{HC} \\
0.396,0.308 \mathrm{HT} \\
0.634,0.306 \mathrm{MB} \\
0.609,1.28 \mathrm{MO}\end{array}$ & $0.01,0.077$ & - & - \\
\hline Niu et al. (2009) & $\begin{array}{l}\text { Wistar albino rat } \\
\text { (adult P0 generation, } \\
\text { o); drinking water } \\
\text { from GD0 to PND21 } \\
\text { F1 (8); } 12 \text { weeks after } \\
\text { lactation }\end{array}$ & $\begin{array}{c}F 1 \\
0(0), 67.87 \\
(10.88)\end{array}$ & - & $\begin{array}{c}F 1 \\
0.59,2.78\end{array}$ & - & - \\
\hline
\end{tabular}


Systematic Literature Review on the Effects of Fluoride on Learning and Memory in Animal Studies

\begin{tabular}{|c|c|c|c|c|c|c|}
\hline \multirow[b]{2}{*}{ Study } & \multirow[b]{2}{*}{ Treatment $^{1}$} & \multirow{2}{*}{$\begin{array}{c}\text { Fluoride } \\
\text { Concentration } \\
\text { ppm (mg/kg-d) }\end{array}$} & \multicolumn{4}{|c|}{ Internal Doses ${ }^{2}$} \\
\hline & & & $\begin{array}{c}\text { Brain or Other } \\
\text { Tissue }\end{array}$ & $\begin{array}{l}\text { Serum/ } \\
\text { Plasma }\end{array}$ & Urine & Bone \\
\hline $\begin{array}{l}\text { Reddy and } \\
\text { Karnati (2015) }\end{array}$ & $\begin{array}{l}\text { Wistar rat (adult, } 6 \\
\nwarrow) \text {; intraperitoneal } \\
\text { injection for } 14 \text { days }\end{array}$ & $\begin{array}{l}0,9.05 \mathrm{mg} / \mathrm{kg} \text { bw } \\
\text { (single injection) }\end{array}$ & $\begin{array}{c}0.43,0.77 \\
\text { gastrocnemius muscle } \\
\text { tissue }\end{array}$ & - & - & - \\
\hline $\begin{array}{l}\text { Rumiantsev et } \\
\text { al. (1988) }\end{array}$ & $\begin{array}{l}\text { Rat (adult); gavage for } \\
30 \text { days }\end{array}$ & $0(0), 33.32(5)$ & - & - & $\begin{array}{l}14,744 \\
\mu \mathrm{g} / 24 \mathrm{hr} \\
\text { urine }\end{array}$ & $\begin{array}{l}2.3, \\
10.6 \mathrm{mg} \% \\
\text { thigh bone }\end{array}$ \\
\hline $\begin{array}{l}\text { Whitford et al. } \\
\text { (2009) }\end{array}$ & $\begin{array}{l}\text { Sprague Dawley rat } \\
\text { (adult, } 8 \text { +); drinking } \\
\text { water for } 8 \text { months }\end{array}$ & $\begin{array}{c}0(0), 33.2(3) \\
85.6(6), 155.2 \\
(12)\end{array}$ & $\begin{array}{c}0.082,3.9,6.42,18.1 \\
\mu \mathrm{mol} / \mathrm{kg} \mathrm{ww}\end{array}$ & $\begin{array}{c}0.32,15.27 \\
25.72,97.56 \\
\mu \mathrm{mol} / \mathrm{L}\end{array}$ & - & $\begin{array}{c}\text { 150, 5408, } \\
9497, \\
\text { 14,847 ash } \\
\text { femur distal } \\
\text { epiphysis }\end{array}$ \\
\hline
\end{tabular}


Table U-2. Comparison of Drinking Water Concentration and Levels of Fluoride in Serum or Plasma

\begin{tabular}{|c|c|c|c|c|c|}
\hline Study & Treatment & $\begin{array}{c}\text { Fluoride } \\
\text { Concentration } \\
\text { ppm } \\
(\mathbf{m g} / \mathbf{k g}-\mathbf{d})\end{array}$ & $\begin{array}{l}\text { Internal } \\
\text { Doses } \\
\text { (Serum or } \\
\text { Plasma) }\end{array}$ & $\begin{array}{l}\text { Risk } \\
\text { of } \\
\text { Bias }\end{array}$ & Reporting Quality of LOD, Analytical Methods, and Timing of Sample Collection \\
\hline \multirow[t]{6}{*}{$\begin{array}{l}\text { Balayssac et } \\
\text { al. }(2002)\end{array}$} & $\begin{array}{l}\text { Sprague Dawley } \\
\text { rat (adult, } 10 \hat{\jmath}) \text {; } \\
\text { drinking water } \\
\text { for } 104 \text { days }\end{array}$ & $\begin{array}{l}0(0), 75 \\
(10.393), 150 \\
(20.787)\end{array}$ & $\begin{array}{l}1,5.5,10.9 \mu \mathrm{M} \\
\text { (equivalent to } \\
0.019,0.1045 \\
0.2071 \mathrm{mg} / \mathrm{L} \text { ) }\end{array}$ & ++ & LOD: The method was linear from 0.01 to $100 \mathrm{mg} / \mathrm{L}$ fluoride with all values within this range. \\
\hline & & & & ++ & $\begin{array}{l}\text { Analytical method: Samples were collected after slight anesthesia. A combined fluoride- } \\
\text { selective electrode ISEC 301F linked to a Meterlab PHM250® ion analyzer was used to measure } \\
\text { plasma F concentrations. }\end{array}$ \\
\hline & & & & - & $\begin{array}{l}\text { Timing: Plasma F levels were measured at the end of the experiment, but when the experiment } \\
\text { ended was not clear, as at least one outcome appears to have been measured } 8 \text { days after } \\
\text { exposure ended (based on Figure 1). }\end{array}$ \\
\hline & $\begin{array}{l}\text { Lou/C rat (adult, } \\
12 \overbrace{}^{\lambda}) \text {; drinking } \\
\text { water for } 195 \\
\text { days }\end{array}$ & $\begin{array}{l}0(0), 150 \\
(20.787)\end{array}$ & $\begin{array}{l}1.1,12.3 \mu \mathrm{M} \\
\text { (equivalent to } \\
0.0209, \\
0.2337 \mathrm{mg} / \mathrm{L} \text { ) }\end{array}$ & ++ & LOD: The method was linear from 0.01 to $100 \mathrm{mg} / \mathrm{L}$ fluoride with all values within this range. \\
\hline & & & & ++ & $\begin{array}{l}\text { Analytical method: Samples were collected after slight anesthesia. A combined fluoride- } \\
\text { selective electrode ISEC 301F linked to a Meterlab PHM250® ion analyzer was used to measure } \\
\text { plasma F concentration. }\end{array}$ \\
\hline & & & & + & $\begin{array}{l}\text { Timing: Plasma F levels were measured at the end of the experiment, which appears to be at the } \\
\text { end of treatment based on the time that the outcome was measured throughout treatment. }\end{array}$ \\
\hline \multirow[t]{3}{*}{$\begin{array}{l}\text { Banji et al. } \\
(2013)\end{array}$} & $\begin{array}{l}\text { Wistar rat (adult } \\
\text { P0 generation, } 6 \\
\text { o); drinking } \\
\text { water from } \\
\text { GD6-PND15 }\end{array}$ & $\begin{array}{l}0(0), 61.37 \\
(9.05)\end{array}$ & $\begin{array}{l}P 0 \\
0.12,0.32 \text { (day } \\
15) \mathrm{mg} / \mathrm{L} 0.14 \text {, } \\
0.42(\text { day } \\
30) \mathrm{mg} / \mathrm{L}\end{array}$ & - & LOD: No LOD was provided. \\
\hline & & & & + & $\begin{array}{l}\text { Analytical method: Fluoride levels were determined using a potentiometer with an ion selective } \\
\text { electrode. No other specifics were provided. }\end{array}$ \\
\hline & & & & + & $\begin{array}{l}\text { Timing: Serum fluoride levels were determined by collecting } 0.5 \mathrm{~mL} \text { blood from the tail vein of } \\
\text { the dam on GD20 (corresponding to } 15 \text { th day of treatment) and PND11 (corresponding with } 30 \text { th } \\
\text { day of treatment), but was not measured at termination of treatment (PND15) or in the pups at } \\
\text { the end of treatment. }\end{array}$ \\
\hline
\end{tabular}




\begin{tabular}{|c|c|c|c|c|c|}
\hline Study & Treatment & $\begin{array}{c}\text { Fluoride } \\
\text { Concentration } \\
\text { ppm } \\
(\mathbf{m g} / \mathbf{k g - d})\end{array}$ & $\begin{array}{l}\text { Internal } \\
\text { Doses } \\
\text { (Serum or } \\
\text { Plasma) } \\
\end{array}$ & $\begin{array}{l}\text { Risk } \\
\text { of } \\
\text { Bias }\end{array}$ & Reporting Quality of LOD, Analytical Methods, and Timing of Sample Collection \\
\hline \multirow[t]{3}{*}{$\begin{array}{l}\text { Ekambaram } \\
\text { and Paul } \\
(2001)\end{array}$} & $\begin{array}{l}\text { Wistar rat (adult, } \\
8 \text { P); drinking } \\
\text { water for } 60 \\
\text { days }\end{array}$ & $\begin{array}{l}0(0), 226.24 \\
(36.26)\end{array}$ & $\begin{array}{l}0.22 \\
1.94 \mathrm{mg} / \mathrm{L}\end{array}$ & - & LOD: No LOD was provided. \\
\hline & & & & ++ & $\begin{array}{l}\text { Analytical method: Serum fluoride was determined by the method using a fluoride ion specific } \\
\text { electrode and a Fisher "accumet" model } 425 \mathrm{pH} / \mathrm{mV} \text { digital meter. One } \mathrm{mL} \text { of serum was mixed } \\
\text { with } 10 \mathrm{~mL} \text { of total ionic strength adjusting buffer in a small plastic beaker. The solution was } \\
\text { mixed thoroughly by using a magnetic stirrer. Then the electrode was immersed in the sample } \\
\text { solution. After the reading stabilized, the millivolt reading was recorded and expressed in } \mathrm{mg} / \mathrm{L} \text {. }\end{array}$ \\
\hline & & & & - & $\begin{array}{l}\text { Timing: When the blood was collected for } \mathrm{F} \text { analysis was not stated. Blood was stated to be } \\
\text { collected only at sacrifice for acetylcholinesterase measurement, but how long it was between } \\
\text { treatment and sacrifice was not reported. }\end{array}$ \\
\hline \multirow[t]{3}{*}{$\begin{array}{l}\text { Ekambaram } \\
\text { and Paul } \\
(2002)\end{array}$} & $\begin{array}{l}\text { Wistar rat (adult, } \\
8 \text { + }) \text {; drinking } \\
\text { water for } 60 \\
\text { days }\end{array}$ & $\begin{array}{l}0.2(0.04), 226 \\
(40.4)\end{array}$ & $\begin{array}{l}0.22 \\
1.94 \mathrm{mg} / \mathrm{L}\end{array}$ & - & LOD: No LOD was provided. \\
\hline & & & & ++ & $\begin{array}{l}\text { Analytical method: Serum fluoride was determined by the method using a fluoride ion specific } \\
\text { electrode and a Fisher "accumet" model } 425 \mathrm{pH} / \mathrm{mV} \text { digital meter. One } \mathrm{mL} \text { of serum was mixed } \\
\text { with } 10 \mathrm{~mL} \text { of total ionic strength adjusting buffer in a small plastic beaker. The solution was } \\
\text { mixed thoroughly by using a magnetic stirrer. Then the electrode was immersed in the sample } \\
\text { solution. After the reading stabilized, the millivolt reading was recorded and expressed in } \mathrm{mg} / \mathrm{L} \text {. }\end{array}$ \\
\hline & & & & - & $\begin{array}{l}\text { Timing: Blood was stated to be collected at sacrifice, but how long after treatment the animals } \\
\text { were sacrificed was not noted. }\end{array}$ \\
\hline \multirow[t]{3}{*}{$\begin{array}{l}\text { Gao et al. } \\
(2009 a)\end{array}$} & $\begin{array}{l}\text { Sprague Dawley } \\
\text { rat (adult, } 8 \\
\supset^{1} \text { ); } \text {; gavage for } \\
3 \text { months }\end{array}$ & $\begin{array}{l}0(0), 62.68 \\
(9.05)\end{array}$ & $\begin{array}{l}0.018,7.135 \\
\mu \mathrm{g} / \mathrm{mL} \\
\text { (i.e., } \mathrm{mg} / \mathrm{L})\end{array}$ & + & $\begin{array}{l}\text { LOD: No LOD was provided in the publication, but the study cites another publication for the } \\
\text { methods used, which reported an LOD of } 0.012 \mu \mathrm{g} / \mathrm{mL} \text { (number from "Determination for } \\
\text { fluoride in serum-ion selective electrode method (WS / T 212-2001)") }\end{array}$ \\
\hline & & & & + & $\begin{array}{l}\text { Analytical method: Rats were decapitated, and blood samples were taken and stored in a } \\
\text { polyethylene tube with plug. Blood samples were centrifuged immediately to extract blood } \\
\text { serum samples, which were stored in a refrigerator at }-35^{\circ} \mathrm{C} \text {. All serum fluoride concentrations } \\
\text { were determined using method "Determination for fluoride in serum-ion selective electrode } \\
\text { method (WS / T 212-2001)" for two weeks to complete the measurement. }\end{array}$ \\
\hline & & & & - & $\begin{array}{l}\text { Timing: Blood was stated to be collected at sacrifice, but how long after treatment the animals } \\
\text { were sacrificed was not noted. Authors noted that animals were sacrificed after the learning tests } \\
\text { were complete, but did not indicate how many days after treatment this was. }\end{array}$ \\
\hline
\end{tabular}


Systematic Literature Review on the Effects of Fluoride on Learning and Memory in Animal Studies

\begin{tabular}{|c|c|c|c|c|c|}
\hline Study & Treatment & $\begin{array}{c}\text { Fluoride } \\
\text { Concentration } \\
\text { ppm } \\
(\mathbf{m g} / \mathbf{k g}-\mathbf{d})\end{array}$ & $\begin{array}{l}\text { Internal } \\
\text { Doses } \\
\text { (Serum or } \\
\text { Plasma) }\end{array}$ & $\begin{array}{l}\text { Risk } \\
\text { of } \\
\text { Bias }\end{array}$ & Reporting Quality of LOD, Analytical Methods, and Timing of Sample Collection \\
\hline \multirow[t]{3}{*}{$\begin{array}{l}\text { Mullenix et al. } \\
\text { (1995) }\end{array}$} & $\begin{array}{l}\text { Sprague Dawley } \\
\text { rat (adult, } 20 \text { \%); } \\
\text { drinking water } \\
\text { for } 6 \text { weeks }\end{array}$ & $\begin{array}{l}0(0), 100 \\
(21.43)\end{array}$ & $\begin{array}{l}0.01, \\
0.077 \mathrm{mg} / \mathrm{L}\end{array}$ & - & LOD: No LODs were provided. \\
\hline & & & & + & $\begin{array}{l}\text { Analytical method: All plasma fluoride concentrations were determined using an ion-specific } \\
\text { electrode, following hexamethyldisiloxane diffusion method. }\end{array}$ \\
\hline & & & & - & Timing: For the adult studies, when the blood samples were obtained was not reported. \\
\hline \multirow[t]{3}{*}{$\begin{array}{l}\text { Mullenix et al. } \\
\text { (1995) }\end{array}$} & $\begin{array}{l}\text { Sprague Dawley } \\
\text { rat (weanling, 6- } \\
19 \text { đ̇o); } \\
\text { drinking water } \\
\text { for } 6 \text { or } 20 \\
\text { weeks }\end{array}$ & $\begin{array}{l}0,75,100,125, \\
175\end{array}$ & & - & LOD: No LODs were provided. \\
\hline & & & & + & $\begin{array}{l}\text { Analytical method: All plasma fluoride concentrations were determined using an ion-specific } \\
\text { electrode, following hexamethyldisiloxane diffusion method. }\end{array}$ \\
\hline & & & & - & Timing: Fluoride levels were measured 3 weeks after exposure. \\
\hline \multirow[t]{3}{*}{$\begin{array}{l}\text { Niu et al. } \\
(2009)\end{array}$} & $\begin{array}{l}\text { Wistar albino rat } \\
\text { (adult P0 } \\
\text { generation, o }) \text {; } \\
\text { drinking water } \\
\text { from GD0 to } \\
\text { PND21 F1 (8); } \\
12 \text { weeks after } \\
\text { lactation }\end{array}$ & $\begin{array}{l}F 1 \\
0(0), 67.87 \\
(10.88)\end{array}$ & $\begin{array}{l}F 1 \\
0.59, \\
2.78 \mathrm{mg} / \mathrm{L} \\
\text { **units } \\
\text { unclear, listed } \\
\text { as } \mathrm{mg} / \mathrm{L}, \\
\mu \mathrm{g} / \mathrm{dL} \text {, and } \\
\mu \mathrm{g} / \mathrm{dL} \text { in article }\end{array}$ & - & LOD: No LODs were provided. \\
\hline & & & & - & Analytical method: Analytical methods were not reported. \\
\hline & & & & - & $\begin{array}{l}\text { Timing: How or when blood samples were collected for analysis was not reported. Animals } \\
\text { were sacrificed after the learning tests were complete, but authors gave no indication of how } \\
\text { many days after treatment this was. }\end{array}$ \\
\hline
\end{tabular}


Systematic Literature Review on the Effects of Fluoride on Learning and Memory in Animal Studies

\begin{tabular}{|c|c|c|c|c|c|}
\hline Study & Treatment & $\begin{array}{l}\text { Fluoride } \\
\text { Concentration } \\
\text { ppm } \\
(\mathbf{m g} / \mathrm{kg}-\mathrm{d})\end{array}$ & $\begin{array}{l}\text { Internal } \\
\text { Doses } \\
\text { (Serum or } \\
\text { Plasma) }\end{array}$ & $\begin{array}{c}\text { Risk } \\
\text { of } \\
\text { Bias }\end{array}$ & Reporting Quality of LOD, Analytical Methods, and Timing of Sample Collection \\
\hline \multirow[t]{3}{*}{$\begin{array}{l}\text { Whitford et al. } \\
(2009)\end{array}$} & $\begin{array}{l}\text { Sprague Dawley } \\
\text { rat (adult, } 8 \text { +); } \\
\text { drinking water } \\
\text { for } 8 \text { months }\end{array}$ & $\begin{array}{l}0(0), 33.2(3), \\
85.6(6), 155.2 \\
(12)\end{array}$ & $\begin{array}{l}0.32,15.27, \\
25.72,97.56 \\
\mu \mathrm{mol} / \mathrm{L} \\
\text { (equivalent to } \\
0.006,0.290, \\
0.489, \\
1.854 \mathrm{mg} / \mathrm{L} \text { ) }\end{array}$ & - & LOD: No LODs were provided. \\
\hline & & & & ++ & $\begin{array}{l}\text { Analytical method: Plasma was analyzed for fluoride using an ion-specific electrode (Orion } \\
\text { Research, Model 9409) and a reference electrode (Orion, Model 90-01) coupled to a } \\
\text { potentiometer (Orion Research, Model 720A) after overnight hexamethyldisiloxane (HMDS)- } \\
\text { facilitated diffusion. The next day the alkaline trap, } 50 \mu \mathrm{L} \text { of } 0.05 \mathrm{~N} \mathrm{NaOH} \text {, was allowed to air } \\
\text { dry. The NaOH crystals were dissolved by adding } 20 \mu \mathrm{L} \text { of } 0.20 \mathrm{~N} \text { acetic acid to form an acetate } \\
\text { buffer system (pH 5.2). This solution was transferred to the sensing surface of the inverted } \\
\text { reference electrode and the fluoride electrode was lowered until it contacted the solution. The } \\
\mathrm{mV} \text { potential was recorded after a stable reading had been obtained, which occurred within } 2-4 \\
\text { min. Three fluoride standards, each in triplicate, calculated to bracket the expected amounts of } \\
\text { fluoride in the different tissues, were diffused along with the samples. The r2 values for every } \\
\text { standard curve exceeded 0.97. Non-diffused standards also were analyzed. These standards were } \\
\text { prepared with the same reagents used to prepare the diffused standards and to have the same } \\
\text { concentrations as the diffused standards. Comparison of the mV potentials of the diffused and } \\
\text { non-diffused standards confirmed fluoride in the diffused standards had been completely trapped } \\
\text { and analyzed (range of agreement } 96-102 \% \text { ). }\end{array}$ \\
\hline & & & & - & $\begin{array}{l}\text { Timing: Authors noted that blood was collected } 4 \text { days after completion of the behavioral } \\
\text { studies, but when this was in terms of completion of the fluoride treatment was not clear } \\
\text { (presumably } 4 \text { days or more after completion of treatment). In addition, authors noted that } \\
\text { samples were placed in the refrigerator until analysis, but did not note the length of time until the } \\
\text { samples were analyzed. }\end{array}$ \\
\hline
\end{tabular}




\section{Appendix V. Background Fluoride Levels in Drinking Water and Diet}

Table V-1. Background Fluoride Levels in Drinking Water and Diet

\begin{tabular}{|c|c|c|c|c|}
\hline Reference & $\begin{array}{c}\text { Route of } \\
\text { Administration }\end{array}$ & Vehicle Control & $\begin{array}{l}\text { Background } \\
\text { Fluoride in } \\
\text { Drinking Water }\end{array}$ & $\begin{array}{l}\text { Background } \\
\text { Fluoride in Feed }\end{array}$ \\
\hline Balaji et al. (2015) & Drinking water & Nonfluoridated water & NR & NR \\
\hline Balayssac et al. (2002) & Drinking water & Deionized water & NR & $14 \mathrm{ppm}$ \\
\hline Banji et al. (2013) & Drinking water & Deionized water & NR & NR \\
\hline $\begin{array}{l}\text { Băran-Poesina et al. } \\
\text { (2013) }\end{array}$ & Drinking water & Distilled water & NR & NR \\
\hline Bartos et al. (2015) & Drinking water & Drinking water & NR & NR \\
\hline Basha et al. (2011) & Drinking water & Tap water $(<1$ ppm F) & $1 \mathrm{ppm}$ & NR \\
\hline $\begin{array}{l}\text { Basha and Sujitha } \\
\text { (2012) }\end{array}$ & Drinking water & Tap water & NR & NR \\
\hline $\begin{array}{l}\text { Bataineh and Nusier } \\
\text { (2006) }\end{array}$ & Drinking water & $\begin{array}{l}\text { Tap water } \\
(\mathrm{NaF}=1.2 \mathrm{ppm})\end{array}$ & $0.54 \mathrm{ppm}$ & NR \\
\hline Bera et al. (2007) & Gavage & Deionized water & NR & NR \\
\hline Bhatnagar et al. (2002) & Drinking water & $\begin{array}{l}\text { Deionized, defluoridated } \\
\text { water }\end{array}$ & NR & NR \\
\hline Chen and Geng (2011) & Drinking water & Distilled water & NR & NR \\
\hline Chioca et al. (2008) & Drinking water & $\begin{array}{l}\text { Drinking water } \\
(\mathrm{NaF}=1.54 \mathrm{ppm})\end{array}$ & $0.697 \mathrm{ppm}$ & NR \\
\hline Dong et al. (2015c) & Drinking water & Drinking water $(<0.5 \mathrm{ppm} F)$ & $<0.5 \mathrm{ppm}$ & $6.2 \mathrm{mg} / \mathrm{kg}$ \\
\hline Dong et al. (2015b) & Drinking water & Drinking water $(<0.5$ ppm F) & $<0.5 \mathrm{ppm}$ & $6.2 \mathrm{mg} / \mathrm{kg}$ \\
\hline Dong et al. (2015a) & Drinking water & Drinking water $(<0.5 \mathrm{ppm} F)$ & $<0.5 \mathrm{ppm}$ & $6.2 \mathrm{mg} / \mathrm{kg}$ \\
\hline $\begin{array}{l}\text { Ekambaram and Paul } \\
\text { (2001) }\end{array}$ & Drinking water & Drinking water $(0.2 \mathrm{ppm} \mathrm{F})$ & $0.2 \mathrm{ppm}$ & NR \\
\hline $\begin{array}{l}\text { Ekambaram and Paul } \\
(2002)\end{array}$ & Drinking water & Drinking water $(0.2 \mathrm{ppm} \mathrm{F})$ & $0.2 \mathrm{ppm}$ & NR \\
\hline $\begin{array}{l}\text { Ekambaram and Paul } \\
\text { (2003) }\end{array}$ & Drinking water & Tap water & NR & NR \\
\hline El-lethey et al. (2010) & Drinking water & Distilled water & NR & NR \\
\hline El-lethey et al. (2011) & Drinking water & Distilled water & NR & NR \\
\hline $\begin{array}{l}\text { El-lethey and Shaheed } \\
\text { (2011) }\end{array}$ & Drinking water & Distilled water & NR & NR \\
\hline $\begin{array}{l}\text { El-lethey and Kamel } \\
\text { (2011) }\end{array}$ & Drinking water & Distilled water & NR & NR \\
\hline Elliott (1967) & Dietary & $\begin{array}{l}\text { Well below } 1 \mathrm{ppm} \text { in } \\
\text { drinking water }\end{array}$ & $<1 \mathrm{ppm}$ & $\begin{array}{c}\text { Fluorine content } \\
0.002 \% \text { in feed }\end{array}$ \\
\hline
\end{tabular}


Systematic Literature Review on the Effects of Fluoride on Learning and Memory in Animal Studies

\begin{tabular}{|c|c|c|c|c|}
\hline Reference & $\begin{array}{c}\text { Route of } \\
\text { Administration }\end{array}$ & Vehicle Control & $\begin{array}{c}\text { Background } \\
\text { Fluoride in } \\
\text { Drinking Water }\end{array}$ & $\begin{array}{l}\text { Background } \\
\text { Fluoride in Feed }\end{array}$ \\
\hline Flace et al. (2010) & Gavage & Deionized water & NR & NR \\
\hline Gao et al. (2008a) & Drinking water & Drinking water $(<0.5 \mathrm{ppm} \mathrm{F})$ & $<0.5 \mathrm{ppm}$ & NR \\
\hline Gao et al. (2008b) & Drinking water & Drinking water $(<1 \mathrm{ppm} \mathrm{F})$ & $<1.0 \mathrm{ppm}$ & NR \\
\hline Gao et al. (2009a) & Drinking water & $\begin{array}{l}\text { Drinking water }(<0.5 \mathrm{ppm} \\
\mathrm{NaF})\end{array}$ & $<0.23 \mathrm{ppm}$ & NR \\
\hline Gao et al. (2009b) & Gavage & Deionized water & NR & NR \\
\hline Gopal et al. (2006) & Gavage & NR & NR & NR \\
\hline Gui et al. (2010) & Dietary & Tap water $(0.5$ ppm F) & $0.5 \mathrm{ppm}$ & $6.2 \mathrm{mg} / \mathrm{kg}$ \\
\hline Han et al. (2014) & Drinking water & Distilled water & NR & NR \\
\hline Hong et al. (2005) & Drinking water & Deionized water & NR & $25.57 \mathrm{mg} / \mathrm{kg}$ \\
\hline Jain et al. (2015) & Drinking water & NR & NR & NR \\
\hline Jetti et al. (2016) & Drinking water & Tap water $(0.5$ ppm F) & $0.5 \mathrm{ppm}$ & NR \\
\hline Jiang et al. (2014a) & Drinking water & Tap water $(0.34 \mathrm{mg} / \mathrm{L} \mathrm{F})$ & $0.34 \mathrm{ppm}$ & NR \\
\hline Jiang et al. (2014b) & Drinking water & Tap water & NR & NR \\
\hline Kivrak (2012) & Drinking water & $\begin{array}{l}\text { Drinking water } \\
(0.3 \mathrm{ppm} \mathrm{F})\end{array}$ & $0.3 \mathrm{ppm}$ & NR \\
\hline Li et al. (2015) & Drinking water & Distilled water & NR & NR \\
\hline Liu (1989) & Drinking water & Distilled water & NR & $2.9 \mathrm{ppm}$ \\
\hline Liu et al. (2009) & Drinking water & $\begin{array}{l}\text { Tap water } \\
(<0.5 \mathrm{ppm} \mathrm{NaF})\end{array}$ & $0.23 \mathrm{ppm}$ & NR \\
\hline Liu et al. (2010) & Drinking water & $\begin{array}{l}\text { Drinking water }(<0.5 \mathrm{ppm} \\
\mathrm{NaF})\end{array}$ & $0.23 \mathrm{ppm}$ & NR \\
\hline Liu et al. (2011) & Drinking water & Tap water $(<0.5$ ppm F $)$ & $0.5 \mathrm{ppm}$ & NR \\
\hline Liu et al. (2014) & Drinking water & Distilled water & NR & NR \\
\hline Ma et al. (2015) & Drinking water & Distilled water & NR & NR \\
\hline Mullenix et al. (1995) & Drinking water & Deionized water & NR & $<10 \mathrm{ppm}$ \\
\hline Niu et al. (2008) & Drinking water & Double distilled water & NR & NR \\
\hline Niu et al. (2009) & Drinking water & Double distilled water & NR & NR \\
\hline Niu et al. (2014) & Drinking water & Double distilled water & NR & NR \\
\hline Paul et al. (1998) & Gavage & Tap water & NR & NR \\
\hline Pereira et al. (2011) & Drinking water & Tap water (0.7 ppm F) & $0.7 \mathrm{ppm}$ & NR \\
\hline Raghu et al. (2013) & Drinking water & Tap water $(0.5$ ppm F) & $0.5 \mathrm{ppm}$ & NR \\
\hline $\begin{array}{l}\text { Reddy and Karnati } \\
\text { (2015) }\end{array}$ & Intraperitoneal & NR & NR & NR \\
\hline $\begin{array}{l}\text { Rumiantsev et al. } \\
\text { (1988) }\end{array}$ & Gavage & NR & NR & NR \\
\hline Sarkozi et al. (2015) & Gavage & Distilled water & NR & NR \\
\hline
\end{tabular}


Systematic Literature Review on the Effects of Fluoride on Learning and Memory in Animal Studies

\begin{tabular}{lllcc}
\hline \multicolumn{1}{c}{ Reference } & $\begin{array}{c}\text { Route of } \\
\text { Administration }\end{array}$ & \multicolumn{1}{c}{ Vehicle Control } & $\begin{array}{c}\text { Background } \\
\text { Fluoride in } \\
\text { Drinking Water }\end{array}$ & $\begin{array}{c}\text { Background } \\
\text { Fluoride in Feed }\end{array}$ \\
\hline Shen et al. (2004) & Drinking water & Tap water & $\mathrm{NR}$ & $\mathrm{NR}$ \\
Sun et al. (2008) & Drinking water & Tap water & $\mathrm{NR}$ & $\mathrm{NR}$ \\
Wang et al. (2004) & Drinking water & Drinking water $(<0.6 \mathrm{ppm} \mathrm{F)}$ & $0.6 \mathrm{ppm}$ & $25.57 \mathrm{mg} / \mathrm{kg}$ \\
Wang et al. (2006) & Drinking water & Free water & $\mathrm{NR}$ & $\mathrm{NR}$ \\
Wei et al. (2014) & Drinking water & Tap water (<1 ppm NaF) & $0.45 \mathrm{ppm}$ & $<5 \mathrm{mg} / \mathrm{kg}$ \\
Whitford et al. (2009) & Drinking water & Distilled drinking water & $\mathrm{NR}$ & $<0.01 \mathrm{mg} / \mathrm{kg}$-d \\
Wu et al. (2006) & Drinking water & Distilled water & $\mathrm{NR}$ & $\mathrm{NR}$ \\
Wu et al. (2008) & Drinking water & Tap water (0.6 ppm F) & $0.6 \mathrm{ppm}$ & $\mathrm{NR}$ \\
Xu et al. (2001) & Drinking water & Distilled fluoride-free water & $\mathrm{NR}$ & $\mathrm{NR}$ \\
Zhang et al. (2013) & Gavage & Drinking water $(<0.07$ ppm & $0.07 \mathrm{ppm}$ & $\mathrm{NR}$ \\
Zhang et al. (1999) & Drinking water & Distilled water & $\mathrm{NR}$ & $\mathrm{NR}$ \\
Zhang et al. (2001) & Drinking water & Deionized water & $\mathrm{NR}$ & $\mathrm{NR}$ \\
Zhang et al. (2009) & Drinking water & Fluoride-free water & $\mathrm{NR}$ & Fluoride-free \\
Zhu et al. (2012) & Drinking water & Distilled water & $\mathrm{NR}$ & $\mathrm{NR}$ \\
\hline
\end{tabular}




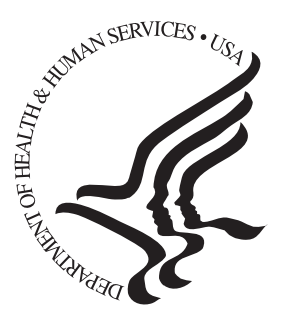

\title{
National Toxicology Program
}

\author{
NTP Central Data Management, MD K2-05
}

National Institute of Environmental Health Sciences

P.O. Box 12233

Research Triangle Park, NC 27709

http://ntp.niehs.nih.gov 